José Rodolfo Chreim

\title{
Development of a propeller Lifting-Line tool for analysis and design
}

São Paulo 

José Rodolfo Chreim

\title{
Development of a propeller Lifting-Line tool for analysis and design
}

\author{
Revised Version \\ (Original version is found at the unit that hosts this Graduate Program) \\ Master Dissertation submitted to the Escola \\ Politécnica of the University of São Paulo in \\ partial fulfillment of the requirements for the \\ degree of Master of Science \\ Concentration area: Mechanical Engineering \\ of Energy and Fluids \\ Advisors: \\ Prof. Ph.D. Marcos de Mattos Pimenta \\ Prof. Ph.D. Gustavo Roque da Silva Assi
}

São Paulo

2020 
Autorizo a reprodução e divulgação total ou parcial deste trabalho, por qualquer meio convencional ou eletrônico, para fins de estudo e pesquisa, desde que citada a fonte.

Este exemplar foi revisado e corrigido em relação à versão original, sob responsabilidade única do autor e com a anuência de seu orientador.

São Paulo, de de

Assinatura do autor:

Assinatura do orientador:

\section{Catalogação-na-publicação}

Chreim, José Rodolfo

Development of a propeller Lifting-Line tool for analysis and design / J. R.

Chreim -- versão corr. -- São Paulo, 2020.

$133 \mathrm{p}$.

Dissertação (Mestrado) - Escola Politécnica da Universidade de São Paulo. Departamento de Engenharia Mecânica.

1.Aerodynamics 2 .Hydrodynamics 3.Lifting-Line Theory 4.Wings 5.Propellers I.Universidade de São Paulo. Escola Politécnica. Departamento de Engenharia Mecânica II.t. 


\section{Acknowledgements}

Thank God for not letting me give up during the most desperate and hardest times. I have found resilience and patience through Your grace.

To my family for supporting me during times of anxiety and lack of time. Although they could not always understand, being respectful when I needed was more than sufficient at those times. It finally came to the right end!

To Joao Dantas for the seemingly endless, although fruitful, discussions. I could certainly learn much from you, but I insist that you should make barbecues with small chicken drumsticks.

To Fillipe and Gustavo Goes for helping me making the many CFD simulations go fast. I just hate those black screens.

To Eduardo Katsuno and Felipe Castro for the fun time we have had at the lab, the many meals together, and the conferences. Paga nóis

To Bruno Cheng, A.K.A. Bruxeng, for his wizardry on our side projects. To Andre for being in a tight corner in my place during the PRADS conference. To all my other fellows at the institute.

To Ana Paula for supporting me during this period in life. It finally ended, and we grew so much together during this time in life.

To Professor Marcos Ortega, for his invaluable help to the core of my work and the long e-mails we have exchanged.

To Professor Marcos Pimenta for his willingness to supervise me, his belief, and the freedom he gave me throughout the thesis. I think we have had a fun, productive time, professor!

To Professor Gustavo Assi for his kindness, attention and support. Thank you also for all the time provided to help me with the various aspects other than the thesis, and for the small talks.

To all my friends that supported me and were interested in my research, but had no idea about what I was doing. I have caught myself on this situation many times too, so no sweat. 



\section{Resumo}

O desenvolvimento de propulsores marítimos é fundamental no projeto de embarcações, visto que a determinação embarcação-propulsor apropriada possibilita maximizar a relação empuxo-torque, diminuir emissões de gases estufa ou ainda minimizar vibrações. O projeto de propulsores é complexo e requer conhecimento de diversas disciplinas em conjunto ao uso de ferramentas de engenharia. Em se tratando da hidrodinâmica de propulsores, as ferramentas existentes podem ser classificadas de acordo com o custo-fidelidade, e ora é mais interessante rapidez em detrimento à precisão - como na etapa preliminar -, ora é mais adequado precisão às custas de tempo - como na etapa de detalhamento. $\mathrm{O}$ objetivo deste trabalho é apresentar uma moderna formulação da Linha de Sustentação para análise de propulsores: dado um propulsor, a formulação rapidamente obtém curvas de empuxo e torque em água aberta em regiões próximas à de operação ótima. A vantagem está na rapidez em se avaliar a influência das características do propulsor nestes parâmetros. Seu desenvolvimento se inicia a partir da Linha de Sustentação para asas: adaptações são feitas para incorporação da viscosidade na sustentação e no arrasto e para asas com enflexamento e diedro, funcionando adequadamente para regimes de vôo até o início do estol. A formulação de asas é adaptada para propulsores moderadamente carregados, transportando as propriedades de inclusão de viscosidade nos cálculos das forças e momentos e de propulsores com distribuições de caimento e assimetria de contorno. Ambas as formulações são verificadas e validadas através de procedimentos recomendados por sociedades creditadas; a verificação se limita ao estudo de convergência de malha, e a validação é feita pela comparação dos resultados numéricos com dados experimentais disponíveis na literatura. Da verificação resultam taxas de convergência satisfatórias para pelo menos uma das discretizações propostas, indicando possibilidade de simulação das asas e propulsores propostos; a validação demonstra boa aderência entre os resultados numéricos e experimentais para faixas de operação dentro da validade da teoria de fólio fino (asas) e próximas do coeficiente de avanço de projeto (propulsores), indicando a capacidade de simulação, as limitações associadas aos regimes de operação e a influência da viscosidade nas forças e momentos. As formulações atendem as expectativas e especial atenção é dada à de propulsores, pois foi desenvolvida para análise hidrodinâmica, algo não típico de métodos de linha de sustentação. A incorporação de viscosidade e a simulação de propulsores com assimetria de contorno e caimento - sem a necessidade de métodos auxiliares - expandem sua aplicação em relação à teoria original, assim criando uma ferramenta de baixo custo e boa fidelidade.

Palavras-Chave: Aerodinâmica; Hidrodinâmica; Teoria da Linha de Sustentação; Asas; Propulsores. 



\section{Abstract}

The development of marine propellers is fundamental in ship design, as the appropriate hull-propeller combination may provide thrust-torque ratio optimization, greenhouse gas emissions reductions, and vibration minimization. Propeller design is already a complex task that requires the knowledge of many areas in addition to the use of diverse engineering tools. Particularly in hydrodynamics, these tools can be classified according to a trade-off between cost and fidelity: while it is sometimes more interesting to have a not-as-accurate, but a fast tool - as during early-stage design -, other times it is more appropriate a more precise, however costly, tool - as during refinement stage. This work presents a modern formulation of the Lifting-Line Theory for preliminary hydrodynamic propeller analysis, i.e., given a propeller geometry, the formulation is capable of obtaining the thrust and torque for a given advance velocity. Its importance lies in the fast configuration assessment and on the possibility of rapidly changing geometry characteristics. The development of the formulation starts from the wing lifting-line, in which adaptations are made to incorporate the influence of viscosity on lift and drag and to make it suitable for wings with dihedral and sweep at flow regimes until stall onset. Once established, the wing lifting-line is then adapted to the moderately loaded propeller problem, i.e., those whose operation sheds almost constant-pitch helical wakes; therefore, given the hypothesis, the adaptation conveys the properties of the influence of viscosity on thrust and torque, and the suitability to simulate propellers with rake and skew distributions. Both formulations are verified and validated according to procedures recommended by credited societies; verification is limited to mesh convergence analysis, while validation is done through comparison between numerical and experimental data, obtained from tests available on the literature. For both wings and propellers, verification presented satisfactory convergence rates for at least one of the proposed discretization schemes, indicating the possibility of simulating wings with dihedral and sweep and propellers with rake and skew; validation presents adequate adherence between numerical and experimental results for regions in which the thin-foil-theory is valid (wings) and near design advance ratio (propellers), results that indicate the capability of the methods for simulating wings and propellers with the proposed geometries, the limitations associated with the flow regimes, and the influence of the viscosity on forces and moments. In summary, the proposed formulations meet the expectations and special attention is given to the propeller one as it is oriented towards hydrodynamic analysis, typically not possible for such methods. Additionally, the inclusion of viscosity and the possibility of simulating raked and skewed propellers without further corrections broadens the range of application, thus creating a relatively low-cost-good-fidelity tool.

Keywords: Aerodynamics; Hydrodynamics; Lifting-Line Theory; Wings; Propellers. 



\section{List of Abbreviations and Acronyms and Abbreviations}

AoA Angle of Attack

AR Aspect Ratio

ASME the American Society of Mechanical Engineers

BC Boundary Condition

BEM Boundary Element Method

BEMt Blade Element Momentum theory

BV Bound Vortex

CCW Counter Clockwise

CFD Computational Fluid Dynamics

CP Control Point

CW Clockwise

DARPA Defense Advance Research Projects Agency

DES Detached Eddy Simulations

DNS Direct Numerical Simulations

DTMB David Taylor Model Basin

EAR Expanded Area Ration

EEDI Energy Efficiency Design Index

FD Finite Difference

GCI Grid Convergence Index

HHSV Helical Horseshoe vortex

HSV Horseshoe Vortex

ImHHSV Image Helical Horseshoe vortex 
IMO International Maritime Organization

IPT Institute for Technological Research

ITTC International Towing Tank Conference

KCA Kings College Admiralty

KJT Kutta-Joukowski theorem

LES Large Eddy Simulation

LHS Latin Hypercube Sampling

LLT Lifting-Line Theory

LSM Least Square Method

LST Lifting-Surface Theory

LVD Loop-Vortex Density

MARIN Maritime Institute Netherlands

MC Monte Carlo

MMS Method of Manufactured Solutions

NSRDC Naval Ship Research and Development Center

PBC Pistolesi Boundary Condition

PLL Propeller Lifting-Line

PPTC Potsdam Propeller Test Case

RANS Reynolds Averaged Navier-Stokes

SHSV Straight Horseshoe Vortex

SS Systematic Series

SST Shear Stress Transport

SVA Schiffbau-Versuchsanstalt Potsdam Model Basin

SV Starting Vortex

TE Trailing Edge

TFT Thin Foil Theory 
TV Trailing Vortex

VV Verification and Validation

WLL Wing Lifting-Line 



\section{List of symbols}

\begin{tabular}{|c|c|}
\hline$b$ & Wing span \\
\hline$C_{c}$ & Foil tangential force coefficient \\
\hline$C_{D}$ & Wing drag coefficient \\
\hline$C_{L}$ & Wing lift coefficient \\
\hline$C_{n}$ & Foil normal force coefficient \\
\hline$c_{0}$ & Chord at the root of the wing \\
\hline$C_{T}$ & Propeller thrust loading coefficient \\
\hline$D$ & Total drag \\
\hline$\frac{\partial C_{n}}{\partial \alpha}$ & Airfoil section normal force slope \\
\hline$\vec{F}_{p}$ & Potential aerodynamic force vector \\
\hline$\vec{F}_{v}$ & Viscous aerodynamic force vector \\
\hline$K_{T}$ & Propeller thrust coefficient \\
\hline$K_{Q}$ & Propeller torque coefficient \\
\hline$N$ & Number of horseshoe vortices \\
\hline$\vec{r}_{i j}$ & Vector from point $\mathrm{i}$ to point $\mathrm{j}$ \\
\hline$L$ & Total Lift \\
\hline$\hat{u}_{n}$ & Local unit vector normal foil section \\
\hline$R e_{\infty}$ & Free-stream Reynolds number \\
\hline$\vec{V}_{\infty}$ & Free-stream advance or cruise velocity vector \\
\hline$\vec{V}_{j}$ & Total velocity vector over a $\mathrm{j}^{\text {th }}$ bound vortex \\
\hline$\vec{V}_{S H V}$ & Induced velocity by a single horseshoe vortex \\
\hline$\alpha$ & Geometric angle of attack \\
\hline$\alpha_{e f f}$ & Effective angle of attack \\
\hline
\end{tabular}




$\begin{array}{ll}\alpha_{i} & \text { Induced angle of attack } \\ \alpha_{L 0} & \text { Zero-lift angle of attack } \\ \Delta & \text { Absolute difference } \\ \delta \vec{l} & \text { Length vector along the bound vortex segment } \\ \gamma & \text { Wing dihedral angle. } \\ \vec{\Gamma} & \text { Circulation vector } \\ \Lambda & \text { Wing sweep angle } \\ \rho_{\infty} & \text { Free-stream density } \\ \theta & \text { Wing or blade geometric twist angle }\end{array}$




\section{Contents}

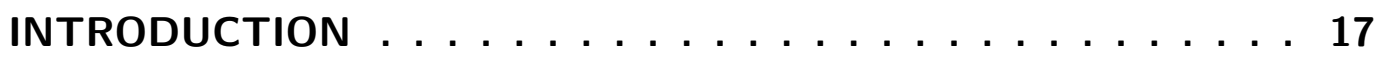

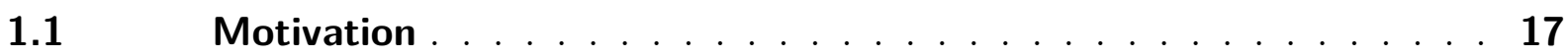

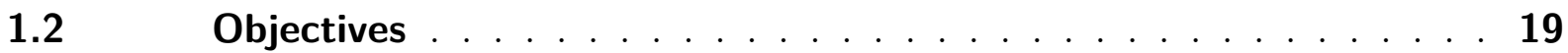

1.3 Review of the Literature . . . . . . . . . . . . . . . . 20

1.3.1 Wing Lifting-Line Theory . . . . . . . . . . . . . . . . . 23

1.3.2 Propeller Lifting-Line Theory . . . . . . . . . . . . . . . . . . 28

2 MATHEMATICAL AND PHYSICAL MODELS . . . . . . . 33

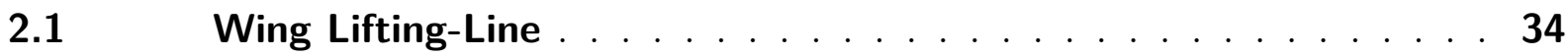

$2.1 .1 \quad$ Linear scheme . . . . . . . . . . . . . . . . . . . . . 36

2.1.2 Nonlinear scheme . . . . . . . . . . . . . . . . . . . . 37

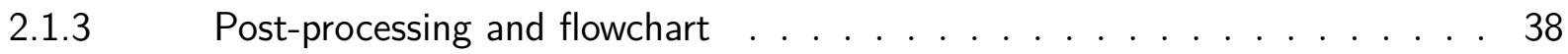

$2.2 \quad$ Propeller Lifting-Line $\ldots \ldots \ldots \ldots$

$2.2 .1 \quad$ Linear scheme . . . . . . . . . . . . . . . . . . . . 45

2.2.2 Nonlinear scheme . . . . . . . . . . . . . . . . . . . . . . 46

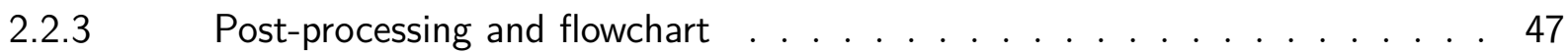

$2.3 \quad$ Source of $2-\mathrm{D}$ data on the lifting-line . . . . . . . . . . . . 48

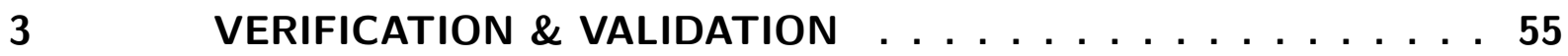

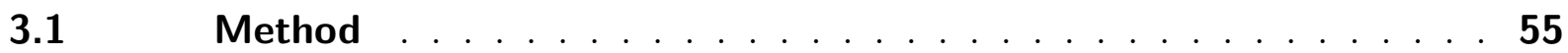

$3.2 \quad$ Code Verification and Solution Verification . . . . . . . . 56

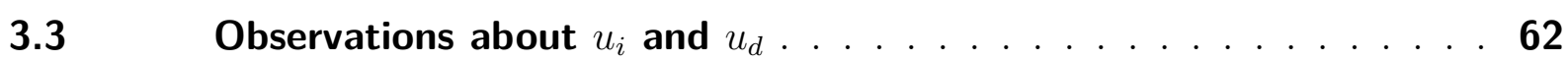

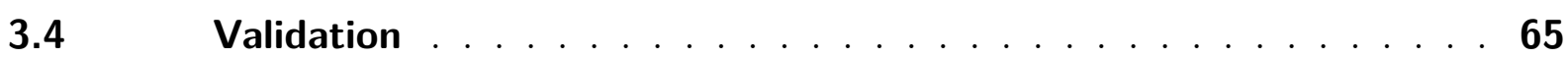

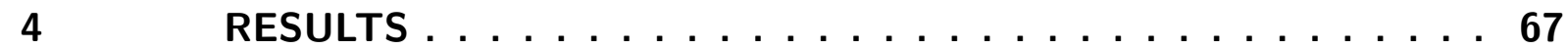

$4.1 \quad$ Wing Lifting-Line . . . . . . . . . . . . . . . . . . 68

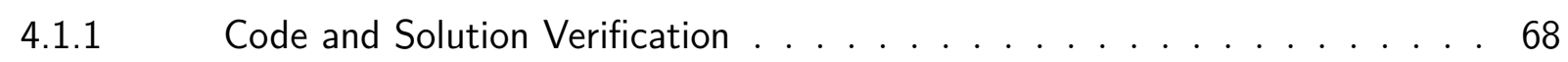

4.1.1.1 Elliptically-Loaded Wings Under Potential Flow . . . . . . . . . . . . . 68

4.1.1.2 Rectangular Wing Under Viscous Flow . . . . . . . . . . . . . . . . 73

4.1.1.3 45-deg Sweptback Wing Under Potential Flow . . . . . . . . . . . . . 74

4.1.1.4 10-deg Dihedral Wing Under Potential Flow . . . . . . . . . . . . 75

4.1.1.5 Conclusions about Code and Solution Verifications . . . . . . . . . . 75

$4.1 .2 \quad$ Validation . . . . . . . . . . . . . . . . . 76

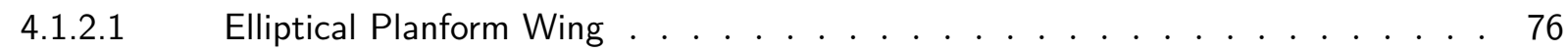

$4.1 .2 .2 \quad$ Crescent Wing . . . . . . . . . . . . . . . . . . 78

4.1.2.3 Rectangular Wing . . . . . . . . . . . . . . . . . . . . . 79 
4.1.2.4 45-deg Sweptback Wing . . . . . . . . . . . . . . . . . . 81

4.1.2.5 Validation Tables and Conclusions about Validation . . . . . . . . . . . . 82

$4.2 \quad$ Propeller Lifting-line . . . . . . . . . . . . . . . . . . . . 85

4.2.1 Code and Solution Verification . . . . . . . . . . . . . . . . . . . . 85

4.2.1.1 Wake model . . . . . . . . . . . . . . . . . 85

4.2.1.2 DTMB 4119 Propeller . . . . . . . . . . . . . . . . . . . . . . 91

4.2.1.3 Conclusions about Code and Solution Verifications . . . . . . . . . . . . . 92

$4.2 .2 \quad$ Validation . . . . . . . . . . . . . . . . . . . . 94

4.2.2.1 DTMB 4119 Propeller . . . . . . . . . . . . . . . . . . . . . . . . . 94

4.2.2.2 DTMB 4133 Propeller . . . . . . . . . . . . . . . . . . . . . . 96

4.2.2.3 DTMB 4381 Propeller . . . . . . . . . . . . . . . . . . . . . . . . . . . 99

4.2.2.4 H1 Propeller . . . . . . . . . . . . . . . . . . . . . 102

4.2.2.5 MOD5 Propeller . . . . . . . . . . . . . . . . 105

4.2.2.6 PPTC Propeller . . . . . . . . . . . . . . . . 107

4.2.2.7 Validation Tables and Conclusions about Validation . . . . . . . . . . . . . 109

5 CONCLUSION AND FUTURE WORK . . . . . . . . . 113

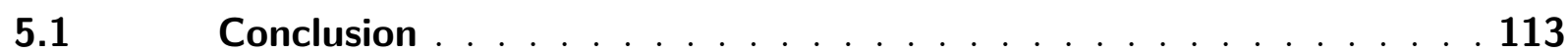

$5.2 \quad$ Future Work . . . . . . . . . . . . . . . . . . . . . 114

5.2.1 PLL-CFD Coupled Methods . . . . . . . . . . . . . . . . . . . 114

5.2.2 Hydroelastic Propellers . . . . . . . . . . . . . . . . . . . . . . . 114

5.2.3 General Changes in the Propeller Formulation . . . . . . . . . . . . . . . . 114

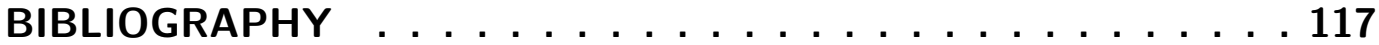

$\begin{array}{ll}\text { APPENDIX } & 123\end{array}$

APPENDIX A - NECESSARY MATHEMATICAL AND PHYSICAL DERIVATIONS . . . . . . . . . . . . . . 125

A.1 Reasoning for keeping part of the HSV over the wing/blade planforms125

A.2 Velocity induced by a straight vortex segment . . . . . . . . . . 127

A.3 Resultant section potential force . . . . . . . . . . . . . . . 129

A.4 Representation of the resultant force vector in the wing coordinate system . . . . . . . . . . . . . . . . . . . . . . 130 


\section{Introduction}

\subsection{Motivation}

In recent years, the knowledge in the emission estimates for greenhouse gases from ships made the International Maritime Organization (IMO) adopt an Energy Efficiency Design Index (EEDI) for ship design, to lower costs and emissions while increasing efficiency. An important part of ship design is the design of propellers with optimum performance, a multidisciplinary complex process that demands knowledge and efficient use of several tools, always seeking for the best a compromise among thrust, torque, cavitation, structural resistance, vibration, noise, and manufacturing cost; according to recommendations of the $27^{\text {th }}$ International Towing Tank Conference (ITTC) Propulsion Committee (BERTSCHNEIDER et al., 2014; ITTC, 2017), for instance, effort should be directed towards the assessment of the potential impact of new technological developments, including new types of propulsion systems (hybrid propulsion), azimuth thrusters, cycloid propulsors, and flexible blade propellers.

In terms of hydrodynamic propeller design, several analytic and numerical tools currently exist, each one best suited to a specific design stage; examples of these tools are the Systematic Series (SS), Blade Element Momentum theory (BEMt), Lifting-Line Theory (LLT) methods, Lifting-Surface Theory (LST) methods, Boundary Element Method (BEM), and the commonly (and inappropriately) known as Computational Fluid Dynamics (CFD). With different characteristics from one another, they can be used interchangeably during the design cycle.

Regarding the SS approach, since its first use in 1850, many standard propellers series have been tested in laboratories around the world to provide a database to help the designer understand the factors of influence on propeller performance and cavitation inception; from the database, design diagrams have been created aiming assistance on the selection of the most appropriate parameters of a propeller to suit the needs of a specific ship. Some of the important series for which this approach has been done is the Wageningen B-screw, the Japanese AU, the Gawn, the KCA, the Lindgen (Ma), and the Newton-Rader (CARLTON, 2019). Designing a propeller through SS approach is considerably fast, simple, and still largely used, but it is confined to the experimental ranges and parameters by which the propellers have been subjected.

The BEMt approach is based on dividing the propeller blade up into a large number of elementary strips, which can then be regarded as foils subject to a resultant incident velocity - considered to comprise an axial velocity together with a rotational velocity. 
The difference between the hydrodynamic pitch angle and the blade pitch angle at each section gives rise to an incidence or effective Angle of Attack (AoA). Each section, thus, experiences lift and drag forces from the combination of the incidence and the section zero lift AoAs. This theoretical model allows the thrust and torque to be calculated given appropriate values of normal and axial section coefficients are known, a drawback in the model as they cannot be readily calculated. Additional limitations, such as flow and geometric, also exist, but the BEMt represented a great advance in propeller design, and it contained basic ideas upon which modern theories are founded (CARLTON, 2019).

LLT methods model each blade by a series of Horseshoe Vortex (HSV) either superimposed one another (classic approach), or placed alongside (vortex-step or vortexlattice approach), and each section is treated similarly to the BEM approach. They can be used for either propeller preliminary design or analysis and present an intermediate compromise between cost and accuracy. Used as an analysis tool, they predict important features such as thrust and torque coefficients, load distributions, thrust and torque loads coefficients, and induced velocities along with the blades (FLOOD, 2009). As a design tool, coupled to LST correction factors (MORGAN; SILOVIC; DENNY, 1968), for a desired circulation distribution $\Gamma$ (or a hydrodynamic force distribution), chord, pitch angle, and maxima section thickness and curvature distributions are determined, while cavitation inception (KERWIN; HADLER, 2010) is minimized; current research has shown that coupling LLT with CFD reduce simulations CPU time while it still provides accurate results(BERTSCHNEIDER et al., 2014). Examples of theses couplings are hull-propeller assemblies, ducted propellers, contra-rotating propellers, and even assemblies of wind farms. The classic model, however, presents limitations in terms of propeller geometry, flow regime, and propeller loading condition.

LST methods are natural extensions of the LLT, having KERWIN 1973 as one of their main contributors; the propeller and its wake are modeled as a series of HSV that span both in the spanwise and chordwise directions that satisfy the Kutta and no-normal flux conditions and whose strengths are determined by the induced velocities, from the Biot-Savart's law. They are used to both calculate the hydrodynamic forces and moments and to (iteratively) adapt the wake form (BERTRAM, 2012). By using the LST, more complex geometries can be analyzed and more accurate results obtained, but with a price of higher computational cost due to the required discretization. This last trend makes these types of methods appropriate to an intermediate design/analysis stage.

BEMs, also known as Panel Methods, are potential-flow based formulations that discretize the actual (and not a reference) geometry into a series of elements populated by distributions of singularities, such as sources and doublets (or vortices). These unknown singularities are then solved with the aid of non-flux boundary conditions, so the fluid forces, velocity and pressure fields, and even cavitation inception regions are obtained 
(VAZ, 2005). Panel methods are currently the most used tool for propeller design, but despite their capabilities, the inclusion of viscous effects as well as the generation of appropriate "meshes" can be cumbersome in comparison to simpler methods such as the lifting-line.

Finally, CFD usually refers to numerical schemes in which the flow field is specified through a Eulerian formulation and the physical domain (thus governing equations) is discretized using finite differences, finite elements, or finite volumes. During the last decades, considerable advances in terms of grid generation, numerical models, and even solvers have been made, making these methods gradually gain acceptance from academia and industry to the particular point that important insights about the physics of the flow can be drawn; in the particular case of propellers, the influence of viscosity and the type and inception of cavitation, for instance. Some of the approaches to model the physics of the flows are the Reynolds Averaged Navier-Stokes (RANS) method, Large Eddy Simulation (LES) techniques, Detached Eddy Simulations (DES), and Direct Numerical Simulations (DNS) (CARLTON, 2019). Not all of such approaches, however, are suitable for modeling the flows around propellers, especially due to the high computational cost, which is the reason CFD is often appealing during design detail phases.

To develop an appropriate formulation for propeller analysis with further intention to help in the preliminary stages of the complex process of hull-propeller design, this work presents a tool capable of accurately simulating propellers with some degree of rake and skew and also considering the effects of viscosity on thrust and torque; as computation cost plays a major role in the choice of the method, and for preliminary stages, it must be relatively low, the tool is based on an extension of the classic Propeller Lifting-Line (PLL) that overcomes part of its limitations to attain the desired purposes.

\subsection{Objectives}

A wing formulation is first developed due to the vast literature about Wing LiftingLine (WLL) formulations adapted to account for the inclusion of viscosity on lift and drag and the simulation of wings with variable chord, sweep, and dihedral distributions. Then, this formulation is adapted to the propeller case, which ultimately inherits the wing formulation characteristics. The following activities have been part of the work:

- Development of a modern WLL method, in the sense that, contrary to the classic approach, it considers information about the airfoils on lift and drag and it also simulates wing geometries with arbitrary chord, sweep, and dihedral distributions;

- Verification and validation of the WLL, through comparison against theoretical results for the elliptical wing, assessment of the convergence behavior for several 
geometries, and comparison of $C_{L}$ and $C_{D}$ against experimental data;

- Adaptation of the WLL to the propeller case, obtaining a modern PLL method, which considers information about the hydrofoils on thrust and torque and it also simulates blade geometries with arbitrary chord, skew, and rake distributions;

- Verification and validation of the PLL, through comparison of the outputs of the wake model proposed with analytic results from the Wrench Formulas, assessment of the convergence behavior for several geometries, and comparison of $K_{T}$ and $K_{Q}$ against experimental data;

\subsection{Review of the Literature}

One of the first important applications in Potential flow theory was the study of lifting-surfaces such as wings and propellers. These problems can be reduced to the solution of a boundary value problem whose partial differential equation is simply the Laplace (continuity) Equation:

$$
\nabla^{2} \Phi=0
$$

subject to the following boundary conditions: (i) the disturbance induced by the lifting surface decays far from it

$$
\lim _{r \rightarrow \infty} \nabla \Phi=\vec{V}_{\infty}
$$

which is automatically fulfilled by singular solutions such as sources, doublets, and vortices, and (ii) the normal component of velocity on the solid boundaries of the lifting-surface must be zero. Thus, in a frame of reference attached to it:

$$
\nabla \Phi \cdot \hat{n}=0
$$

in which $\hat{n}$ is the unit vector normal to the surface of the body, pointing outwards. The analytic solution of such boundary value problem, for an arbitrary shape, is complicated due to the difficulty of specifying the boundary condition of equation (1.3) on complex geometry surfaces. To overcome this difficulty, some simplifying assumptions can be made, such as the small perturbation assumption and the first-order approximation of the Boundary Condition (BC), which leads to the following equation:

$$
\frac{\partial \Phi}{\partial z}(x, y, 0)=V_{\infty}\left(\frac{\partial \eta}{\partial x}-\alpha\right)
$$


with $\eta(x, y)$ the geometry solid surface, which can be divided into an upper $\eta_{u}$ and lower $\eta_{l}$ surface coordinates and also be expressed by thickness $t$ and camberline $f$ functions, such that:

$$
\begin{aligned}
f & =\frac{1}{2}\left(\eta_{u}+\eta_{l}\right) \\
t & =\frac{1}{2}\left(\eta_{u}-\eta_{l}\right)
\end{aligned}
$$

Specifying equation (1.4) for both surfaces, and given the fact that Laplace's equations and the associated boundary conditions (after the assumptions) are linear, the complete solution can be obtained by the superposition of three simpler problems: (i) symmetric geometry with nonzero thickness at zero AoA; (ii) zero-thickness, uncambered geometry at an AoA; and (iii) zero-thickness, cambered geometry at zero AoA. To illustrate the outcomes, consider the geometry represented in figure 1.

Figure 1 - Decomposition of the thick cambered foil at a given incidence angle problem into three simpler problems.

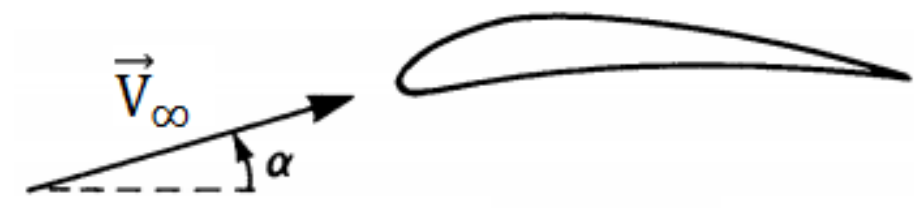

II
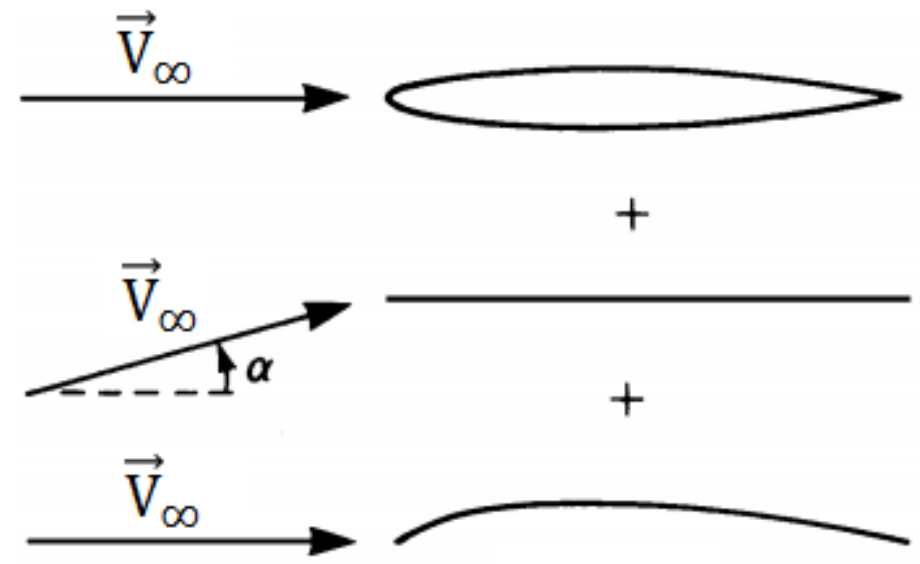

Source: KATZ; PLOTKIN, 2001 (Adapted).

The solution for the first problem can be obtained by distributing basic source/sink elements along the geometry, as those entities satisfy Laplace's equation and have vanishing induced velocities at infinity, in addition to generating symmetric flows (Figure 2); the obtained pressure and velocity distributions lead to the conclusion that there is no lift (or drag) produced due to thickness. 
Figure 2 - First of the three lifting surface problems: symmetric geometry section ( $y$ plane) with nonzero thickness at zero angle of attack.

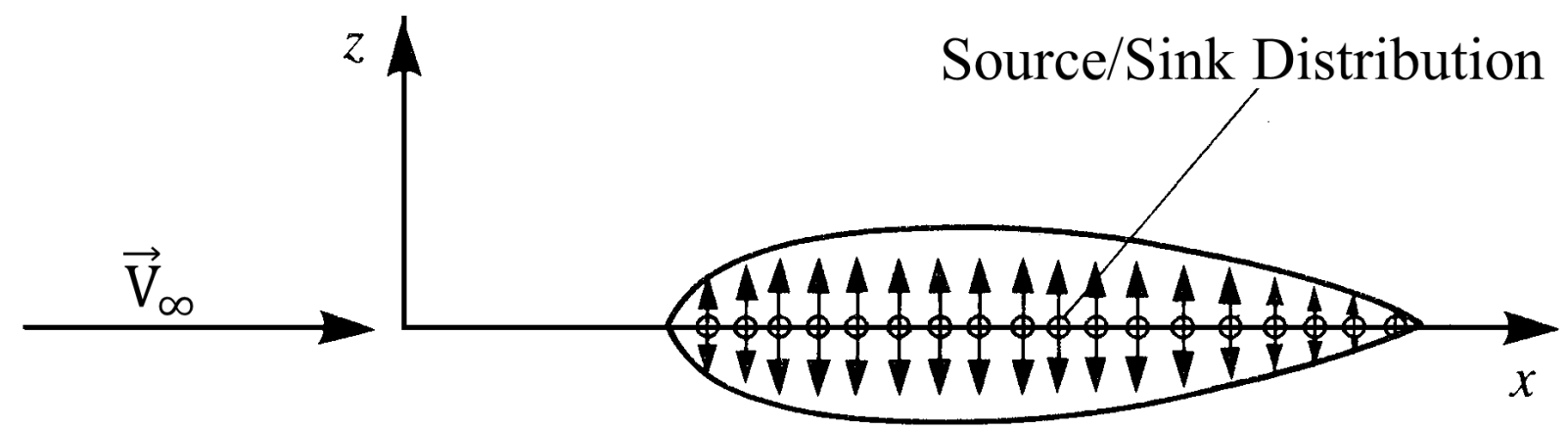

Source: KATZ; PLOTKIN, 2001 (Adapted).

Problems (ii) and (iii) - figure 3 - are antisymmetric to the flow plane and can be solved by vortex distributions $\gamma\left(\gamma_{y}\right.$ pointing in the $y$ direction and $\gamma_{x}$ in the $x$ direction) throughout the geometry; these elementary solutions to Laplace's equation (1.1) also automatically fulfill equation (1.2) and a unique distribution is obtained when the Kutta condition - which states that the flow must leave sharp trailing edges smoothly and with finite velocity or, equivalently, zero pressure difference - is satisfied. Thus, the lifting problem is reduced to finding combinations that meet the zero normal flow BC throughout the solid surfaces - equation (1.3). These vortex distributions, however, must also satisfy both Helmholtz's and Kelvin's Theorems (KATZ; PLOTKIN, 2001), which state that, in an incompressible inviscid flow with conservative body forces acting, (i) the strength of a vortex filament is constant along its length, (ii) a vortex filament (open curve) cannot start or end inside a fluid region, (iii) vortex elements remain the same with time, and (iv) the circulation strength of a fluid curve remains constant with time; thus, one of the possibilities is to model the vortex distributions as closed vortex rings with constant strength that must extend behind the geometry, into a wake whose starting vortex is located far downstream in a steady-state problem - figure 4.

Figure 3 - Second and third lifting surface problems: nonsymmetric geometry section (y plane) at nonzero angle of attack.

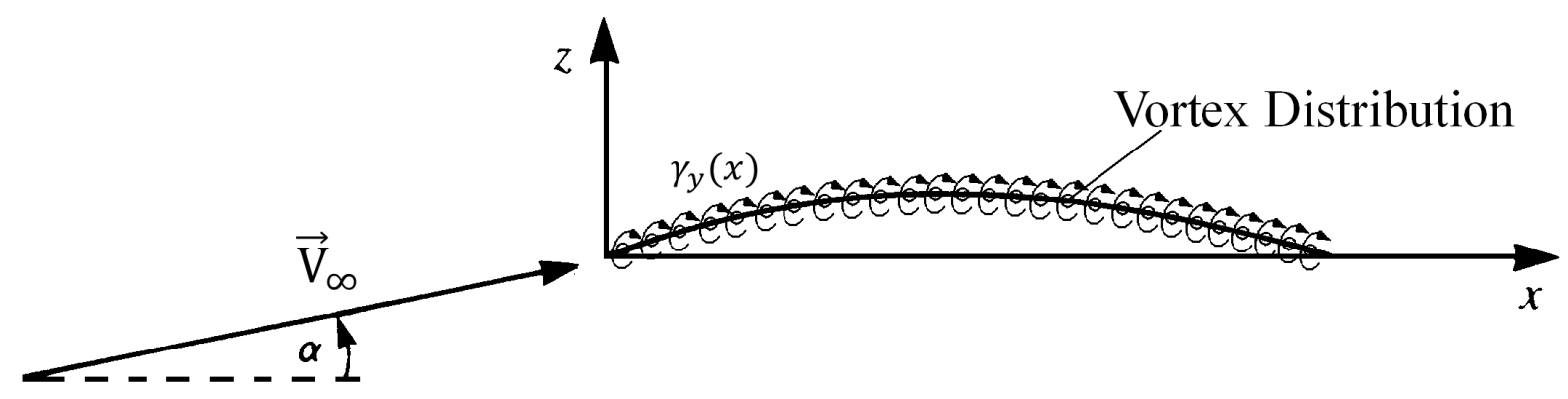

Source: Author (Adapted from KATZ; PLOTKIN, 2001). 
Figure 4 - lifting surface geometry modelled by constant strength $\gamma$ vortex-loops. $\gamma_{y}=\gamma_{x}$ due to the circulation theorems

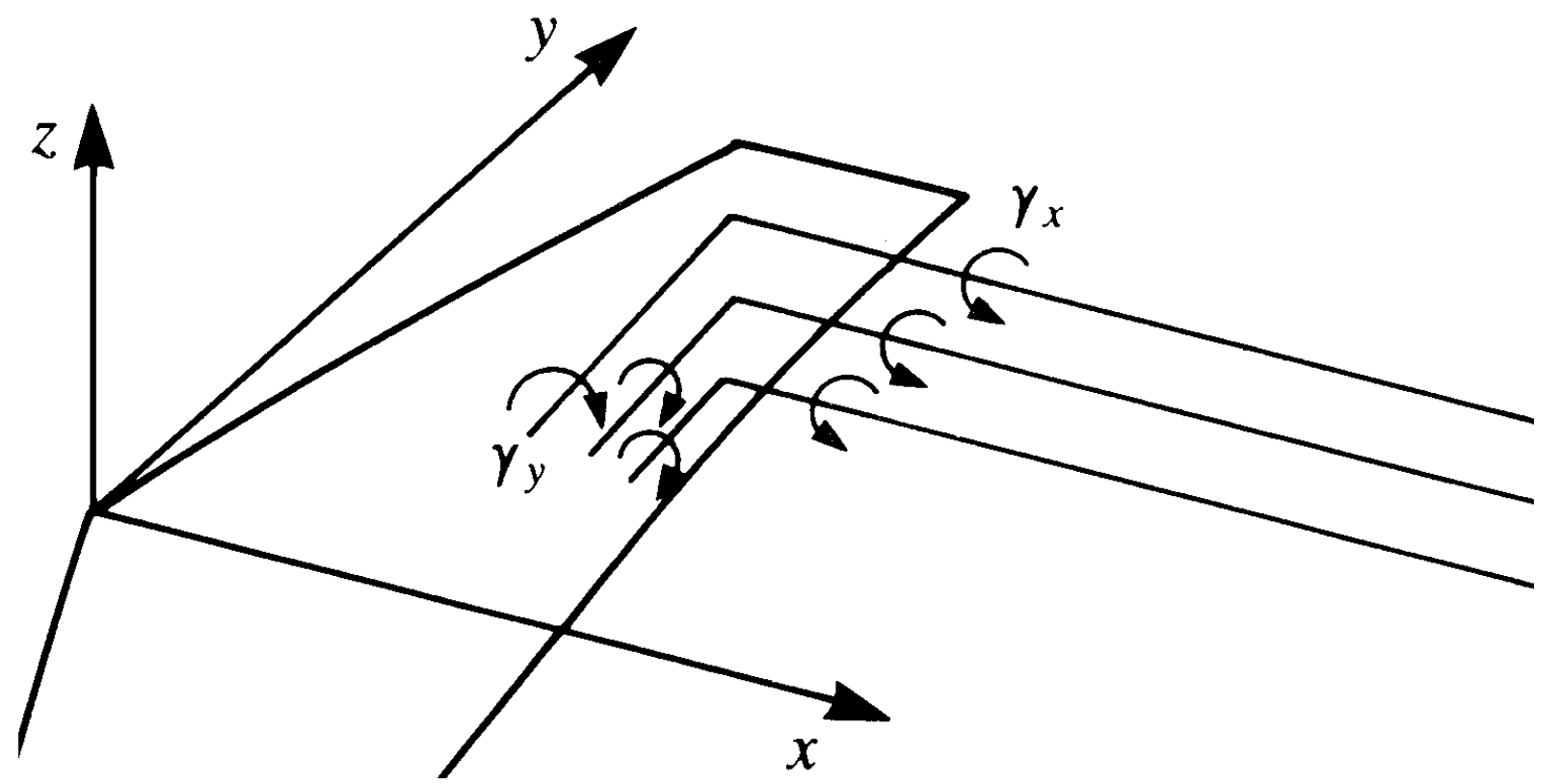

Source: KATZ; PLOTKIN, 2001 (Adapted).

\subsubsection{Wing Lifting-Line Theory}

The first and simplest model for predicting the aerodynamics of wings was developed independently by Frederick W. Lanchester in 1907 and by Ludwig Prandtl in 1918 - 1919 (JR, 2010; DRAGOS, 2004; KATZ; PLOTKIN, 2001), and it was based on the ideas and assumptions of potential flows: consider a section of the geometry depicted in figure 3 . The associated vortex distribution can be lumped into a single core vortex whose strength (or circulation) $\Gamma$ is the same as the integral of $\gamma_{y}$ along the chord and whose location is the center of pressure of the section; from the results of the Thin Foil Theory (TFT), for a flat plate, this is typically the quarter chord location - figure 5.

To satisfy both Helmholtz's and Kelvin's Theorems, the lumped vortex must be bent 90 degrees in both directions and close at infinity downstream closing the loop, as needed (Figure 6); while this geometry is commonly known as a horseshoe vortex, the lumped vortex is called Bound Vortex (BV), each segment bent backward is called Trailing Vortex (TV), and the one at infinity called Starting Vortex (SV) - figure 6. To have a variable circulation distribution, many HSVs are superimposed to one another, as in figure 7.

With this representation, Prandtl hypothesized that each segment of the wing would behave as the segment of an equivalent infinite wing with the same characteristics; hence, the wing segment would experience a force according to the Kutta-Joukowski theorem (KJT) (SAFFMAN, 1992) that depends on the overall velocity, which, in turn, 
Figure 5 - Vortex distribution on a flat plate and its equivalent lumped-vortex and associated lumped Pistolesi Boundary Condition (PBC) location.
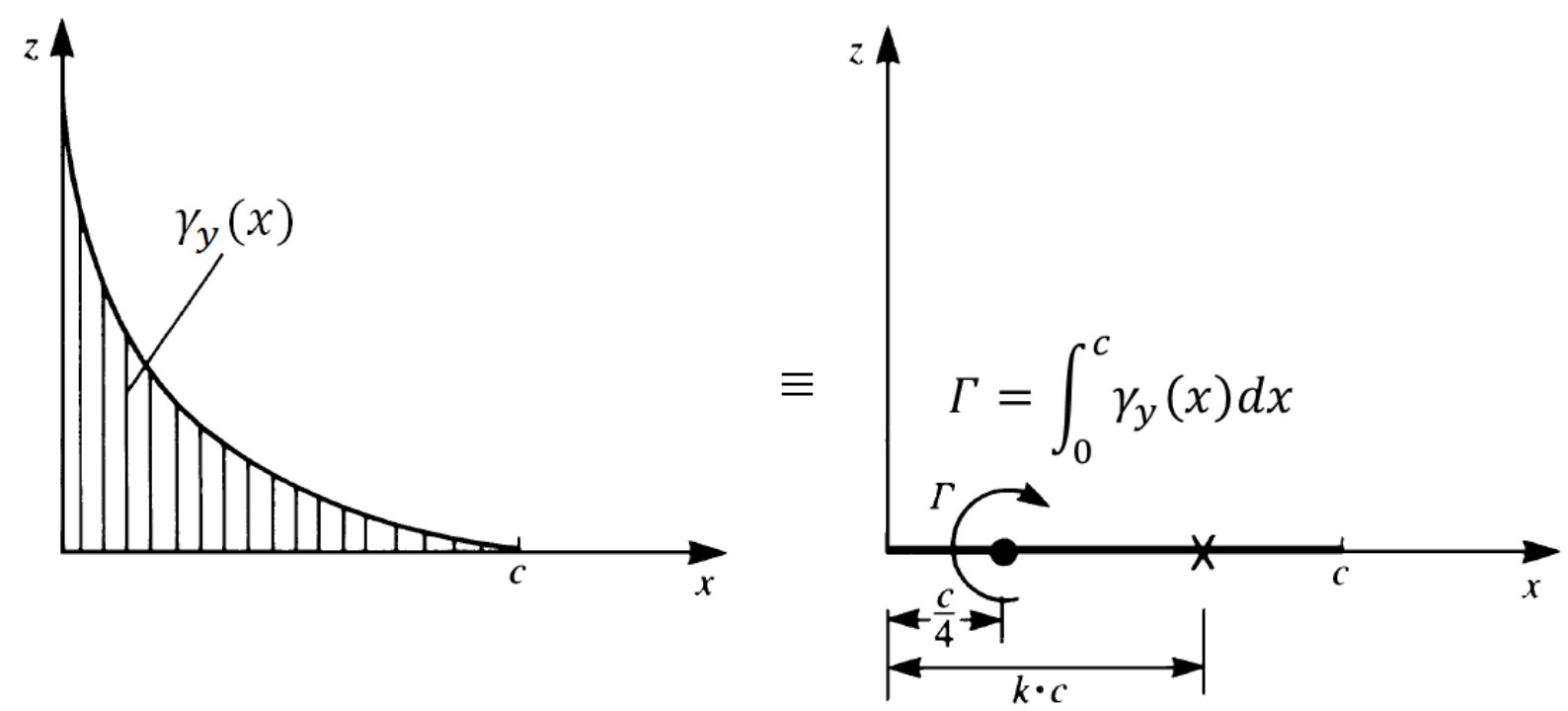

Source: KATZ; PLOTKIN, 2001 (Adapted).

Figure 6 - Example of HSV.

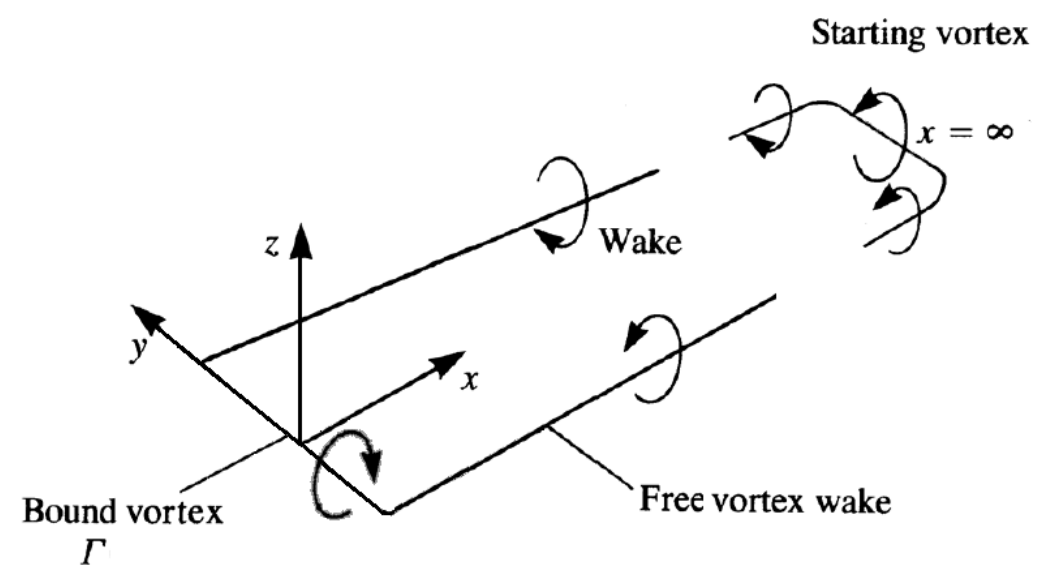

Source: Author (Adapted from KATZ; PLOTKIN, 2001).

depends on the wake-induced velocities - or downwash. The downwash is generated by both TVs and SV, although the latter has practically no influence on the wing due to their distance; additionally, on Prandtl's model, the BV induce no velocity over one another, as they must be straight parallel lines. The downwash is a consequence of the finiteness of the wing: it is a purely 3-D effect that changes the magnitude and direction of the local velocity $V$ by an induced angle of attack $\alpha_{i}$ that consequently changes the direction of the local aerodynamic force, so it is no longer perpendicular to $V_{\infty}$; thus, an induced drag drag component $D_{i}$ arises - figure 8 -, which can be viewed as a measure of the 'power' lost to generate the trailing vortices.

The induced velocities - consequently angles and aerodynamic forces - depend on 
Figure 7 - Prandtl's classic LLT model.

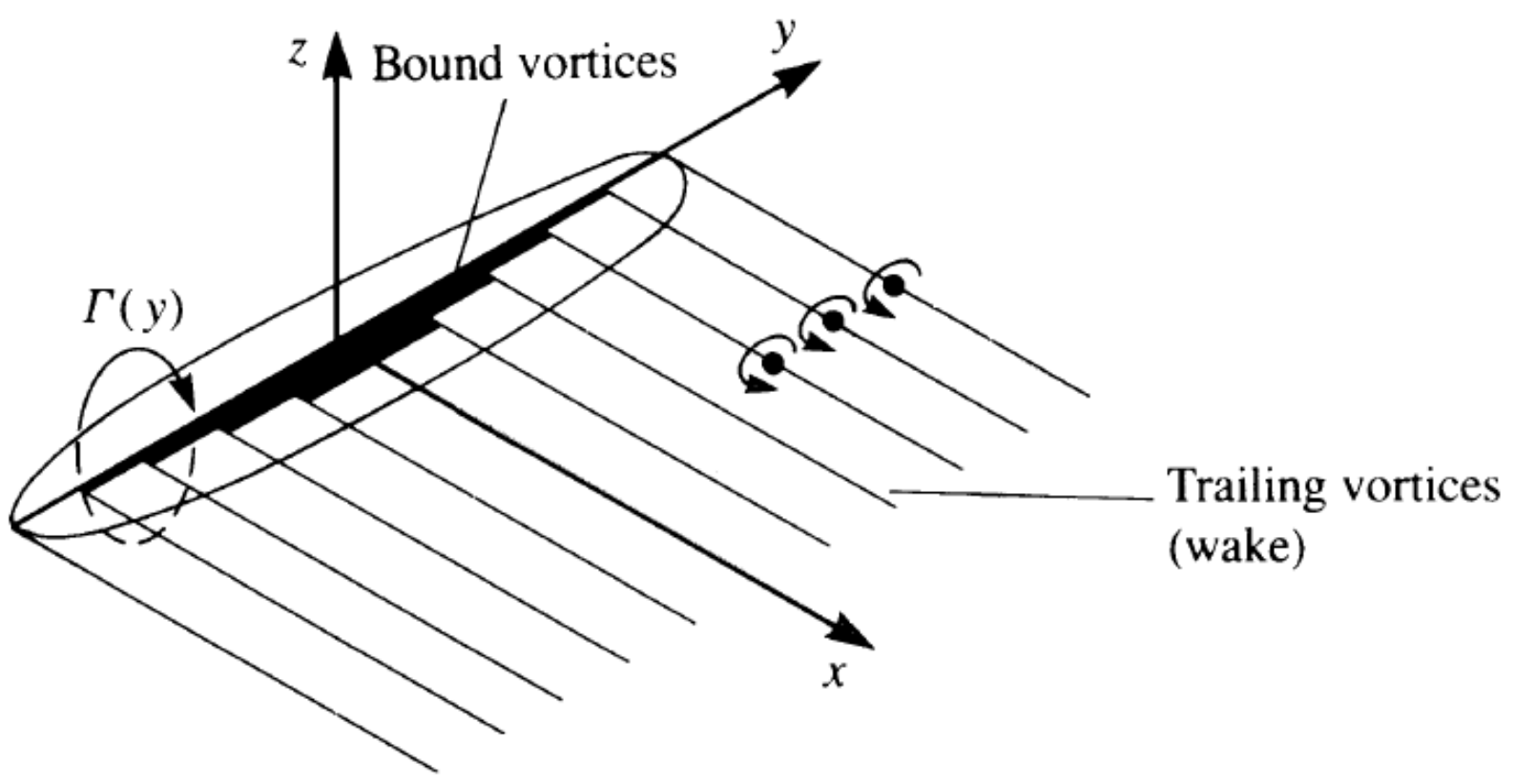

Source: KATZ; PLOTKIN, 2001 (Adapted).

Figure 8 - Wing section diagram of the LLT.

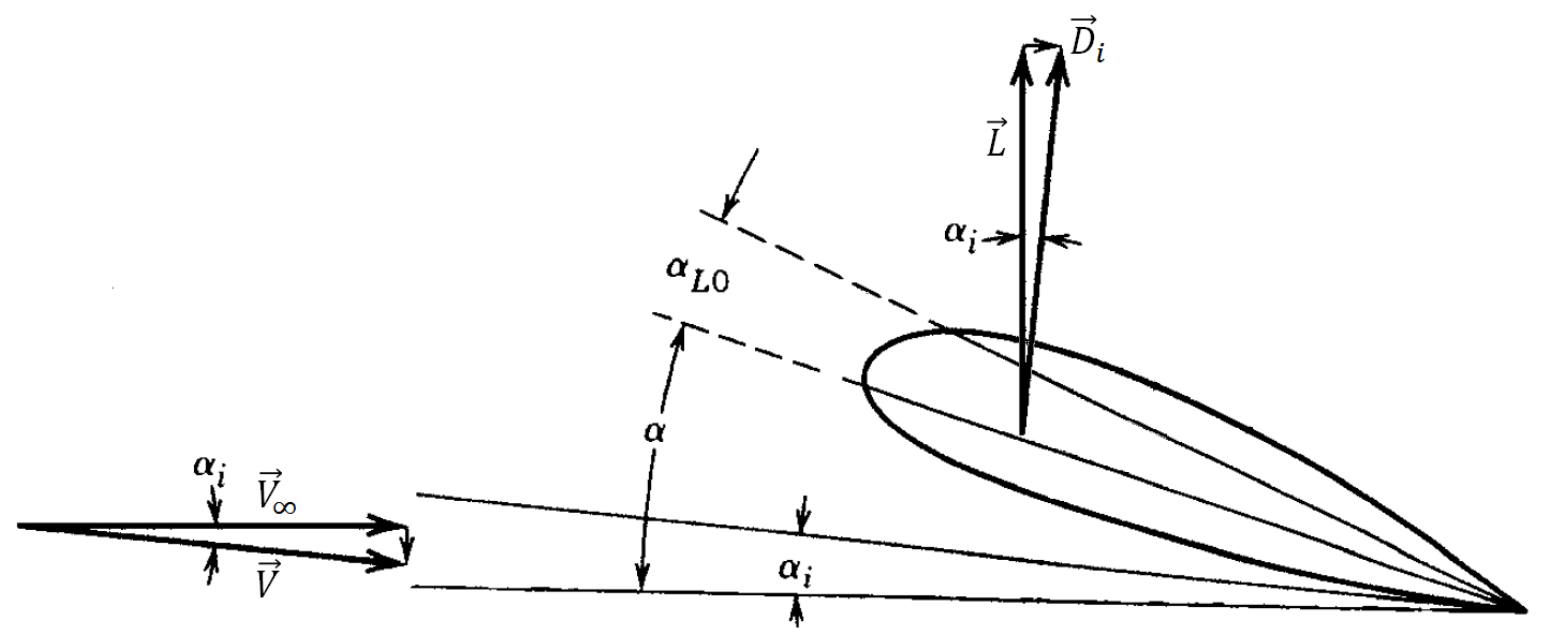

Source: KUETHE; CHOW (1997) (Adapted)

the knowledge of the circulation distribution (KATZ; PLOTKIN, 2001). Using the wing section-equivalence hypothesis, Prandtl derived a mathematical expression, known as the fundamental equation of the lifting-line theory, that related the several AoAs of figure 8; the expression is an integrodifferential equation for $\Gamma$. For some geometries, such as the flat-plate elliptical-planform wing, the equation has analytic solution, but for most of the cases it must be solved numerically; with the advent of numerical techniques, the LLT was used to obtain the circulation distribution of different geometries (although limited its assumptions), as in those cases the circulation distribution could be represented by a Fourier series and the integrodifferential equation transformed into a system of algebraic 
equations. The classic WLL brought insightful conclusions to the aerodynamics of wings that allowed advances in wing design, such as the maximum optimum lift-to-drag ratio characteristic of elliptically-loaded wings and the quantification of the effects of taper, geometric twist, and aspect ratio (KATZ; PLOTKIN, 2001); its limitations, however, made further extensions necessary as the advances in aerodynamic theory urged for more efficient geometries under higher flight speeds and real flow conditions (DRAGOS, 2004).

As the vortex distribution can be lumped, so the no-normal-flux BC: in 1937, Pistolesi demonstrated that, for a flat plate with lift curve slope of $2 \pi$, the BC was lumped to the $\frac{3 c}{4}$ location (PISTOLESI, 1937); using the PBC along with Prandtl's hypothesis, in 1947, Weissinger proposed one of the most important adaptations of the LLT (WEISSINGER, 1947): he assumed that PBC needed to be satisfied throughout the wing three-quarter-chord line (Figure 9). Even though his assumption fundamentally led to the same equations, the novel approach was capable of simulating wings having nonstraight quarter-chord lines as the velocity singularities at the BV were addressed, so he used it to numerically calculate a series of examples and draw conclusions about the influence of this angle.

Figure 9 - Weissinger's LLT model.

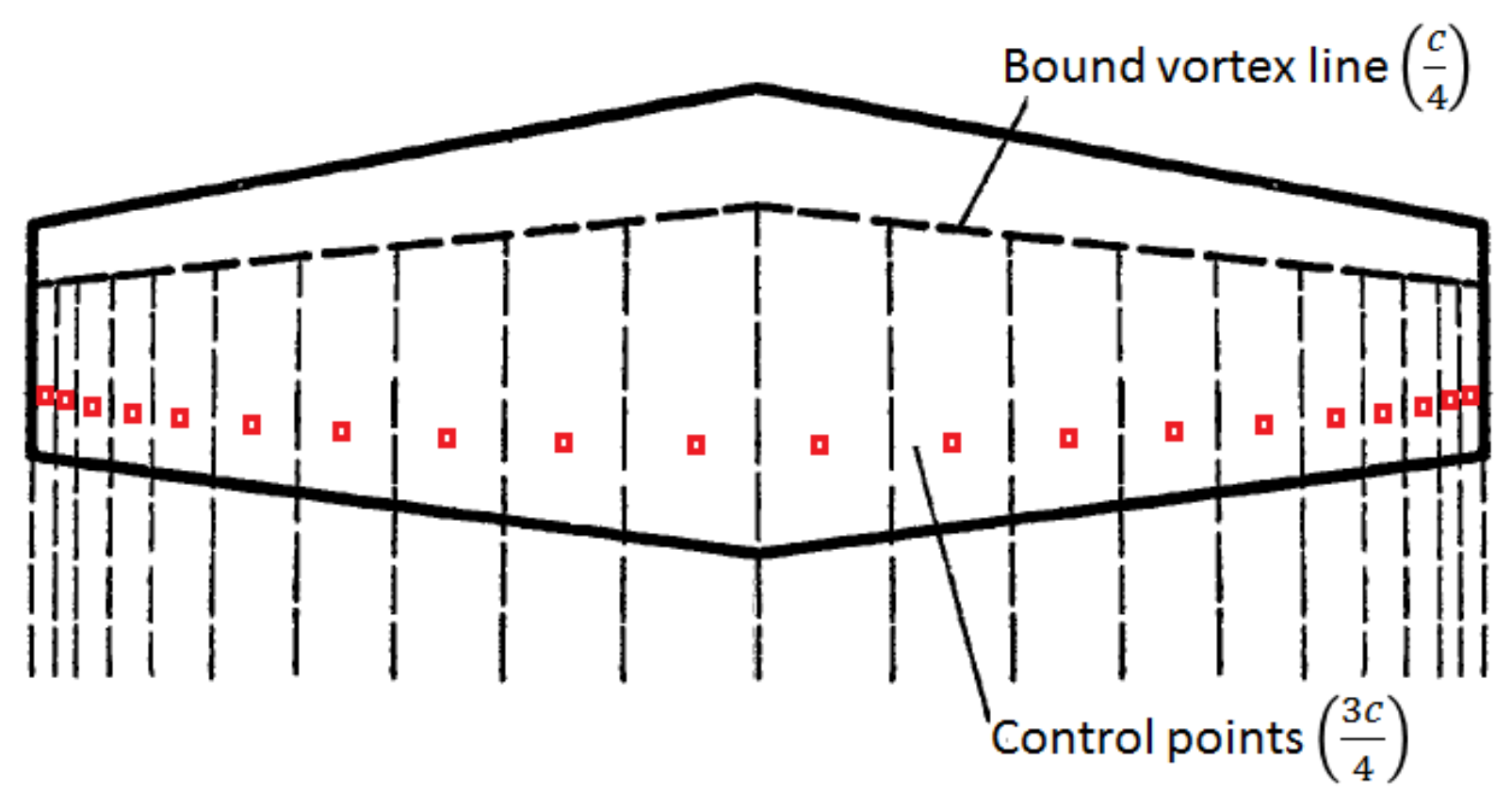

Source: WEISSINGER (1947) (Adapted).

More adaptations to the LLT have been proposed over time, as the one in 1980 proposed by Anderson and Corda, (ANDERSON; CORDA; WIE, 1980) to incorporate viscosity on the overall lift and drag through a nonlinear iterative scheme: data from real airfoils were used to calculate section forces, which were later compared to their potential counterparts from the KJT, and if the two estimates were different, the circulation distribution and the effective angle of attack $\alpha_{e f f}$, were updated based on this difference. 
The authors reported agreement between numerical and experimental data to a maximum relative difference of $20 \%$, and this percentage reduced with the reduction of the incidence AoA; at the stall, however, they found nonunique solutions.

Like Anderson and Corda, in 1996 Pepper and van Dam sought improvements on the WLL, but through the incorporation of section data in Weissinger's formulation (PEPPER; DAM, 1996): they compared the slope of both potential and viscous lift curves at each wing section and suggested that more accurate results could be obtained by making them close to each other through one of the two approaches: by (i) changing the local inflow angle of attack or (ii) the Control Point (CP) chordwise locations. Following these authors' idea, in 1998 Owens incorporated section data in a variant of Weissinger's formulation (OWENS, 1998) - in which the HSVs were placed in vortex-step fashion; coined as Weissinger's nonlinear lifting-line model, the nonlinear iterative scheme relaxed the PBC while did the same force comparison proposed by Anderson and Corda. Owens accurately predicted the aerodynamic forces of tapered and swept wings, finding differences between numerical and experimental data not higher than $2 \%$ for $C_{L}$ and 1 deg for the stall angles. Nonetheless, no unique solution was found for post-stall regimes as well.

Phillips and Snyder took advantage of the vortex-lattice model to represent the circulation distribution by discrete values instead of casting it into a Fourier series (PHILLIPS; SNYDER, 2000); however, they placed the CP along the quarter-chord line, which on one hand dismisses the need of the $\mathrm{PBC}$, but on the other compromises the ability to simulate wings with several geometric characteristics. Viscous effects were incorporated in a fashion similar to Anderson's, but the circulation distribution was updated through Newton's method, allegedly making the numerical method faster and more robust. As expected, and contrary to the authors' proclaim, the chosen CP location makes the method inapt for wings with nonstraight BV lines due to the dramatic increase of the induced velocities with grid refinement (HUNSAKER, 2011; CHREIM et al., 2017).

While both approaches of Pepper and van Dam should in practice produce the same outcome, the second seems more adequate, since inconsistent conclusions have been drawn about the first one (ORTEGA; GIRARDI; KOMATSU, 2004; GALLAY; GHASEMI; LAURENDEAU, 2014). In his MSc thesis, Souza implemented the iterative Pepper and van Dam's change in the CP location in a WLL adapted for flapped wings. The formulation also accounted for an adjustable wake shedding angle, which made the simulation of low aspect ratio wings possible $(A R \leq 4)$. The results presented appeared to be satisfactory, but the same question about grid independence for Phillips and Snyder could be raised, as the method also becomes unstable (but for different reasons) as the grid is refined.

The LLT is an elegant theory that has many applications other than wings, trend that justifies the unceasingly research on the theme: literature has been shown its application for kites, hydrofoils, rotating wings, wind and tidal turbines, aeronautical and 
marine propellers, and even as part of more sophisticated numerical tools; along time, the formulations have been either gradually improved or adapted to attend desired purposes mainly because the simulation domain is relatively simple and the results are reasonably accurate for the low computational time required. In the propeller case, this is not different, provided the appropriate assumptions are made.

\subsubsection{Propeller Lifting-Line Theory}

Both PLL and WLL have been developed concomitantly, meaning they could benefit from mutual development such as Prandtl's hypothesis and the use of sectional data - which makes a vast resource of aeronautical data available for propellers (CARLTON, 2019). However, contrary to the original conception of the WLL, the PLL was initially thought for propeller design, i.e., for a given advance coefficient $J$ and thrust, blade characteristics such as (normalized distributions of) pitch $\left(\frac{P}{D}\right)$, maximum thickness $\left(\frac{t_{0}}{c}\right)$, maximum curvature $\left(\frac{f_{0}}{c}\right)$, and chord $\left(\frac{c}{D}\right)$ were calculated in an attempt to optimize the geometry for minimum torque.

The application of Prandtl's method to propellers led to the important conclusion that the induced section drag is a function of the design conditions, which led Betz to establish a basic minimum energy loss condition by analyzing the vortices in the slipstream of a lightly-loaded propeller with an infinite number of blades in a uniform flow; he found that this condition happened when the far wake had a constant radial pitch and that the induced velocities along the blades were normal to the inflow velocity (Betz's condition of normality - figure 10) (CARLTON, 2019). Additionally, as this model assumed an infinite number of blades to circumvent the complex wake geometry, Prandtl helped Betz establish a correction of the results to propellers with few blades.

Figure 10 - Betz's Condition of Normality: $\vec{V}_{I n d}$ is perpendicular to $\vec{V}_{R}$

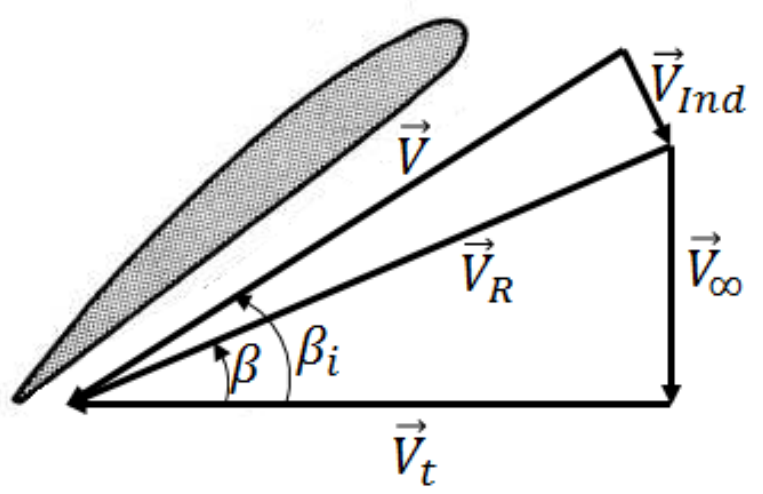

Source: Author

In 1936, Kawada derived analytic expressions for the velocity induced by infinitelength helical vortices (KAWADA, 1936), which was an advance to the PLL, as during 
lightly and moderately-loaded conditions the assumption of constant radial pitch is held; these expressions made possible the application of the helical vortices to model propellers with finite number of blades, as Kawada did by representing each blade as a line of constant vorticity extending from root to tip and TVs shed at these locations, so the hub influence is the sum of all root TVs.

What has become regarded the classic representation of the lifting-line for propellers was developed in 1952 by Lerbs and followed the work of Kawada (CARLTON, 2019): Lerbs proposed a more representative formulation in which each blade was modeled by the superposition of Helical Horseshoe vortex (HHSV) - in a similar fashion to Prandtl - and the hub, modeled as an infinitely long cylinder, considered to have zero circulation along its surface (LERBS, 1952). The author derived expressions for the induced velocities in terms of potential functions and observed that they were affected by the existence of singularities, so he introduced induction factors that circumvented them. Lerbs applied his method to three different problems: (i) free-running optimum propellers with and without the presence of hub, assessing the influence of this geometry on the circulation distribution and the induced velocities; (ii) non-optimum propellers, determining the induced velocities as function of the thrust loading coefficient, circulation distribution, and advance coefficient (and also the inverse problem, i.e., determining the circulation distribution and the induced velocities as function of the propeller geometry and the advance coefficient); and (iii) propellers operating in radially varying wakes.

The LLT of Lerbs has been used ever since without many changes, and the few important ones were oriented towards the design of propellers; in 1957, for instance, Wrench derived expressions that improved the estimates of Lerb's Induction Factors and thus obtained expressions for the velocity induced by $Z$ helical vortices as function of a hydrodynamic pitch angle (JR, 1957). The author concluded that Lerbs's numerical values for the induction factors were generally correct to within two units in the third decimal place. In 1968, Morgan proposed LST corrections factors for camber and ideal angle due to loading and due to thickness that improved the LLT (MORGAN; SILOVIC; DENNY, 1968); he showed that the camber and ideal angles were generally greater in 3-D case than in 2-D to produce the same lift coefficient and that the correction factors in general increased with increasing Expanded Area Ration (EAR), while those for camber and ideal angle due to loading decreased with increasing number of blades. He also found that thickness, in general, induced positive (and increasing with the number of blades) angles that required corrections to the blade pitch, and finally, that skew induced inflow angles that needed positive pitch changes toward the blade root and negative ones toward the blade tip.

To improve Lerbs' hub model, Kerwin proposed a model based on image vortices (KERWIN; LEOPOLD, 1964) that allegedly works remarkably well (KERWIN; HADLER, 
2010); although no simple image exists for helical vortices, infinite straight vortices have images whose location inside an infinite cylinder can be analytically obtained so the combined normal velocity over the cylinder surface is zero - Figure11. Assuming the same location for starting the helical image vortices, the hub is modeled model by their superposition. Kerwin observed that the circulation at the hub had a finite value with near-zero derivative in the radial direction and that the agreement with more complex models from potential flows was almost exact. The finite circulation at the hub region created a concentrated vortex that must be shed downstream, following Helmholtz's and Kelvin's theorems and that physically happens (Figure 12). This effect reduces the local pressure, which is responsible for a pressure drag that affects the net propeller thrust, and whose value was estimated by Wang in 1985 (WANG, 1985).

Figure 11 - Infinite straight vortex and its image inside the cylindrical hub.

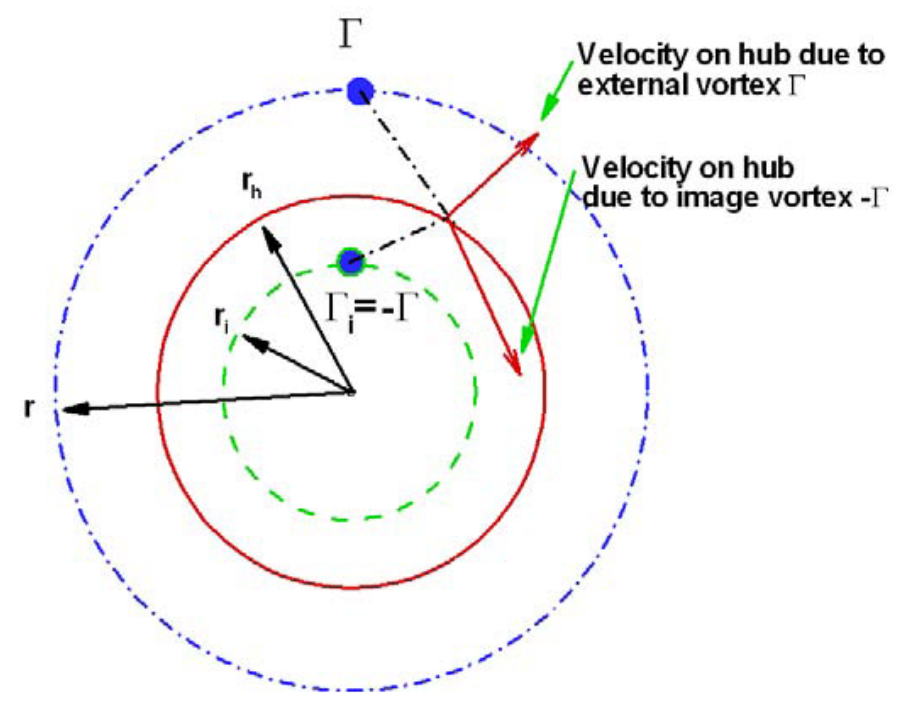

Source: KERWIN; HADLER, 2010 (Adapted).

At the Massachusetts Institute of Technology, researchers continued to work on the LLT of Kerwin either through adaptations or applications for specific purposes: in 2007, Chung revised the hydrodynamic propeller design problem and extended it to perform more sophisticated design applications such as ducted and contra-rotating propellers (CHUNG, 2007); in 2008, Stubblefield used the formulation to model axisymmetric-ducted propellers with no gap between the geometries: he implemented and analyzed two subroutines for determining the optimum circulation distribution and latter validated the best one (STUBBLEFIELD, 2008), along with implementing the optimization routine; in 2009, Flood extended the method for propeller analysis thus allowing the use of the formulation to off-design conditions, which he showed to have good agreement with experimental data (FLOOD, 2009); finally, in 2010, Epps used the propeller tool to analyze propeller 
Figure 12 - Propeller evidencing hub and tip vortices.

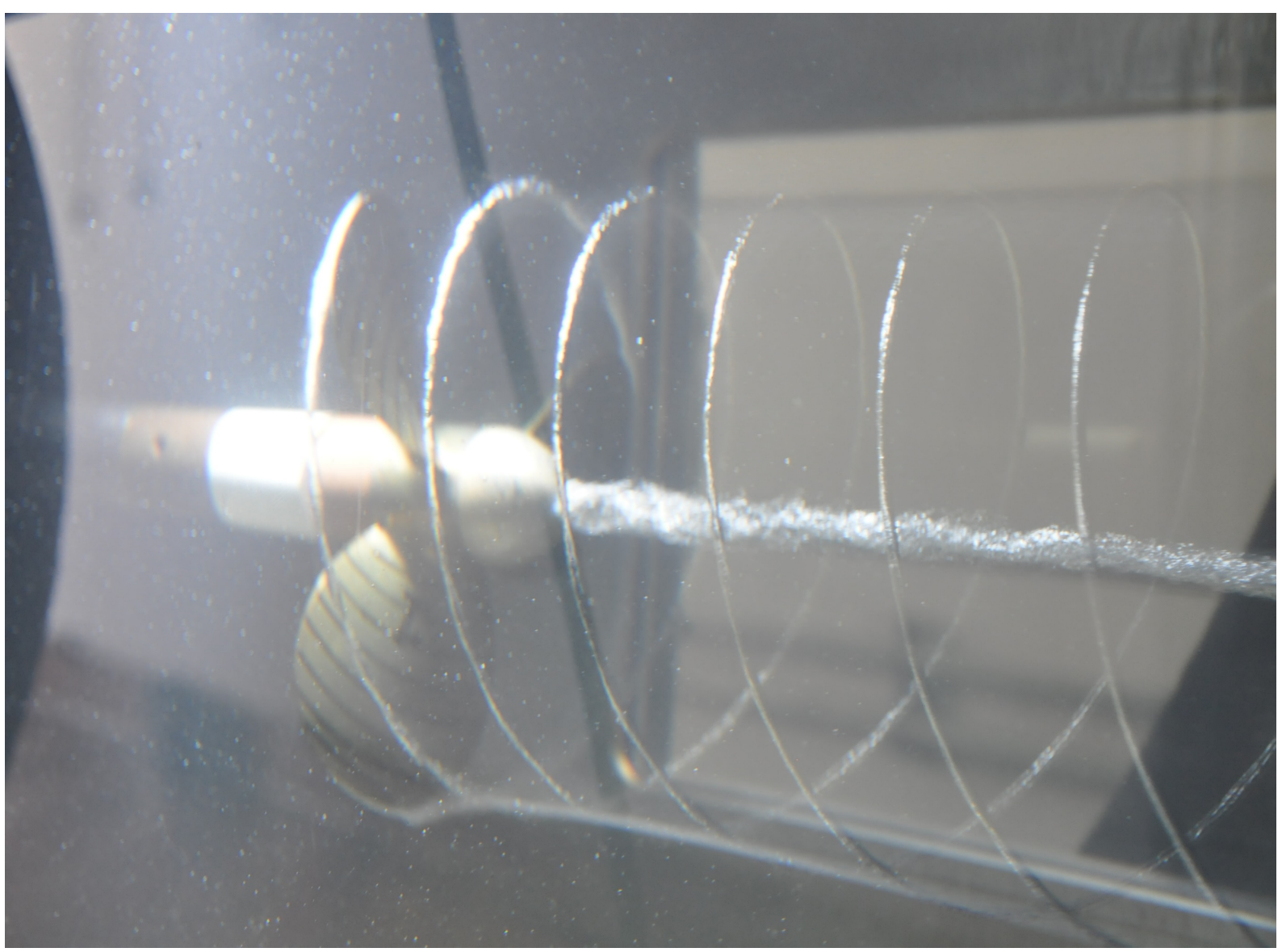

Source: Author

loads during steady-state operation: he used the LLT formulation, a special case of the general impulse framework of his thesis, to design, build and test a propeller over a range of off-design operating conditions. He found that the experimental results matched the predicted performance curve, thus providing important validation data for his work (EPPS, 2010). This variety of works coalesced into an open-source LLT-LST hybrid model for design and analysis of marine propellers and horizontal-axis turbines named OpenProp (EPPS; KIMBALL, 2013) with capabilities such as design optimization, performance analysis, and CAD geometry generation.

Openprop is constantly under improvements, and in 2017 Epps reconsidered the classic wake model due to its theoretical inconsistency in the induced velocities when the PLL is approximated to an actuator disc (EPPS, 2017). His proposed model, in addition to being consistent, was shown to be computationally more stable at bollard pull conditions. Also, while both models presented almost identical results in uniform flow with approximately constant hydrodynamic pitch angles, they produced different outcomes for nonuniform flows.

IndFact is another LLT-LST formulation for propeller design that is based on Lerbs' 
Figure 13 - Lerb's and Epps' wake models. Note the similarity with the superimposed and vortex-step formulations, respectively.
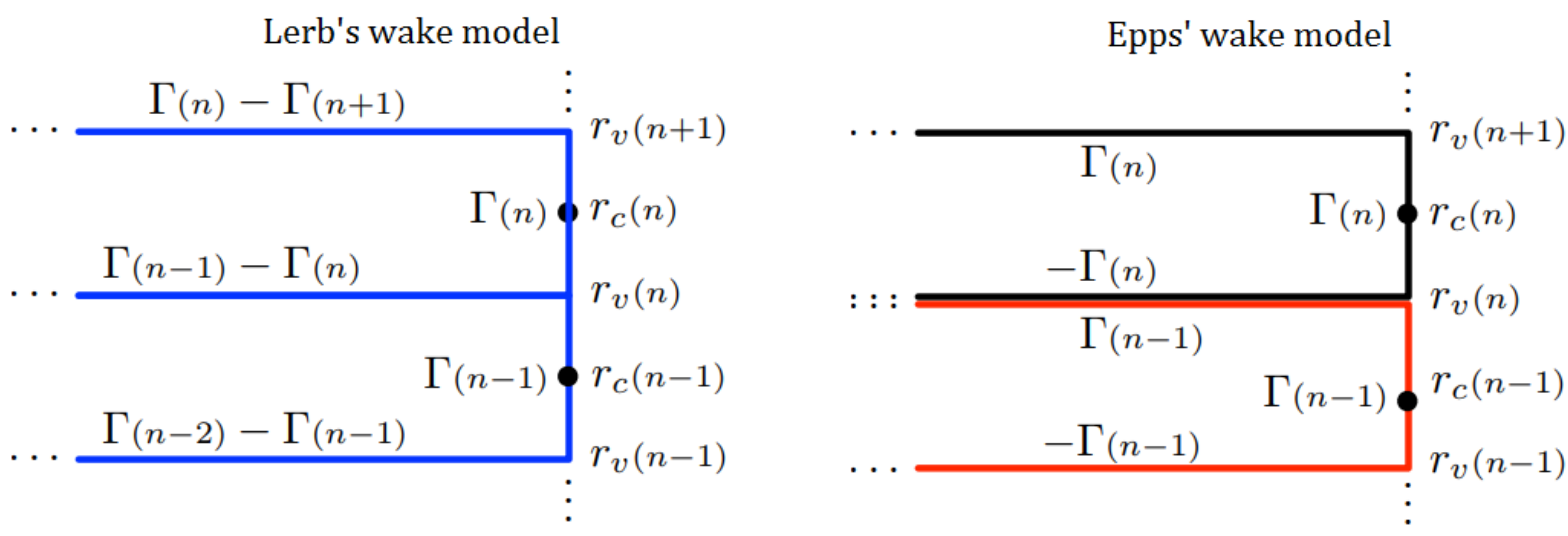

Source: EPPS, 2017 (Adapted).

induction factors and the correction polynomials of Morgan; the method, developed by the Maritime Research Institute Netherlands, initially uses the LLT to obtain a propeller geometry through iteratively converging the hydrodynamic forces and the blade cavitating area, and subsequently uses the LST factors to correct it. (MARIN, 2010). While only a little information is given about the method capabilities - such as simulating raked and skewed propellers -, the authors state that IndFact accuracy is of a few percents.

Most of what has been documented about PLL methods are based on Lerbs' work, and without the LST coupling, the results obtained are in general not satisfactory, in addition to the limitations of simulating propellers without rake or skew under potential flow regimes - even though viscosity has somewhat simplistically been included (KERWIN; HADLER, 2010). The introduction superficially presented a tendency on the use of this low-cost method for a variety of applications, and also explored some of the extensions that happened along with the history of the WLL; likewise, such extensions can be applied to a novel PLL formulation if it is developed correspondingly. This novel formulation and its expected advantages will be presented in the next chapters. 


\section{Mathematical and Physical Models}

Wing and propeller lifting-lines share the same foundations as both geometries are represented by HSVs and must, either directly or not, satisfy both force equivalence and the PBC. The differences lie on the particularities of the geometries: first, while for wings a wall or symmetry condition usually suffices to account for the presence of the fuselage on the proper estimation of Lift and Drag, for propellers the hub must be more carefully modeled given its size and orientation relative to the blades; second, while the far wake of wings is usually parallel to the freestream direction - thus modeled by a series of Straight Horseshoe Vortex (SHSV) -, the propeller counterpart, under certain conditions, follows an almost helical path of constant hydrodynamic pitch angle $\beta_{N}$ - thus modeled by HHSV -; finally, while the wing sections stay on a cartesian plane, blade sections lie over cylindrical surfaces - Figure 14.

Figure 14 - Wing and blade sections representations.
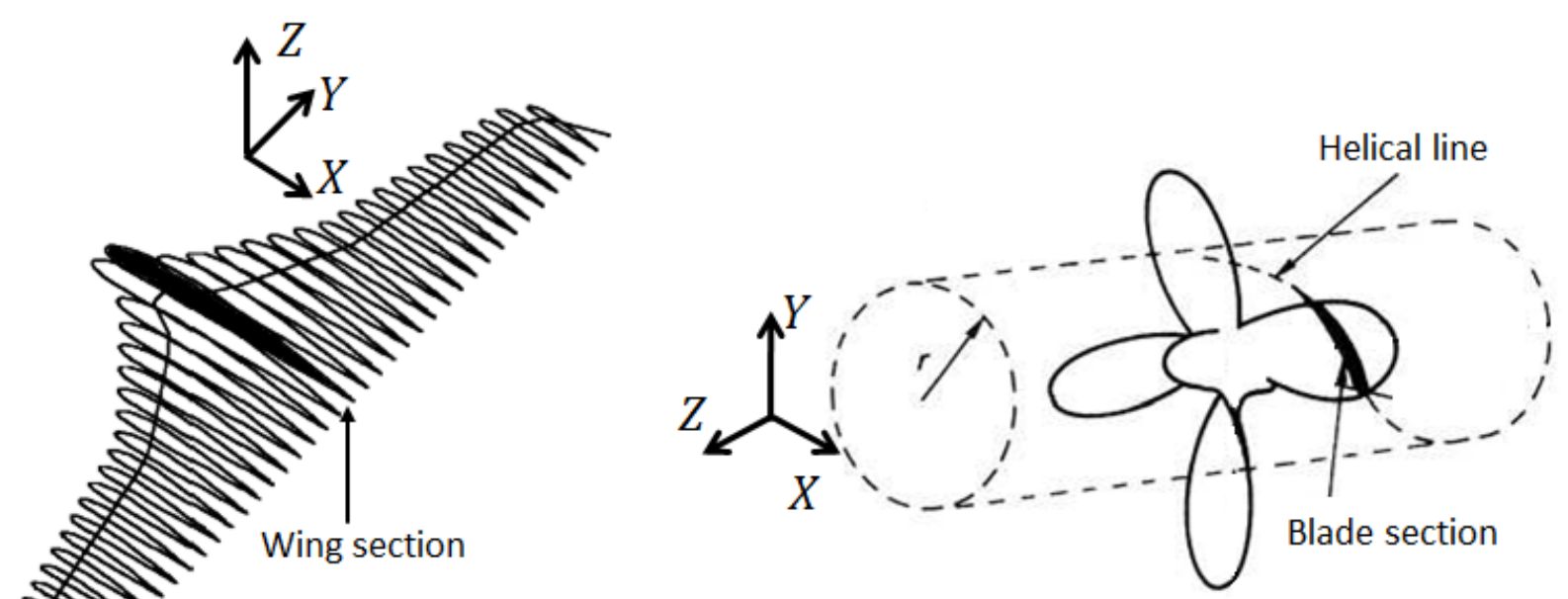

Source: Author (Adapted from WICKENHEISER; GARCIA, 2007 and and CARLTON, 2019) .

Since the flow over a wing is simpler, the WLL formulation will be presented first; after being extensively discussed, it will be adapted (due to their geometric differences) to the propeller case. As expected, both formulations are based on a nonlinear scheme that departs from a linear solution and, iteratively, incorporates the influence of viscosity on the aerodynamic/hydrodynamic forces and moments. 


\subsection{Wing Lifting-Line}

The following lifting-line formulation is mainly based on the work of Katz (KATZ; PLOTKIN, 2001), for the general wing modeling and linear scheme, and on the works of Pepper and van Dam (PEPPER; DAM, 1996) and de Souza (SOUZA, 2005) for the nonlinear, iterative scheme. The wingspan is modeled by a series of $N$ SHSV, whose geometry choice is addressed in the appendix, placed alongside in a Vortex-Step fashion (Figure 15).

Figure 15 - Several views of the WLL discretization. Left - upper, Top - back, Down side, Right - 3-D.
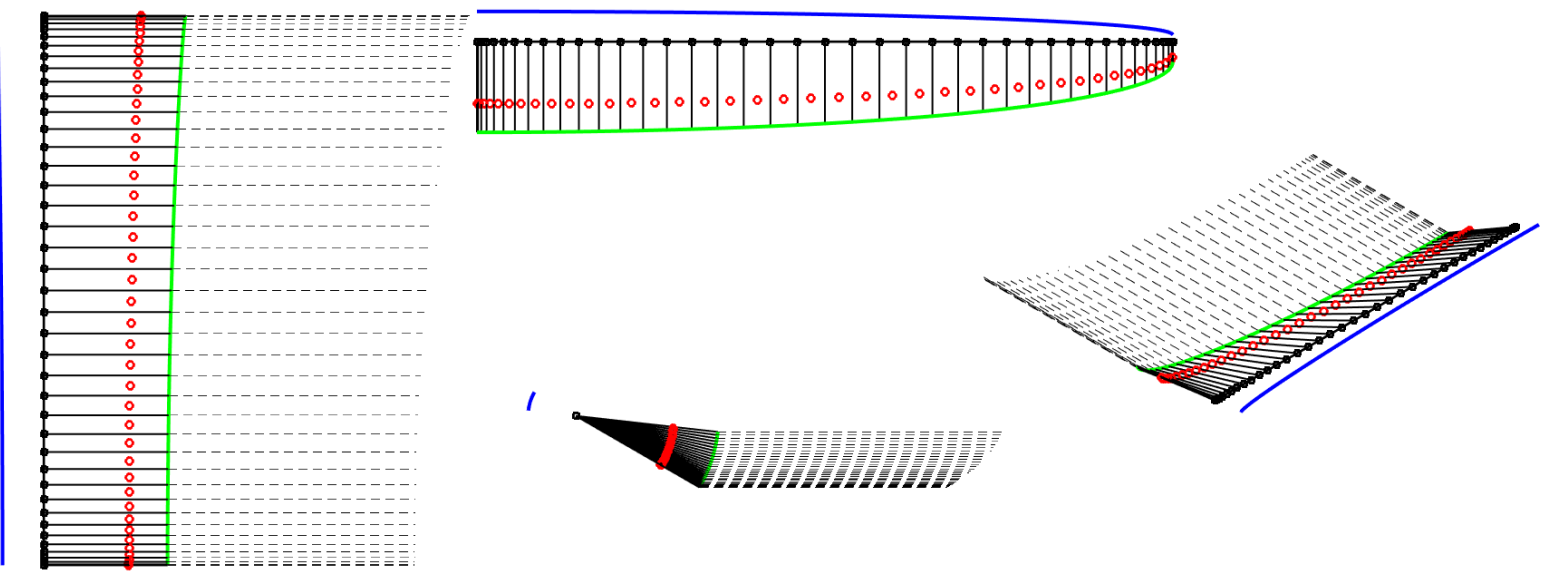

Source: Author

Figure 16 presents an isolated horseshoe vortex. The velocity $\vec{V}_{V S_{i j, k_{j} k_{j}+1}}$ induced by one of its segments, with circulation $\Gamma_{j}$, at a point $i$ in space is (PHILLIPS; SNYDER, $2000)$ given by equation (2.1), in accordance to the parameters defined in figure 17 :

$$
\vec{V}_{V S, i j, k_{j} k_{j}+1}=\frac{\Gamma_{j}}{4 \pi} \frac{\left(r_{i j, k_{j}}+r_{i j, k_{j}+1}\right)\left(\vec{r}_{i j, k_{j}} \times \vec{r}_{i j, k_{j}+1}\right)}{r_{i j, k_{j}} r_{i j, k_{j}+1}\left(r_{i j, k_{j}} r_{i j, k_{j}+1}+\vec{r}_{i j, k_{j}} \cdot \vec{r}_{i j, k_{j}+1}\right)}
$$

Thus, the overall induced velocity of the $\mathrm{j}^{\text {th }}$ horseshoe vortex over $i$ is the superposition of the five segments, as it is assumed that the starting vortex is sufficiently far from this point.

$$
\vec{V}_{S H S V, i j}=\sum_{k_{j}=1}^{5} \vec{V}_{V S, i j, k_{j} k_{j}+1}=\frac{\Gamma_{j}}{4 \pi} \vec{v}_{W, i j}
$$

in which:

$$
\vec{v}_{W, i j}=\sum_{k_{j}=1}^{5} \frac{\left(r_{i j, k_{j}}+r_{i j, k_{j}+1}\right)\left(\vec{r}_{i j, k_{j}} \times \vec{r}_{i j, k_{j}+1}\right)}{r_{i j, k_{j}} r_{i j, k_{j}+1}\left(r_{i j, k_{j}} r_{i j, k_{j}+1}+\vec{r}_{i j, k_{j}} \cdot \vec{r}_{i j, k_{j}+1}\right)}
$$


Figure 16 - Horseshoe model used in the wing formulation of the lifting-line problem.

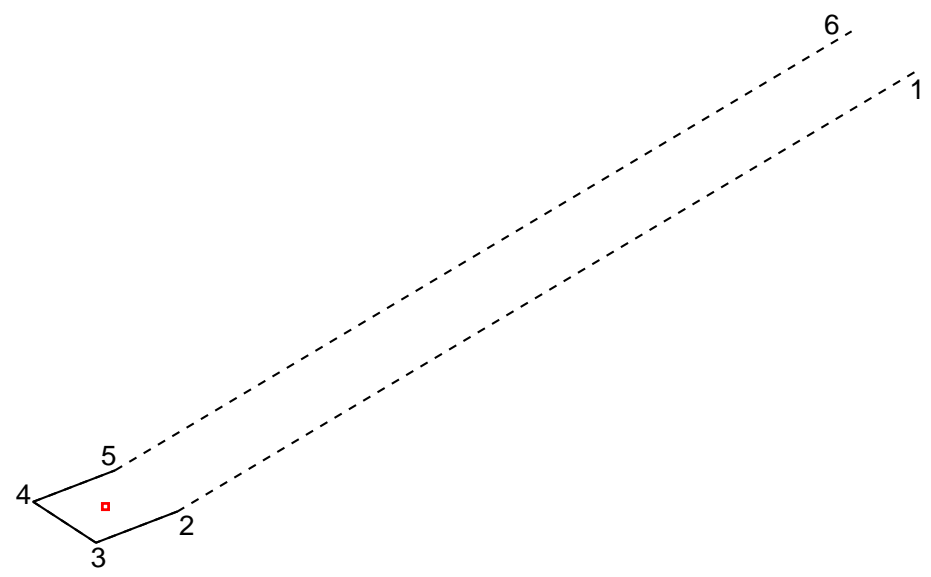

Source: Author.

Figure 17 - Straight vortex segment with circulation strength $\Gamma_{j}$

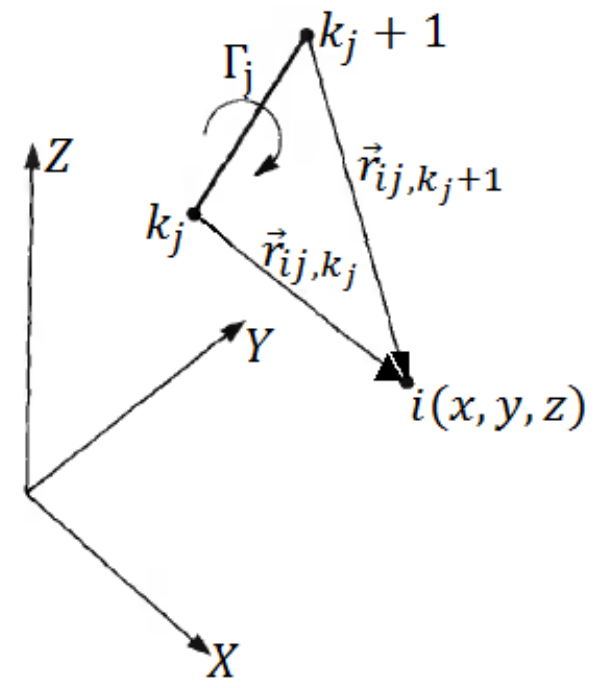

Source: KATZ; PLOTKIN, 2001 (Adapted).

The total velocity at $i$ is the superposition of the $N$ SHSV contributions and the freestream velocity $\vec{V}_{\infty}$ :

$$
\vec{V}_{W, i}=\vec{V}_{\infty}+\sum_{j=1}^{N} \vec{V}_{S H S V, i j}
$$

The normal-to-the-wing component of $\vec{V}_{W, i}$ is zero as a consequence of the PBC: 


$$
\begin{gathered}
\hat{u}_{n, i} \cdot \vec{V}_{W, i}=0 \rightarrow \\
\hat{u}_{n, i} \cdot \vec{V}_{\infty}+\sum_{j=1}^{N} \hat{u}_{n, i} \cdot \vec{V}_{S H S V, i j}=0 \rightarrow \\
\sum_{j=1}^{N} \hat{u}_{n, i} \cdot \vec{V}_{S H S V, i j}=-\hat{u}_{n, i} \cdot \vec{V}_{\infty}
\end{gathered}
$$

with $\hat{u}_{n, i}$ as the $\mathrm{i}^{\text {th }}$ normal unit vector. With the aid of equation (2.2), equation (2.1) can be more conveniently written:

$$
\begin{gathered}
\sum_{j=1}^{N} m_{i j} \Gamma_{j}=-4 \pi \times W_{\infty, i} \\
m_{i j}=\hat{u}_{n, i} \cdot \vec{v}_{W, i j} \\
W_{\infty, i}=\hat{u}_{n, i} \cdot \vec{V}_{\infty}
\end{gathered}
$$

Equation (2.6) is imposed for the $N$ corresponding control points, which results in a system of $N \times N$ equations that can be written in a matrix form:

$$
\mathbb{M} \Gamma=-\mathbf{W}_{\infty}
$$

\subsubsection{Linear scheme}

The $m_{i j}$ are the elements of $\mathbb{M}$, and the circulation distribution $\boldsymbol{\Gamma}$ is obtained from the solution of (2.7). With this solution, the aerodynamic potential forces are calculated with the aid of a three-dimensional version of the KJT (PHILLIPS; SNYDER, 2000), under the assumption of Prandtl's hypothesis, and considering only the influence of the trailing vortices (KATZ; PLOTKIN, 2001).

$$
\begin{gathered}
\delta \vec{F}_{W, i}=\rho \vec{V}_{V T W, i} \times\left(\Gamma_{i} \delta \vec{l}_{i}\right) \rightarrow \\
\delta F_{W, i}=\rho \Gamma_{i} V_{V T W, i} \delta l_{i} \sin \psi_{i} \\
\vec{V}_{T V W, i}=\vec{V}_{\infty}+\sum_{j=1}^{N}\left(\vec{V}_{W H S_{i j}}-\vec{V}_{V S_{i j, 34}}\right)
\end{gathered}
$$

$\delta \vec{l}_{i}$ is the $\mathrm{i}^{\text {th }}$ bound vortex vector length, and $\psi_{i}$ is the angle between this vector and $\vec{V}_{V T W, i}$. The forces are them normalized to obtain the corresponding section aerodynamic coefficients; from the standard definition of the aerodynamic normal section force coefficient $C_{n}$, a potential equivalent of this quantity $\left(C_{n W P o t, i}\right)$ is obtained: 


$$
\begin{array}{r}
\frac{\delta F}{\delta l} \equiv \frac{1}{2} \rho V_{W}^{2} c C_{n} \\
C_{n W P o t, i}=\frac{\delta F_{W, i}}{\frac{1}{2} \rho V_{W, i}^{2} c_{i} \delta l_{i}}
\end{array}
$$

\subsubsection{Nonlinear scheme}

The present nonlinear scheme is necessary to incorporate viscosity on lift and drag by introducing real foil data rather than using potential 2-D flow results; the PBC was derived under the assumption of the TFT, but if a foil is chosen such that it has a different lift curve slope $\frac{\partial C_{n}}{\partial \alpha}$, then the scheme adjusts the CPs location at which this BC is satisfied. For the $\mathrm{i}^{\text {th }} \mathrm{CP}$ chordwise location, having chord length $c_{i}$ :

$$
x_{c p, i}=\left[\frac{1}{2} \frac{\frac{\partial C_{n, i}}{\partial \alpha_{i}}}{2 \pi}+\frac{1}{4}\right] c_{i}
$$

while the spanwise $y_{c p_{i}}$ and normal $z_{c p_{i}}$ coordinates are adjusted accordingly so the control point remains over the planform. In practice, a change in the CP location alters the slope of the $C_{n} \times \alpha$ curve (Figure 18); thus, after $C_{n W P o t, i}$ is obtained from the linear scheme as an initial solution guess, $\frac{\partial C_{n, i}}{\partial \alpha_{i}}$ is assumed to be $2 \pi$. Next, real foil data (numerical or experimental) are used to estimate $C_{n W V i s, i}$ and $\frac{\partial C_{n, i}}{\partial \alpha_{i}}$ Vis as functions of the effective angle of attack $\alpha_{e f f, i}$ (from the knowledge of $\frac{\partial C_{n, i}}{\partial \alpha_{i}}$ ) and local Reynolds $R e_{i}$. In practice, this has the same effect as including viscosity in the vortices strengths (or, equivalently, in the induced velocities).

$$
\begin{gathered}
\alpha_{e f f, i}=\frac{C_{n i_{W P o t}}}{\frac{\partial C_{n_{i}}}{\partial \alpha_{i}}}-\alpha_{L 0_{i}} \\
C_{n W V i s, i}=C_{n W}\left(\alpha_{e f f, i}, R e_{i}\right) \\
\frac{\partial C_{n, i}}{\partial \alpha_{i} V i s}=\frac{C_{n W V i s, i}}{\alpha_{e f f, i}-\alpha_{L 0, i}}
\end{gathered}
$$

$C_{n W V i s, i}$ and $C_{n W P o t, i}$ are compared to each other and if their difference, either absolute or relative, is not within a desirable tolerance, $\frac{\partial C_{n_{i}}}{\partial \alpha_{i}}$ is updated according to equation (2.14):

$$
\frac{\partial C_{n, i}}{\partial \alpha_{i}}=(1-\Omega) \frac{\partial C_{n, i}}{\partial \alpha_{i}}+\Omega \frac{\partial C_{n, i}}{\partial \alpha_{i} \text { Vis }}
$$

in which $\Omega$ is an under relaxation factor, typically assumed to be 0.8 for most of the cases (although for harder-to-converge cases, this value could be as low as 0.2 ). The linear 
Figure 18 - Scheme for the nonlinear formulation. The control points locations change the slope $\frac{\partial C_{n}}{\partial \alpha}$ of the curves accordingly.

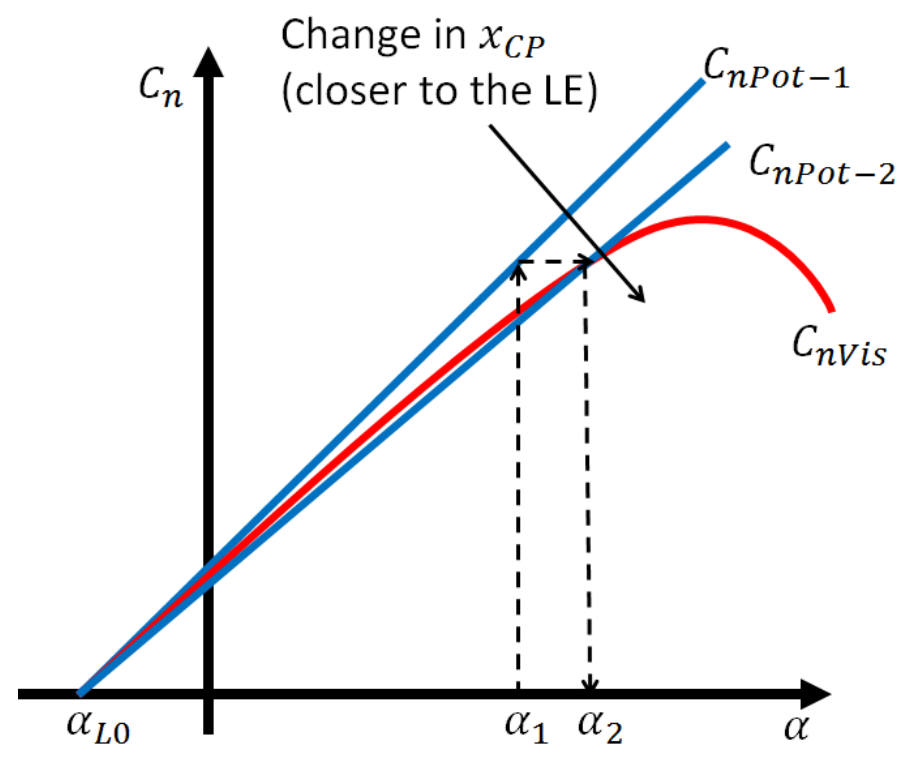

Source: Author

scheme is repeated to ensure that PBC is satisfied for the new locations, thus obtaining new values for $\Gamma_{i}$ and $C_{n P o t}, i$; the iterative process repeats until convergence, which usually takes about 10 to 20 iterations (and for harder-to-converge cases, such number can go up to 100$)$.

The proposed nonlinear formulation is restricted to the near-to-linear regions of the $C_{n} \times \alpha$ curves, as from equation 2.13, the slope is assumed constant. Additionally, after convergence, the sectional viscous axial force coefficient $C_{c, i}$ is retrieved from the same database, as it is only necessary for post-processing calculations.

\subsubsection{Post-processing and flowchart}

After numerical convergence, several variables of interest can be calculated, such as the final circulation distribution spanwise, the distribution coefficients of lift and drag $\left(C_{l}\right.$ and $\left.C_{d}\right)$ and the overall wing lift and drag coefficients $\left(C_{L}\right.$ and $\left.C_{D}\right)$. Particularly, the last two variables are obtained from $C_{n}$ and $C_{c}$ distributions: for each location, the forces must be transformed from the foil reference frame to the wing counterpart, which is done by an $\alpha_{r o t, i}=\alpha+\theta_{i}-\alpha_{e f f, i}$ rotation about the span axis, with $\theta$ the geometric twist and $\alpha$ the freestream AoA. 


$$
\begin{aligned}
C_{L} & =\frac{1}{V_{\infty}^{2} S} \sum_{i=1}^{N} V_{W, i}^{2} \delta A_{i}\left[C_{n, i} \cos \left(\alpha_{r o t, i}\right)-C_{c, i} \sin \left(\alpha_{r o t, i}\right)\right] \\
C_{D} & =\frac{1}{V_{\infty}^{2} S} \sum_{i=1}^{N} V_{W, i}^{2} \delta A_{i}\left[C_{n, i} \sin \left(\alpha_{r o t, i}\right)+C_{c, i} \cos \left(\alpha_{r o t, i}\right)\right]
\end{aligned}
$$

$S$ is the wing planform area and for inviscid cases, $C_{c}=0$, i.e., only the induced drag $C_{D i}$ is calculated. The presence of section viscous drag increases the overall drag while it decreases the lift; thus, its inclusion should increase the accuracy of the hydrodynamic forces and moments, if the flow regime is adequate.

The following flowchart illustrates the WLL pipeline:

Figure 19 - Flowchart of the WLL formulation

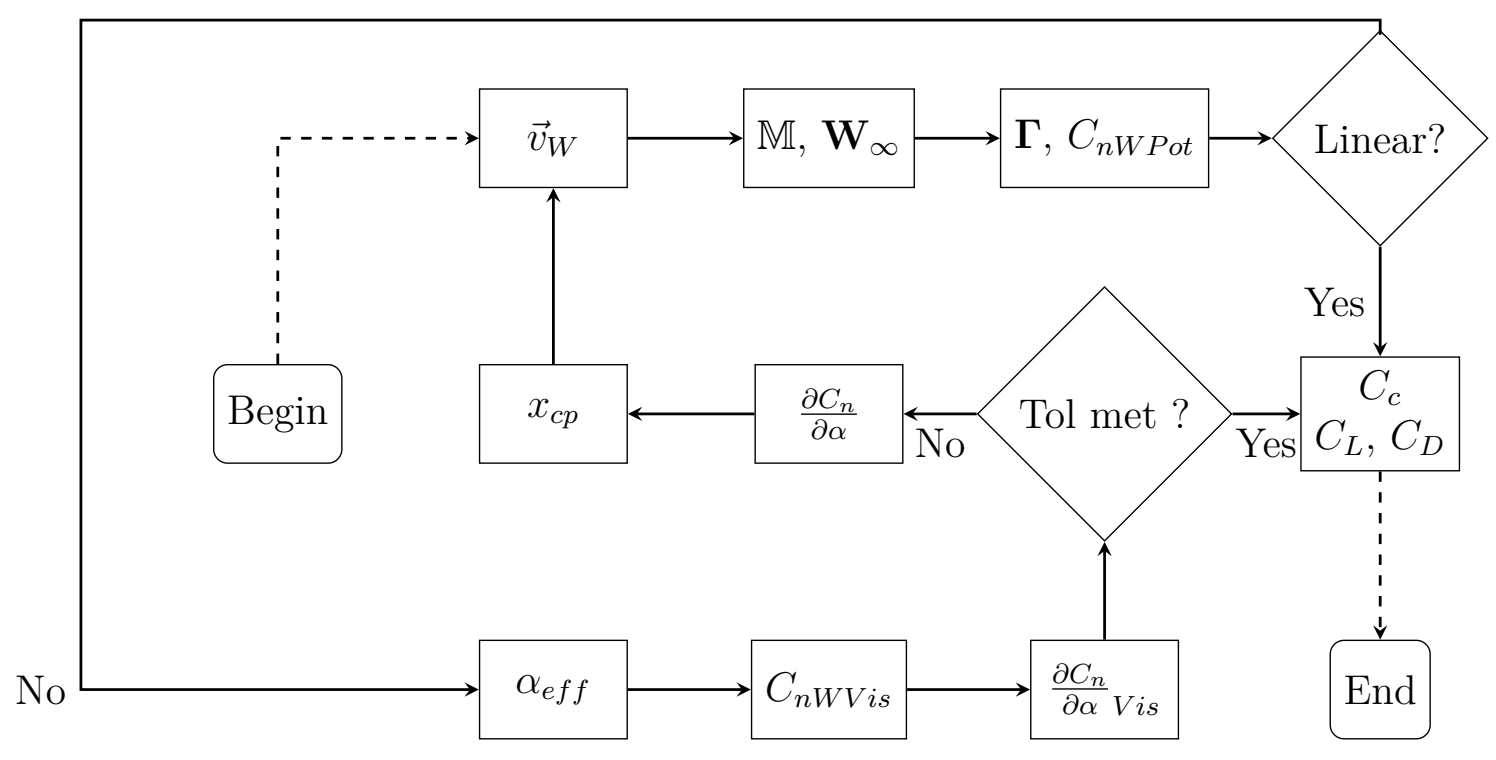

Source: Author

\subsection{Propeller Lifting-Line}

The particulars about operation and geometry differentiate wings from propellers in terms of modeling; while a wing can be understood as a fixed blade that travels parallel to the freestream direction (generating an almost perpendicular aerodynamic force with respect to its direction of motion), the propeller can be understood as a system of several blades that rotate about the freestream direction (generating a hydrodynamic force whose main component is parallel to its direction of motion); thus, instead of a straight plane, the propeller blades convey the fluid through paths dependent on the advance and rotation velocities that, under lightly and moderately loading conditions (which can be 
simplistically categorized in terms of ranges for $C_{T}-0.25$ for lightly, up to approximately 0.7 for moderately, and higher values for heavily (CHUNG, 2007; DINIZ, 2015), or if the terms of order two and higher of the axial and tangential induced velocities are small compared to the advance and rotational velocities, respectively (FLOOD, 2009), which is assumed to happen when $\frac{v_{t}}{V_{t}}$ and $\frac{v_{a}}{V_{\infty}}$ are up to approximately 0.2 ), can be approximated as helical planes with constant hydrodynamic pitch. Figure 20 depicts a typical discretization.

Figure 20 - PLL discretization.

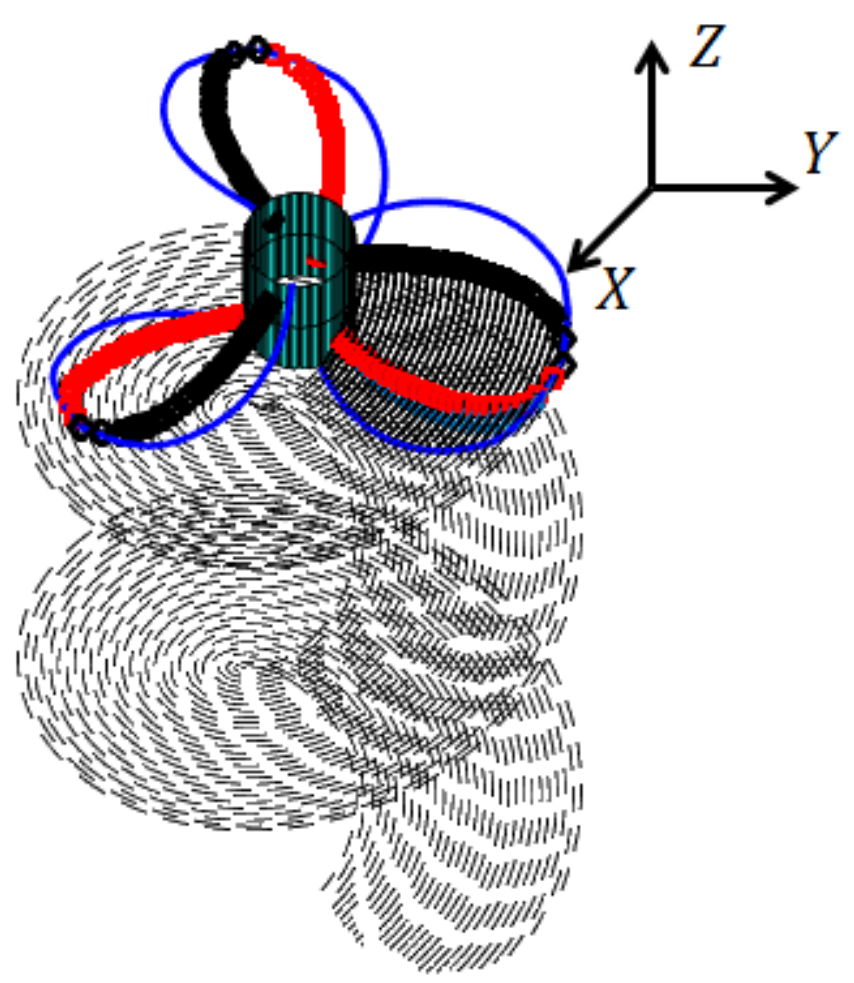

Source: Author

Under such loading conditions, the SHSV is adapted to a close to HHSV - figure 21, with part of the TVs over the blade planform while the other part follows a helical patch with constant pitch axially (but not necessarily radially symmetric, so the formulation can be applied to nonuniform wake conditions). The BV and CP remain at the same $\frac{c}{4}-\frac{3 c}{4}$ initial locations, so the expressions for the induced velocities that are common in the classic formulations (KAWADA, 1936) cannot be used. Alternatively, the HHSV can be approximated a series of straight segments such that, in the limit of an infinite number of them (with infinitesimal-length), this approximation is exact. Assuming a hydrodynamic pitch angle $\beta_{B V}$, the free part of the TV can be written in terms of the bound vortex radius $R_{B V}$, the blade Trailing Edge (TE) coordinates, and a parameter $t>0$ such that: 


$$
\begin{gathered}
x_{T V}=R_{B V} \sin \left[t+\tan ^{-1}\left(\frac{y_{T E}}{x_{T E}}\right)\right] \\
y_{T V}=R_{B V} \sin \left[t+\tan ^{-1}\left(\frac{y_{T E}}{x_{T E}}\right)\right] \\
z_{T V}=t R_{B V} \tan \left(\beta_{B V}\right)+z_{T E}
\end{gathered}
$$

for a Counter Clockwise (CCW) rotation propeller; for a Clockwise (CW) one, the expressions for $x_{T V}$ and $y_{T V}$ interchange. Even though this wake model is computationally more expensive, it allows the use of equation (2.1) for each segment, which dismisses the need for numerical integration and is a precursor of more sophisticated wake models (HUNSAKER, 2011; TIAN; KINNAS, 2012; DINIZ, 2015), making an occasional upgrade easy. On the other hand, the use of more sophisticated ones may not be justified as the current formulation has been developed for up to moderately loading conditions; so, the increase in computations costs may not be accompanied by the respective accuracy gains. The use of free-wake models, however, might improve the results of $K_{T}$ and $K_{Q}$ specially at high loading conditions (DINIZ, 2015).

Figure 21 - Close to HHSV (and its discretization) used in the present PLL, along with the CP. The bold lines are part of the vortex attached to the blade, while the dashed the part shed into the free stream. The red square is a control point.

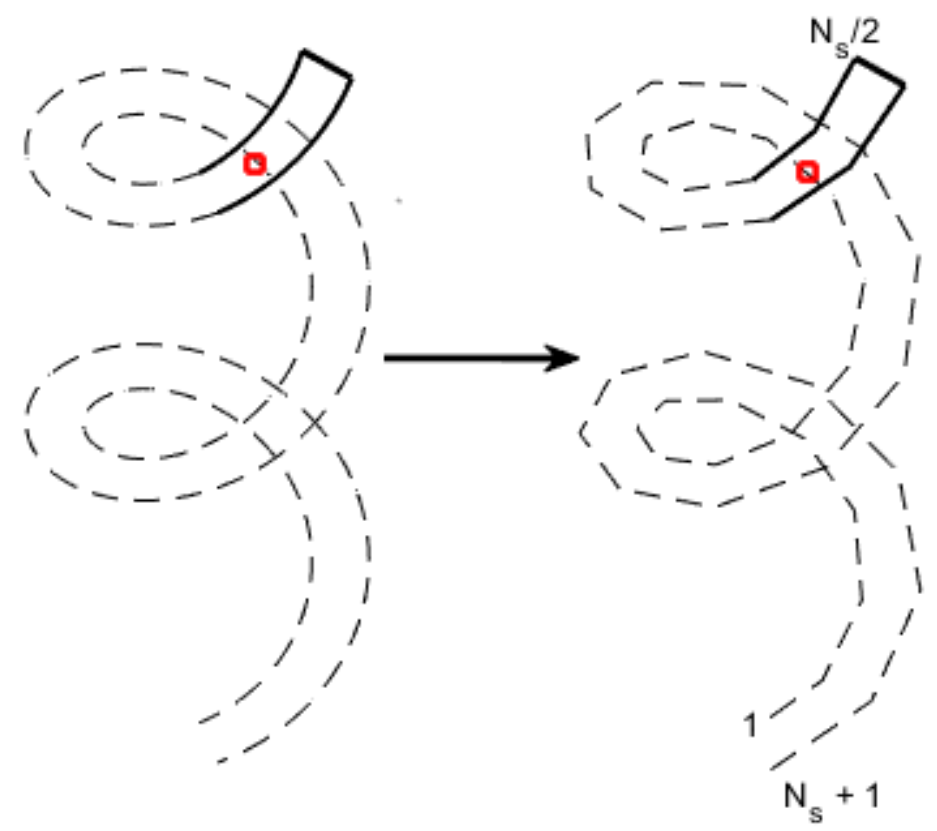

Source: Author

Since the proposed discretization already changes the straight $\frac{c}{2} \mathrm{BV}$ and $\mathrm{CP}$ lines, it itself broadens a restriction of the classic PLL: for propeller design, as the thrust is generated solely by the blades camber distribution as a consequence of the shock-free entrance requirement, the $\frac{c}{2}$ line assumption is adequate since in this case, the centers of 
pressure of the foils move towards $\frac{c}{2}$; in an analysis context, however, the $\frac{c}{4}$ representation is more appropriate because the shock-free entrance is no longer a requirement, so for most of $J$ ranges, the blade sections have nonzero effective angles of attack, making their centers of pressure move towards this location (figure 22) (KATZ; PLOTKIN, 2001).

Figure 22 - Projected area of a blade with no rake or skew. In this case, the directrix (in green) is the chordwise symmetry axis.

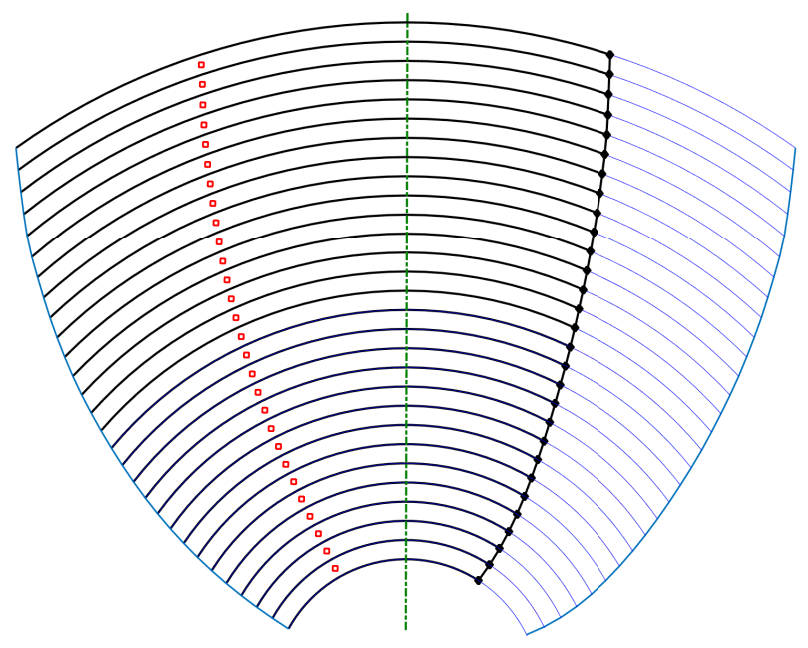

Source: Author

The more fundamental expressions are the same for both WLL and PLL, thus in order to make distinction of the blade influences on one another, which is particularly important for nonuniform wake analysis, upper-indices $b_{i}$ and $b_{j}$ were added to refer to the blades interactions. Thus, given a propeller rotating at an angular rate $\vec{\omega}$, whose $Z$ blades are each modeled by $N$ HHSVs that have, each, $N_{s}$ segments (dismissing the starting vortex) and hydrodynamic pitch angles $\beta_{B V, I, j}^{b_{j}}$ and $\beta_{B V, O, j}^{b_{j}}$, which are distinct and refer to the inner (radially closer to the hub) and outer (radially farther from the hub) trailing vortices, respectively, the total velocity induced $\vec{V}_{H H S V, i j}^{b_{i} b_{j}}$ by a single HHSV $j$ from blade $b_{j}$ at a CP $i$ on blade $b_{i}$ can be computed by the superposition of equation (2.1):

$$
\vec{V}_{H H S V, i j}^{b_{i} b_{j}}=\sum_{k_{j}=1}^{N_{S}} \vec{V}_{V S, i j, k_{j} k_{j+1}}^{b_{i} b_{j}}=\frac{\Gamma_{j}^{b_{j}}}{4 \pi} \vec{v}_{P, i j}^{b_{i} b_{j}}
$$

While in the WLL a fuselage model can be dismissed as connecting the two semispans produces the same effect, in the PLL care must be taken to model the hub, especially because it influences the blades circulation distribution from the root until approximately half span (KERWIN; HADLER, 2010; EPPS, 2017); to do so, an adaptation of Kerwin and Leopold's image vortex was chosen (KERWIN; LEOPOLD, 1964; KERWIN; HADLER, 2010) given the simplicity and accuracy of the results, as the authors demonstrated 
numerically that the model works excellently for vortices that have a sufficiently high pitch angle (usually near the hub), but not as good for vortices with such low angles (generally, elements near the tips, whose influence is small). Other models, such as the one of CAJA (1988), in which he combined the propeller lifting-line representation with a panel method representation for the hub, can be used, although they might be computationally more expensive. The assessment of the use of different hub models can be considered in future implementations.

Thus, assuming a hub radius $R_{h}$, the vertices of each segment of the Image Helical Horseshoe vortex (ImHHSV) $j$ are radially located at $\vec{R}_{I m B V, j}^{b_{j}}$, that depend on the radial positions $\vec{R}_{B V, j}^{b_{j}}$ :

$$
\vec{R}_{I m B V, j}^{b_{j}}=\frac{R_{h}^{2}}{R_{B V, j}{ }^{2}} \vec{R}_{B V, j}^{b_{j}}
$$

Although $\vec{R}$ depends on the CP chordwise location, its absolute value $R$ does not. Moreover, as $\beta_{B V, I, j}^{b_{j}}$ and $\beta_{B V, O, j}^{b_{j}}$ are affected by the CP hydrodynamic pitch angle $\beta_{j}^{b_{j}}$ and its respective $R_{j}$, so are the image vortex hydrodynamic pitch angles $\beta_{\operatorname{Im} B V, I, j}^{b_{j}}$ and $\beta_{I m B V, O, j}^{b_{j}}$; in addition to showing that the wake model of Lerbs (LERBS, 1952) does not agree with the analytic results, Epps (EPPS, 2017) proposed a model that agrees this results and improves the numerical consistency and robustness of the PLL algorithms. So, his wake model is preferred over Lerbs' for the present formulation, and $\beta_{B V, I, j}^{b_{j}}, \beta_{B V, O, j}^{b_{j}}$, $\beta_{I m B V, I, j}^{b_{j}}$, and $\beta_{I m B V, O, j}^{b_{j}}$ are obtained from the following hydrodynamic pitch conservation:

$$
R_{I m B V, j} \tan \left(\beta_{I m B V, \#, j}^{b_{j}}\right)=R_{B V, j} \tan \left(\beta_{B V, \#, j}^{b_{j}}\right)=R_{j} \tan \left(\beta_{j}^{b_{j}}\right) \quad(\#=I, O)
$$

which is why $\beta_{B V, I, i}^{b_{j}} \neq \beta_{B V, O, i}^{b_{j}} \neq \beta_{I m B V, I, j}^{b_{j}} \neq \beta_{I m B V, O, j}^{b_{j}}$. Additionally, the $\mathrm{j}^{\text {th }}$ ImHHSV is assumed to have the same circulation $\Gamma_{j}^{b_{j}}$ of its real counterpart, but with negative value, so the normal velocity at the hub surface is ideally zero. Henceforth, the velocity induced by the ImHHSV of blade $b_{j}$ on $\mathrm{CP} i$ is:

$$
\vec{V}_{I m H H S V, i j}^{b_{i} b_{j}}=\sum_{k_{j}=1}^{N_{S}} \vec{V}_{I m V S, i j, k_{j} k_{j+1}}^{b_{i} b_{j}}=\frac{-\Gamma_{j}^{b_{j}}}{4 \pi} \vec{v}_{I m P, i j}^{b_{i} b_{j}}
$$

$\vec{V}_{I m V S, i j, k_{j} k_{j+1}}^{b_{i} b_{j}}$ has a similar expression to $\vec{V}_{V S, i j, k_{j} k_{j+1}}^{b_{i} b_{j}}$, with the differences that $\vec{r}_{i j, k_{j}}$ is replaced by $\vec{r}_{I m, i j, k_{j}}$ (consequently, $\vec{v}_{P, i j}^{b_{i} b_{j}}$ by $\vec{v}_{I m P, i j}^{b_{i} b_{j}}$ ) and $\Gamma_{j}^{b_{j}}$ by $-\Gamma_{j}^{b_{j}}$. So the inclusion of image vortices does not add unknowns to the circulation problem. Note also that as $R_{h}$ is increased the limiting case of a vortex outside of an infinite wall and its associated image obtained, which makes this image method also appropriate to model half a wing and its fuselage and produce the exact same results as in the complete case. The total velocity 
Figure 23 - A blade section and some of its important parameters. $\beta$ corresponds to the linear formulation, while $\beta_{i}$ to the nonlinear.

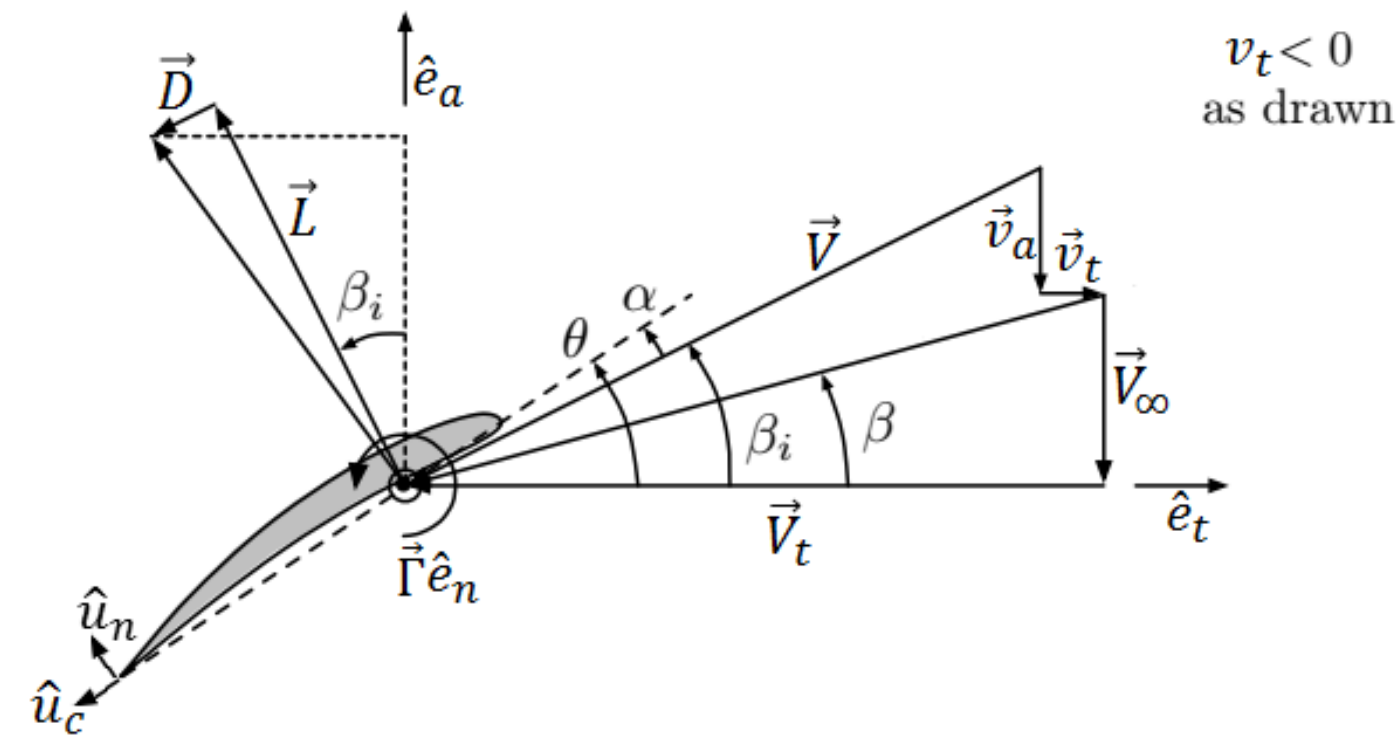

Source: EPPS, 2017 (Adapted).

at $i$ of $b_{i}$ is the superposition of the $N \times Z$ HHSV induced, the $N \times Z$ ImHHSV induced, the advance $\vec{V}_{\infty, i}^{b_{i}}$, and the tangential $\vec{V}_{t, i}^{b_{i}}$ velocities:

$$
\begin{gathered}
\vec{V}_{P, i}^{b_{i}}=\vec{V}_{\infty, i}^{b_{i}}+\vec{V}_{t, i}^{b_{i}}+\sum_{b_{j}=1}^{Z} \sum_{j=1}^{N}\left(\vec{V}_{H H S V, i j}^{b_{i} b_{j}}+\vec{V}_{I m H H S V, i j}^{b_{i} b_{j}}\right) \\
\vec{V}_{t, i}^{b_{i}}=\vec{\omega} \times \vec{R}_{i}^{b_{i}}
\end{gathered}
$$

Applying the PBC at this CP (PISTOLESI, 1937), whose unit vector is $\hat{u}_{n, i}^{b_{i}}$, again establishes zero normal velocity component:

$$
\begin{aligned}
\hat{u}_{n, i}^{b_{i}} \cdot \vec{V}_{P, i}^{b_{i}}= & 0 \rightarrow \hat{u}_{n, i}^{b_{i}} \cdot\left(\vec{V}_{\infty, i}^{b_{i}}+\vec{V}_{t, i}^{b_{i}}\right)+\sum_{b_{j}=1}^{Z} \sum_{j=1}^{N} \hat{u}_{n, i}^{b_{i}} \cdot\left(\vec{V}_{H H S V, i j}^{b_{i} b_{j}}+\vec{V}_{I m H H S V, i j}^{b_{i} b_{j}}\right)=0 \rightarrow \\
& \sum_{b_{j}=1}^{Z} \sum_{j=1}^{N} \hat{u}_{n, i}^{b_{i}} \cdot\left(\vec{V}_{H H S V, i j}^{b_{i} b_{j}}+\vec{V}_{I m H H S V, i j}^{b_{i} b_{j}}\right)=-\hat{u}_{n, i}^{b_{i}} \cdot\left(\vec{V}_{\infty, i}^{b_{i}}+\vec{V}_{t, i}^{b_{i}}\right)
\end{aligned}
$$

Again, equation (2.2) can as well be more conveniently written using equations (2.18) and (2.21): 


$$
\begin{gathered}
\sum_{b_{j}=1}^{Z} \sum_{j=1}^{N} m_{i j}^{b_{i} b_{j}} \Gamma_{j}^{b_{j}}=-4 \pi \times W_{\infty, i}^{b_{i}} \\
m_{i j}^{b_{i} b_{j}}=\hat{u}_{n, i}^{b_{i}} \cdot\left(\vec{v}_{P, i j}^{b_{i} b_{j}}-\vec{v}_{I m P, i j}^{b_{i} b_{j}}\right) \\
W_{\infty, i}^{b_{i}}=\hat{u}_{n, i}^{b_{i}} \cdot\left(\vec{V}_{\infty, i}^{b_{i}}+\vec{V}_{t, i}^{b_{i}}\right)
\end{gathered}
$$

Equation (2.25) is imposed for the $N \times Z$ corresponding control points, which results this time in a system of $(N \times Z) \times(N \times Z)$ equations that can similarly be written in a matrix form, according to equation $(2.7)$ :

$$
\mathbb{M}_{P} \boldsymbol{\Gamma}_{P}=-\mathbf{W}_{\infty P}
$$

whose elements are $m_{i j}^{b_{i} b_{j}}$. Note that $\mathbb{M}_{P}$ consists of influence sub-matrices $\mathbb{M}^{b_{i} b_{j}}$ that account for the influence of blade $b_{j}$ on blade $b_{i}$ :

$$
\mathbb{M}_{P}=\left[\begin{array}{ccc}
\mathbb{M}^{11} & \cdots & \mathbb{M}^{1 Z} \\
\vdots & \mathbb{M}^{b_{i} b_{j}} & \vdots \\
\mathbb{M}^{Z 1} & \cdots & \mathbb{M}^{Z Z}
\end{array}\right]
$$

Likewise, the circulation and freestream arrays are assembled from the blades 'sub-arrays'. For $\boldsymbol{\Gamma}_{P}$ and $\mathbf{W}_{\infty P}$ :

$$
\boldsymbol{\Gamma}_{P}=\left[\begin{array}{c}
\Gamma^{1} \\
\Gamma^{2} \\
\vdots \\
\Gamma^{Z}
\end{array}\right], \mathbf{W}_{\infty P}=\left[\begin{array}{c}
\mathbf{W}_{\infty}^{1} \\
\mathbf{W}_{\infty}^{2} \\
\vdots \\
\mathbf{W}_{\infty}^{N}
\end{array}\right]
$$

\subsubsection{Linear scheme}

The linear estimate for the PLL problem assumes that the CP hydrodynamic pitch angle $\beta_{j}^{b_{j}}$ is not affected by the induced velocities (figure 23), and in a design framework $\beta_{j}^{b_{j}}$ is obtained from equation (2.29), with $\hat{e}_{t, i}$ and $\hat{e}_{a, i}$ the unit vectors in the tangential and axial directions of the propeller reference frame, but mapped into the $i^{\text {th }}$ blade section cylindrical surface:

$$
\beta_{i}^{b_{i}}=\tan ^{-1}\left(\frac{\vec{V}_{P, i}^{b_{i}} \cdot \hat{e}_{a, i}}{\vec{V}_{P, i}^{b_{i}} \cdot \hat{e}_{t, i}}\right)
$$

Note that such mapping is necessary for both $\vec{e}_{a, i}$ and $\vec{V}_{t, i}^{b_{i}}$ point into different directions for each CP (as the CP line is not straight as in the classic approach). Therefore, 
the 'linear' $\beta_{L, i}^{b_{i}}$ is obtained for $\vec{V}_{H H S V, i j}^{b_{i} b_{j}}=\vec{V}_{I m H H S V, i j}^{b_{i} b_{j}}=0$. With the wake geometry defined, the circulation distribution for each blade can be obtained simultaneously by solving equation (2.26), and the hydrodynamic forces are calculated again considering only the trailing vortices induced velocities:

$$
\begin{gathered}
\delta \vec{F}_{P, i}^{b_{i}}=\rho \Gamma_{i}^{b_{i}} \vec{V}_{T V P, i}^{b_{i}} \times \delta l_{i}^{b_{i}} \rightarrow \\
\delta F_{P, i}^{b_{i}}=\rho \Gamma_{i}^{b_{i}} V_{T V P, i}^{b_{i}} \delta l_{i}^{b_{i}} \sin \psi_{i}^{b_{i}} \\
\vec{V}_{T V P, i}^{b_{i}}=\vec{V}_{\infty, i}^{b_{i}}+\vec{V}_{t, i}^{b_{i}}+\sum_{b_{j}=1}^{Z} \sum_{j=1}^{N}\left(\vec{V}_{H H S V, i j}^{b_{i} b_{j}}+\vec{V}_{I m H H S V, i j}^{b_{i} b_{j}}-\vec{V}_{V S, i j, \frac{N_{s}+1}{2} \frac{N_{s}+2}{2}}^{b_{i} b_{j}}-\vec{V}_{I m V S, i j, \frac{N_{s}+1}{2} \frac{N_{s}+2}{2}}^{b_{i} b_{j}}\right)
\end{gathered}
$$

Prandtl's hypothesis is again assumed to be true along with the three-dimensional version of the KJT, and the potential hydrodynamic normal section force coefficients for each blade $C_{n P P o t, i}^{b_{i}}$ can be calculated.

$$
C_{n P P o t, i}^{b_{i}}=\frac{\delta F_{P, i}^{b_{i}}}{\frac{1}{2} \rho V_{P, i}^{b_{i}{ }^{2} c_{i}} \delta l_{i}}
$$

\subsubsection{Nonlinear scheme}

In addition to approximating the potential and viscous estimates for the normal hydrodynamic force coefficients, the nonlinear scheme also corrects the hydrodynamic pitch distributions by the induced velocities; with an estimate for $C_{n P P o t, i}^{b_{i}}$, the effective AoA can be calculated assuming the linear relation of equation (2.11) for each section of each of the $Z$ blades, and then the viscous counterparts $C_{n P V i s, i}^{b_{i}}$ and $\frac{\partial C_{n, i}}{\partial \alpha_{i}}$ Vis are obtained as a functions of $\alpha_{e f f, i}^{b_{i}}, \alpha_{L 0, i}$, and $R e_{i}^{b_{i}}$, as in equations (2.12) and (2.14).

Later, the nonlinear hydrodynamic pitch angle $\beta_{N, j}^{b_{j}}$ is calculated and used to correct the wake geometry through equation (2.20); equation (2.29) is unfeasible for calculating $\beta_{N, j}^{b_{j}}$ in the current formulation as the blades cylindrical mapping suitably changes the resulting induced velocities in the nonstraight $\frac{3 c}{4} \mathrm{CP}$ line in comparison to the classic $\frac{c}{2}$, so a simpler and more consistent angle compatibility equation that satisfies the basic angle correspondence among the geometric pitch $\theta_{i}, \alpha_{e f f, i}^{b_{i}}$, and $\beta_{i}^{b_{i}}$ is used instead:

$$
\beta_{i}^{b_{i}}=\alpha_{e f f, i}^{b_{i}}-\theta_{i}
$$

Note that regardless of the differences between $C_{n P V i s, i}^{b_{i}}$ and $C_{n P P o t, i}^{b_{i}}$ of the first iteration, the nonlinear scheme is necessary to correct the initial wake geometry, and only after obtaining an updated value for $\boldsymbol{\Gamma}_{P}$, the coefficients are used as a stopping criterion, which, as in the WLL, if either the absolute or relative differences for each blade are not within a desirable tolerance, each $\frac{\partial C_{n_{i}}}{\partial \alpha_{i}}$ is updated according to equation (2.14). 


\subsubsection{Post-processing and flowchart}

In addition to the hydrodynamic forces that are generated by the blades, which must be decomposed into the propeller thrust and torque, the vortex superposition that models the hub contributes to a pressure $\operatorname{drag} D_{P}$ that is computed by the model proposed by WANG (1985):

$$
D_{P}=\frac{\rho}{16 \pi}\left(\log \frac{R_{h}}{R_{0}}+3\right)\left(N_{B} \Gamma_{h}\right)^{2}
$$

This model assumes that all the vortices inside the hub are represented by a Rankine vortex with a viscous core radius $R_{0}$ that must be estimated, but variations from its precise value are not significant as $D_{P}$ depends on a logarithm of this quantity. Additionally, while in the open-water case, the circulation strength $\Gamma_{h}$ is the same regardless of the blade, for nonuniform flows, $\Gamma_{h}$ may vary from blade to blade; thus, the highest of these $Z$ values are considered. Finally, considering the influence of the pressure drag, the thrust and torque coefficients can be written as:

$$
\begin{aligned}
& K_{T}=\frac{1}{\rho n^{2} D^{4}}\left\{\frac{\rho}{2} \sum_{b_{i}=1}^{Z} \sum_{i=1}^{N} V_{P, i}^{b_{i}}{ }^{2} \delta A_{i}\left[C_{n, i}^{b_{i}} \cos \left(\beta_{N, i}^{b_{i}}\right)-C_{c, i}^{b_{i}} \sin \left(\beta_{N, i}^{b_{i}}\right)\right]-D_{P}\right\} \\
& K_{Q}=\frac{1}{2 n^{2} D^{5}} \sum_{b_{i}=1}^{Z} \sum_{i=1}^{N} V_{P, i}^{b_{i}}{ }^{2} R_{i}^{b_{i}} \delta A_{i}\left[C_{n, i}^{b_{i}} \sin \left(\beta_{N, i}^{b_{i}}\right)+C_{c, i}^{b_{i}} \cos \left(\beta_{N, i}^{b_{i}}\right)\right]
\end{aligned}
$$

$n$ is the rotation rate and for inviscid cases $C_{c}=0$, i.e., only the induced torque $K_{Q i}$ is calculated. The presence of section viscous drag increases the overall torque while it decreases the thrust; thus, its inclusion should increase the accuracy of the hydrodynamic forces and moments, if the flow regime is adequate.

The following flowchart illustrates the PLL pipeline: 
Figure 24 - Flowchart of the WLL formulation

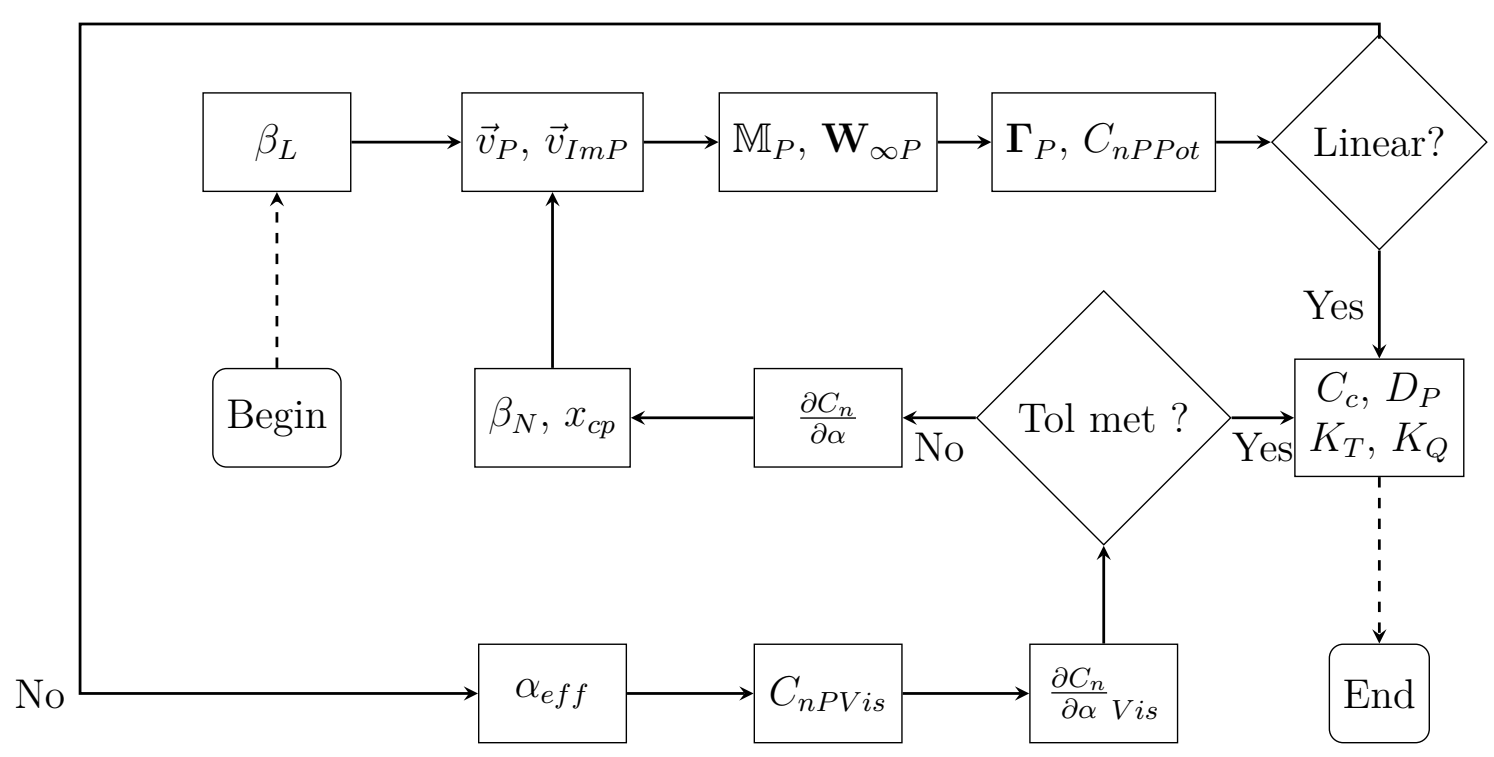

Source: Author

\subsection{Source of 2-D data on the lifting-line}

A common practice on lifting-line methods is some assumption about the $C_{n}$ and $C_{c}$ data that comprises the wing/blade section distributions, and the most common one derives from the results of the TFT (KATZ; PLOTKIN, 2001), which establishes $C_{n}=2 \pi\left(\alpha_{e f f}-\alpha_{L 0}\right)$ and $C_{c}=0$ for a foil having zero-lift angle of attack $\alpha_{L 0}$. However, undoubtedly the more representative is the information about the curve slopes and the zero-lift angles of attack, the more accurate should be the section force and moment distributions - which directly influences the overall forces and moments. So, other options to the TFT are the use of either numerical or experimental results to improve the estimates for $C_{n}$ and $C_{c}$; one could, for instance, simulate or experiment families of foils for a variety of angles of attack and Reynolds numbers of interest to generate a database that can be used on the LLT, and specifically for the simulations, the generation of a database beforehand is computationally more efficient than performing them on-the-fly. In spite of the source, either experiments or simulations must be carefully performed to (i) reproduce the local flow conditions in order to have accurate estimates for the force coefficients and (ii) keep the uncertainties to the minimum as they will contribute to the overall uncertainty of the method.

To exemplify this influence, a comparison among these three sources of data was carried out for the NACA 66 (TMB Modified Nose and Tail) thickness with a = 0.8 camberline family. This family was chosen because it has been experimentally wellcharacterized (BROCKETT, 1966) and widely used in many propellers (DENNY, 1968; 
GHOSE, 2004). Thus, in addition to comparing the values of $C_{n}$ and $C_{c}$ from the several methods, they will be used in the PLL so the dependence of $K_{T}$ and $K_{Q}$ on them can also be assessed. A typical NACA66 section is presented on figure 25:

Figure 25 - Example of NACA 66 (TMB Modified Nose and Tail) a $=0.8$ section. $\frac{t_{0}}{c}=$ 0.0679 and $\frac{f_{0}}{c}=0.0501$

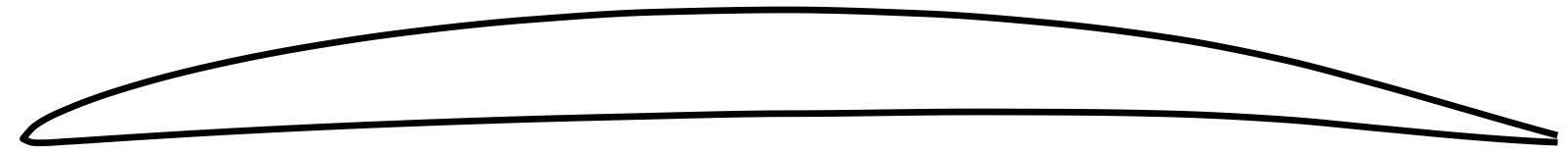

Source: Author

For this foil family, experiments have been conducted by BROCKETT (1966), leading to an empirical $C_{n}$ expression that works adequately for relatively thin lowcambered foils and $R e \geq 6 \times 10^{6}$, in terms of the foil maximum thickness-to-chord ratio $\frac{t_{0}}{c}$ and maximum camber-to-chord ratio $\frac{f_{0}}{c}$ :

$$
\begin{gathered}
C_{n}=2 \pi\left(1-0.83 \frac{t_{0}}{c}\right)\left(\alpha+2.05 \frac{f_{0}}{c}\right) \\
C_{c}=0.0085
\end{gathered}
$$

$C_{c}$ is typically assumed 0.0085 in spite of the other variables (DENNY, 1968; EPPS et al., 2009); note, also, that no uncertainty - or confidence interval - is given for such expressions. For the TFT, as mentioned, the expressions for $C_{n}$ and $C_{c}$ are:

$$
\begin{gathered}
C_{n}=2 \pi\left(\alpha+2 \frac{f_{0}}{c}\right) \\
C_{c}=0
\end{gathered}
$$

In order to build a numerical database, simulations have been carried out using Star-CCM+ commercial software: the foil geometries were drawn based on tabulated values for standard thickness and meanline distributions (CARLTON, 2019) in a domain of approximately 200,000 elements in a trimmed mesh configuration - figure 26 -, with five chord lengths fore, 20 chord lengths aft, and a total height of nine chord lengths; the wake refinement had a spread angle of $8^{\circ}$ and five chord lengths, with cells growing in a slow fashion. A steady-state $K-\omega$ Shear Stress Transport (SST) model with low Reynolds damping was used, because the ranges of blade section Reynolds numbers for the model-scale propellers of interest were in between the order of $1 \times 10^{4}$ and $1 \times 10^{5}$, so to simplify the database, it was assumed that the dependence on $R e$ was negligible and an averaged value for this parameter was chosen for each foil. The boundary conditions were 
velocity inlet for the inlet, upper, and lower parts, pressure for the outlet, and wall for the foil. Additionally, the elements near the foil section were kept sufficiently small so the wall $y+$ values were no higher than 1 aside near the stagnation points. The convergence criteria were asymptotic for $C_{n}$ and $C_{c}$ within a range of 1000 iterations, with maximum differences no higher than $1 \times 10^{-4}$ and $1 \times 10^{-5}$, respectively. The Sdr criterion was set to reach a minimum value of $1 \times 10^{-5}$. For some simulations, however, the established criteria could not be satisfied due to a combination of the foil geometry, angle of attack, and inflow velocity; in such cases, the steady-state was changed for an unsteady model in order to add numerical diffusion, and when convergence was still not achieved, period-based-average values of the coefficients were calculated. For the sake of its use on the present formulations, the database was built considering the sections throughout the blade for each propeller used for validation and linear interpolations of $C_{n}$ and $C_{c}$ as functions of $\frac{t}{c}$ were used to retrieve the coefficients in any radial location of the blade, as $\frac{t}{c}$ is a function of this location; in terms of $\alpha$, the coefficients were both linearly interpolated and extrapolated. Of course, blade section hydrodynamic characteristics change with maximum camber and thickness, as well as with the angle of attack and Reynolds number, but more careful analyses span to a separate study and are out of the scope of this work.

Finally, mesh convergence studies (i.e., solution verification) were performed for two foil sections (a NACA 0012 and one from the NACA 66 a $=0.8$ family), so an idea about the order of magnitude for the uncertainties due to the use of Star-CCM+ as a source of 2-D data can be given, at least. Of course, depending on the flow regime, these uncertainties are underestimated - especially for thick foils at relatively high angles of incidence, in which strong separation bubbles are present; this, however, might be the best approach to quantify the uncertainties in the aerodynamic coefficients. To perform this uncertainty estimation, the grid described in the above paragraph was refined and coarsened twice - each time by a ratio of $\sqrt{2}$-, so families of five unstructured grids were obtained; the uncertainties, as well as the rates of convergence and extrapolated values, were obtained with the help of the opensource tool named Numerical Uncertainty Analysis (ECA; HOEKSTRA, 2014; VAZ; ECA; TOXOPEUS, 2018), from the Maritime Institute Netherlands (MARIN), and part of the theory associated will be described on the next chapter, devoted for this purpose. For now, figures 27 and 28 present the uncertainties for the two foils.

The two figures show that converge is rather difficult, which is likely due to several factors, as the complexity of the equations (turbulent models) and lack of geometrical similarity of the grids - the latter hardly obtainable by the use of unstructured grids (ECA; HOEKSTRA, 2014). As such, scatter data is usually frequent in those cases, followed by relatively large uncertainties. However, the uncertainties produced are still useful, for their values reflect such trends, and also because the standard deviation of the fits is lower than the data range parameter, whose appropriate explanation will be given in the next chapter. 
Figure 26 - Star-CCM+ CFD domain for the NACA 66 (TMB Modified Nose and Tail) a $=0.8$ section simulations

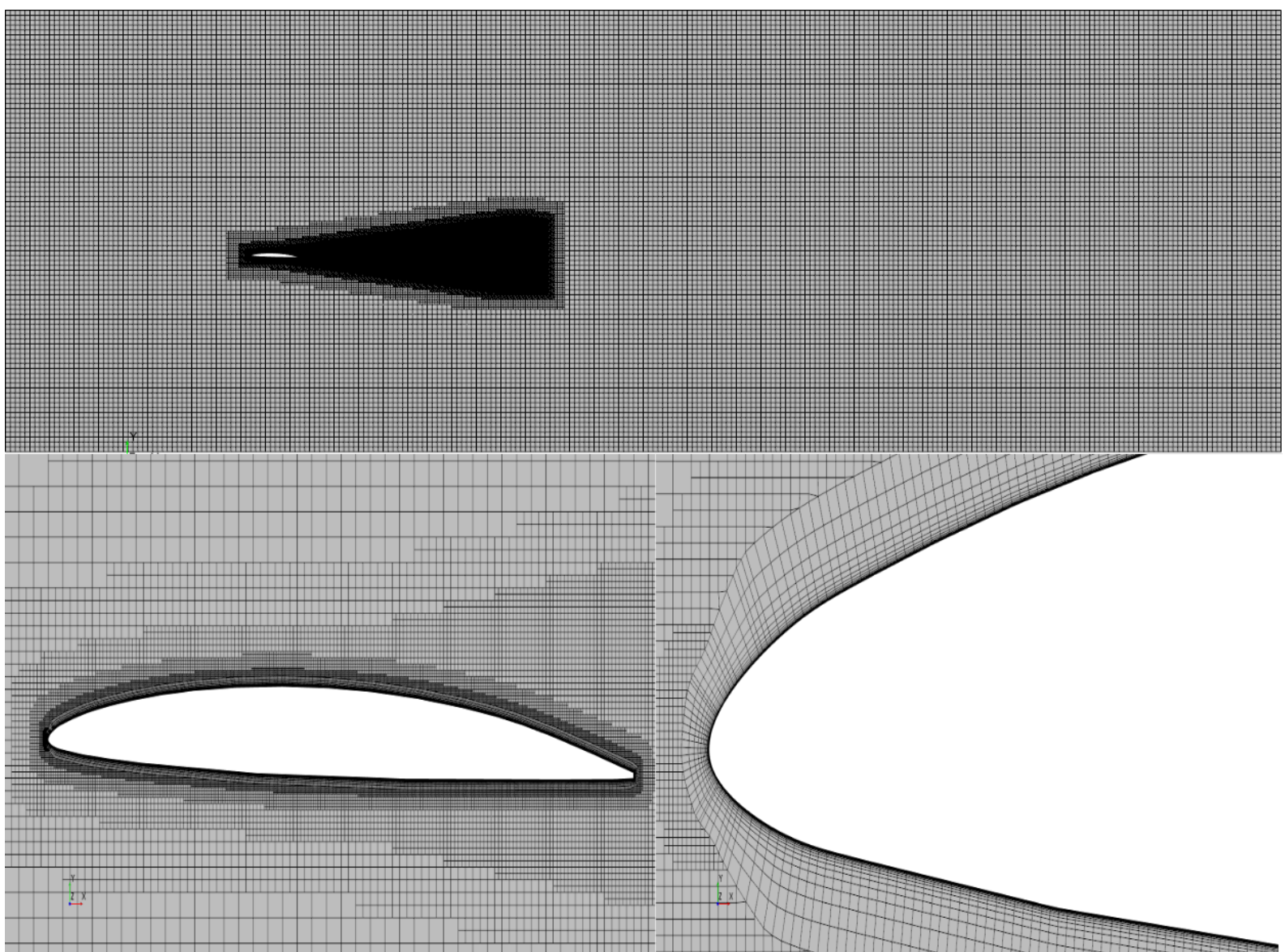

Source: Author

For the described mesh (results for $1.5<\frac{h}{h_{f}}<2.0$ ), the uncertainties obtained are in the order of $u_{C_{n}}=1 \times 10^{-2}$ and $u_{C_{c}}=1 \times 10^{-4}$, for $C_{n}$ an $C_{c}$, respectively.

Figure 29 presents a visual comparison of the magnitude of the velocity fields - normalized by the freestream - between two NACA 66 foils with different values for maximum thickness and camber: the thinnest has $\frac{t_{0}}{c}=0.0933$ and $\frac{f_{0}}{c}=0.0193$ - whose uncertainty has bee presented on figure 28 -, while the thickest has $\frac{t_{0}}{c}=0.1449$ and $\frac{f_{0}}{c}=0.0426$. For the thinnest foil, there is a relatively low region of flow detachment near the trailing edge, a thin wake region, and the flow seems well behaved. For the thickest one, on the other hand, the separation region as well as its wake are larger, meaning that flow instabilities are likely to happen sooner.

Figures 30 and 31 present the comparison among the three sources for two NACA 66 sections. For the thinnest foil, the normal force curves evidence reasonable agreement for most of the $\alpha$ range, with the differences increased with the increase of the AoA; such trend corroborates the well-behaved flow observed on figure 29, which explains why $C_{n}$ is reasonably characterized by both theory and experimental expressions: in terms 
Figure 27 - Uncertainties of $C_{n}$ and $C_{c}$, calculated with Star-CCM+, as function of the refinement ratio $h$. NACA 0012.
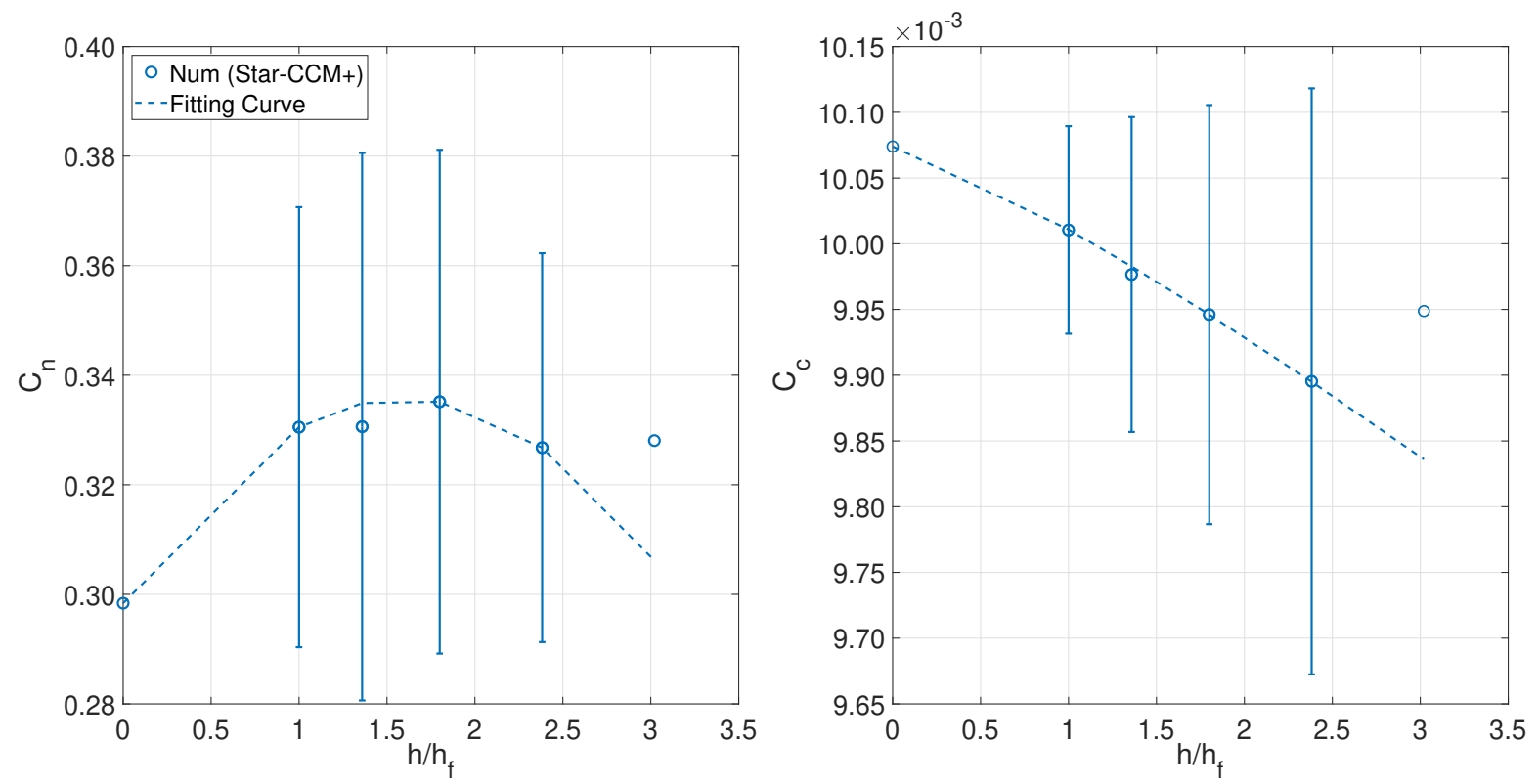

Source: Author

Figure 28 - Uncertainties of $C_{n}$ and $C_{c}$, calculated with Star-CCM+, as function of the refinement ratio $h$. NACA 66 TMB Modified $\mathrm{a}=0.8-\frac{t_{0}}{c}=0.0933, \frac{f_{0}}{c}=0.0193$.
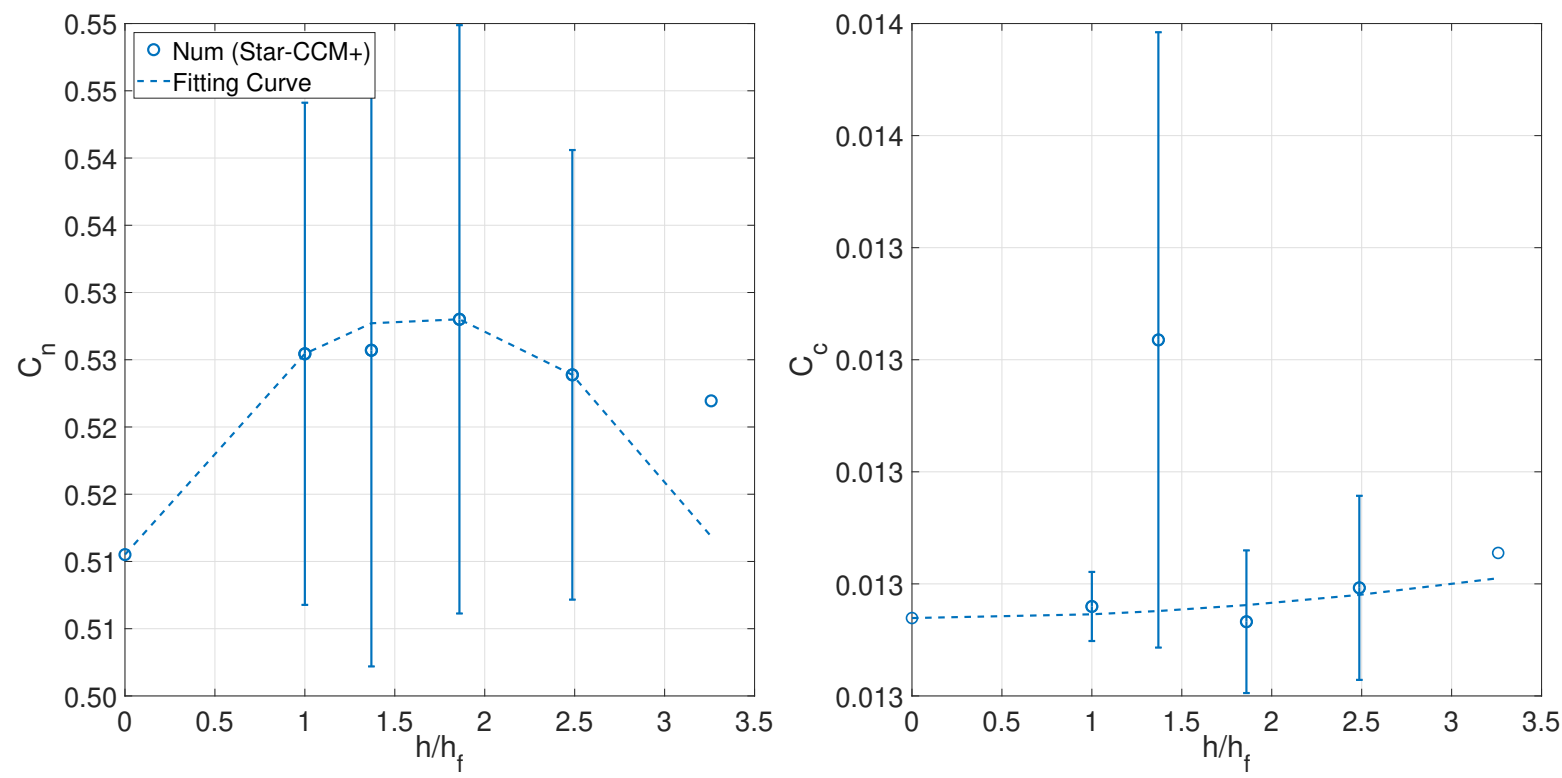

Source: Author

of normal force, the use of any of these curves would lead to small differences on the overall coefficients. For $C_{c}$, on the other hand, the differences from one another are much more significant. For the thickest foil, however, the differences among the three curves are more visible: in terms of normal force, the theoretical curve presents the highest of 
Figure 29 - Magnitudes of the velocity fields normalized by the freestream velocity thinnest (upper) and thickest (lower) foils.

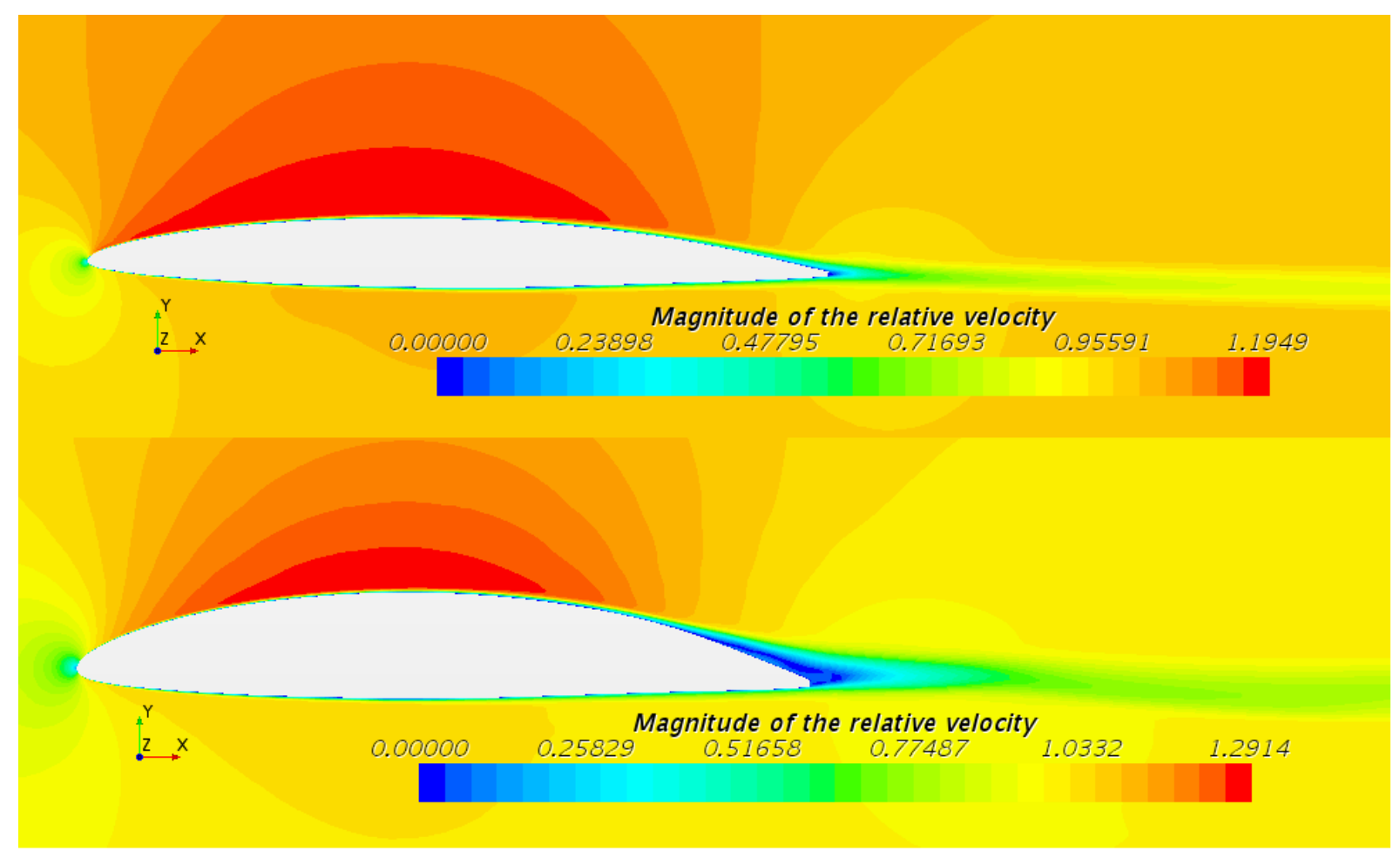

Source: Author

all slopes, followed by the experimental expression. The zero-lift angle of attack also changes significantly from numerical to the other two, which are nearly the same. The flow visualization of figure 29 qualitatively explains the large differences for this foil and why it cannot be adequately modeled at least by the theoretical expressions; it is evident that the choice of the 2-D data would strongly influence the outcomes for the LLT, and better results would probably be obtained by using either numerical or experimental sources. In terms of $C_{c}$, the trend is maintained, but more accentuated.

Cavitation inception is another trend that can be included through the source of 2-D data; if information about the minimum pressure coefficient $C_{P, \min }$ as function of the angle of attack is stored in the database - along with its chordwise location - a distribution of $C_{P, \min }$ along each blade could be retrieved and compared to the vapor pressure; when any local pressure is minimum, then it is likely that cavitation will occur in that region. Certainly, this is an estimate that as well as for viscosity, does not consider three-dimensional effects. A similar approach was developed by BROCKETT (1966), in which minimum pressure envelopes, parameterized by thickness-to-chord and camber-tochord ratios, were computed for families of foils and design charts were drawn as a function of the angles of attack. 
Figure 30 - Comparison of $C_{n}$ and $C_{c}$ as function of $\alpha$ for several sources of 2-D data . NACA 66 TMB Modified $\mathrm{a}=0.8-\frac{t_{0}}{c}=0.0933, \frac{f_{0}}{c}=0.0193$.
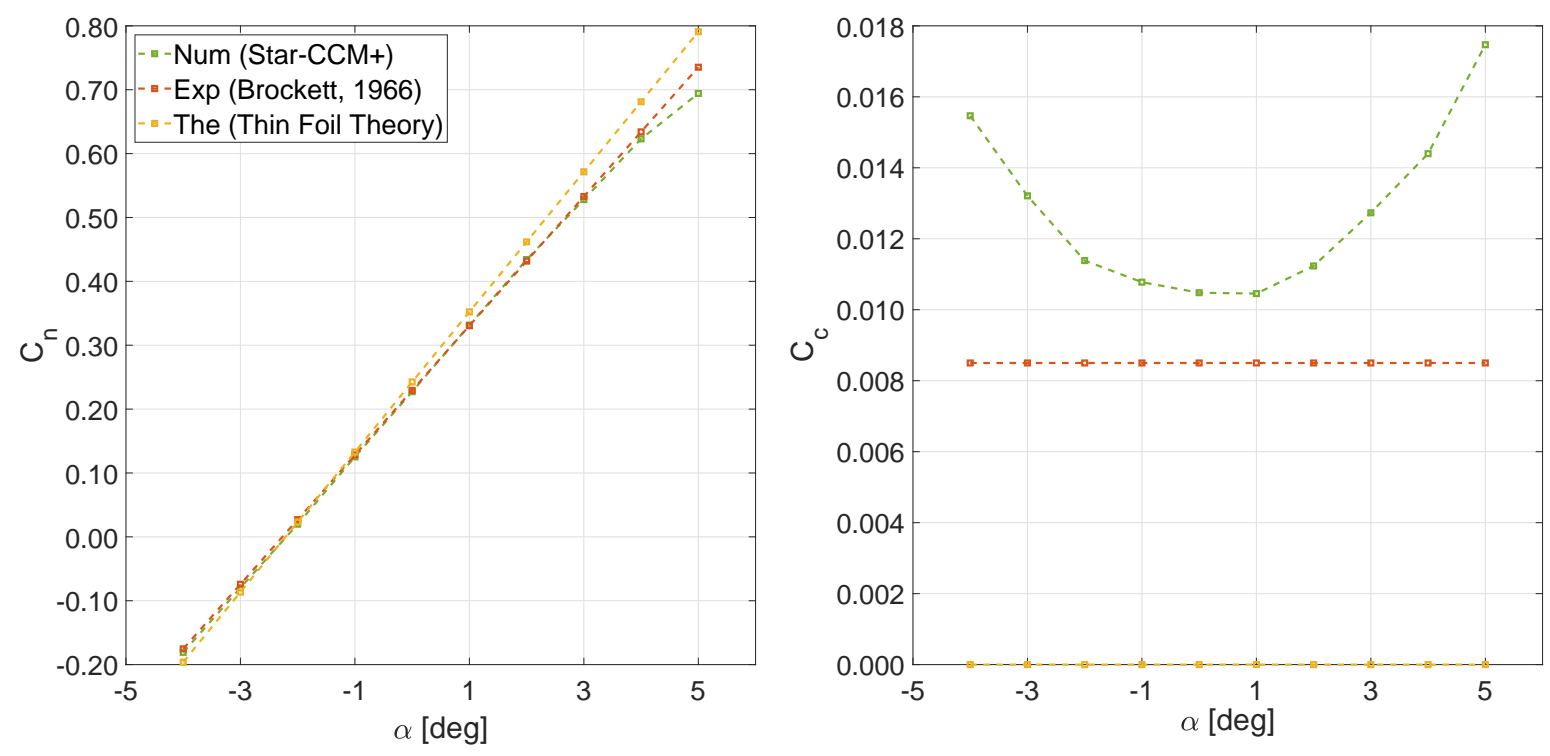

Source: Author

Figure 31 - Comparison of $C_{n}$ and $C_{c}$ as function of $\alpha$ for several sources of 2-D data. NACA 66 TMB Modified $\mathrm{a}=0.8-\frac{t_{0}}{c}=0.1449, \frac{f_{0}}{c}=0.0426$.
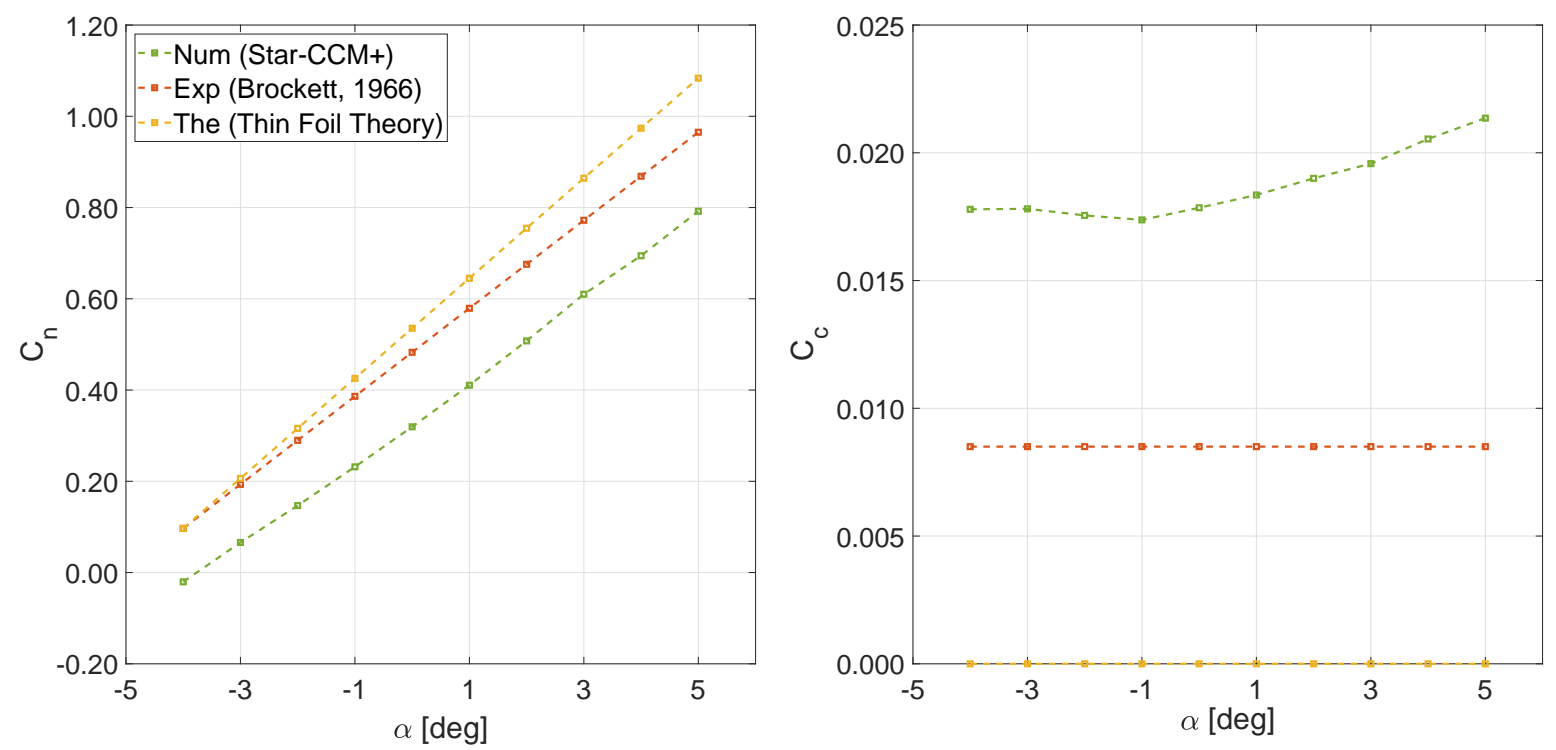

Source: Author 


\section{Verification \& Validation}

To assess the reliability of the proposed lifting-line formulations, verification and validation procedures were performed as recommended by the American Society of Mechanical Engineers (ASME); according to the ASME $20^{\text {th }}$ Verification and Validation (VV) Committee (ASME, 2009), the concern of VV is the assessment of the accuracy of a computational simulation, which is ultimately accomplished by validation, defined as the process of determining the degree to which a proposed model accurately represents the real world from its perspective. Validation, however, must be preceded by code and solution verifications; while verification is the establishment of numerical accuracy independently of physical accuracy, code verification is necessary to ensure that the code developed is free of errors, and solution verification estimates the numerical accuracy of a particular solution.

Validation estimates a range within which the simulation modeling error lies, which is accomplished by comparison between simulation and experimental results, both appropriately specified for desired variables and conditions. Thus, it can be inferred that (i) there is no validation without experimental data for comparison and (ii) the validation must cover a range of conditions within a domain of interest.

\subsection{Method}

Denoting $T$ as a true, but unknown, value of a certain variable used for validation, the predicted value of $T$ from the simulation solution as $S$, and the value determined from experiments as $D$, the validation comparison error $E$ is defined as:

$$
E=S-D
$$

The error $\epsilon_{s}$ between $S$ and $T$ is simply their difference:

$$
\epsilon_{S}=S-T
$$

Similarly, for the experimental value:

$$
\epsilon_{D}=D-T
$$


Such that equation (3.1) can be rewritten to conclude that the error is the combination of all of the numerical and experimental errors:

$$
E=\epsilon_{S}-\epsilon_{D}
$$

The errors arising from the numerical solution can be categorized as (i) input error $\epsilon_{i}$-due to errors in the input parameters, (ii) modeling error $\epsilon_{m}$-due to assumptions and approximations, and (iii) numerical error $\epsilon_{n}$ - due to the numerical solutions, such that:

$$
\epsilon_{S}=\epsilon_{m}+\epsilon_{n}+\epsilon_{i}
$$

Estimation of $\epsilon_{m}$, to within an uncertainty range, is the purpose of the validation. Thus, combining equations (3.4) and (3.5), and rearranging the terms:

$$
\epsilon_{m}=E-\left(\epsilon_{n}+\epsilon_{i}-\epsilon_{D}\right)
$$

The standard uncertainties that correspond to the aforementioned errors are $u_{d}$, $u_{i}$, and $u_{n}$, and the validation standard uncertainty $u_{v}$ is the estimate of the standard deviation of the parent distribution of the combined errors $\left(\epsilon_{n}+\epsilon_{i}-\epsilon_{d}\right)$. Thus:

$$
\epsilon_{m} \in\left[E-u_{v}, E+u_{v}\right]
$$

Moreover, if the three errors are independent, then:

$$
u_{v}=\sqrt{u_{n}^{2}+u_{i}^{2}+u_{d}^{2}}
$$

Such an assumption holds reasonable when the validation variable is directly measured. On the other hand, if the validation variable is determined by a data reduction equation, then it can be a function of primary, shared variables and, consequently, the errors do not become independent.

\subsection{Code Verification and Solution Verification}

The objective of verification is the establishment of numerical accuracy, so code verification and solution verification are procedures to estimate $u_{n}$. Code verification is distinct from solution verification in the sense that the former is the procedure of evaluating the mathematical correctness of the developed code, and it involves evaluation of the errors from known benchmarks. Solution verification, on the other hand, is the process of estimating the numerical uncertainties of a numerical solution, but not necessarily 
comparing them to known benchmarks. Code verification precedes solution verification, but both have systematic grid refinement as their basis.

Code verification is done by systematic discretization and solution convergence monitoring towards a known benchmark, whose best option is an exact analytic solution that is sufficiently complex for all the terms in the governing equations to be exercised; therefore, there exists a perception that general accuracy verification of codes for complex problems is not feasible due to the lack of adequate analytic solutions available. However, since the achievement of a convincing code verification depends on a complete test of the code relevant features, specialists on the topic suggest methods to circumvent this limitation. One of these methods is the use of the Method of Manufactured Solutions (MMS), which has been successfully demonstrated in many codes and applies to ones based on the solution of partial differential or integrodifferential equations. Another option is code-tocode comparison, in which the idea is to use a previously verified code as benchmark; this approach can be done at two levels: by (i) requiring that both codes have the same interior and boundary discretizations and close to machine-zero convergence, or (ii) high-resolution solutions that are considered as good approximations to exact solutions. The former is effective when the code is a new version of a previously verified code and the new version does not change any of the discretizations, but beyond this, this option is not sufficiently convincing. The latter is an option if the benchmark has been thoroughly verified and if the solutions have resolved all the important length scales of the problem. However, MMS is preferred over code-to-code comparisons for its formalism.

With a nontrivial exact analytic solution available, code can be verified by observation of the solution convergence and its direct comparison with the expected values. Convergence is generally assessed with the aid of a discretization variable $h$, i.e., a measure of the level of refinement, which can be a typical length in space and a time-step in finite-difference or finite-volume codes, an element size in finite-element codes, or the inverse of the number of vortices in a discrete-vortex method. Within this context, an order of convergence $p$ can be defined based on the behavior of the error of the numerical solution, and the discretization error $E$ can have various definitions, but the most common refers to either the absolute or the relative difference between the continuum $f_{\text {the }}$ and the discrete $f(h)$ values:

$$
\begin{aligned}
& E_{a}=\left|f_{\text {the }}-f(h)\right| \\
& E_{r}=\left|\frac{f_{\text {the }}-f(h)}{f_{\text {the }}}\right|
\end{aligned}
$$

For a well-behaved problem, the difference $f_{\text {the }}-f(h)$ is proportional to $h^{p}$ in the asymptotic limit of $h \rightarrow 0$, such that: 


$$
\begin{gathered}
E_{a}=C h^{p}+H \cdot O \cdot T_{a} \\
E_{r}=\left|\frac{C h^{p}}{f_{\text {the }}}\right|+H \cdot O \cdot T_{\cdot r}
\end{gathered}
$$

in which H.O.T. are high order terms. For a meaningful assessment of $p$, grid refinement is required with a minimum ratio of 1.3 for successive grids. The values of $\frac{E_{a}}{h^{p}}$ should become constant as the grid is refined. There is an expected theoretical $p_{\text {the }}$ that might differ from the observed $p$. A significant difference indicates at least one of the possible issues:

- the grid convergence has not been carried to a sufficient refinement level;

- there are more significant errors than solely due to discretization, leading to detailed code review;

- boundary conditions may not be appropriate;

- initial conditions may not be appropriate;

- incomplete iterative convergence and round-off errors.

Solution verification follows code verification, and the most widely used method for error estimation is the classical Richardson Extrapolation (RE) - which has been extensively studied. Sometimes, for relatively difficult problems, generalized RE and Least Square methods are used in place. Error and uncertainty estimates, although related, are not the same, as an error estimate is intended to provide an improvement to the result of a calculation, and an uncertainty estimate is intended to provide an interval around this result such that the true value falls within a certain probability. The quantification of this probability is addressed by the uncertainty estimation, and a common percentage is approximately 95\%. This confidence level is compatible with the plus-or-minus one standard deviation around the average for a Gaussian distribution, although there will be no assumptions about the distribution of error. The uncertainty estimate $u_{n}$ can be calculated by a Grid Convergence Index (GCI), obtained through procedures that ensure the percentage aforementioned.

To perform solution verification, one of the requirements is that iterative convergence must be achieved for all simulations, otherwise, the estimated uncertainty is polluted by incomplete iteration errors; the iterative convergence is commonly assumed to be achieved when the normalized errors decrease at least three orders of magnitude for each equation solved over the entire domain, although this assumption has not been convincingly demonstrated. Especially for time-dependent simulations, the iterative convergence at every time-step must be ensured, and despite the time-dependency, it is preferred to 
reduced such iterative error to a negligible level in comparison to the discretization. If the uncertainty associated with the iteration error is much lower than the uncertainty $u_{h}$ associated with the discretization error, then:

$$
u_{n}=u_{h}
$$

One of the approaches for $u_{h}$ estimation is through a five-step procedure that starts with the definition of $h$, which, in particular, is a characteristic horseshoe vortex length; for most of the cases, this particular length is the average of the bound vortex length:

$$
h=\frac{\sum_{i=1}^{N} \delta l_{i}}{N}
$$

At least three significantly different sets of grids must be chosen with systematic refinement from one another, such that successive grids should differ by each other by a ratio of at least 1.3 in the number of elements, a value based on experience. Near an assumed convergence region whose error monotonically decreases with increasing refinement, $f(h)$ can be written with the aid of equations (3.9) and (3.11):

$$
f(h)=f_{e x t}+C h^{p}
$$

Ideally, the extrapolated value $f_{\text {ext }}$, i.e., $\lim _{h \rightarrow 0} f(h)=f_{\text {ext }}$, should be equal to $f_{\text {the }}$, and $p$ should be equal to $p_{\text {theo. }} p$ is estimated through an iterative procedure; naming the three consecutively most refined discretizations as $h_{f}, h_{m}$, and $h_{c}$, such that $h_{f}<h_{m}<h_{c}$, $r_{m f}=\frac{h_{m}}{h_{f}}$ and $r_{c m}=\frac{h_{c}}{h_{m}}$, the observed $p$ can be calculated through a fixed point iterative scheme for equations (3.16):

$$
\begin{gathered}
p=\frac{1}{\ln \left(r_{m f}\right)}\left[\ln \left|\frac{f\left(h_{c}\right)-f\left(h_{m}\right)}{f\left(h_{m}\right)-f\left(h_{f}\right)}\right|+q(p)\right] \\
q(p)=\ln \left(\frac{r_{m f}^{p}-s}{r_{c m}^{p}-s}\right) \\
s=1 \times \operatorname{sign}\left[\frac{f\left(h_{c}\right)-f\left(h_{m}\right)}{f\left(h_{m}\right)-f\left(h_{f}\right)}\right]
\end{gathered}
$$

in which $q(p)=0$ as a first guess. If the solutions are in fact in the asymptotic region, three grids are sufficient to adequately obtain $p$; however, a minimum of four grids is required to demonstrate that $p$ is constant, and this result depends on variables such as the size of the coarsest grid resolution. $f_{\text {ext }}$ is calculated according to equation (3.19):

$$
f_{\text {ext }}=\frac{r_{m f}^{p} f\left(h_{f}\right)-f\left(h_{m}\right)}{r_{m f}^{p}-1}
$$


The estimated extrapolated error is the difference between a numerical value and the extrapolated value, as in equation (3.9). Finally, the fine GCI is defined on equation (3.20):

$$
G C I=\frac{F_{S} \times\left|f\left(h_{f}\right)-f\left(h_{m}\right)\right|}{r_{m f}^{p}-1}
$$

The Factor of Safety $F_{s}$ is assigned 1.25 when using this procedure and suggests a result for the $C G I$ with the $95 \%$ confidence interval; this value seems to works appropriately in studies involving a structured grid refinement (but not necessarily structured grids). For unstructured grid refinements, a more conservative value should be adopted $\left(F_{s}=3.0\right)$. To convert the GCI to $u_{h}$, an assumption about the error distribution must be made. If this is assumed to be Gaussian about the fine grid solution, $u_{h}$, which is related to the GCI through equation (3.21):

$$
u_{h}=\frac{G C I}{k}
$$

has a value of 2 for the expansion factor $k$. However, for well behaved and highly resolved problems, the error distribution is roughly a shifted Gaussian, which gives a more conservative value for $k$ (about 1.1 to 1.15 ).

When the observed $p$ over four or more grids are far from constant or noisy, i.e. an oscillating or even not monotonic convergence, the Least Square Method (LSM) for its determination must be taken. For difficult problems, more than four grids may be necessary, with stable results obtained with about six grids and a refinement ratio of approximately 2 . The simplest LSM works well and therefore is recommended in the Standard. The assumed one-term expansion error of the numerical solution is given by equation (3.15), and the LS approach is based on minimizing an objective function $S\left(f_{\text {ext }}, C, p\right)$, such that:

$$
S\left(f_{\text {ext }}, C, p\right)=\sqrt{\sum_{i=1}^{N_{g}}\left[f\left(h_{i}\right)-\left(f_{\text {ext }}+C h_{i}^{p}\right)\right]^{2}}
$$

in which the number of grids $N_{g}$ must be higher than 3. Setting the partial derivatives of $\mathrm{S}$ individually to zero leads to the following equations: 


$$
\begin{gathered}
f_{\text {ext }}=\frac{1}{N_{g}}\left[\sum_{i=1}^{N_{g}} f\left(h_{i}\right)-C \sum_{i=1}^{N_{g}} h_{i}^{p}\right] \\
\alpha=\frac{N_{g}\left[\sum_{i=1}^{N_{g}} f\left(h_{i}\right) h_{i}^{p}\right]-\left[\sum_{i=1}^{N_{g}} f\left(h_{i}\right)\right]\left[\sum_{i=1}^{N_{g}} h_{i}^{p}\right]}{N_{g} \sum_{i=1}^{N_{g}} h_{i}^{2 p}-\left[\sum_{i=1}^{N_{g}} h_{i}^{p}\right]^{2}} \\
N_{g} \sum_{i=1}^{N_{g}} f\left(h_{i}\right) h_{i}^{p} \log h_{i}-f_{\text {ext }} \sum_{i=1}^{N_{g}} h_{i}^{p} \log h_{i}-\alpha \sum_{i=1}^{N_{g}} h_{i}^{2 p} \log h_{i}=0
\end{gathered}
$$

and a standard deviation $\sigma$ defined as usual:

$$
\sigma=\sqrt{\frac{\sum_{i=1}^{N_{g}}\left[f\left(h_{i}\right)-\left(f_{e x t}+C h_{i}^{p}\right)\right]^{2}}{N_{g}-3}}
$$

The above procedures are based on the assumptions that (i) the grids must be in the asymptotic range so the leading term of the power series expansion is sufficient to estimate the error and (ii) the discretizations are geometrically similar. As previously stated, this is generally not the case for practical problems, in which complex mathematical models and lack of grid geometrical similarity occur; the errors for these anomalous cases, particularly when the observed order of convergence is not possible to obtain or not reliable, can be approximated according to one of the following expressions (ECA; HOEKSTRA, 2014):

$$
\begin{gathered}
f(h)=f_{\text {ext }}+C h \\
f(h)=f_{\text {ext }}+C h^{2} \\
f(h)=f_{\text {ext }}+C_{1} h+C_{2} h^{2}
\end{gathered}
$$

with the first two suitable for monotonically converging solutions, while the last can be used for non-monotonic convergence, such as the $C_{n}$ on figures 27 and 28. By using any of the equations (3.27), the procedure adopted is similar to the least square approach described earlier, including a minimum of four grids; however, a judgment must be made of the quality of the data fit. So, a data range $\Delta f$ is defined:

$$
\Delta f=\frac{\max f\left(h_{i}\right)-\min f\left(h_{i}\right)}{N_{g}-1}
$$

The error estimation is considered reliable if the solution is monotonically convergent with $0.5<p<2.1$ and if $\sigma<\Delta f$, in which case $F_{s}=1.25$. Otherwise, $F_{s}=3$. Ultimately, different expressions are adopted for the estimated uncertainty, based on $\sigma$ and $\Delta f$. For $\sigma<\Delta f$

$$
u_{h}=F_{s}\left[f(h)-f_{\text {ext }}\right]+\sigma+\left|f(h)-f_{\text {ext }}\right|
$$


while for $\sigma>\Delta f$ :

$$
u_{h}=3 \frac{\sigma}{\Delta f}\left\{\left[f(h)-f_{\text {ext }}\right]+\sigma+\left|f(h)-f_{\text {ext }}\right|\right\}
$$

The last equations guarantee that the uncertainties obtained for such anomalous cases have a $95 \%$ confidence level, as well (ECA; HOEKSTRA, 2014). A final note is that regardless of the expression - equation (3.21), (3.29), or (3.30) - the procedure can be used to estimate the uncertainty $u_{h}$ of any grid level, as long as the in the calculations follow the proper assumptions/satisfy the required hypotheses, so the uncertainties of any desired mesh can be systematically obtained.

\subsection{Observations about $u_{i}$ and $u_{d}$}

Numerical simulations generally contain inputs that come from experimental parameters that have associated uncertainties. To estimate the associated input uncertainty, two different methods are feasible, and the best choice depends on how the estimation process is approached: the local approach is concerned with the response of the system in a small neighborhood of a determined parameter, whereas the global considers this response in a large neighborhood.

The local approach for uncertainty propagation considers that all the input parameters are uncorrelated, such that the input uncertainty for a given result $S$ can be written as:

$$
u_{i}^{2}=\sum_{i=1}^{n}\left(\frac{\partial S}{\partial X_{i}} u_{X i}\right)^{2}
$$

in which $X_{i}$ is a given input parameter such that $\vec{V}=\left\{X_{1}, X_{2}, \cdots, X_{i}, \cdots, X_{n}\right\}$, and its associated uncertainty is $u_{X i}$. The partial derivatives, termed sensitivity coefficients, can be obtained through many techniques, such as analytic differentiation of the solution, or a numerical Finite Difference (FD) scheme. When the variable of interest has a simple (generally explicit) dependence on $X_{i}$, analytic differentiation is probably the most adequate approach; however, a more likely case is that the model is a complex simulation for which FD is the most pragmatical way. Approximating the sensitivity coefficient numerically through a second-order accuracy FD:

$$
\frac{\partial S}{\partial X_{i}}=\frac{S\left(X_{1}, \cdots, X_{i}+\Delta X_{i}, \cdots, X_{n}\right)-S\left(X_{1}, \cdots, X_{i}-\Delta X_{i}, \cdots, X_{n}\right)}{2 \Delta X_{i}}+O\left(\Delta X_{i}^{2}\right)
$$

Thus, to estimate the sensitivity of all $n$ input parameters, $2 n+1$ simulations are needed. In addition to issues related to incomplete nonlinear iteration, a difficulty of 
such numerical approximation is the choice of an appropriate perturbation size $\Delta X_{i}$ : for a too-large $\Delta X_{i}$, truncation errors are too large. On the other hand, for a $\Delta X_{i}$ too small, machine round off becomes significant due to subtractive cancellation in the numerator of equation (3.32).

The global approach for uncertainty propagation is appropriate to capture highly nonlinear behavior in the parameter space, a capability not feasible by the local, sensitivity method; perhaps the full Monte Carlo (MC) is the most reliable method for uncertainty analysis, but the number of numerical evaluations to perform a proper MC approach may lie from tents to thousands, making it extremely costly. An alternative is the Latin Hypercube Sampling (LHS) method, in which the cumulative probability distribution is divided into $n_{L H S}$ bands of equal probability, and each band has $n_{p}$ simulations, corresponding to the $n_{p}$ important parameters of the problem. Therefore, a total of $n_{L H S} \times n_{p}$ simulations is performed $\left(n_{L H S} \geq n_{p}+1\right)$, and for each of them, the parameters are randomly set but following certain rules. After the simulations, the $u_{i}$ can be estimated as follows:

$$
\begin{gathered}
\bar{S}=\frac{1}{n_{L H S}} \sum_{i=1}^{n_{L H S}} S_{i} \\
u_{i}^{2}=\frac{1}{n_{L H S}-1} \sum_{i=1}^{n_{L H S}}\left(S_{i}-\bar{S}\right)
\end{gathered}
$$

The LHS method needs an assumption about the distribution functions of the input variables; thus, as $u_{i}$ depends on such assumption, its sensitivity to it can be explored, and a certain amount of subjectivity is reasonably expected. Additionally, as care must be taken for the local approach, it must so for the global counterpart: a difficulty is regarding the adequate $n_{L H S}$ for appropriate determination of $u_{i}$

The input parameter uncertainty is assumed independent of the numerical uncertainty, a good assumption for small perturbations and FD sensitivity analysis. For global methods, $u_{i}$ might have a dependence on $u_{n}$ when coarse grids are used. Therefore, it is interesting to computed $u_{i}$ on the finest grid used to estimate $u_{n}$ if both uncertainties are comparable in size. When $u_{n} \ll u_{i}$, a coarser grid can be used.

The process used in the determination of experimental uncertainty is to calculate the uncertainties of the correlated measured variables and then combine them to form the uncertainty of a desired dependent variable. For a measured variable, the total uncertainty is influenced by many sources, which are grouped by several ways; one of them is to classify the uncertainties as either random - whose error source contribute to the variability of the measurement, or systematic - whose error sources remain fixed during the measurement.

The random uncertainty $u_{r j}$ of an $X_{j}$ variable is an estimate of the range of the random error for a measured variable, which is given by the standard deviation of the 
associated distribution of measurements; these measurements must be taken over the time frame and conditions that cover the variations in the variable to reflect its entire range of possible values. The systematic uncertainty $u_{s j}$ of a the same variable is obtained through the square root of the sum of all the squares of the systematic uncertainties for all the independent error sources $u_{k v}$ of that variable - equation (3.34) -, which are estimated from calibration data, analytic models, and previous experience, for instance:

$$
u_{s j}=\sqrt{u_{1 v}^{2}+u_{2 v}^{2}+\cdots+u_{k v}^{2}+\cdots}
$$

Those estimates are often made at some confidence level, which typically are representatives of the $95 \%$ limits of the possible values of the systematic error; hence a distribution must be assumed to obtain this percentage estimate, and the estimate is divided by the appropriate distribution factor, similar to the expansion factor $k$.

Such that an experimental result $D$ that is determined from $J$ measured variables as in equation (3.35):

$$
D=D\left(X_{1}, X_{2}, \cdots X_{j} \cdots X_{J}\right)
$$

has an associated experimental uncertainty $u_{d}$ given by equation (3.36):

$$
u_{D}=\sqrt{u_{r}^{2}+u_{s}^{2}}
$$

In which the random and systematic uncertainties, $u_{r}$ and $u_{s}$ of $D$ are given in terms of the input parameters as:

$$
\begin{aligned}
& u_{r}^{2}=\sum_{j=1}^{J}\left(\frac{\partial D}{\partial X_{j}} u_{r j}\right)^{2}+2 \sum_{j=1}^{J-1} \sum_{k=j+1}^{J} \frac{\partial D}{\partial X_{j}} \frac{\partial D}{\partial X_{k}} u_{r j k} \\
& u_{s}^{2}=\sum_{j=1}^{J}\left(\frac{\partial D}{\partial X_{j}} u_{s j}\right)^{2}+2 \sum_{j=1}^{J-1} \sum_{k=j+1}^{J} \frac{\partial D}{\partial X_{j}} \frac{\partial D}{\partial X_{k}} u_{s j k}
\end{aligned}
$$

The terms $u_{r j k}$ and $u_{s j k}$ are the covariance of the random and systematic uncertainties, respectively, and indicate the level of correlation between two variables; whereas usually the random uncertainties are considered to be independent (leading $u_{r j k}$ to zero), $u_{s j k}$ is determined by combining the elemental systematic uncertainties that arise from the same source. 


\subsection{Validation}

While code and solution verifications have no concern for the agreement of the simulation with experimental data, that is the concern of validation. Validation uncertainty $u_{v}$ is obtained through the knowledge of $u_{n}, u_{i}$, and $u_{d}$ : the determination of $u_{v}$ can be done through two approaches, which are associated with how $u_{i}$ was estimated (locally or globally). Therefore, once the uncertainties have been characterized, $u_{v}$ is obtained straightforwardly. Some observations, nonetheless, must be made.

When the variable of interest is a direct measure of an experiment, $D$ and $S$ generally have no shared variables, which leads to a straight evaluation of $u_{i}$ and $u_{d}$ as previously discussed. In this case, $u_{v}$ can be written according to equation (3.8). On the other hand, if the variable is determined from measurements, reference sources, and/or reduction equations, or if there are error sources shared by different variables (meaning nonzero covariances) equation (3.8) is substituted by (3.39).

$$
u_{v}=\sqrt{u_{n}^{2}+u_{i+d}^{2}}
$$

The determination of $u_{i+d}$ is particular to the case of interest. Despite having shared variables, the use of either local or global approaches implies that:

- the number of evaluations can be significant, so for a large number of input parameters, it is wise to identify the significant ones for the validation exercise and save some effort. Generally, the local approach requires fewer evaluations than the global; the later requires several evaluations sufficiently large such that the predictions of the average and standard deviation (uncertainty) can be resolved;

- the local approach assumes that $u_{i}$ can be evaluated from the nominal values of the input parameters (through the knowledge of the first order sensitivities), which may not be an appropriate assumption if the validation variable depends on them in a highly nonlinear fashion;

- the local approach does not require a full specification of the uncertainty distributions, as in the case of the global approach; the knowledge about the average value and the standard deviation is sufficient. However, without further assumption, the interval which $\epsilon_{m}$ falls to within a confidence percentage cannot be characterized. This can only be done by the global approach, as it requires specification about the distribution, and the higher the confidence level, the higher is the number of simulations required. If there is no sufficient knowledge to specify any of the distributions, a range of distributions can be utilized to evaluate the sensitivity of the validation to the distributional choices; 
- the global approach can be performed on a coarser grid if it is established the $u_{n}$ is significantly smaller than $u_{i}$, otherwise, $u_{v}$ will significantly increase (meaning that $u_{v}$ will be more related to computational limitations than to the uncertainties in the experiments).

The validation uncertainty is an estimate of the standard deviation of the combination of all errors except the modeling one. Thus, according to equation (3.7), while $E$ is an estimate of $\epsilon_{m}, u_{v}$ is the standard uncertainty of that estimate (i.e. $u_{v}$ can be viewed as $u_{\epsilon_{m}}$. Moreover, without the knowledge of its probability distribution, an interval within which $\epsilon_{m}$ falls cannot be estimated. Nonetheless, the following statements can be made. For instance, if:

$$
|E| \gg u_{v}
$$

then probably $\epsilon_{m} \approx E$, meaning that the model can be improved to reduce such error. On the other hand, if:

$$
|E| \leq u_{v}
$$

then probably $\epsilon_{m}$ is of the same order or less than $\left(\epsilon_{n}+\epsilon_{i}-\epsilon_{d}\right)$, meaning that the modeling error is within 'noise level' imposed by all the sources of uncertainty and 'dismissing' the need for model improvements, if the proposed model meets the designer needs.

Still, if a confidence level is required, the assumption about the probability distribution for $\epsilon_{m}$ allows the choice of a coverage factor $C_{f}$ such that the expanded uncertainty:

$$
U_{v \%}=C_{f} u_{v}
$$

can be defined so $E \pm U_{v X}$ defines an interval within which $\epsilon_{m}$ falls about $X \%$ confidence. For instance: (i) a uniform distribution with equal probability that $\epsilon_{m} \in \pm A$ leads to a $\sigma=\frac{A}{\sqrt{3}}$, so $100 \%$ of the population is covered for $C_{f}=1.73$; (ii) a triangular distribution symmetric about $\epsilon_{m}=0$ with base spanning from $-A$ to $A$ leads to a $\sigma=\frac{A}{\sqrt{6}}$, so $100 \%$ of the population is covered for $C_{f}=2.45$; and (iii) a Gaussian with standard deviation $\sigma$ has a $95.5 \%$ coverage for $C_{f}=2.00$, a $99.7 \%$ coverage for $C_{f}=3.00$, a $99.95 \%$ coverage for $C_{f}=3.50$, and a $99.99 \%$ coverage $C_{f}=4.00$. For the particular case of the global approach, a direct calculation of $C_{f}$ (or an equivalent, for a symmetric distribution) can be performed for sufficiently large number of samples. 


\section{Results}

The results are presented in terms of code and solution verification and later validation of both WLL and PLL; first, the wing formulation is thoroughly assessed and only after it the PLL is evaluated, as this was the natural course of development of this work.

To verify the proposed formulations, integral variables such as $C_{L}$ and $K_{T}$ were used as figures of merit. The cases for evaluation were chosen based on two criteria: (i) the availability of analytic solution and (ii) the capability of simulating different aspects of the numerical code developed, as recommended by the standard. The mesh refinement ratio used for uncertainty estimation started with 28 elements and ended with 904 with a refinement ratio of $\sqrt{2}$, and in addition to the level of discretization, clustering schemes were also evaluated; several authors report the used of spacially equispaced discretizations (KATZ; PLOTKIN, 2001; KERWIN; HADLER, 2010; EPPS; KIMBALL, 2013), while others claim that cosine clustering enhances the efficiency of discretization (PHILLIPS; SNYDER, 2000) as denser discretization is needed whenever discontinuities at the quarterchord exists (wings and blades tips and roots), for they give rise to rapid changes in $\Gamma$. Henceforth, both clustering schemes were evaluated: the equidistant and the cosine distributions.

The discretization uncertainties from the verification procedures are assumed to be the numerical uncertainties, as in most of the cases the absolute and relative errors between consecutive iterations were near machine zero (i.e., the iteration uncertainties were much lower than the discretization ones). However, the use of the most refined discretizations is time-consuming, even being prohibitive for the PLL case, as: (i) the matrix to be inverted has $(N \times Z)^{2}$ elements; (ii) although feasible, symmetry has not been implemented as the final interest is the application of the method to nonuniform advance velocity distributions, i.e., in behind hull wakes; and (iii) each HHSV must be discretized into a series of straight segments, which greatly contributes to the overall computation time. Therefore, the validation procedures were carried for an adequate level of discretization that comprises a balance between time and accuracy, and the corresponding numerical uncertainties were calculated as well (they are presented on the verification plots). They were added to other sources of uncertainty whenever available, but following the assumptions of uncertainty independence, i.e., no variables share the same error source. This reduces the validation uncertainty equation to (3.8); the validation plots, however, present the numerical and experimental errorbars separately. The input sources of error were treated by the local sensitivity analysis using the FD approach - equations (3.31) and (3.32) -, since it is a more straightforward and does not require information about the errors distributions and 
the establishment of the $n_{L H S}$ probability bands, as required by the LHS method. For all WLL simulations, at least two sets of data are presented: one considering simulations from either Star-CCM+ or XFOIL as the source of 2-D data and the other the results from the TFT; in terms of computation time versus accuracy, XFOIL is preferred over Star-CCM+, as it is much less time-consuming, so it would not add much to the overall LLT formulations. For the PLL, a third source was also considered whenever the blade section family was the NACA 66 TMB modified $\mathrm{a}=0.8$, as equations (2.36) could be used on $C_{n}$ and $C_{c}$; when the source of $2-\mathrm{D}$ was numerical (Star-CCM+ OR XFOIL), at least two input uncertainties existed, from $C_{n}$ and $C_{c}$ (whose associated uncertainties were assumed $u_{C_{n}}=1 \times 10^{-2}$ and $u_{C_{c}}=1 \times 10^{-4}$, given the uncertainty analysis performed previously, even though these figures do not thoroughly reflect the foils uncertainties during the entire flow regimes at which they are subject). Finally, depending on the reference, experimental and even other input uncertainties were provided for validation.

\subsection{Wing Lifting-Line}

For the WLL, four different cases were used as benchmark: (i) elliptically-loaded wings under potential flow, (ii) a NACA 0012 rectangular-planform under real flow, (iii) a flat-plate 45-deg sweptback wing under potential flow, and (iv) a flat-plate 10-deg dihedral wing under potential flow; although only case (i) has analytic solution, it is interesting to conduct at least an assessment of the convergence behavior and an evaluation of the correspondent discretization uncertainty for the other wings.

Some of the planforms used for verification also have been experimentally evaluated (given minor adaptations in their geometries and considering real flow conditions); while three of them were used, a fourth case, with variable-sweep angle distribution, named crescent wing, is also evaluated.

\subsubsection{Code and Solution Verification}

While figure 15 presented a discretization of an arbitrary wing for illustrative purposes, both clustering schemes are shown on figure 32 .

\subsubsection{Elliptically-Loaded Wings Under Potential Flow}

Classic lifting-line formulation establishes that under adequate assumptions - as small angles of attack and potential flows -, for an elliptic circulation distribution, Prandtl's equation of finite wing theory reduces to (CANTWELL, 2013):

$$
\Gamma_{0}\left[1-\left(\frac{2 y}{b}\right)^{2}\right]^{1 / 2}=\frac{1}{2} \frac{\partial C_{n}}{\partial \alpha}(y) c(y)\left[V_{\infty} \alpha(y)-\frac{\Gamma_{0}}{2 b}\right]
$$


Figure 32 - The two clustering evaluated: equidistant and cosine discretizations. Wing case.
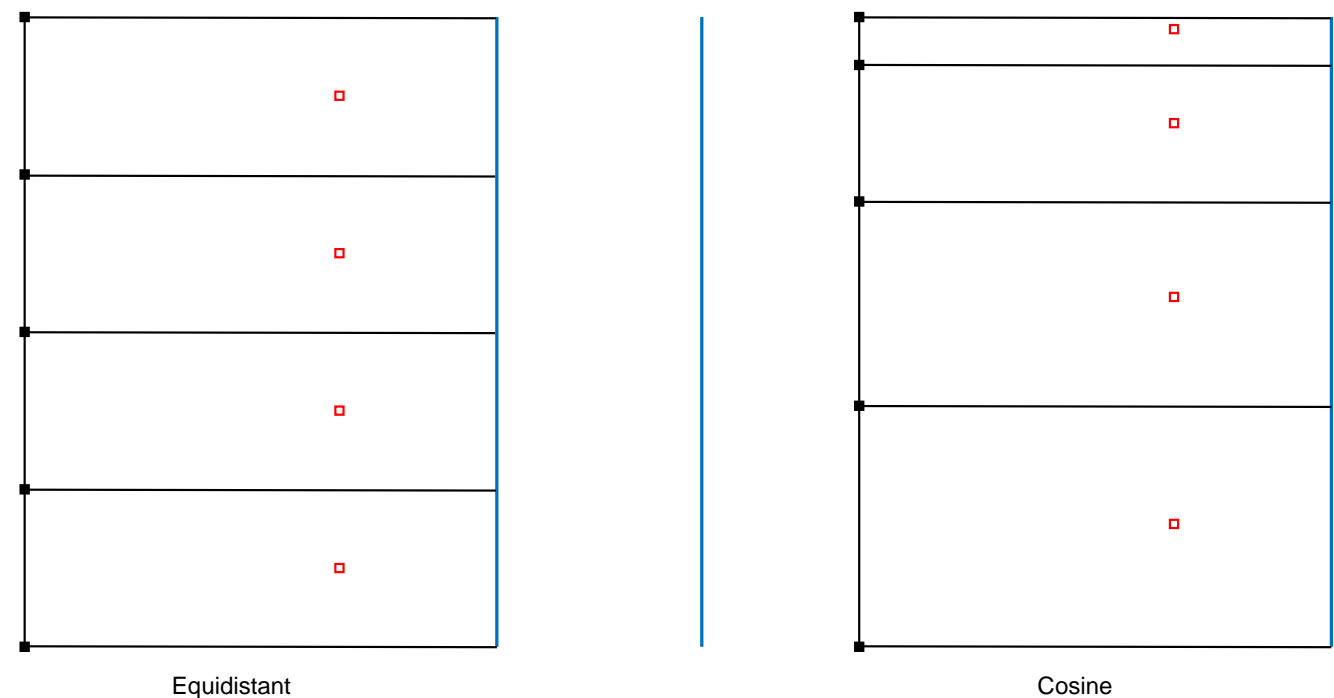

Source: Author

There are a variety of combinations of airfoils with different lift slopes, chord distributions and angle of attack distributions that can generate an elliptic lift distribution. For instance, if the wing cross-section and angle of attack are the same through the wing, the elliptic circulation can be obtained through a variable chord distribution. Solving equation (4.1) for such variable:

$$
\begin{gathered}
c(y)=c_{0}\left[1-\left(\frac{2 y}{b}\right)^{2}\right]^{1 / 2} \\
c_{0}=\frac{4 b \Gamma_{0}}{\left(2 b V_{\infty} \frac{\partial C_{n}}{\partial \alpha} \alpha_{0} \alpha_{0}-\frac{\partial C_{n}}{\partial \alpha} \Gamma_{0} \Gamma_{0}\right)}
\end{gathered}
$$

or, equivalently, for $\Gamma_{0}$ :

$$
\Gamma_{0}=\frac{2 b V_{\infty} \frac{\partial C_{n}}{\partial \alpha}{ }_{0} c_{0} \alpha_{0}}{\frac{\partial C_{n}}{\partial \alpha} c_{0}+4 b}
$$

Introducing the definition of lift coefficient and the KJT:

$$
C_{L}=\frac{L}{\frac{1}{2} \rho U_{\alpha}^{2} S}=\frac{\pi b}{2 V_{\infty} S} \Gamma_{0}=\pi \frac{b^{2}}{S}\left(\frac{1}{1+\frac{4 b}{\frac{\partial C_{n}}{\partial \alpha} c_{0}}}\right) \alpha_{0}
$$

Therefore, to achieve an elliptic lift distribution on an untwisted wing, the chord distribution should also be elliptic, whose planform area is $S=\frac{\pi}{4} \pi c_{0} b$. Assuming the lift 
slope result from the thin foil theory (i.e., $\frac{\partial C_{n}}{\partial \alpha} 0=2 \pi$ ), a flat-plate elliptic-planform wing with $b \approx 2.05 m$ and $c_{0} \approx 0.36$, under potential flow at an angle of attack of $4 \mathrm{deg}$, and cruise velocity $V_{\infty} \approx 59.31 \frac{\mathrm{m}}{\mathrm{s}}$ produces a theoretical lift coefficient $C_{L, t h e}$ of approximately 0.3447. Figure 33 presents the numerical behavior of $C_{L}$ for this wing as the grid is refined along with $C_{L, t h e}$ for reference. The plot on the right is a zoom in the most refined region, and it evidences the extrapolated values and the mesh uncertainties for the finest grids.

Figure $33-C_{L}$ versus normalized $h$ for code and solution verification. Elliptical planform wing, potential flow.
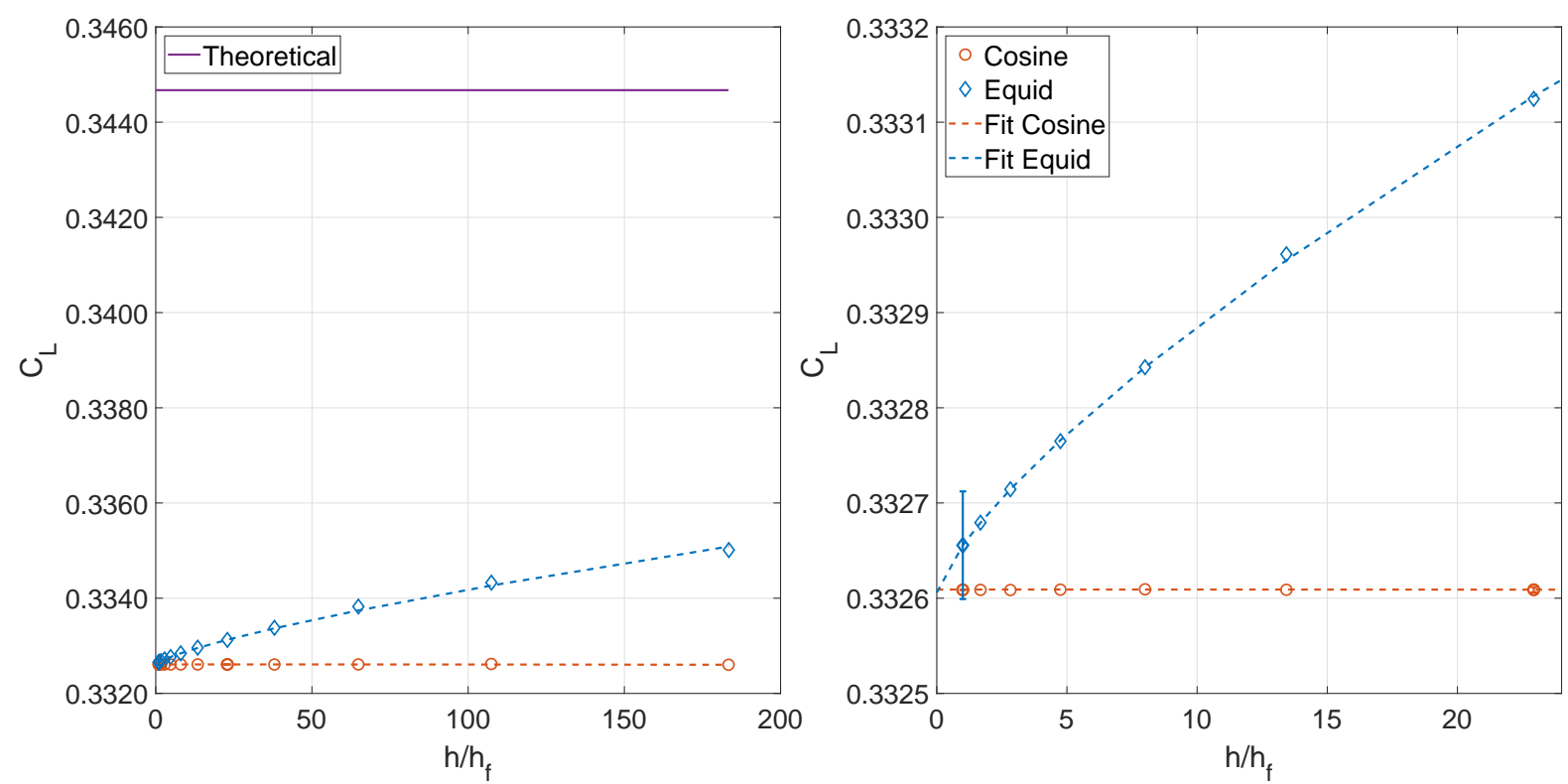

Source: Author

The extrapolated values and the rates of convergence are $C_{L, E \text {,ext }}=0.3326$ and $p_{E}=0.7501$ for the equidistant distribution and $C_{L, C, \text { ext }}=0.3326$ and $p_{C}=1.999$ for the cosine distribution. They produce respective associated uncertainties of $u_{h E}=5.6526 \times 10^{-5}$ and $u_{h C}=5.2748 \times 10^{-8}$. Apart from such differences, both discretizations produce extrapolated values whose relative differences from the exact value are approximately $3.50 \%$. While a rate of convergence near the theoretical rate $p_{\text {the }}$ was observed for the cosine clustering, the observed $p_{E}$ for the equidistant distribution is far from 2 . Therefore, for the cosine discretization the solution is truly on the asymptotic region for the finest grids, and the part of the code that was tested is correctly implemented; for the equidistant distribution, consequently, this order of convergence implies that grid convergence has not been carried to a sufficient refinement level.

Now, if the elliptic load is obtained by rather a variable lift slope distribution (i.e., wing sections with lift slopes different than $2 \pi$ but at the root), while both chord and angle of attack remain constant, from equation (4.1), such distribution can be written as: 


$$
\begin{aligned}
\frac{\partial C_{n}}{\partial \alpha}(y) & =\frac{\partial C_{n}}{\partial \alpha_{0}}\left[1-\left(\frac{2 y}{b}\right)^{2}\right]^{1 / 2} \\
\frac{\partial C_{n}}{\partial \alpha_{0}} & =\frac{4 b \Gamma_{0}}{\left(2 b V_{\infty} c_{0} \alpha_{0}-c_{0} \Gamma_{0}\right)}
\end{aligned}
$$

such that $\Gamma_{0}$ is given by equation (4.3) and, consequently, $C_{L}$ given by equation (4.4). Therefore, another way to achieve an elliptic lift distribution is to choose foils sections that follow the $\frac{\partial C_{n}}{\partial \alpha}$ distribution of equation (4.5) on an untwisted wing with rectangular planform. Assuming the same values for $b, c_{0}, \frac{\partial C_{n}}{\partial \alpha} 0, \alpha$, and $V_{\infty}$, and the same flow regime, the $C_{L, t h e}$ produced is approximately 0.2707 (due to the larger planform area). Figure 34 presents the numerical behavior of $C_{L}$ as the grid is refined along with $C_{L, t h e}$ for the reference. The plot on the right is a zoom in the most refined region, and it evidences the extrapolated values and the mesh uncertainties for the finest grids.

Figure $34-C_{L}$ versus normalized $h$ for code and solution verification. Elliptically-sloped wing, potential flow.
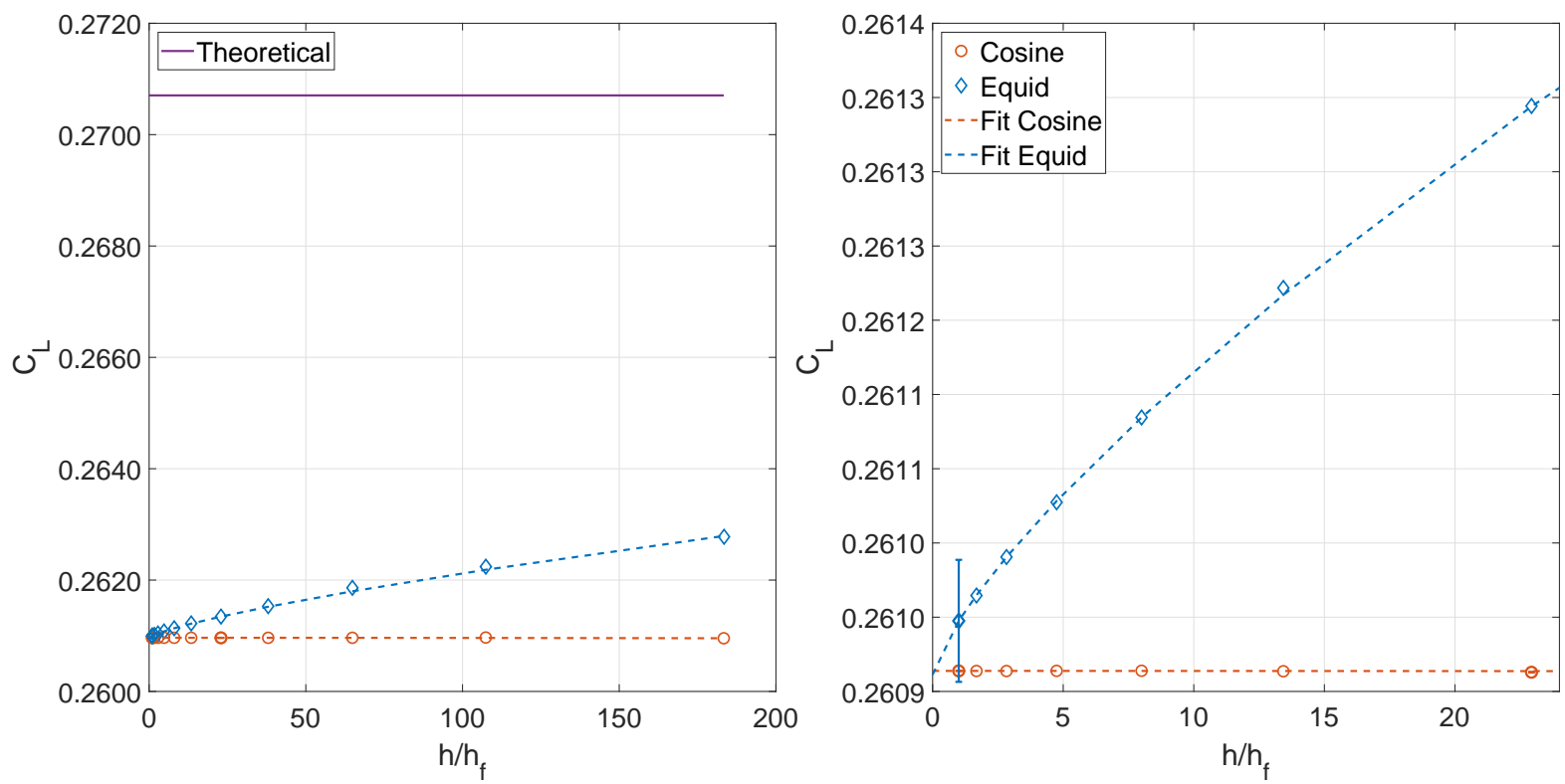

Source: Author

The extrapolated values and the rates of convergence are $C_{L, E, e x t}=0.2610$ and $p_{E}=0.7530$ for the equidistant distribution and $C_{L, C, e x t}=0.2630$ and $p_{C}=1.999$ for the cosine distribution. The respective uncertainties are $u_{h E}=4.1108 \times 10^{-5}$ and $u_{h C}=2.9053 \times 10^{-8}$. As in the previous case, both discretizations present a relatively low difference between the exact and extrapolated value (approximately $3.6 \%$ ), and $p_{C}$ is near the theoretical, while $p_{E}$ is far. Thus, as for lift slopes different than $2 \pi$ changes in the CP locations are necessary, this case tests the nonlinear iterative scheme; the results obtained for the cosine discretization indicate that the nonlinear schemes has been adequately implemented. 
Finally, a third way to produce elliptical loading is through a flat-plate rectangularplanform wing with 'elliptical' twist; setting constant chord and lift slope distributions on equation (4.1), the wing must be twisted in a fashion that the observed angle of attack as a function of the wingspan is given by:

$$
\begin{gathered}
\alpha(y)=\frac{4 b \alpha_{0}}{4 b+\frac{\partial C_{n}}{\partial \alpha} c_{0} c_{0}}\left[1-\left(\frac{2 y}{b}\right)^{2}\right]^{1 / 2}+\frac{\frac{\partial C_{n}}{\partial \alpha} c_{0} \alpha_{0}}{4 b+\frac{\partial C_{n}}{\partial \alpha} c_{0}} \\
\alpha_{0}=\frac{4 b+\frac{\partial C_{n}}{\partial \alpha} c_{0}}{2 \frac{\partial C_{n}}{\partial \alpha}{ }_{0} b V_{\infty} c_{0}} \Gamma_{0}
\end{gathered}
$$

Such that, again, $\Gamma_{0}$ is the same as in (4.3) and $C_{L}$ the same as in equation (4.4). Assuming the same values for $b, c_{0}, \frac{\partial C_{n}}{\partial \alpha}{ }_{0}, \alpha_{0}$, and $V_{\infty}$, and the same flow regime, the $C_{L, t h e}$ produced is the same as the previous case (0.2707). Figure 35 shows the convergence behavior of the numerical $C_{L}$ as the domain is refined along with $C_{L, t h e}$. The plot on the right is a zoom in the most refined region as previously, and it evidences the extrapolated values and the mesh uncertainties.

Figure $35-C_{L}$ versus normalized $h$ for code and solution verification. Elliptically-twisted wing, potential flow.
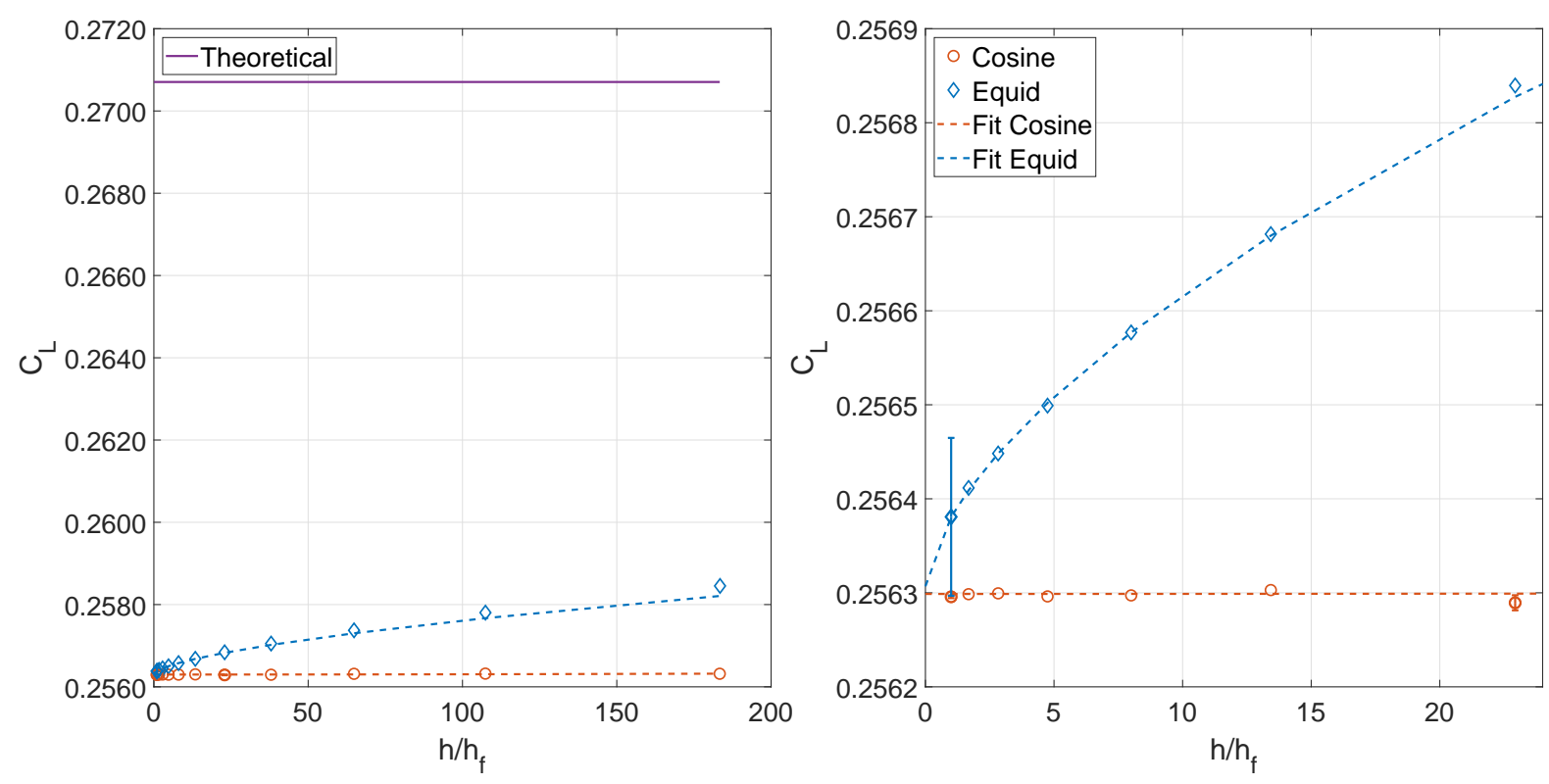

Source: Author

$C_{L, E, e x t}$ and $p_{E}$ are approximately 0.2563 and 0.6232 , whereas $C_{L, C, e x t}$ and $p_{C}$ are approximately 0.2563 and 2.0094; the uncertainties are $u_{h E}=8.3972 \times 10^{-5}$ and $u_{h C}=1.4083 \times 10^{-6}$. The same trend is observed as in the other two cases: the difference between the exact and extrapolated values are relatively low (approximately $3.6 \%$ ), and $p_{C}$ is close to 2 , while convergence $p_{E}$ is lower than 1 . Therefore, the code seems adequately verified (for the cosine discretization) for planforms that are not planar. 
Note that the presence of analytic values is important to the highlight that there will be a difference between the proposed model and Prandtl's regardless of the discretization level; this difference will likely create numerical discrepancies, and one the possible reasons is the different types of discretization for the two models: while in the former the horseshoe vortices are placed side by side and most of the wake is shed towards the free-stream direction, the latter superimposed vortices and the wake remains over the wing-planform plane despite the angle of attack. So, in addition to the natural limitation of the small angle-of-attack assumption, the differences observed on the charts of figures 33, 34, and 35 will likely increase with the increase in $\alpha$.

\subsubsection{Rectangular Wing Under Viscous Flow}

Solution verification could still be performed for other cases that assess important characteristics of the WLL formulation, even without the knowledge of the true value. A NACA 0012 rectangular-planform wing under viscous flow $\left(b=5.90 \mathrm{~m}, c_{0}=1.00 \mathrm{~m}\right.$, $V_{\infty} \approx 49.67 \frac{\mathrm{m}}{\mathrm{s}}$ ) is one of this cases, as information from a real, numerical, source of 2-D data must be used (which is different than the elliptically-sloped wing, as analytic and numerical/experimental sources of data are incorporated through different manners). The rectangular planform was chosen to minimize significant variations in $R e$ throughout the span, as otherwise large variations could lead to the propagation of errors that are intrinsic from the 2-D data. Figure 36 shows the convergence of $C_{L}$ as the grid is refined, with a zoomed-in region on the right.

Figure $36-C_{L}$ versus normalized $h$ for solution verification. Straight wing, viscous flow.
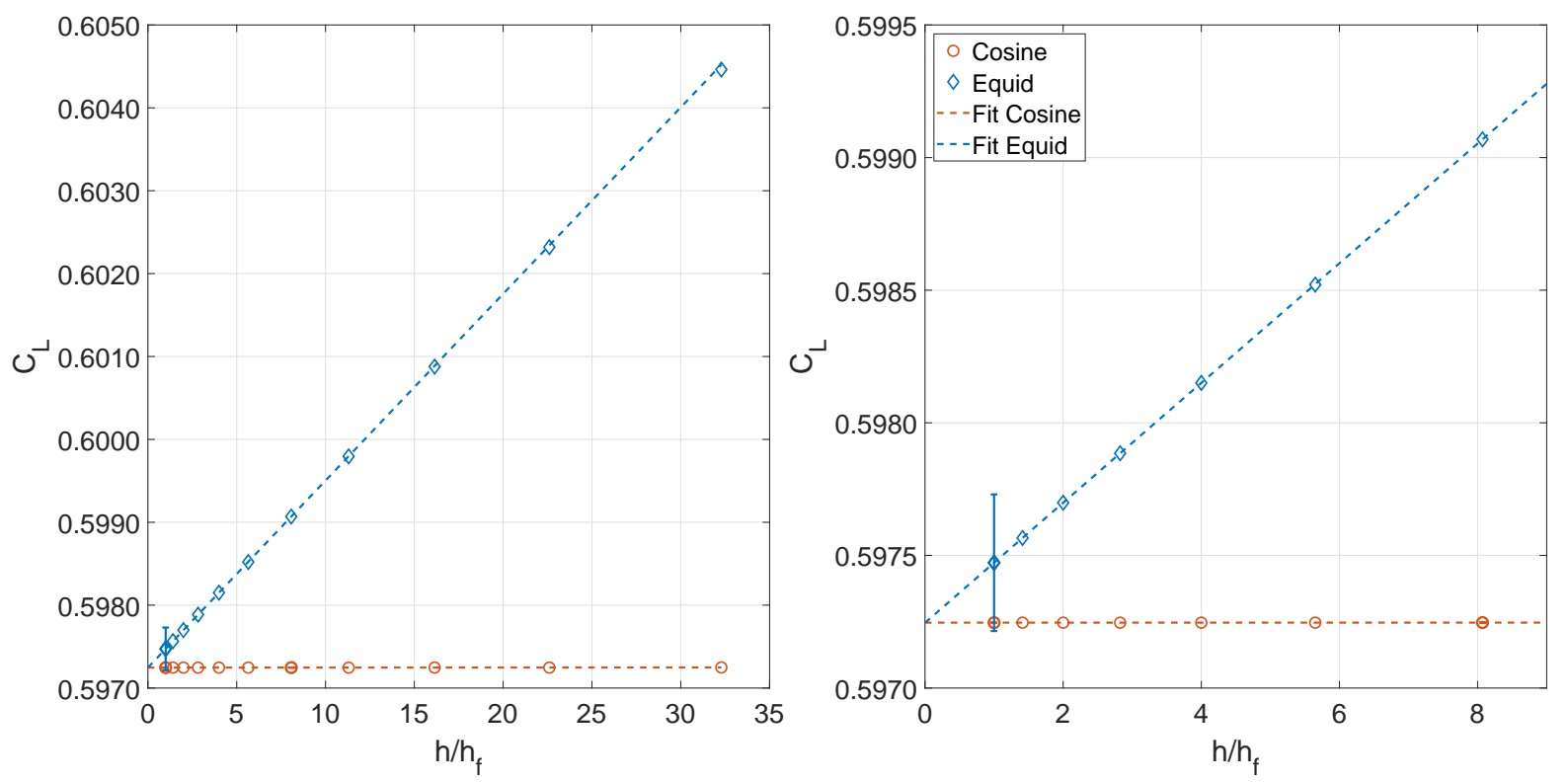

Source: Author

With the assumption that the modeling errors remain in the same order (thus the 
numerical solution is close to the true, unknown solution), it is observed that $C_{L, E, e x t} \approx$ $C_{L, C, \text { ext }} \approx 0.5972$, while $p_{E} \approx 0.9983$ and $p_{C} \approx 2.0000$, corroborating the advantage of using the cosine discretization over the equidistant one; $u_{h E}$ is significantly higher than $u_{h C}$ $\left(2.5756 \times 10^{-4}\right.$, and $9.8664 \times 10^{-9}$, respectively $)$. The inclusion of numerical/experimental 2-D aerodynamic data then does not contribute to an increase in the mesh uncertainties.

\subsubsection{45-deg Sweptback Wing Under Potential Flow}

Another case that is worth assessing is a flat-plate 45-deg sweptback wing under potential flow ( $b \approx 2.49 \mathrm{~m}, c_{0} \approx 0.51 \mathrm{~m}, V_{\infty} \approx 51.81 \frac{\mathrm{m}}{\mathrm{s}}$ ) as (i) the bound vortices have a variable chordwise location in space and (ii) the wing geometry itself makes three dimensional effects more prominent. Figure 37 shows the convergence of $C_{L}$ as the grid is refined.

Figure $37-C_{L}$ versus normalized $h$ for solution verification. 45-deg sweptback wing, potential flow.
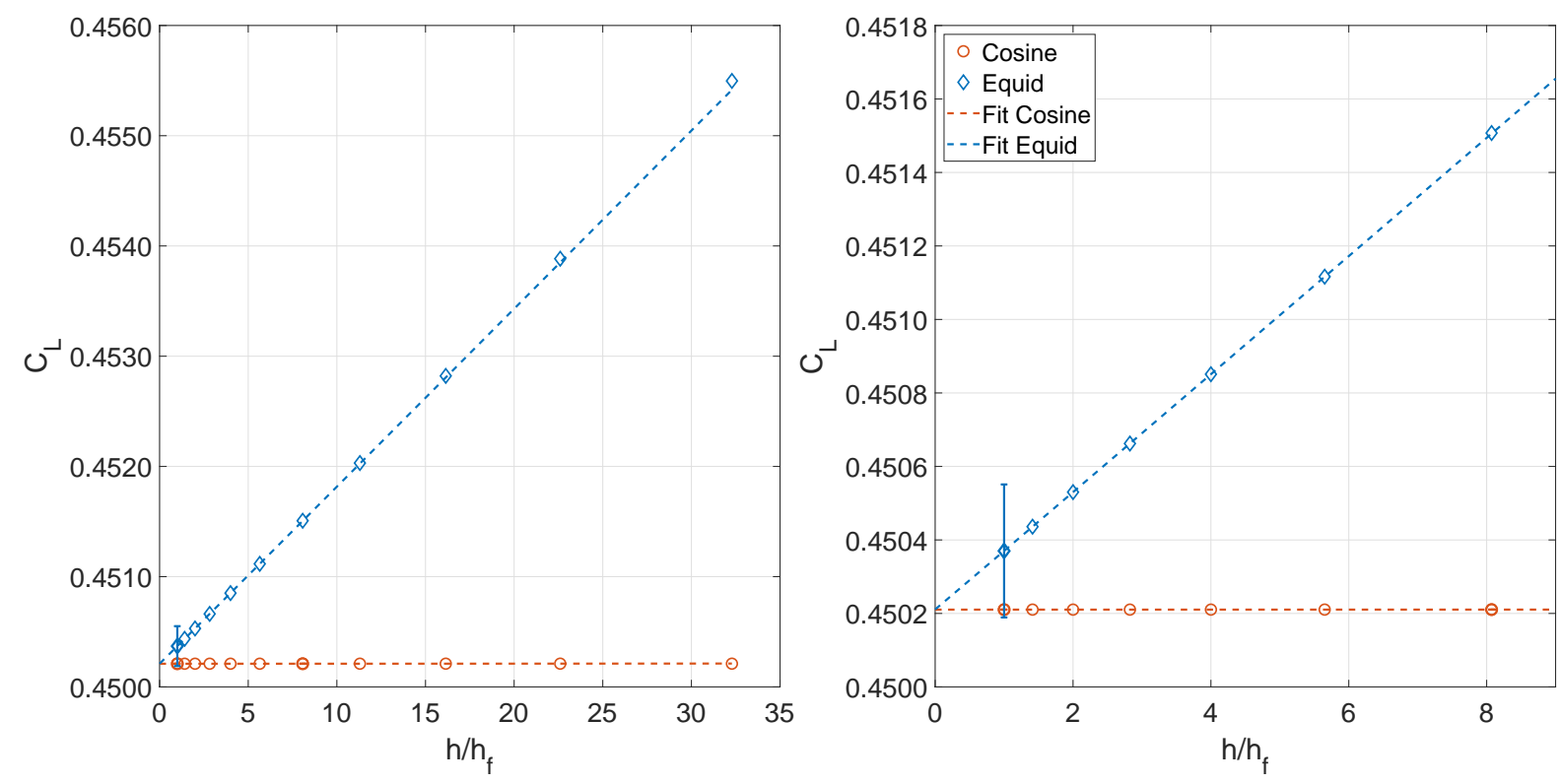

Source: Author

Again with the assumption of modelling errors of the same order of the ellipticallyloaded wings, the extrapolated values $C_{L, E, \text { ext }}$ and $C_{L, C, \text { ext }}$ are practically equal (0.4502), the convergence rate is better for the cosine discretization $\left(p_{E}\right.$ approx 1.0036 and $p_{C} \approx$ $1.9846)$, and it presented an observably lower uncertainty $\left(u_{h E}=1.8094 \times 10^{-4}\right.$ and $\left.u_{h C}=2.0100 \times 10^{-9}\right)$. According to the results for the cosine discreization, the formulation seems capable of including some degree of 3 -D effects through sweep angles. 


\subsubsection{10-deg Dihedral Wing Under Potential Flow}

A final verification case is for a flat-plate 10-deg dihedral wing under potential flow $\left(b=5.90 \mathrm{~m}, c_{0}=1.00 \mathrm{~m}, V_{\infty} \approx 21.15 \frac{\mathrm{m}}{\mathrm{s}}\right)$; such case assesses one last geometric variation of interest for the current wing formulation, when the wing sections are not on the same plane. Figure 38 shows the convergence of $C_{L}$ as the grid is refined.

Figure $38-C_{L}$ versus normalized $h$ for solution verification. 10-deg dihedral wing, potential flow.
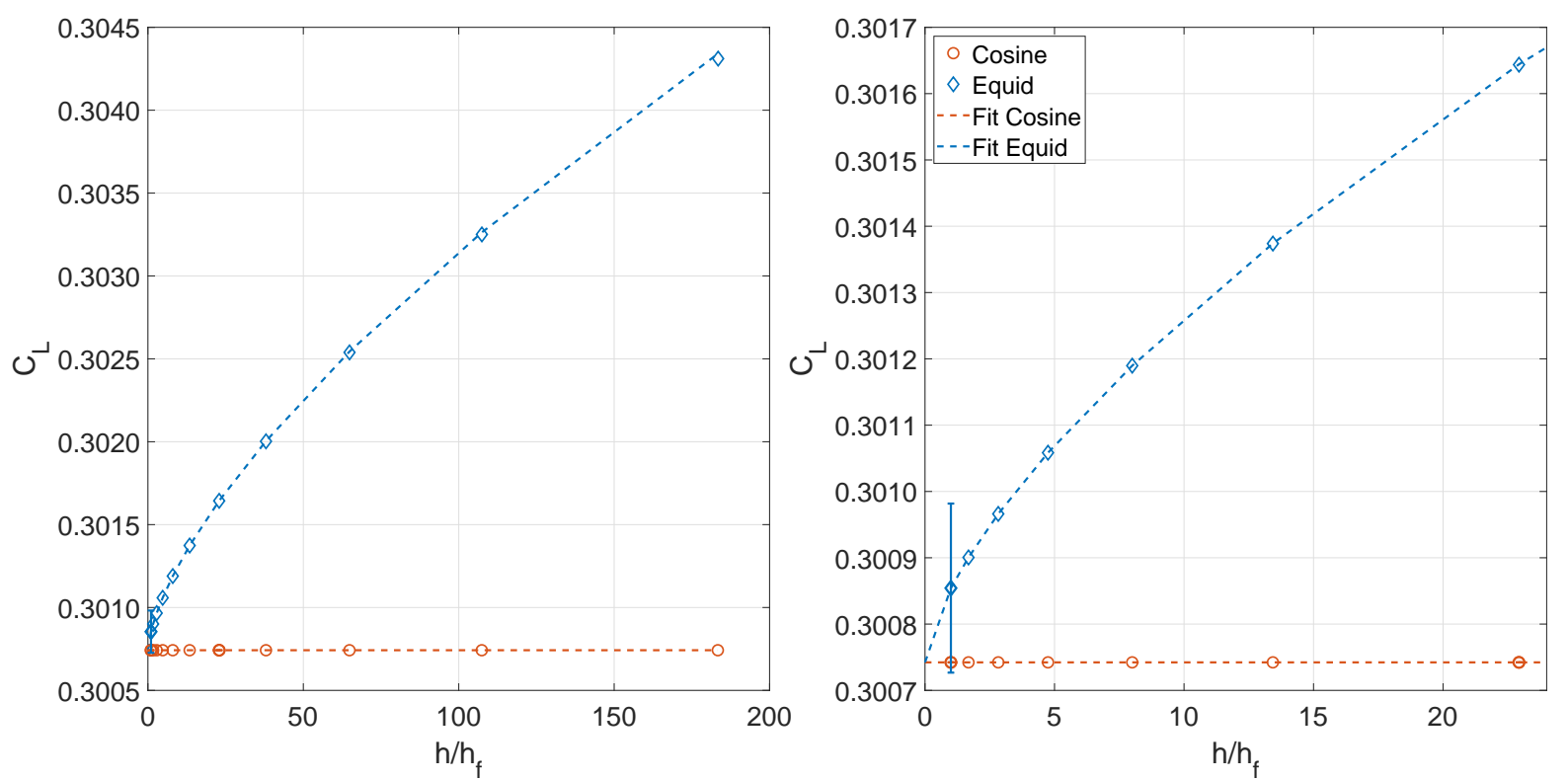

Source: Author

As observed on all other cases, the extrapolated values are for both discretizations are practically equal $\left(C_{L, E, \text { ext }} \approx C_{L, C, \text { ext }} \approx 0.3007\right)$, the orders of convergence follow the previously observed trends $\left(p_{E} \approx 0.6655\right.$, while $\left.p_{C} \approx 2.0000\right)$, and the mesh convergence uncertainties are significantly lower for the cosine clustering $\left(u_{h E}=1.2749 \times 10^{-4}\right.$ and $\left.u_{h C}=1.4808 \times 10^{-11}\right)$. The conclusion about the capability of including some degree of 3-D effects on the WLL also holds through dihedral angles.

\subsubsection{Conclusions about Code and Solution Verifications}

The code and solution verification procedures for the WLL lead to the following conclusions:

- errors are within a few percentages of the analytic values, which evidences that the code presents relatively small errors that are associated with modeling limitations;

- although there is no analytic solution for all cases assessed, given the same observed convergence trends, the same order of error is expected for them; 
- the inclusion of a source of 2-D data and (at least some degree) of sweep and dihedral angles do not seem to 'pollute' the numerical solution, meaning that the discretization uncertainties do not increase due them;

- clearly, the cosine clustering is the most appropriate, as the $p_{C} s$ obtained are always close to the theoretical one, while with the equidistant clustering $p_{E} s$ are lower by one order of magnitude. According to the standard, the equidistant discretization probably has not been carried to a sufficient level;

- the choice of the cosine discretization is corroborated by the order of magnitude of the uncertainties: while $O\left(u_{h C}\right) \approx 1 \times 10^{-6}$ in the worst case, $O\left(u_{h E}\right) \approx 1 \times 10^{-4}$ in the best case.

\subsubsection{Validation}

Once verified, the WLL is validated against several experimental data from the literature (WEBER; BREBNER, 1951; DAM; VIJGEN; HOLMES, 1991; APPLIN, 1995). Given the trends obtained from the verification processes, cosine clustering with $N=112$ was set for the numerical simulations; this combination of clustering and discretization level, as shown on the verification plots figures - 33, 34, 35, 36, 37, and 38, plots on the right - , have an associated numerical uncertainty $u_{n}$ in the order of $1 \times 10^{-5}$, which has been used on the uncertainty propagation of both $C_{L}$ and $C_{D}$ (although not presented, the mesh convergence trends of both coefficients are quite similar, if not better for $C_{D}$ ). Finally, for all the simulations in which the TFT was used, a constant value of viscous drag coefficient $C_{D_{0}}$ has been added to the overall wing drag.

\subsubsection{Elliptical Planform Wing}

The first validation case is for an almost-elliptical planform wing, similar to the one used for verification, but with a rectangular inboard section added; therefore, whereas both $b$ and $c_{0}$ remain unchanged, from 0 to approximately $0.183 \mathrm{~m}$ in the spanwise direction the planform is straight, and from approximately $0.183 \mathrm{~m}$ to $1.0272 \mathrm{~m}$ the planform is elliptical. Additionally, NACA 0012 airfoils compose the wing sections. A drawing of the wing planform is presented in figure 39.

The experiments (DAM; VIJGEN; HOLMES, 1991) have been conducted at $R e_{\infty} \approx$ $1.35 \times 10^{6}$ with respect to $c_{0}$, or equivalently $1.09 \times 10^{6}$ with respect to the mean aerodynamic chord. Boundary-layer transition mechanisms were placed along the wing span - in the free-stream direction - at a $5 \%$ chord length distance from the leading-edge over both upper and lower surfaces to ensure turbulence through almost the entire wing. The increment in subsequent angles of attack was $0.5 \mathrm{deg}$ for most of its range. At each AoA, balance readings were taken over a period of $20 s$ at a rate of 5 data sampling cycles/s, giving random 
Figure 39 - Planform of the elliptical wing.

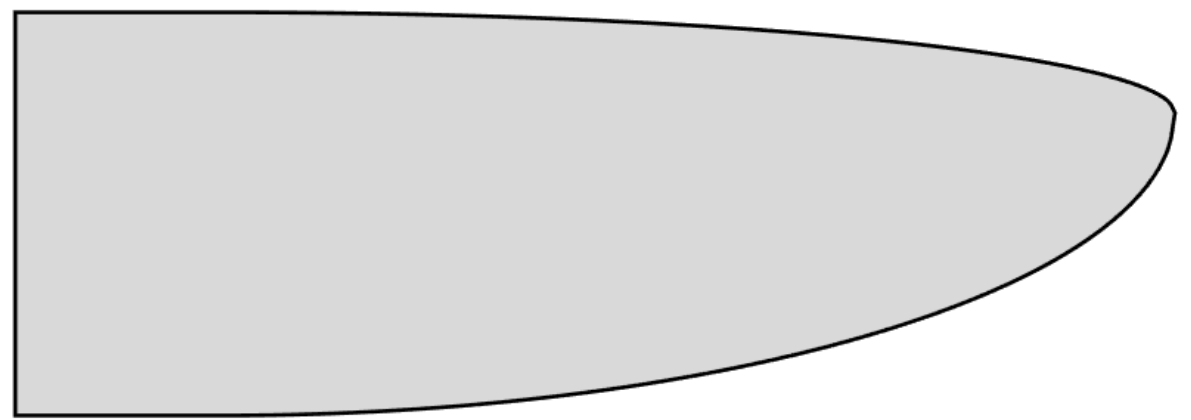

Source: Author

uncertainties of $u_{R, C_{L}} \pm 0.001$ and $u_{R, C_{D}} \pm 0.0003$. The uncertainties in the measurement of $C_{L}$ and $C_{D}$ are based on the accuracy of the internal balance, the AoA measurement, and the dynamic-pressure transducer. The balance maximum errors were $\pm 0.26 \%$ of full axial load and $\pm 0.13 \%$ of full normal load. The dynamic pressure had an accuracy of $\pm 0.04 \%$ of full scale, and the AoA was determined with an electronic inclinometer, providing an uncertainty of $\pm 0.025 \mathrm{deg}$. Finally, the maximum uncertainties in $C_{L}$ and $C_{D}$ for $\alpha=0$ deg are $u_{S, C_{L}}= \pm 0.001$ and $u_{S, C_{D}}= \pm 0.0004$, and $u_{S, C_{L}}= \pm 0.002$ and $u_{S, C_{D}}= \pm 0.0012$ for $\alpha=10 \mathrm{deg}$.

As the other uncertainties do not contribute substantially to $u_{i}$, only the uncertainty in the AoA has been used in the calculation of $u_{v}$, and in the case in which Star-CCM+ was the source of 2-D data, its uncertainties have been added to the overall one. However, only $C_{n}$ data was used due to the large variations in $R e$ across the wing; therefore a constant $C_{D_{0}}$ equals to 0.01293 (obtained from Star-CCM+) was added to the induced drag coefficient $C_{D_{i}}$, regardless of the angle of incidence. This same $C_{D_{0}}$ value was also added to the $C_{D_{i}}$ calculated through the WLL-TFT combination. The comparison between numerical and experimental data and between the numerical data themselves is presented in figure 40 .

$C_{L} \times \alpha$ shows that, in terms of numerical simulations, the differences between using the TFT or Star-CCM+ as the source of 2-D data become more evident as the AoA increases; the simulations in which Star-CCM+ is used are closer to the experimental data. However, the maximum difference is no higher than $10 \%$, which can bring to the discussion about whether a designer should consider the time taken to generate the foil database in detriment to the improvement in accuracy; such order of difference might even be close to the modeling error discussed during the verification process. In terms of $C_{D}$, however, the differences are higher more evident: while the agreement between the two numerical results worsens with the increase in $\alpha$, the simulations using Star-CCM+ agree way better to experimental results; in this case, the maximum difference between the numerical methods 
Figure $40-C_{L}$ and $C_{D}$ versus $\alpha$. Elliptical wing.
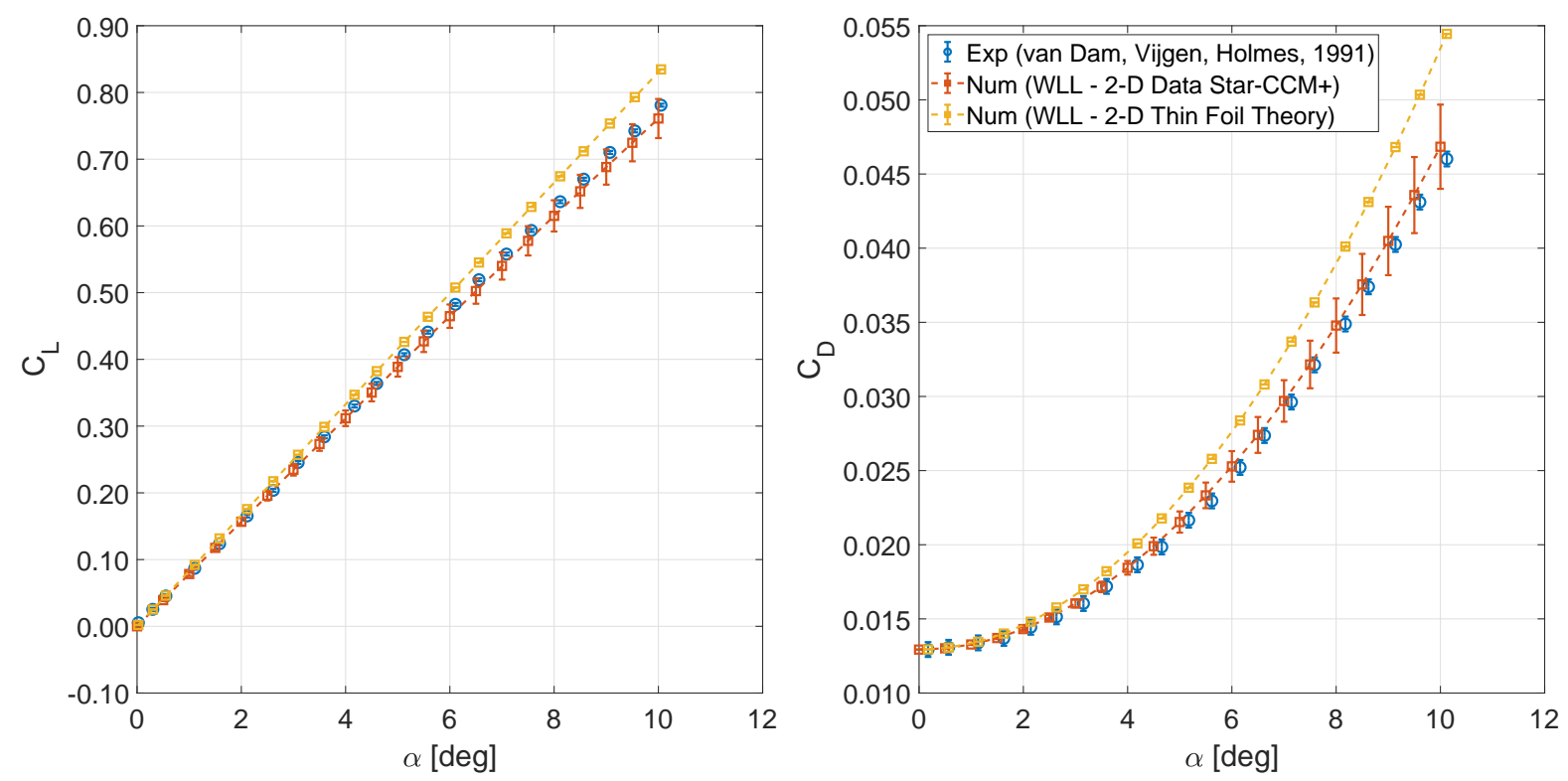

Source: Author

is about $16 \%$. Comparing the closest numerical and the experimental data, the increase in $\alpha$ also increases their dissidence, especially in terms of $C_{L}$ : the maximum relative difference for this coefficient is lower than $3.0 \%$, while for $C_{D}$ such difference is lower than $2.0 \%$. A possible explanation for the observed trends is the presence of three-dimensional effects on the flow, which become more prominent at higher $\alpha$. Still, the agreement is reasonably satisfying.

\subsubsection{Crescent Wing}

A second validation case is for a crescent wing, which has variable-sweep angle distribution and thus demonstrates the accuracy of the method in simulating swept wings under the influence of viscous flows. The difference between this wing and the elliptical is only the sweep angle distribution. The experimental data are also from the same source (DAM; VIJGEN; HOLMES, 1991), meaning that the experimental set-up and associated uncertainties remain the same; a minor difference happens in the placement of the trips to enforce turbulence. A drawing of the wing planform is presented in figure 41.

The sources of aerodynamic data were again Star-CCM+ and TFT; they were accounted in the same way as for the elliptical wing. For this wing, in terms of $C_{L}$ the same trend occurs between the two numerical simulations: $C_{L}$ is overestimated when the TFT is used in comparison to the simulations having tar-CCM+ as the source of 2-D data; the differences increase with the AoA and the maximum difference between the two is about $8 \%$. This time, however, there is not as substantial improvement from the Star-CCM+ source over the TFT source in comparison to the experimental data; in fact, 
Figure 41 - Planform of the crescent wing.

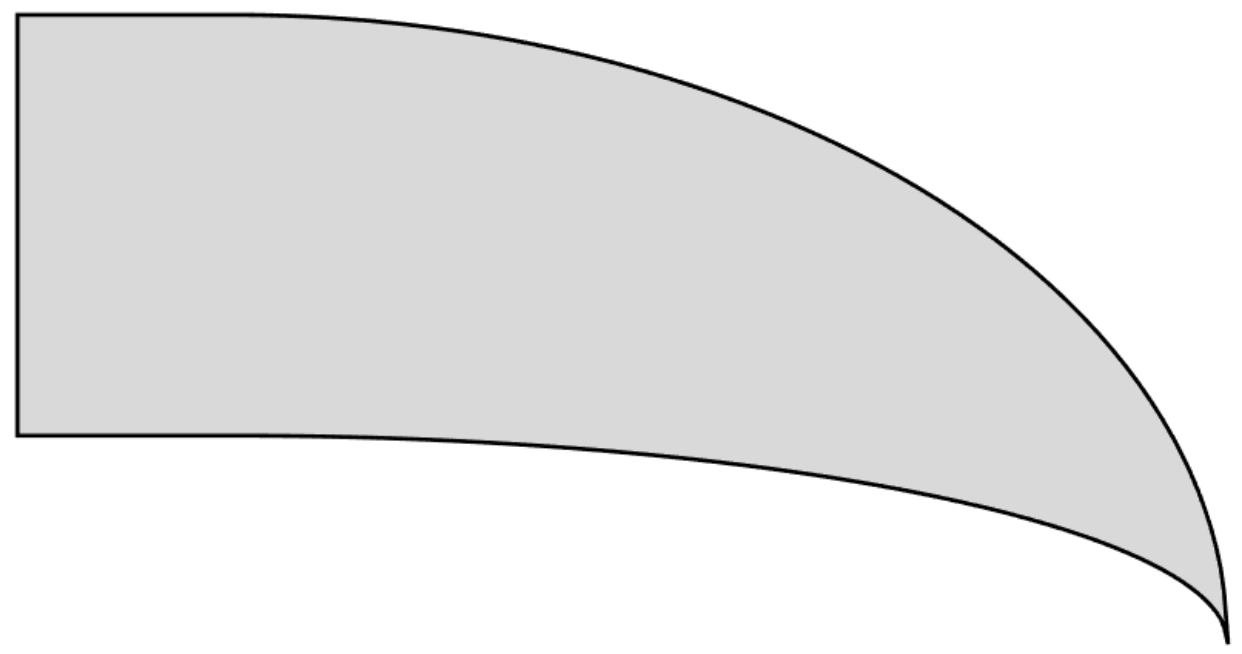

Source: Author

the results obtained with the use of the TFT are closer to the experimental data (the maximum relative differences between numerical and experimental data are about 3.0\% for the TFT data and $4.6 \%$ for the Star-CCM+ data). Besides, in terms of $C_{D} \times \alpha$, both numerical methods present slightly different results. So the advantages of using numerical 2-D data for this case are uncertain.

Even though the differences between the closest PLL and the experimental data seem subtly worse than in the elliptical wing, the agreement still seems satisfactory (a maximum difference of less than $5.0 \%$ in $C_{L}$ and less than $6.0 \%$ in $C_{D}$ ). This is a suitable result since the planform geometry incurs 3 -D effects that are more present, and possibly cannot be adequately captured by LLT methods.

\subsubsection{Rectangular Wing}

A third validation case is for the rectangular wing used in the verification procedure - figure 43. The experiments (APPLIN, 1995) have been conducted in the Langley Subsonic Tunnel at a range of Reynolds numbers with turbulence enforced at about $5 \mathrm{~cm}$ downstream the wing leading edge as to have essentially turbulent flows. The model was mounted vertically on a six-component strain-gauge balance that could be rotated throughout the AoA range, whose measure was detected by a digital shaft encoder and provided accuracy to within $\pm 0.02 \mathrm{deg}$. The data acquisition system averaged 20 of the samples into each data point, although no uncertainty about $C_{L}$ and $C_{D}$ was presented. Finally, for the current validation, $R e_{\infty} \approx 2.11 \times 10^{6}$ was used.

For the WLL, the uncertainty in $\alpha$ has been propagated in addition to the mesh uncertainty. For the simulations which used Star-CCM+, the correspondent uncertainties have also been added, but at this time, no 'frozen' $C_{D_{0}}$ has been added to the $C_{D_{i}}$ as the 
Figure $42-C_{L}$ and $C_{D}$ versus $\alpha$. Crescent wing.
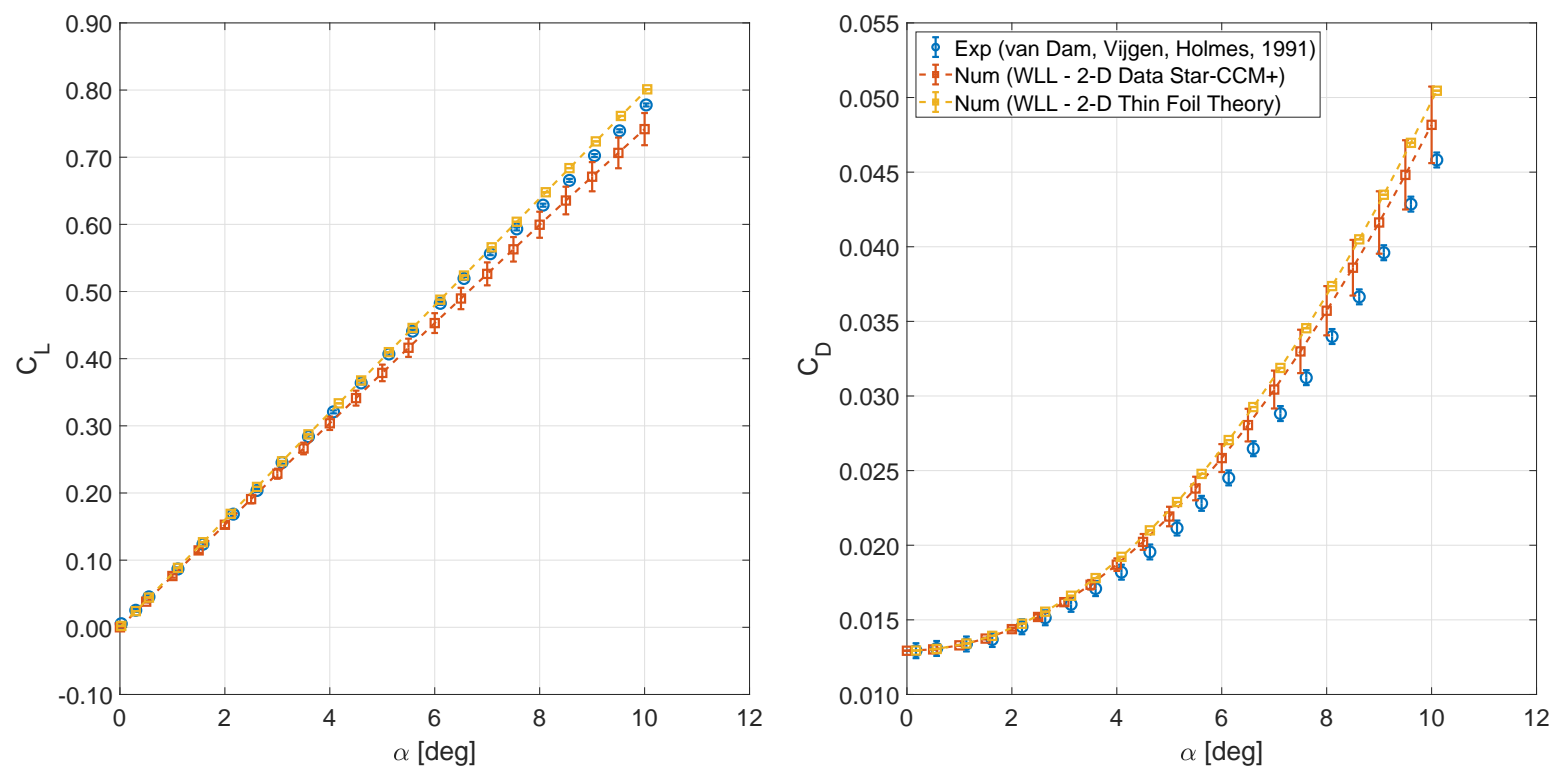

Source: Author

Figure 43 - Planform of the rectangular wing.

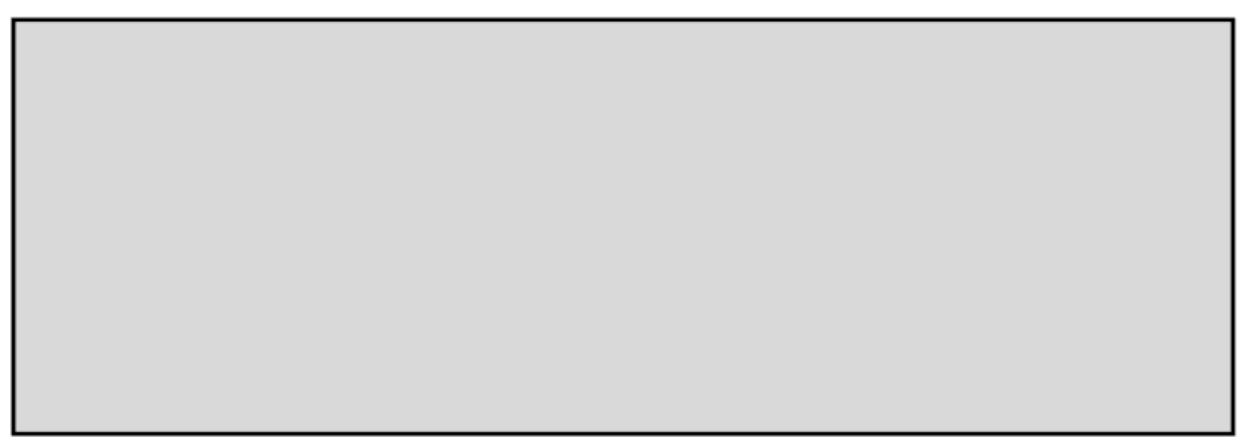

Source: Author

variations in Reynolds throughout the wing are minor: $C_{n}$ and $C_{c}$ have been used instead. Figure 44 shows the trends of $C_{L} \times \alpha$ and $C_{D} \times \alpha$ for this wing.

In terms of $C_{L}$, for most of the $\alpha$ range, there is a strong agreement between the numerical methods. Such agreement is lost for high $\alpha$, as near these regions the viscous effects start taking place, so the $C_{n}$ values start diverging from the TFT; the highest difference happens for $\alpha=15 \mathrm{deg}$, and again the results are better when Star-CCM+ is used. In terms of $C_{D}$, the relative differences are generally higher (with a maximum of approximately 11\%) and higher values for the simulations that used Star-CCM+ have been obtained; this is an expected trend, since lift is practically equal for both cases, meaning that these differences are associated with the inclusion of $C_{c}$. Note, however, that either the local coefficients or the modeling errors are possibly higher than they should, as the 
Figure $44-C_{L}$ and $C_{D}$ versus $\alpha$. Rectangular wing.
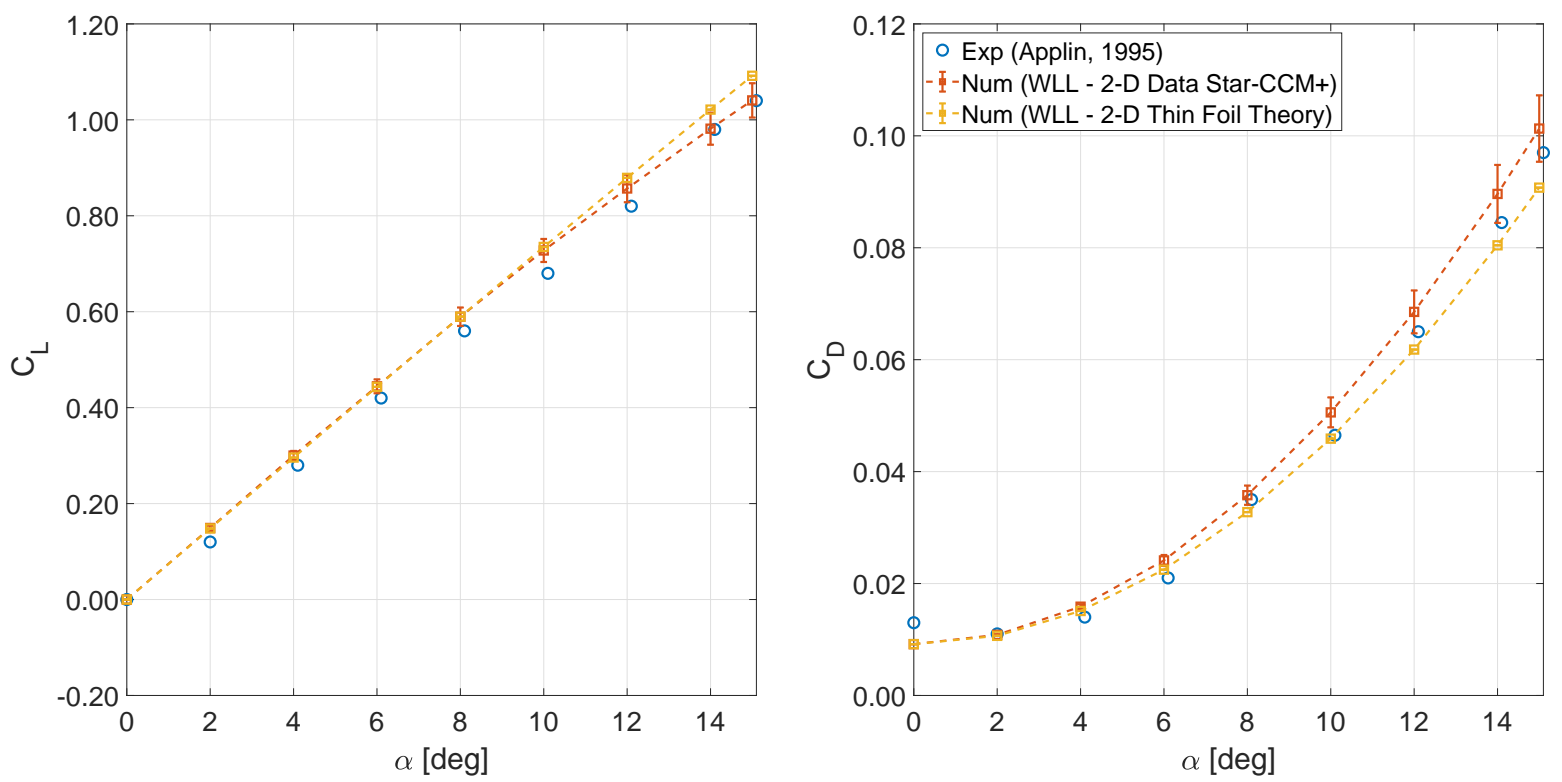

Source: Author

$C_{D}$ for the Star-CCM+ based simulation overestimates the experimental values, while for the TFT case, those values are underestimated.

One important feature is about the satisfactory $C_{L}$ agreement for relatively high values of the AoA: some discrepancy is observed near $\alpha=10 \mathrm{deg}$, but for the Star-CCM+ base simulation, the increase in the AoA in facts brought a better agreement. For this set of simulations, the maximum $C_{L}$ relative difference between numerical and experimental values is about $6.6 \%$. For $C_{D}$, this difference is about $8.0 \%$

\subsubsection{45-deg Sweptback Wing}

The last validation case is for the 45-deg sweptback wing also presented during the verification procedure (figure 45 ), with the difference that the RAE 101, $12 \% \frac{t_{0}}{c}$ airfoil has been used in place on the flat plate. For the experiments (WEBER; BREBNER, 1951), pressure measurements have been made along the wingspan through flush holes on both upper and lower surfaces. The range of AoA was from 0 deg to $10 \mathrm{deg}$, and $R e_{\infty} \approx 1.68 \times 10^{6}$. Ultimately, the chordwise pressure distributions have been graphically integrated so as the normal and tangential forces have been obtained; from them, the lift and drag coefficients have been calculated. No uncertainties have been provided for the experimental data or set-up.

For the lifting-line, the source of 2-D data was the software XFOIL (DRELA; YOUNGREN, 1989) (viscous formulation), with its associated assumed uncertainties in $C_{n}$ and $C_{c}$. The trends for $C_{L}$ repeat as in the other cases: there is an increasing discrepancy 
Figure 45 - Planform of the 45-deg sweptback wing.

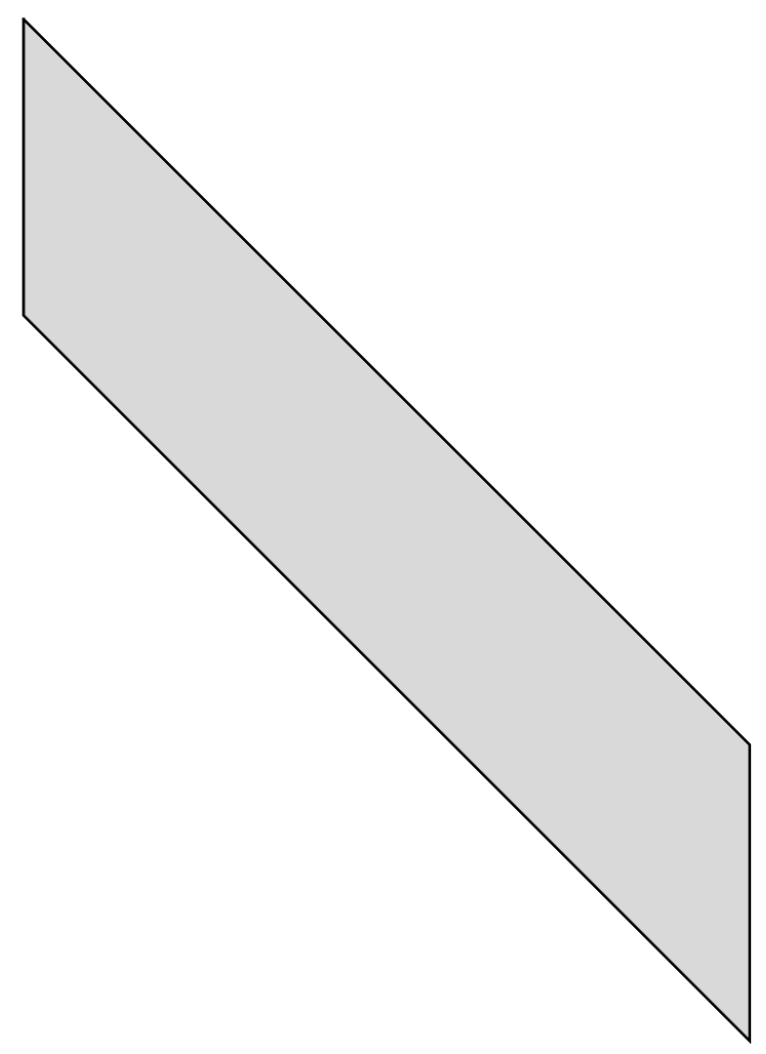

Source: Author

between the two numerical solutions with the AoA (maximum relative difference of $2.0 \%$ ) and the agreement with experimental data is better for the simulations that used the Star-CCM $+2-\mathrm{D}$ data. For $C_{D}$, however, while the agreement is better when viscous effects are not considered on low $\alpha$, this is not the case for high values. Comparison with experimental data reveals that the maximum $C_{L}$ difference is about $3.0 \%$ while the maximum $C_{D}$ difference is almost $90 \%$ (near $\alpha=2 \mathrm{deg}$ ), meaning that the simulations provide reasonably close results for $C_{L}$, but not for $C_{D}$. A possible explanation for such discrepancy is the source of 2-D data as its importance has been discussed in academic works (CHREIM et al., 2017) and throughout the text: for high $\alpha$, there is a predominance of induced drag - explaining the agreement -, but as $\alpha$ decreases, the contribution of the viscous drag is more substantial, and differences might arise if the 2-D data are not sufficiently accurate.

\subsubsection{Validation Tables and Conclusions about Validation}

A formal validation, in form of tables containing numerical (Star-CCM+ or XFOIL as source of 2-D data) and experimental values along with the respective uncertainties (whenever available) is presented for the wing cases discussed. Three values of the angles of attack where used without any regards to the distinction among types of flow regimes, 
Figure $46-C_{L}$ and $C_{D}$ versus $\alpha$. 45-deg sweptback wing.
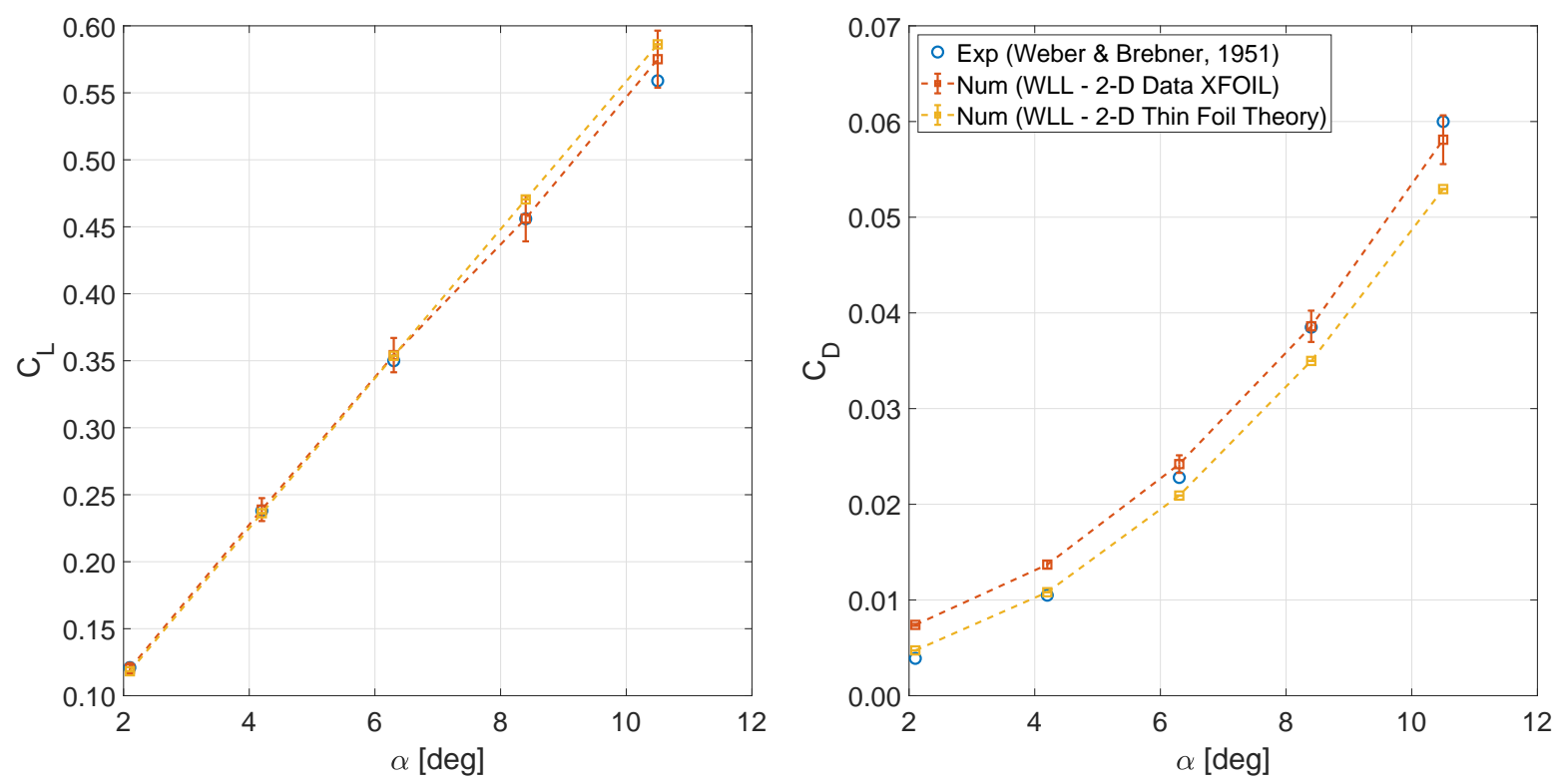

Source: Author

although the large angles of attack $\alpha_{L}$ were always the largest possible, aiming at $\alpha \geq 10$ deg region - off the small disturbance approximation (HALL, 2018) - whenever possible, while the small $\alpha_{S}$ and medium $\alpha_{M}$ were in the $\alpha<5 \mathrm{deg}$ and $5 \mathrm{deg} \leq \alpha<10 \mathrm{deg}$, respectively. Tables 1 and 2 summarize these values.

From the qualitative observations and tables 1 and 2, the following following conclusions can be drawn for the WLL:

- $C_{L} \times \alpha$ curves presented the same trends, regardless of the case: the discrepancies between the WLL for the two sources of numerical data increase with the increase in the AoA;

- for all the cases, the agreement between experimental and numerical was better for the case in which Star-CCM+ was used as the source of 2-D data. Still, the question about whether the time spent to generate a database must be answered. As observed on the verification procedure, modeling errors can be already in the order of $3.5 \%$, so the use of more accurate 2-D data must be justified by such an increase in accuracy;

- the conclusions about $C_{D}$ are unclear. Depending on $\alpha$ and on the wing geometry, the use of 2-D data-enhanced, was indifferent or diminished the accuracy in comparison to the experimental data. Such behavior might have happened due to the strong dependence of drag on Reynolds and the 3-D effects of the flow. Probably, the use of a more accurate database (in which $R e$ is taken as another input variable) could increase the results for $C_{D}$. 
Table 1 - Validation for the Wing Lifting-Line formulation. Lift Coefficient

\begin{tabular}{|c|c|c|c|c|c|c|}
\hline & Wing & & Elliptical & Crescent & Rectangular & 45-deg Sweep \\
\hline \multirow{6}{*}{$\alpha_{S}$} & \multirow{2}{*}{ Experimental } & $C_{L}$ & 0.2038 & 0.2038 & 0.12 & 0.121 \\
\hline & & $u_{C_{L}}$ & 0.0022 & 0.0022 & $\mathrm{~N} / \mathrm{A}$ & $\mathrm{N} / \mathrm{A}$ \\
\hline & \multirow{4}{*}{ Numerical } & $C_{L}$ & 0.2046 & 0.1994 & 0.16 & 0.120 \\
\hline & & $u_{C_{L}}$ & 0.0076 & 0.0065 & 0.01 & 0.004 \\
\hline & & $\mathrm{E}$ & 0.0008 & -0.0044 & 0.04 & -0.001 \\
\hline & & $u_{v}$ & 0.0079 & 0.0069 & 0.01 & 0.004 \\
\hline \multirow{6}{*}{$\alpha_{M}$} & \multirow{2}{*}{ Experimental } & $C_{L}$ & 0.5932 & 0.5932 & 0.56 & 0.456 \\
\hline & & $u_{C_{L}}$ & 0.0022 & 0.0022 & $\mathrm{~N} / \mathrm{A}$ & $\mathrm{N} / \mathrm{A}$ \\
\hline & \multirow{4}{*}{ Numerical } & $C_{L}$ & 0.5823 & 0.5675 & 0.60 & 0.456 \\
\hline & & $u_{C_{L}}$ & 0.0221 & 0.0204 & 0.02 & 0.017 \\
\hline & & $\mathrm{E}$ & -0.0109 & -0.0257 & 0.04 & 0.000 \\
\hline & & $u_{v}$ & 0.0222 & 0.0206 & 0.02 & 0.017 \\
\hline \multirow{6}{*}{$\alpha_{L}$} & \multirow{2}{*}{ Experimental } & $C_{L}$ & 0.7809 & 0.7778 & 1.04 & 0.559 \\
\hline & & $u_{C_{L}}$ & 0.0022 & 0.0022 & $\mathrm{~N} / \mathrm{A}$ & $\mathrm{N} / \mathrm{A}$ \\
\hline & \multirow{4}{*}{ Numerical } & $C_{L}$ & 0.7646 & 0.7454 & 1.05 & 0.575 \\
\hline & & $u_{C_{L}}$ & 0.0295 & 0.0241 & 0.04 & 0.021 \\
\hline & & $\mathrm{E}$ & -0.0163 & -0.0324 & 0.01 & 0.016 \\
\hline & & $u_{v}$ & 0.0295 & 0.0242 & 0.04 & 0.021 \\
\hline
\end{tabular}

Source: Author

Table 2 - Validation for the Wing Lifting-Line formulation. Drag Coefficient

\begin{tabular}{|c|c|c|c|c|c|c|}
\hline & Wing & & Elliptical & Crescent & Rectangular & 45-deg Sweep \\
\hline \multirow{6}{*}{$\alpha_{S}$} & \multirow{2}{*}{ Experimental } & $C_{D}$ & 0.01514 & 0.01514 & 0.011 & 0.0039 \\
\hline & & $u_{C_{D}}$ & 0.00050 & 0.00050 & $\mathrm{~N} / \mathrm{A}$ & $\mathrm{N} / \mathrm{A}$ \\
\hline & \multirow{4}{*}{ Numerical } & $C_{D}$ & 0.01535 & 0.01546 & 0.011 & 0.0074 \\
\hline & & $u_{C_{D}}$ & 0.00020 & 0.00018 & 0.000 & 0.0001 \\
\hline & & $\mathrm{E}$ & 0.00021 & 0.00032 & 0.000 & 0.0035 \\
\hline & & $u_{v}$ & 0.00054 & 0.00053 & 0.000 & 0.0001 \\
\hline \multirow{6}{*}{$\alpha_{M}$} & \multirow{2}{*}{ Experimental } & $C_{D}$ & 0.03213 & 0.03123 & 0.035 & 0.0385 \\
\hline & & $u_{C_{D}}$ & 0.00050 & 0.00050 & $\mathrm{~N} / \mathrm{A}$ & $\mathrm{N} / \mathrm{A}$ \\
\hline & \multirow{4}{*}{ Numerical } & $C_{D}$ & 0.03261 & 0.03525 & 0.037 & 0.0386 \\
\hline & & $u_{C_{D}}$ & 0.00163 & 0.00226 & 0.002 & 0.0016 \\
\hline & & $\mathrm{E}$ & 0.00048 & 0.00402 & 0.002 & 0.0001 \\
\hline & & $u_{v}$ & 0.00171 & 0.00231 & 0.002 & 0.0016 \\
\hline \multirow{6}{*}{$\alpha_{L}$} & \multirow{2}{*}{ Experimental } & $C_{D}$ & 0.04602 & 0.04582 & 0.097 & 0.0600 \\
\hline & & $u_{C_{D}}$ & 0.00050 & 0.00050 & $\mathrm{~N} / \mathrm{A}$ & $\mathrm{N} / \mathrm{A}$ \\
\hline & \multirow{4}{*}{ Numerical } & $C_{D}$ & 0.04759 & 0.04907 & 0.102 & 0.0581 \\
\hline & & $u_{C_{D}}$ & 0.00282 & 0.00256 & 0.006 & 0.0026 \\
\hline & & $\mathrm{E}$ & 0.00157 & 0.00325 & 0.005 & -0.0019 \\
\hline & & $u_{v}$ & 0.00286 & 0.00261 & 0.006 & 0.0026 \\
\hline
\end{tabular}

Source: Author 
- table 1 shows that - by taking $E=10 u_{C_{L}}$ as the adopted "much larger" criterion on equation (3.40), for both $\alpha_{S}$ and $\alpha_{L}, 75 \%$ of the data fall within noise level, according to equation (3.41), while this happens for $50 \%$ of the $\alpha_{M}$ data. However, no data shows evidence that the model could be improved, which is not expected, as the method should become worse as the angle of attack increases. The latter might have happened due to the large value of the validation uncertainty.

- a similar trend is observed on table 2, with the only differences that, for $\alpha_{M}$ the percentage is also $75 \%$, and for $\alpha_{S}$, the criterion of equation (3.40) is met for the 45-deg sweptback wing. This is expected, as it has already been previously discussed.

- as a preliminary analysis tool, the proposed WLL seems suitable, since direct comparison showed $C_{L}$ errors no higher than $10 \%$. Moreover, if the TFT is decided to be used, it is wise to include a constant $C_{D_{0}}$ value as an estimate of the viscous drag contribution on the overall drag.

\subsection{Propeller Lifting-line}

Since in the propeller formulation the wake is discretized into finite numbers of straight vortex segments as to approximate the geometry of helical horseshoes, the converge behavior of this discretization must be assessed as well; hence, only with the appropriate wake discretization, the blade discretization can be evaluated. After that, only two different cases are used in the blade verification process: one for the linear, and the other for the nonlinear scheme, as in the PLL case the evaluation of isolated geometric characteristics is not as important as it was for the WLL - either a pitch, rake, or skew distribution alone is sufficient to already make the quarter-chord line become out of a unique plane. Moreover, also for the PLL, the choice between two clusterings previously discussed will be assessed; they are depicted in figure 47.

\subsubsection{Code and Solution Verification}

\subsubsection{Wake model}

The proposed wake model is refined by two interdependent ways: (i) increasing the number of straight vortices per single loop and (ii) increasing the number of single loops $N_{h}$, as illustrated in figure 48 .

The verification starts with the LVD to determine a minimum number of straight segments that make the velocity induced by the discrete single helical loop be in the asymptotic region (of a continuous helical single loop); although no analytic value exists for such geometry, a rigorous analytic solution is dismissed as the discretization graphically 
Figure 47 - The two clusterings evaluated: equidistant and cosine discretizations. Propeller case.

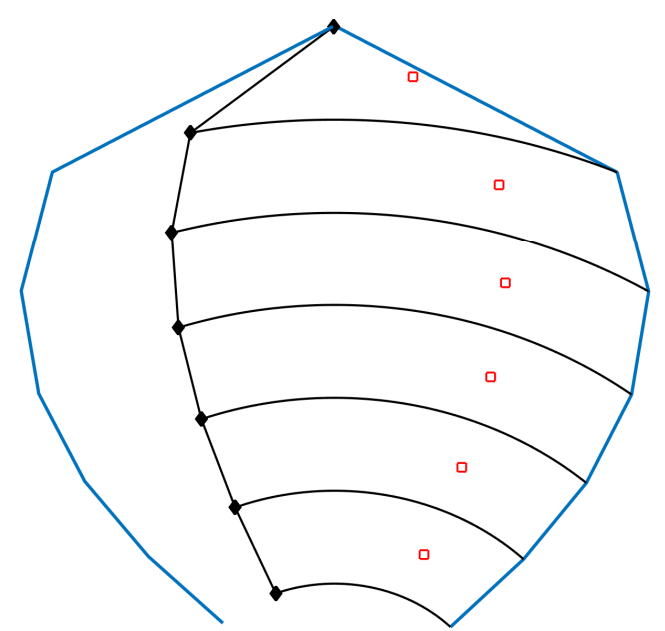

Equidistant

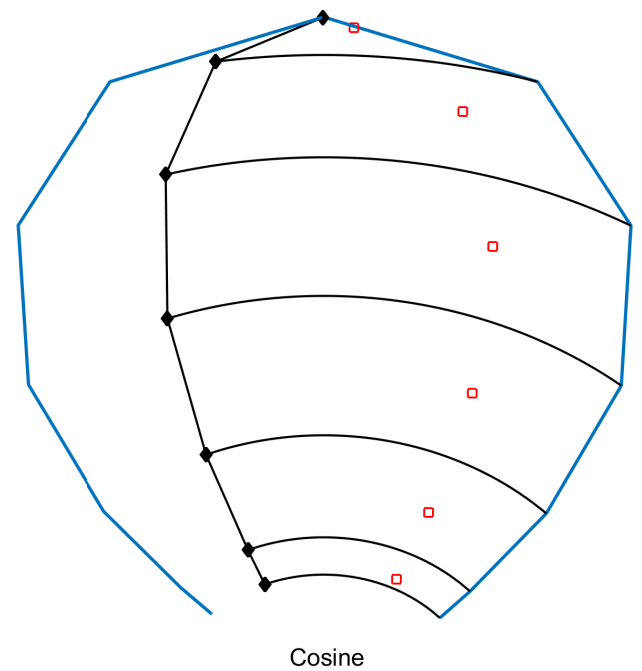

Source: Author

Figure 48 - Scenario for the Loop-Vortex Density (LVD) (left) and $N_{H}$ (right) convergence analyses.

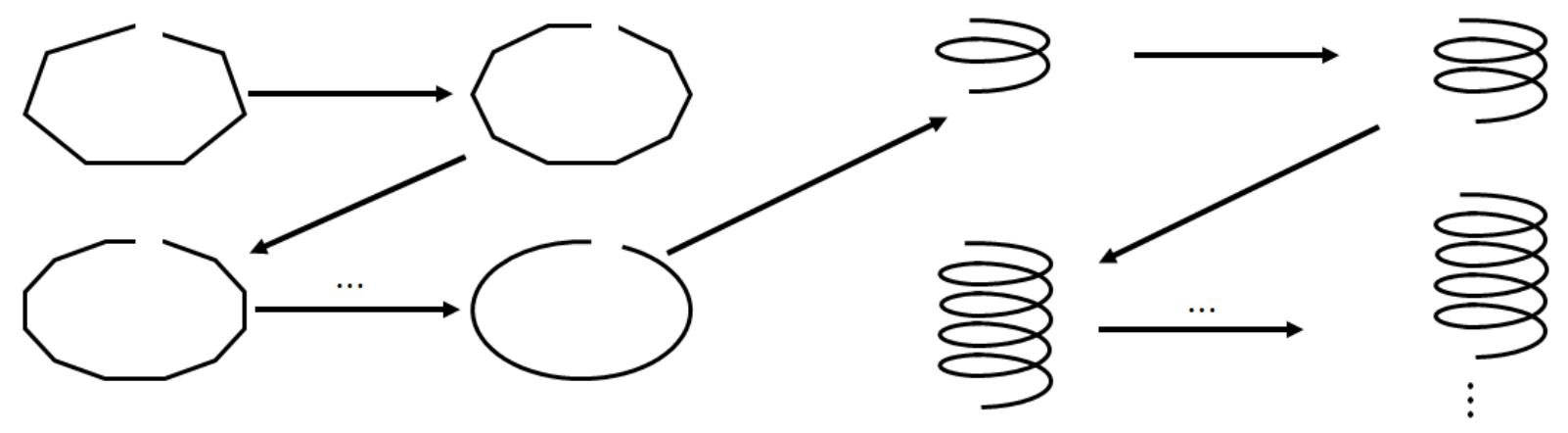

Source: Author

represents a numerical integration of the velocity $d \vec{V}$ induced by all the $N_{s}$ loop segments of infinitesimal length as in equation (2.18), i.e.:

$$
\vec{V} \int_{\text {loop }} d \vec{V} \approx \sum_{k_{j}=1}^{N_{S}} \vec{V}_{V S, i j, k_{j} k_{j+1}}^{b_{i} b_{j}}
$$

To evaluate such numerical integration, the chosen pitch angle $\beta$ was approximately $17 \mathrm{deg}$ and the loop radius $r_{\text {loop }}=0.150 \mathrm{~m}$. The probe at which the magnitude of the velocity was calculated as at the radial center on the loop; finally, the poorest wake has 18 straight segments, and the finest 9216, with a consecutive increase in the number of elements by a ratio of $2 . \delta l_{i}$ was considered the length of each segment, such that, from 
equation (3.14):

$$
h_{L V D}=\frac{\sum_{i=1}^{N_{s}} \delta l_{i}}{N_{s}}=\delta l_{i}
$$

as all the segments have equal value on the present analysis. Figure 49 shows the convergence of the magnitude of the induced velocity $V_{L V D}$ as a percentage of the extrapolated value $V_{L V D, f}$; an order of convergence $p_{L V D}$ of approximately 2 is observed along with an uncertainty $u_{L V D}=2.3895 \times 10^{-7}$, figures that corroborate the appropriateness of the discretization to represent a single loop. However, representing a single loop by almost ten thousand elements is rather exaggerated. The zoom in the converged regions shows that, for $\frac{h}{h_{f}}<35$ (i.e., $L V D \geq 288$ ), the relative error from the extrapolated value is already under $0.01 \%$, and for this particular value, $u_{L V D}$ is about $5.8982 \times 10^{-4}$; the velocity induced by a single helical loop can be reasonably represented by $N_{s} \approx 288$ (or even lower if the associated uncertainty does not increase drastically).

Figure 49 - Convergence of the magnitude of the induced velocity at the radial center of the loop as LVD is increased.
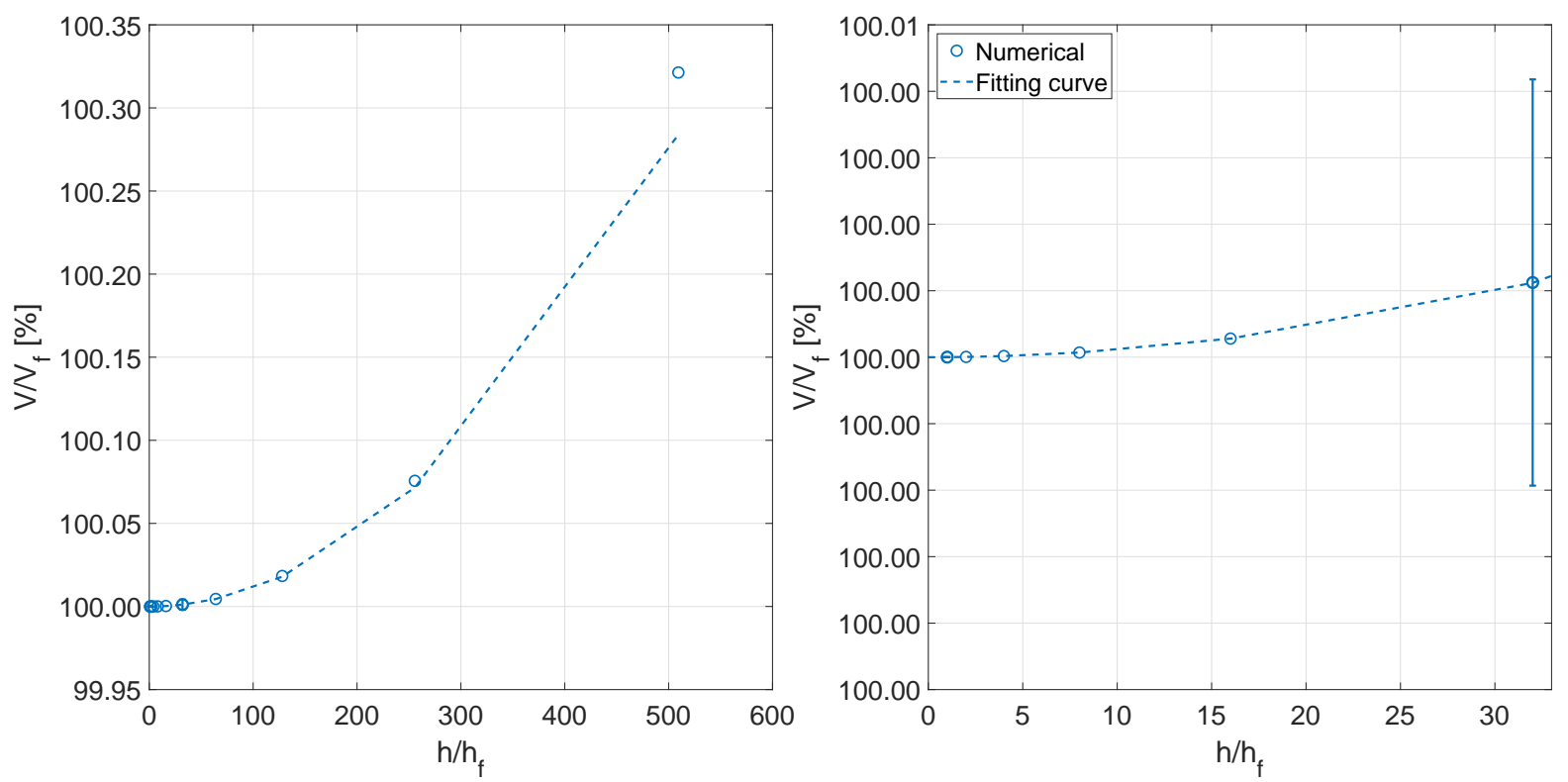

Source: Author

Keeping LVD as the finest value of the previous analysis, the number of single loops is increased from 1 to 1024 by a ratio of 2 to assess the adequate number of loops that make the velocity induced as a function of $N_{H}$ be in the asymptotic limit of a semi-infinite helical loop. For this particular case, $h$ is defined as in equation (4.9), because only the total length of the horseshoe is the effective length that changes (and such length is not a 
representative discretization length).

$$
h_{N h}=\frac{1}{N_{h}}
$$

The observed $p_{N h}$ is approximately 2 and the associated uncertainty is $u_{N_{H}}=$ $2.9792 \times 10^{-6}$, which indicates adequate convergence and numerical uncertainty for the proposed approach. However, as in the case of LVD, the use of 1024 loops is unfeasible in terms of calculations. Reconsidering the analysis for a more representative number, such as 8 loops, the relative error from the extrapolated value is about $0.2 \%$, and the associated uncertainty $u_{N_{H}}=2.3094 \times 10^{-4}$. The four first $N_{H}$ are not in an asymptotic region, so the respective order of convergence gets polluted and the uncertainty analysis must be performed through the nonlinear least-square method.

Figure 50 - Asymptotic behavior of the induced velocity as $N_{H}$ is increased.
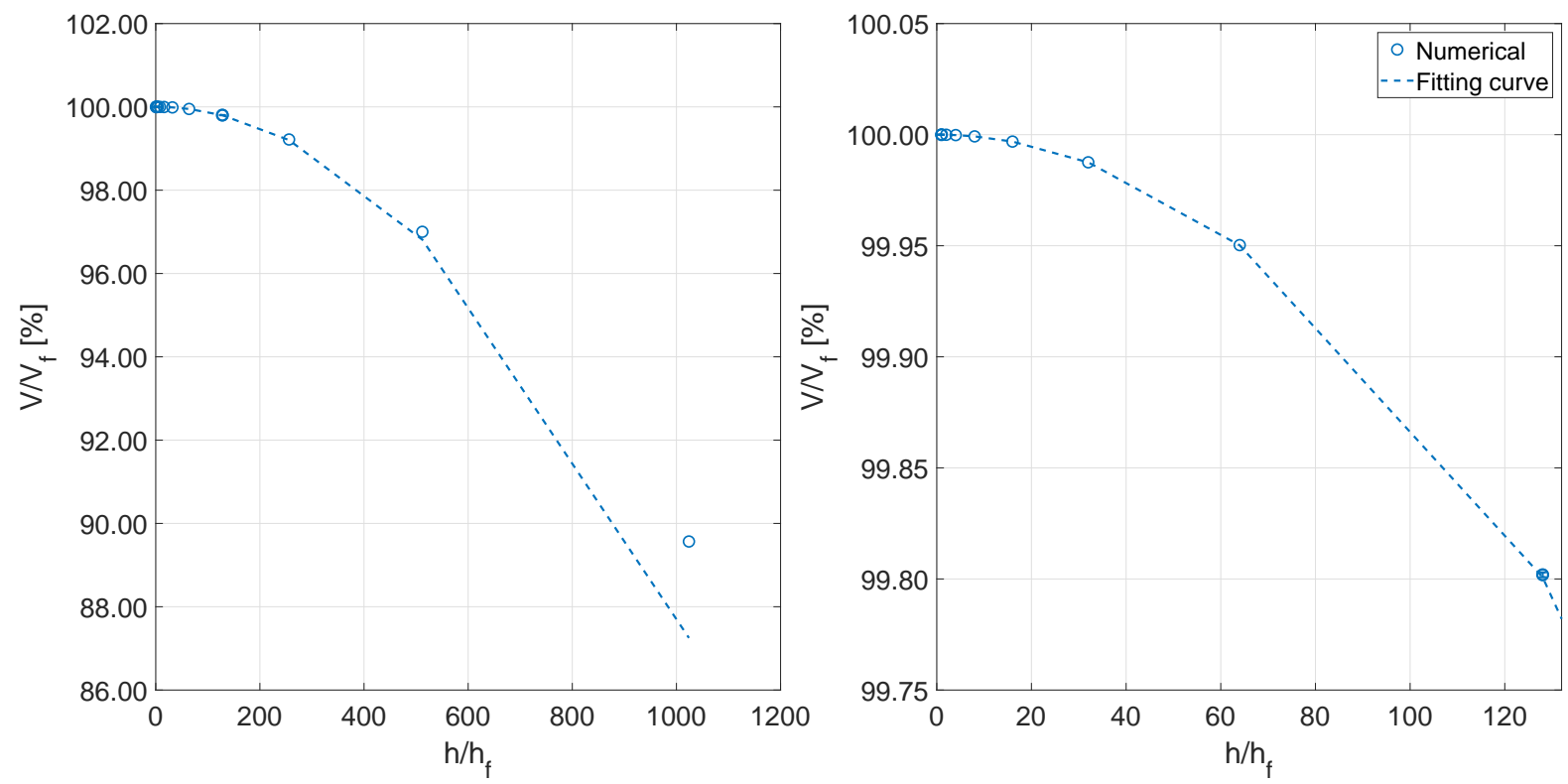

Source: Author

In order to compare the outputs of the combined discretization, the induced velocity of an entire discretized semi-infinite vortex is compared against analytic results; JR 1957 derived analytic expressions - known as Wrench formulas - for the velocities induced by HHSV s having a common radius of revolution $R$ and that are placed at a radial distance $R$ from the origin, as depicted on figure 51. These expressions are given in terms of existing Bessel functions and depend on the number of blades and the wake pitch angle (KERWIN; HADLER, 2010). Thus, identifying $\vec{V}_{I n d}=\left[v_{t} v_{s} v_{a}\right]$, for $R<R_{B V}$ : 


$$
\begin{gathered}
v_{a}(R)=\frac{Z}{4 \pi R}\left(y-2 Z R_{B V} F_{1}\right) \\
v_{t}(R)=\frac{Z^{2}}{2 \pi R} y_{0} F_{1}
\end{gathered}
$$

while for $R>R_{B V}$ :

$$
\begin{gathered}
v_{a}(R)=-\frac{Z^{2}}{2 \pi R} y y_{0} F_{2} \\
v_{t}(R)=\frac{Z}{4 \pi R}\left(1+2 Z y_{0} F_{2}\right)
\end{gathered}
$$

in which:

$$
\begin{aligned}
& F_{1} \approx-\frac{1}{2 Z y_{0}}\left(\frac{1+y_{0}^{2}}{1+y^{2}}\right)^{0.25}\left\{\frac{1}{U^{-1}-1}+\frac{1}{24 Z}\left[\frac{9 y_{0}^{2}+2}{\left(1+y_{0}^{2}\right)^{1.5}}+\frac{3 y^{2}-2}{\left(1+y^{2}\right)^{1.5}}\right] \ln \left(1+\frac{1}{U^{-1}-1}\right)\right\} \\
& F_{2} \approx-\frac{1}{2 Z y_{0}}\left(\frac{1+y_{0}^{2}}{1+y^{2}}\right)^{0.25}\left\{\frac{1}{U-1}-\frac{1}{24 Z}\left[\frac{9 y_{0}^{2}+2}{\left(1+y_{0}^{2}\right)^{1.5}}+\frac{3 y^{2}-2}{\left(1+y^{2}\right)^{1.5}}\right] \ln \left(1+\frac{1}{U-1}\right)\right\}
\end{aligned}
$$

and:

$$
\begin{gathered}
U=\left\{\frac{y_{0}\left(\sqrt{1+y^{2}}-1\right)}{y\left(\sqrt{1+y_{0}^{2}}-1\right)} \exp \left(\sqrt{1+y^{2}}-\sqrt{1+y_{0}^{2}}\right)\right\}^{Z} \\
y=\frac{R}{R_{B V} \tan \beta_{w}} \\
y_{0}=\frac{1}{\tan \beta_{w}}
\end{gathered}
$$

Figure 52 presents the behavior of the axial and tangential induced velocities normalized by the inflow, with $Z$ fixed to 5 and the pitch angle varying from 10 deg to 60 deg. With $N_{H}=8$ and LVD $=288$, the numerical wake graphically seems to adequately reproduce the analytic results, as both values practically overlap throughout the entire $\frac{R}{R_{B V}}$ range.

Now, with a fixed $\beta$ of $30 \mathrm{deg}$ and varying $Z$ from 2 to 7 , the results of figure 53 are obtained, and they seem similar as to before; the results, therefore, indicate a representative proposed wake model.

Once the limits have been established for the wake representation, verification is carried for the blade discretization; the lack of analytic results for the propeller hydrodynamics, however, does not permit the assessment of an order of magnitude of the modeling 
Figure 51 - Scenario used for the comparison between the numerical velocity induced by the helical model and the analytic values from the Wrench formulas.

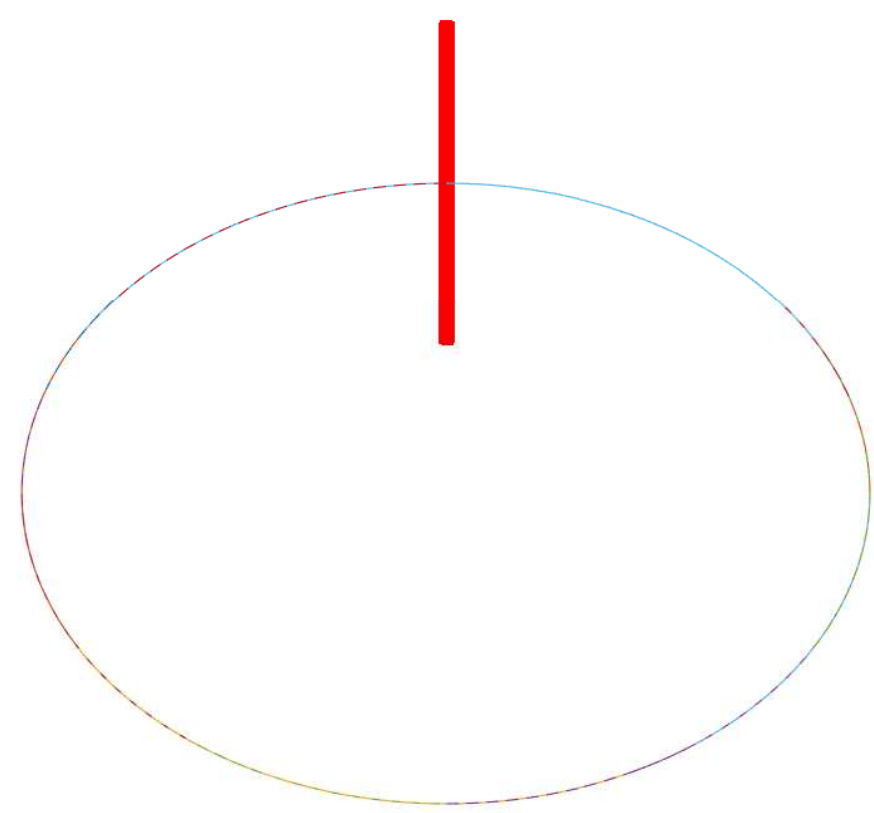

Source: Author

Figure 52 - Comparison between numerical results and the Wrench formulas for $Z=5$ and several $\beta$.
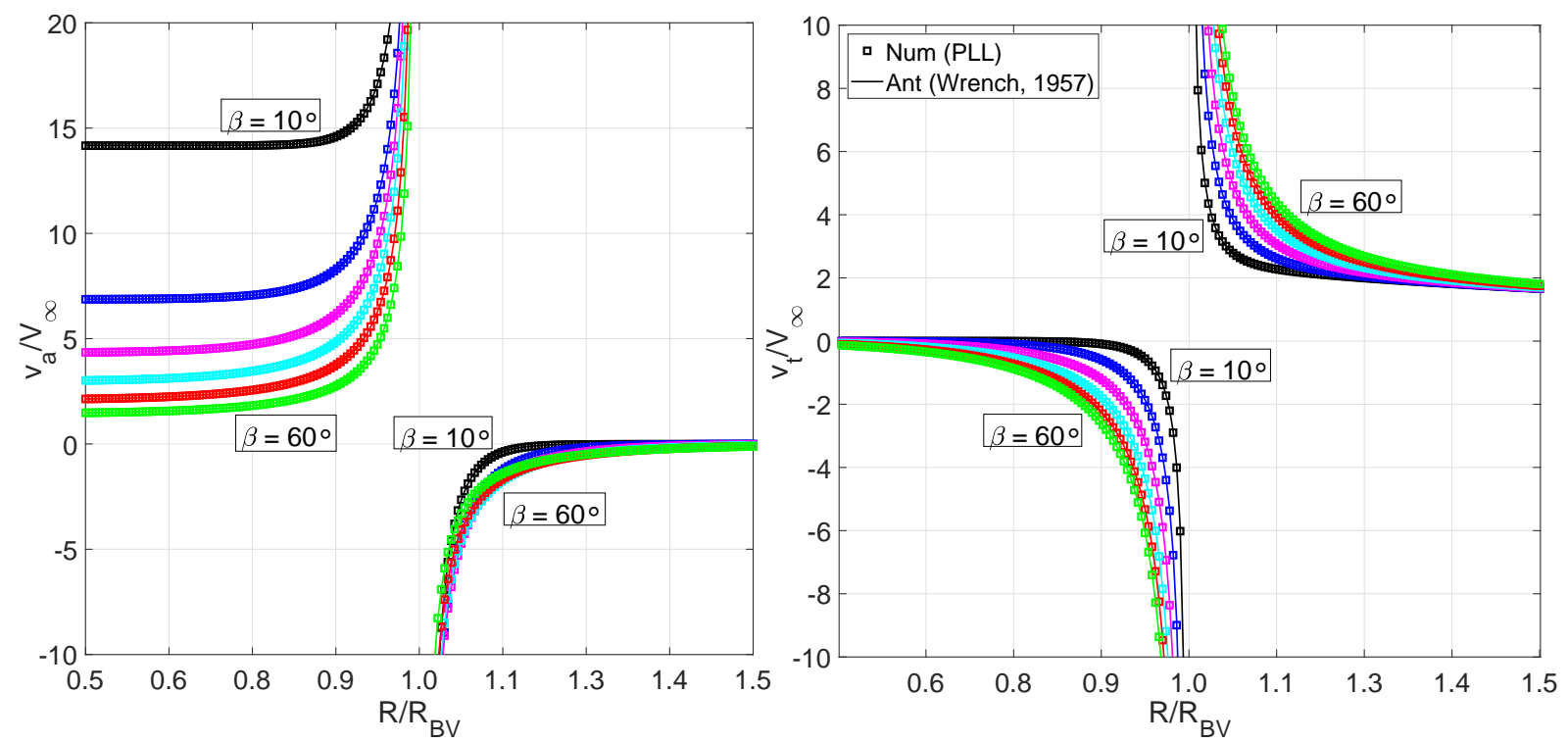

Source: Author

error. Although that is not the scope of such work, the development of manufactured solutions would have been a more adequate approach. As previously mentioned, only two 
Figure 53 - Comparison between numerical results and the Wrench formulas for $\beta=30$ deg and several $Z$.
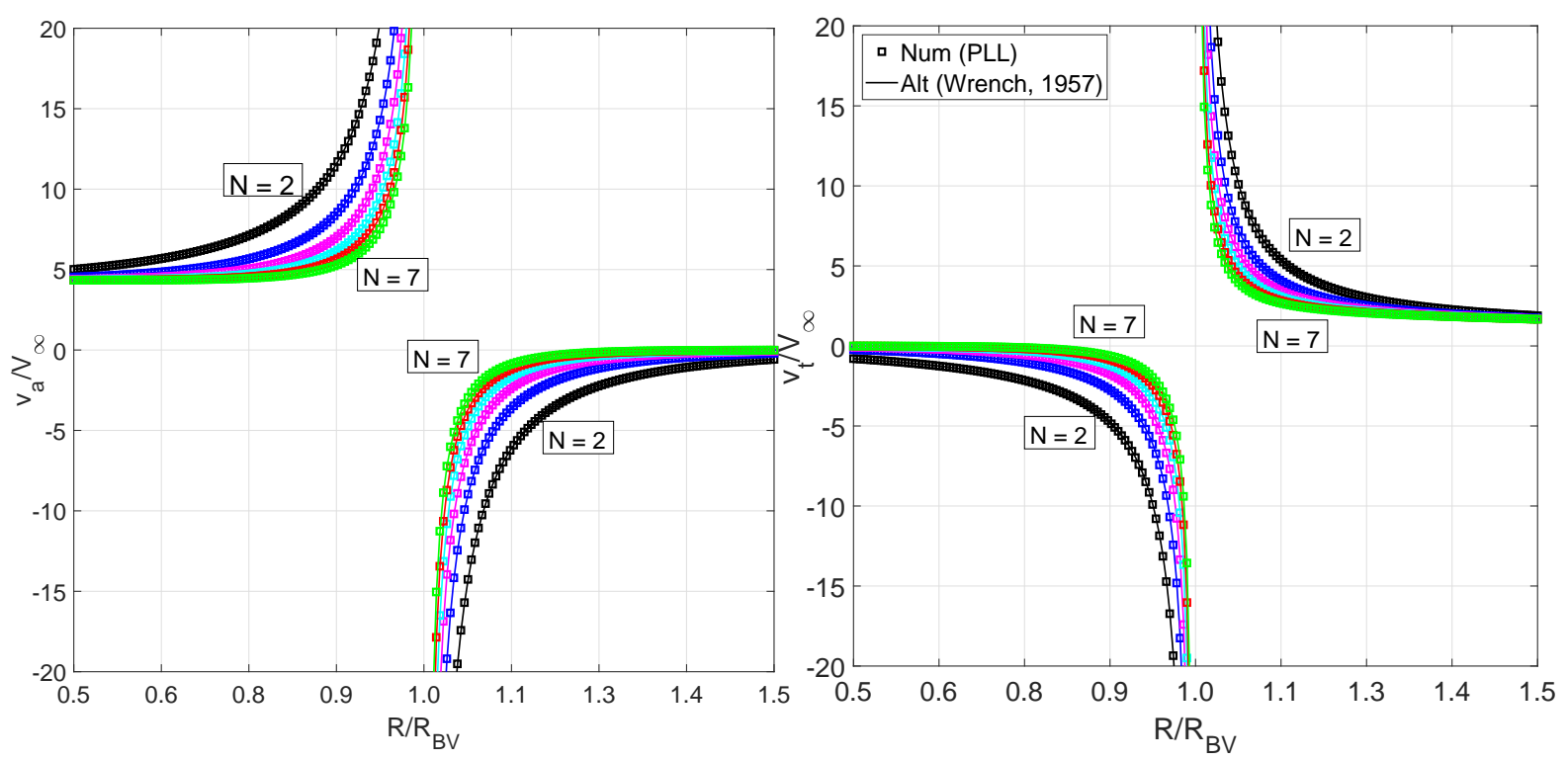

Source: Author

cases are tested to assess the linear and nonlinear schemes of the novel formulation.

\subsubsection{DTMB 4119 Propeller}

Near the 1970s, considerable improvements have been made in propeller design procedures, as the methods developed by KERWIN (1961) and others permitted the used of LST methods throughout the design procedure. Based on one of these methods, the David Taylor Model Basin (DTMB) has developed a series of six three-bladed propellers, named P41XX propellers, that varied from one another in terms of EAR, camber distribution, and skew. The propellers have been open-water and cavitation tested, and the results reported (DENNY, 1968); they yielded information concerning the validity of the LST on the treatment of the three aforementioned variables.

Perhaps the most extensively studied of these propellers has been the DTMB P4119 (JESSUP, 1989; SANCHEZ-CAJA, 1998; EPPS, 2017), a free-running propeller with a nearly ideal circulation distribution, fair blades, and moderate thickness, and whose discretization was depicted on figure 20; for this study, each blade has been discretized similarly to a wing semi-span, i.e., with $\mathrm{N}$ varying from 28 to 904 and using both equidistant and cosine discretizations, while $K_{T}$ was taken as the figure of merit.

Figure 54 presents the convergence trends for both discretizations as functions of the representative grid size normalized by the finest grid. As in the WLL case, the equidistant distribution did not present a satisfactorys observed convergence rate $\left(p_{E}=0.9348\right)$, while 
the cosine clustering presented an observed $p_{C}$ near the expected one, in addition to having comparably negligible variations of $K_{T}$ as $h$ decreases. Contrary to what happens in the WLL case, the extrapolated values are not the same on both dicretizations ( $K_{T, E \text {,ext }}$ differs from $K_{T, C, e x t}$ by about $1 \times 10^{-5}$ ). The trends for the uncercertainties, on the other hand, remain the same: $u_{h E}=8.3989 \times 10^{-5}$, while $u_{h C}=3.0547 \times 10^{-8}$.

Figure $54-K_{T}$ versus normalized $h$ for solution verification. DTMB P4119, linear scheme.
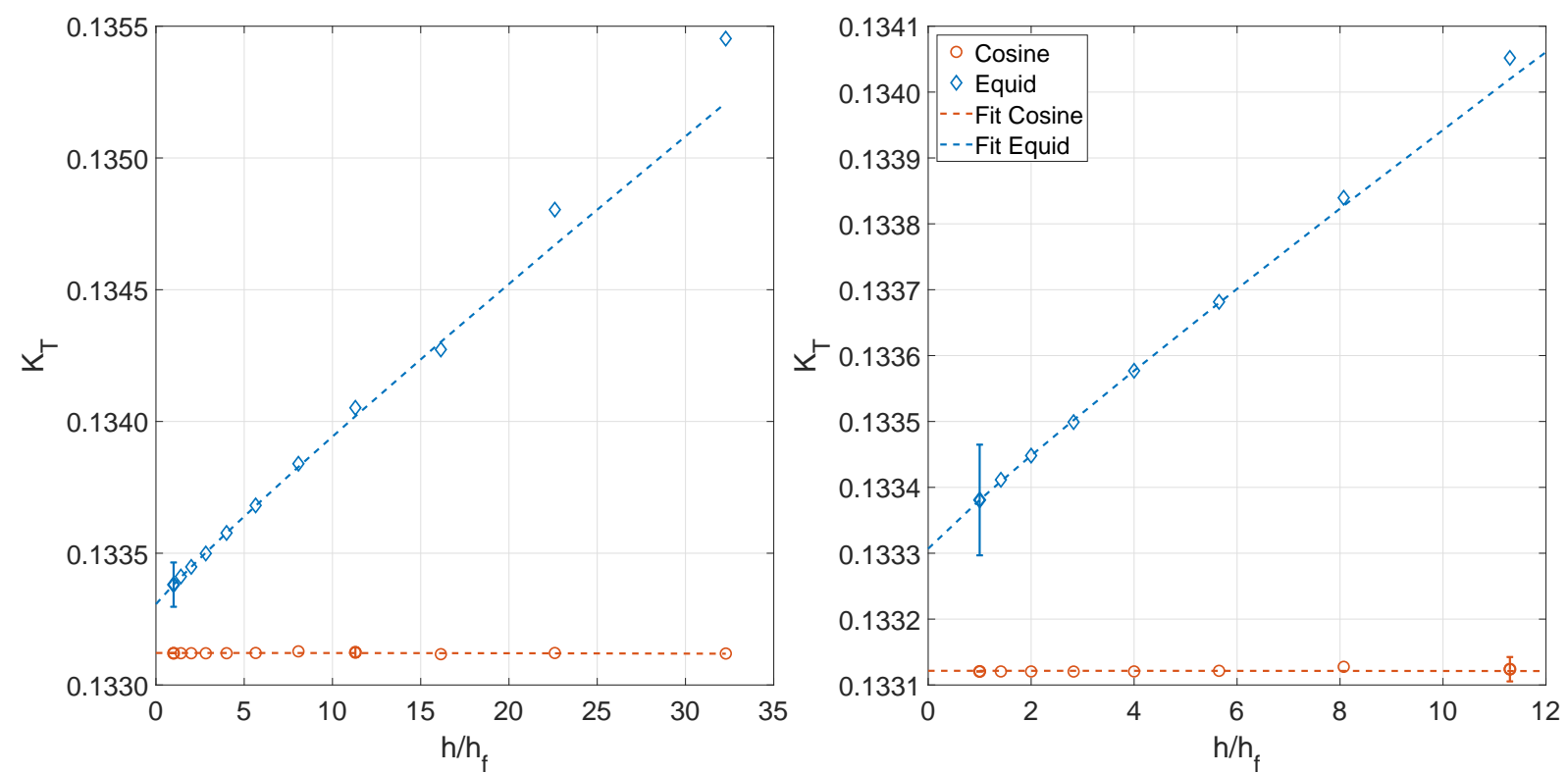

Source:Author

For the assessment of the nonlinear scheme, the source of 2-D data was from the expressions proposed by (BROCKETT, 1966) - equations (2.36) - as the only interest is the assessment of the convergence trends of the PLL iterative scheme as a function of grid refinement. Those presented in figure 55 .

As in the linear scheme, the equidistant distribution presented an observed rate of convergence lower than expected $\left(p_{E}=0.9265\right)$, whereas the cosine discretization had an observed $p_{C}$ close to the theoretical value. The $K_{T}$ extrapolated values differed by approximately $3 \times 10^{-4}$, and the uncertainties were $u_{h E}=8.5265 \times 10^{-5}$ and $u_{h C}=$ $2.1396 \times 10^{-8}$. Therefore, both linear and nonlinear formulations do not converge adequately for the equidistant discretization, while they do converge quite satisfactorily for the cosine discretization. It also must be pointed out that the results presented are worth attention as they imply that the proposed PLL formulation adequately converges for nonstraight BV lines.

\subsubsection{Conclusions about Code and Solution Verifications}

The code and solution verifications for the PLL lead to the following conclusions: 
Figure $55-K_{T}$ versus normalized $h$ for solution verification. DTMB P4119, Noninear scheme.
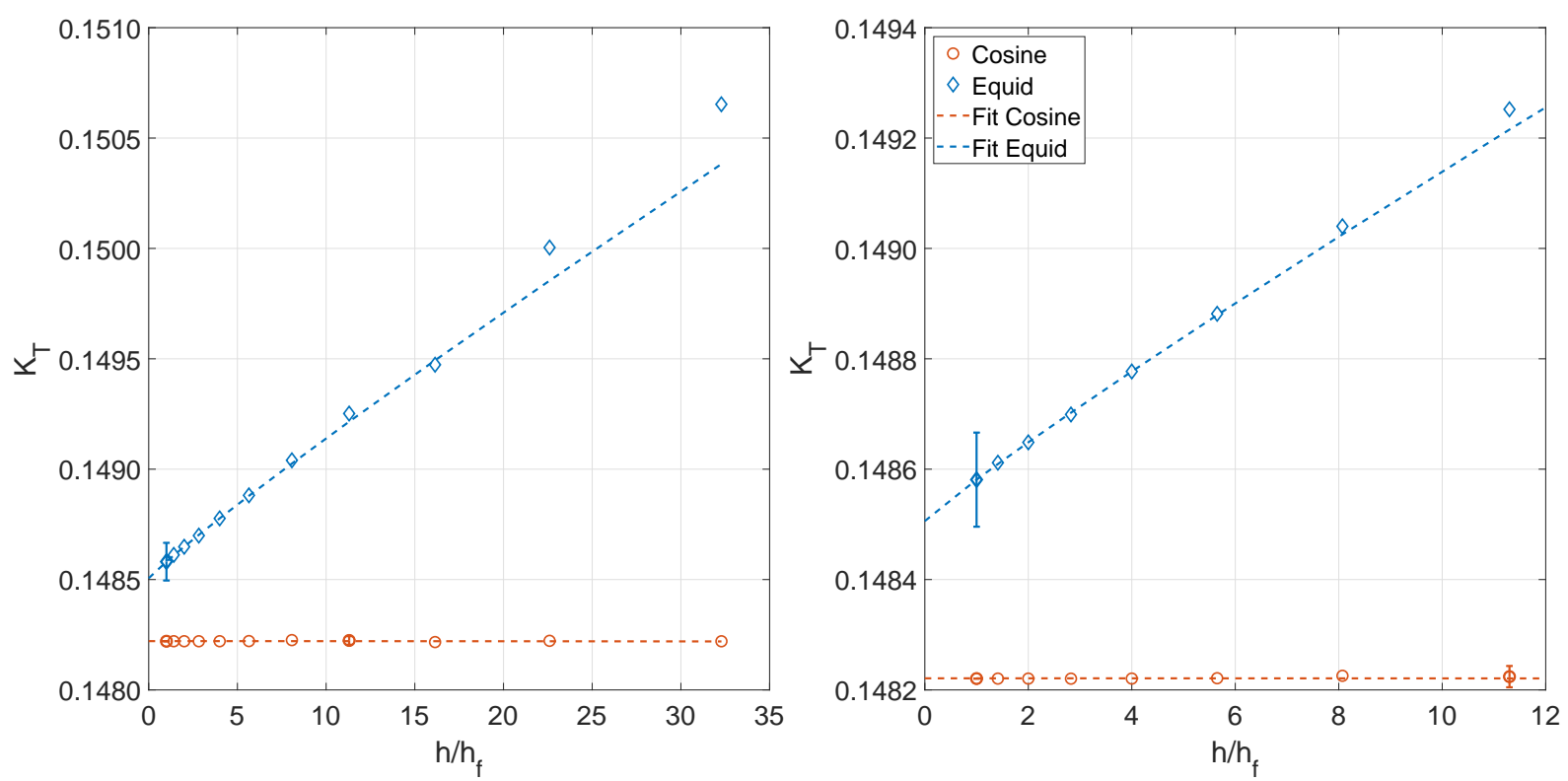

Source:Author

- assessment of the proposed wake model showed adequate convergence rates for the induced velocities of both interdependent discretizations, with relatively low induced velocity differences for reasonably coarse discretizations. The price to pay for such coarsening, however, is the increase in the numerical uncertainty;

- the accuracy of the wake model is corroborated by graphical comparison with the Wrench Formulas - equations (4.10) and (4.11) ;

- both linear and nonlinear schemes presented similar results in comparison to each other and also in comparison with the WLL: again the cosine clustering is the most appropriate, as the $p_{C} s$ obtained are always close to the theoretical one, while with the equidistant clustering $p_{E} s$ are lower, and according to the standard, the equidistant discretization probably has not been carried to a sufficient level;

- the inclusion of a source of 2-D data and (at least some degree) of sweep and dihedral angles do not seem to 'pollute' the numerical solution, meaning that the discretization uncertainties do not increase due them;

- the cosine discretization presented uncertainties in the order of magnitude of $O\left(u_{h C}\right) \approx 1 \times 10^{-8}$ while the cosine discretization presented uncertainties in the order of $O\left(u_{h E}\right) \approx 1 \times 10^{-5}$;

- the proposed formulation is capable of grid-converging, on both linear and nonlinear schemes, problems whose BV is not straight, which is a clue about the capability of 
the PLL to simulate raked and skewed propellers and to include more accurate 2-D data. The accuracy of the method will be assessed through the validation.

\subsubsection{Validation}

After the grid convergence study has also been carried for the PLL, the formulation is compared against several data from the literature (JESSUP, 1989; BARKMANN, 2011; KATSUNO; DANTAS, 2017; Silva Jr et al., 2019). Given the same trends obtained as in the WLL, cosine clustering with $N=112$ was set for the numerical simulations; this combination of clustering and discretization level, as shown on the verification plots figures 54 and $55, \frac{h}{h_{f}} \approx 12$-, have an associated numerical uncertainty $u_{n}$ in the order of $1 \times 10^{-5}$; for this formulation, however, the largest source of discretization uncertainty is from the wake model: as the number of calculations for the induced velocities will depend on the discretization level of the wake as well, a very fine wake makes the problem CPU time-consuming. Therefore, to make the simulation time feasible, LVD was set to 288 , while $N_{H}$ set to 8 and the associated uncertainties are in the order of $1 \times 10^{-3}$ and $1 \times 10^{-4}$, respectively. Additionally, the uncertainties related to the source of 2-D has also been carried out to the calculation of both $u_{K_{T}}$ and $u_{K_{Q}}$ as in the wing formulation.

\subsubsection{DTMB 4119 Propeller}

The DTMB P4119 has also been used as a validation test due to its simplicity and availability of data. The blade outline is described in table 3 , while a drawing of the propeller is shown in figure 56 .

Figure 56 - Propeller drawing. DTMB P4119.

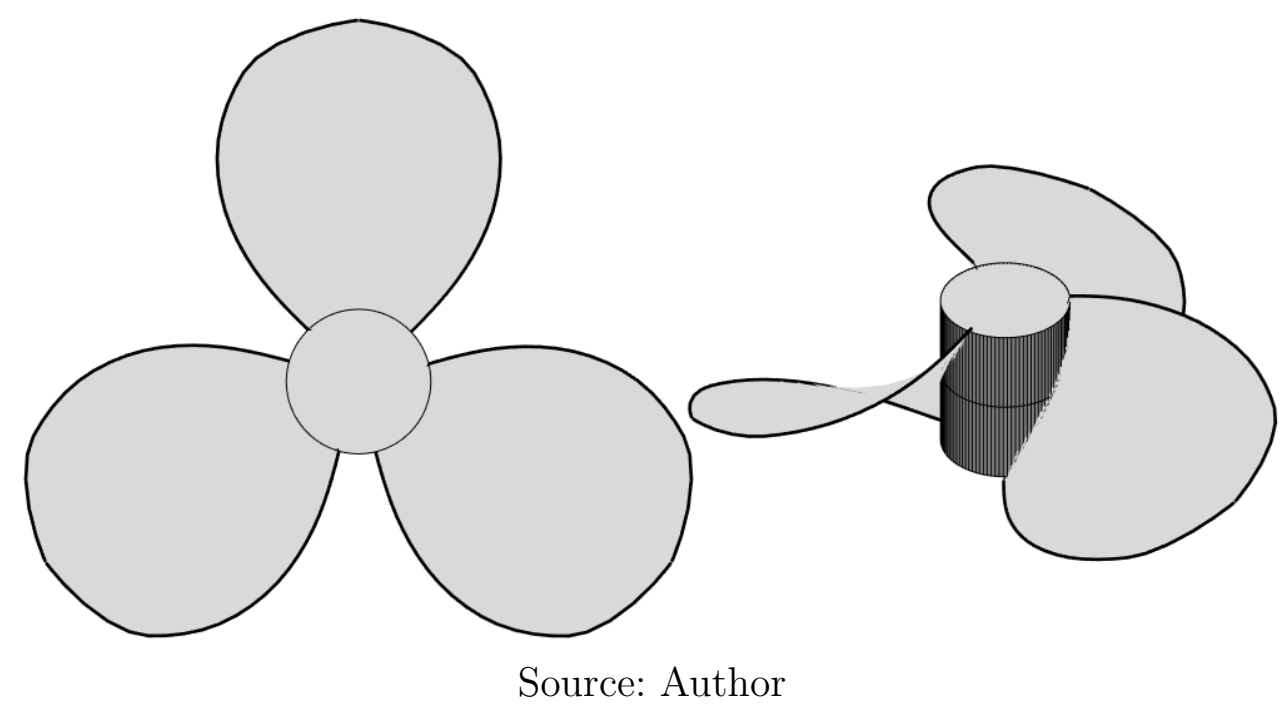

Open-water tests were carried at the DTMB for the previously mentioned family of propellers (DENNY, 1968). Their thrust and torque were determined over a range of 
Table 3 - Propeller characteristics and blade outline. DTMB P4119.

\begin{tabular}{|c|c|c|c|c|c|c|}
\hline $\begin{array}{l}\text { Numb } \\
\text { Hub } \\
\text { Expar } \\
\text { Sectio } \\
\text { Sectio } \\
\text { Desig }\end{array}$ & $\begin{array}{l}\text { r of bla } \\
\text { ameter } \\
\text { ded Are } \\
\text { mean } \\
\text { thickn } \\
\text { advanc }\end{array}$ & $\begin{array}{l}\text { es, Z: } 3 \\
\text { atio: } 0.2 \\
\text { Ratio: } \\
\text { ne: NAC } \\
\text { ss: NAC } \\
\text { coeffici }\end{array}$ & $\begin{array}{l}.606 \\
\mathrm{~A} \mathrm{a}=0 \\
\mathrm{~A} 66 \mathrm{TN} \\
\mathrm{nt}, \mathrm{J}=\end{array}$ & $\begin{array}{l}8 \\
\mathrm{~B} \bmod \\
.833\end{array}$ & & \\
\hline$\frac{r}{R}$ & $\frac{c}{D}$ & $\frac{P}{D}$ & $\frac{t_{0}}{c}$ & $\frac{f_{0}}{c}$ & $\theta_{S}$ & $\frac{z_{R}}{D}$ \\
\hline 0.200 & 0.3200 & 1.1050 & 0.2055 & 0.0143 & 0.0000 & 0.0000 \\
\hline 0.300 & 0.3625 & 1.1020 & 0.1553 & 0.0232 & 0.0000 & 0.0000 \\
\hline 0.400 & 0.4048 & 1.0980 & 0.1180 & 0.0230 & 0.0000 & 0.0000 \\
\hline 0.500 & 0.4392 & 1.0930 & 0.0902 & 0.0218 & 0.0000 & 0.0000 \\
\hline 0.600 & 0.4610 & 1.0880 & 0.0696 & 0.0207 & 0.0000 & 0.0000 \\
\hline 0.700 & 0.4622 & 1.0840 & 0.0542 & 0.0200 & 0.0000 & 0.0000 \\
\hline 0.800 & 0.4347 & 1.0810 & 0.0421 & 0.0197 & 0.0000 & 0.0000 \\
\hline 0.900 & 0.3613 & 1.0790 & 0.0332 & 0.0182 & 0.0000 & 0.0000 \\
\hline 0.950 & 0.2775 & 1.0770 & 0.0323 & 0.0163 & 0.0000 & 0.0000 \\
\hline 1.000 & 0.0020 & 1.0750 & 0.0316 & 0.0118 & 0.0000 & 0.0000 \\
\hline
\end{tabular}

Source: Author

advance coefficients from zero to 1.30, whereas the water speed and the revolutions of the propeller were kept sufficiently high to produce a minimum $R e_{\frac{r}{R}=0.70}$ of $5.0 \times 10^{5}$. The open-water performance was presented in the form of plots of $K_{T} \times J$ and $K_{Q} \times J$. The analysis of the accuracy of tests showed a negligible error in the advance coefficient. The uncertainties in $K_{T}$ and $K_{Q}$ are within $2.0 \%$ of the true value when considering both the accuracy and possible error readings of the dynamometer.

The lack of other sources of errors made only possible to calculate the overall numerical uncertainties based on the mesh (wake and blade discretizations) and the 2-D input data uncertainties. Figure 57 presents several curves for $K_{T}$ and $K_{Q}$ as function of $J$.

Comparison among numerical data indicates that for $K_{T} \times J$ the PLL presents very similar results when either the experimental expressions of Brockett or Star-CCM+ is used as source of 2-D data; both are almost equally far from the numerical results that used the TFT assumption and the absolute differences remain almost the same along the entire $J$ range. Between them, the differences are more evident at the lowest and highest $J$. In terms of $K_{Q} \times J$, as $J$ increases, so the difference between the PLL-TFT and the other two numerical results.

For the Star-CCM+ and Brockett-based PLL results, the same trends can be observed when compared to the experimental data: For $K_{T}$, at low $J$ the agreement is relatively far, while for $J$ close to the design advance coefficient and higher, the agreement 
is more satisfactory. For $K_{Q}$, the inclusion of foil data does not seem to contribute to the results, as all the curves presented similar (and relatively satisfying) results and for most of $J$ the absolute differences stay almost invariant.

Figure $57-K_{T}$ and $K_{Q}$ versus $J$. DTMB P4119.
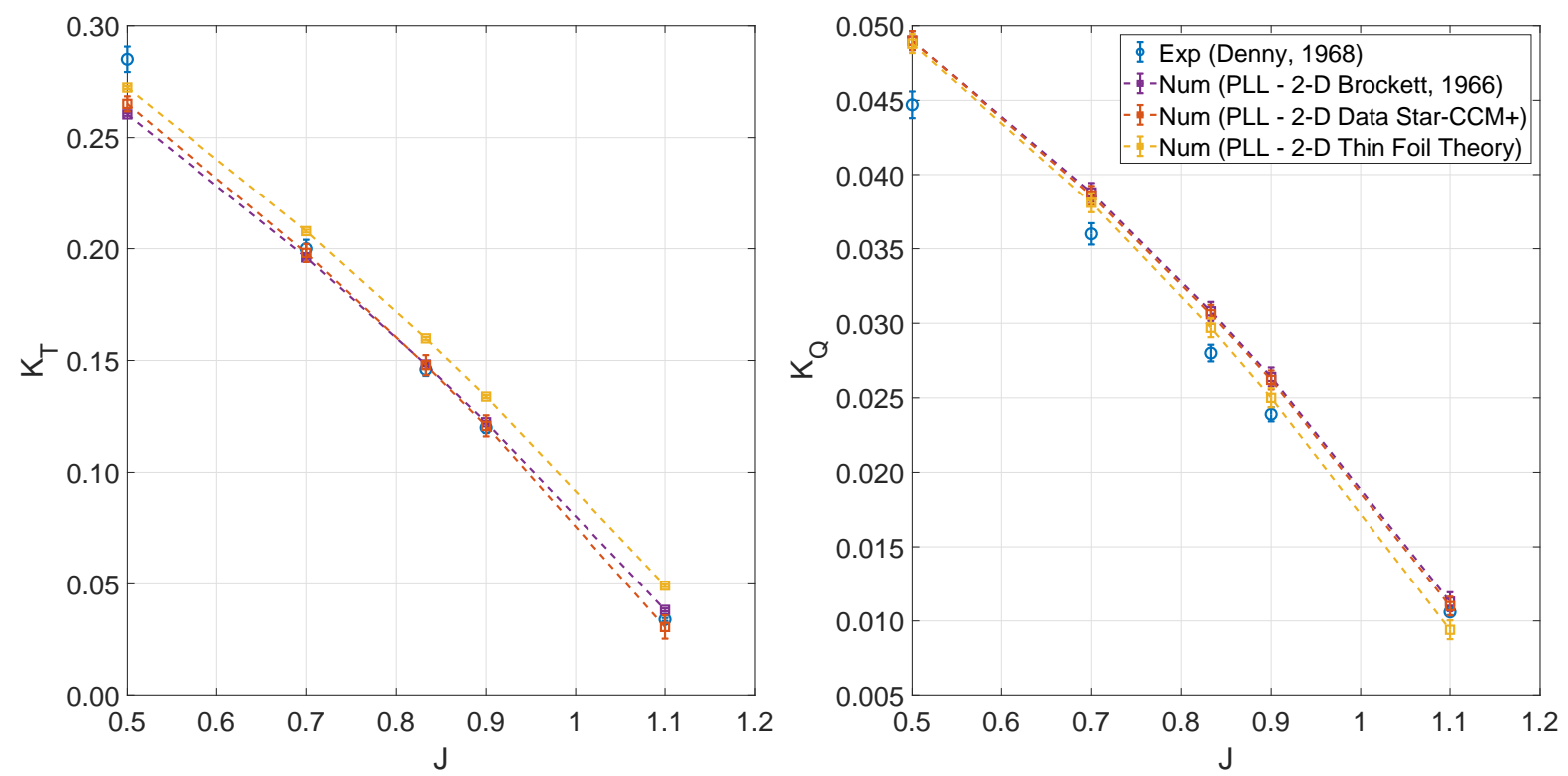

Source: Author

For $K_{T}$, one can expect the increase in the discrepancy as $J$ decreases, since the propeller starts departing from the moderately loaded condition. This departure can be quantitatively observed in figure 58, which presents the ratio of the induced velocities by the advance or rotational velocities as a function of $J$ for $\frac{r}{R} \approx 0.7$, and also the dependence of the thrust loading coefficient $C_{T}$ on $J$. For $J=0.5$, the axial induced velocity is already in the order of the advance velocity, a condition in which the moderately loaded assumption is no longer valid; this is corroborated by the high $C_{T}$. For $K_{Q}$, both values of 0.0085 and $C_{c}$ data from Star-CCM+ seem somewhat overestimated, as both induced and viscous torque of the two 2-D numerical-based PLLs sum up to the solely induced torque of the PLL-TFT case - i.e., for the Brockett and Star-CCM+ fed PLL, the $K_{Q \text {,ind }}$ is lower than the TFT based counterpart, but the sum $K_{Q, i n d}+K_{Q, 0}$ is approximately equal; lower (or more adequate) $C_{c}$ would probably reduce $K_{Q, 0}$ and, consequently the overall torque.

\subsubsection{DTMB 4133 Propeller}

DTMB P4133 planform is similar to P4119 but with doubled chord length sections, making it a propeller with large EAR or, equivalently, low Aspect Ratio (AR); such characteristic is interesting to be assessed as the classic LLT is limited to wings of large (higher than 4) AR (KATZ; PLOTKIN, 2001). It also has an almost constant pitch 
Figure $58-\frac{v_{a}}{V_{\infty}} \times J$ and $\frac{v_{t}}{V_{t}} \times J$ for $\frac{r}{R}=0.7$ and $C_{T}$ versus $J$. DTMB P4119.
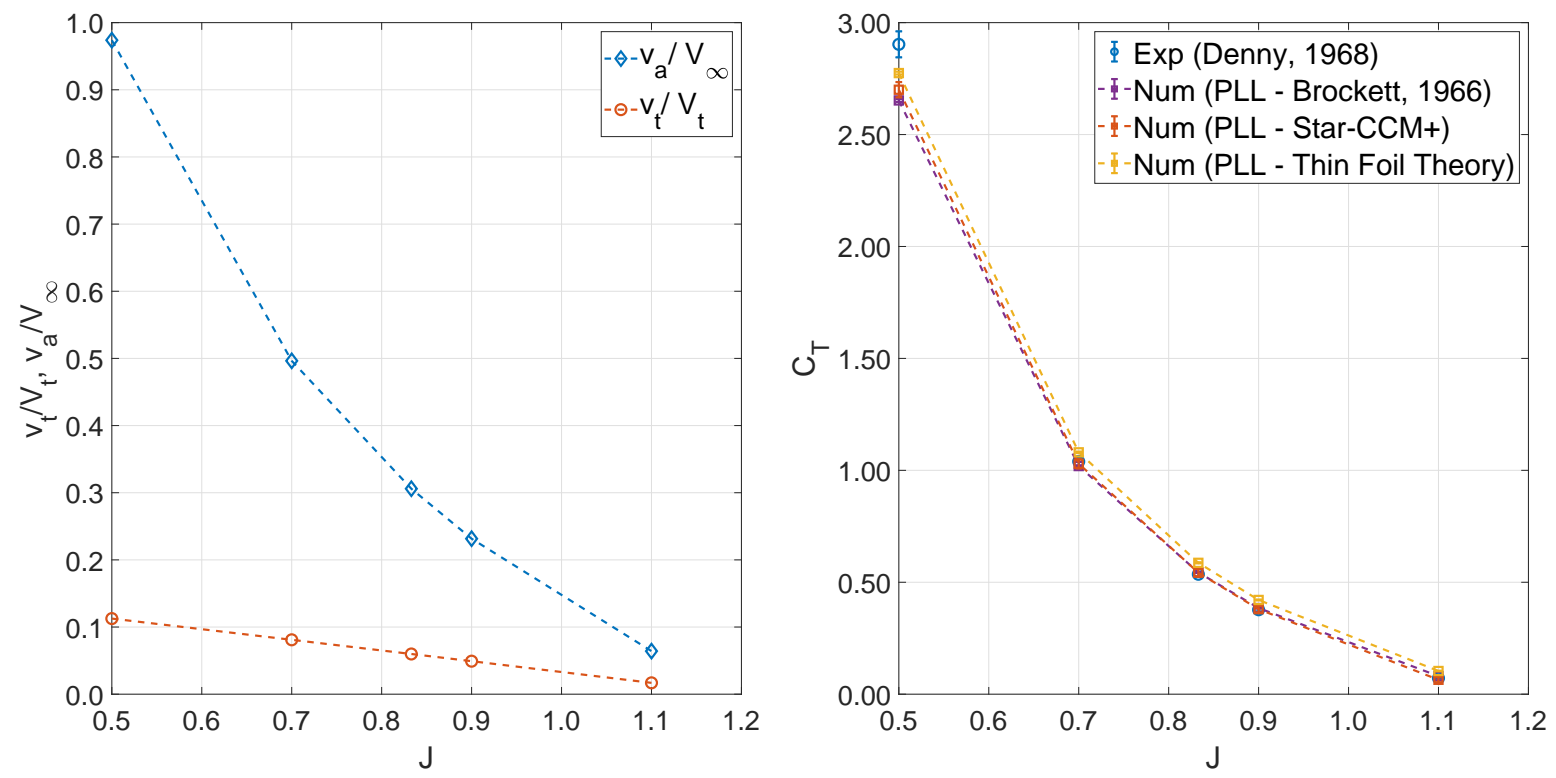

Source: Author

distribution due to its thinner blade sections. The blade outline is described in table 4 , while a drawing of the propeller is presented in figure 59 .

Figure 59 - Propeller drawing. DTMB P4133.

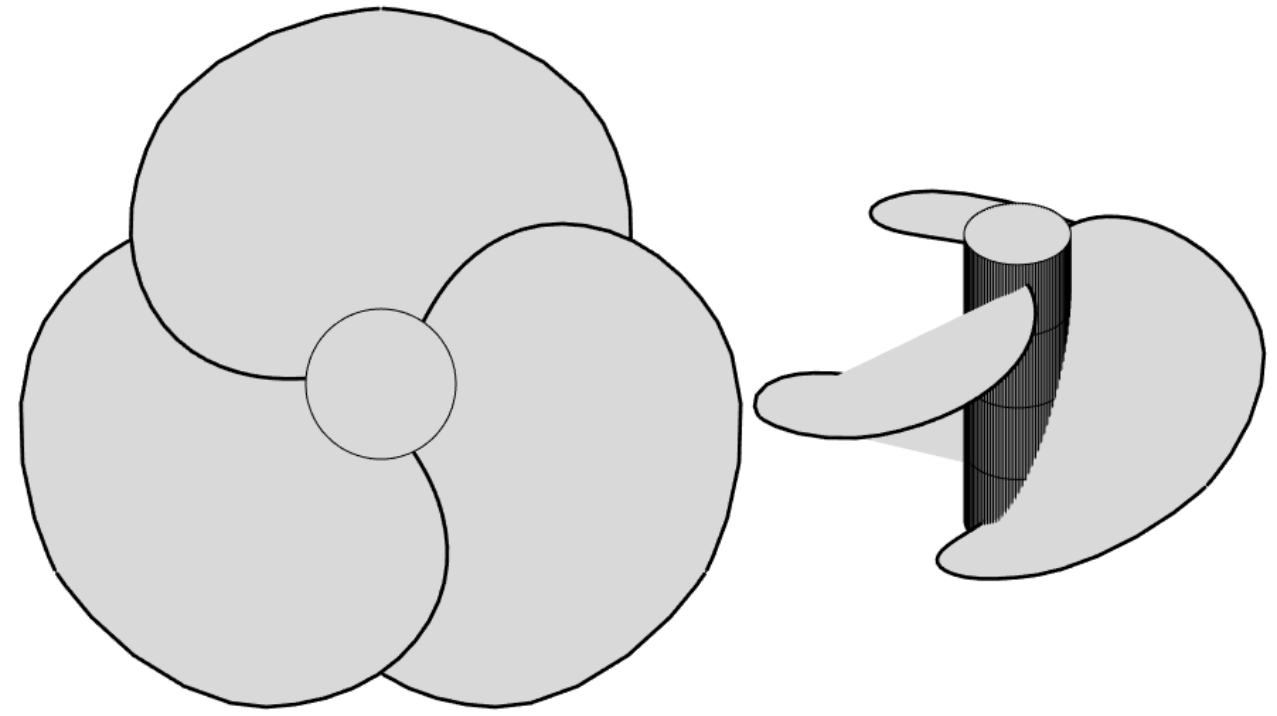

Source: Author

The source of experimental data is the same as for P4119 (DENNY, 1968). Therefore, the same experimental apparatus and associated measurements and uncertainties have been performed for this propeller. The numerical and experimental curves for $K_{T} \times J$ and $K_{Q} \times J$ are presented in figure 60. 
Table 4 - Propeller characteristics and blade outline. DTMB P4133.

\begin{tabular}{|c|c|c|c|c|c|c|}
\hline \multicolumn{7}{|c|}{$\begin{array}{l}\text { Number of blades, } \mathrm{Z}: 3 \\
\text { Hub diameter ratio: } 0.2 \\
\text { Expanded Area Ratio: } 1.212 \\
\text { Section mean line: NACA a }=0.8 \\
\text { Section thickness distribution: NACA } 66 \text { TMB mod } \\
\text { Design advance coefficient, J }=0.833\end{array}$} \\
\hline$\frac{r}{R}$ & $\frac{c}{D}$ & $\frac{P}{D}$ & $\frac{t_{0}}{c}$ & $\frac{f_{0}}{c}$ & $\theta_{S}$ & $\frac{z_{R}}{D}$ \\
\hline .20 & & . & & & & \\
\hline 20 & & & & & & \\
\hline 400 & 3096 & 976 & 0.0 & & & \\
\hline- & 784 & 75 & & & & \\
\hline 8 & 220 & & & & & \\
\hline & 244 & 073 & & & & \\
\hline 800 & 3694 & 73 & & & & \\
\hline .900 & 1220 & .073 & & & & \\
\hline 0.950 & & & & & & \\
\hline 1.000 & 0.0040 & 1.073 & 0.0038 & 0.0140 & 0.0000 & 0.0000 \\
\hline
\end{tabular}

Source: Author

For $K_{T} \times J$, comparing the influence of the source of 2-D data, the same trend as before is observed: the PLL-TFT values are higher than the other two, while those have similar values for practically the entire $J$ range. For $K_{Q} \times J$, more observable differences happen amongst the three numerical curves, although the PLL-Star-CCM+ and the PLL-Brockett curves are closer;

Comparing numerical and experimental values, the agreement for $K_{T}$ increases with $J$, evidencing the influence of the moderately/lightly loaded conditions on the formulation. Near the design coefficient, the agreement is yet not satisfactory, but better than in comparison to much lower $J$. For $K_{Q}$, the closer agreement happens from the Brockett-based PLL, and the agreement seems to be better for this coefficient.

Specially for $K_{T}$, the differences may be explained by the combination of both large EAR and blade loading condition; figure 61 shows $\frac{v_{a}}{V_{\infty}}$ and $\frac{v_{t}}{V_{t}}$ for $\frac{r}{R} \approx 0.7$ and $C_{T} \times J$ for P4133. For $J<0.8$, the moderately loading condition starts to loose, trend that can also be observed by $C_{T}$ at this $J\left(C_{T}>0.5\right)$. In classic PLL methods, a common practice is to use Lifting-Surface corrections for the angle of attack and camber; they might overlap or add on to the source of bidimensional data, but could bring the experimental and numerical curves close to each other. As these corrections have not been implemented on the current formulation, they might be a topic for future study. In terms of torque, the better agreement for $K_{Q}$ seems to be due to the better appropriateness of $C_{c}$ data for this propeller, as viscous effects are more prominent for larger blades. This trend indicates that 
Figure $60-K_{T}$ and $K_{Q}$ versus $J$. DTMB P4133.
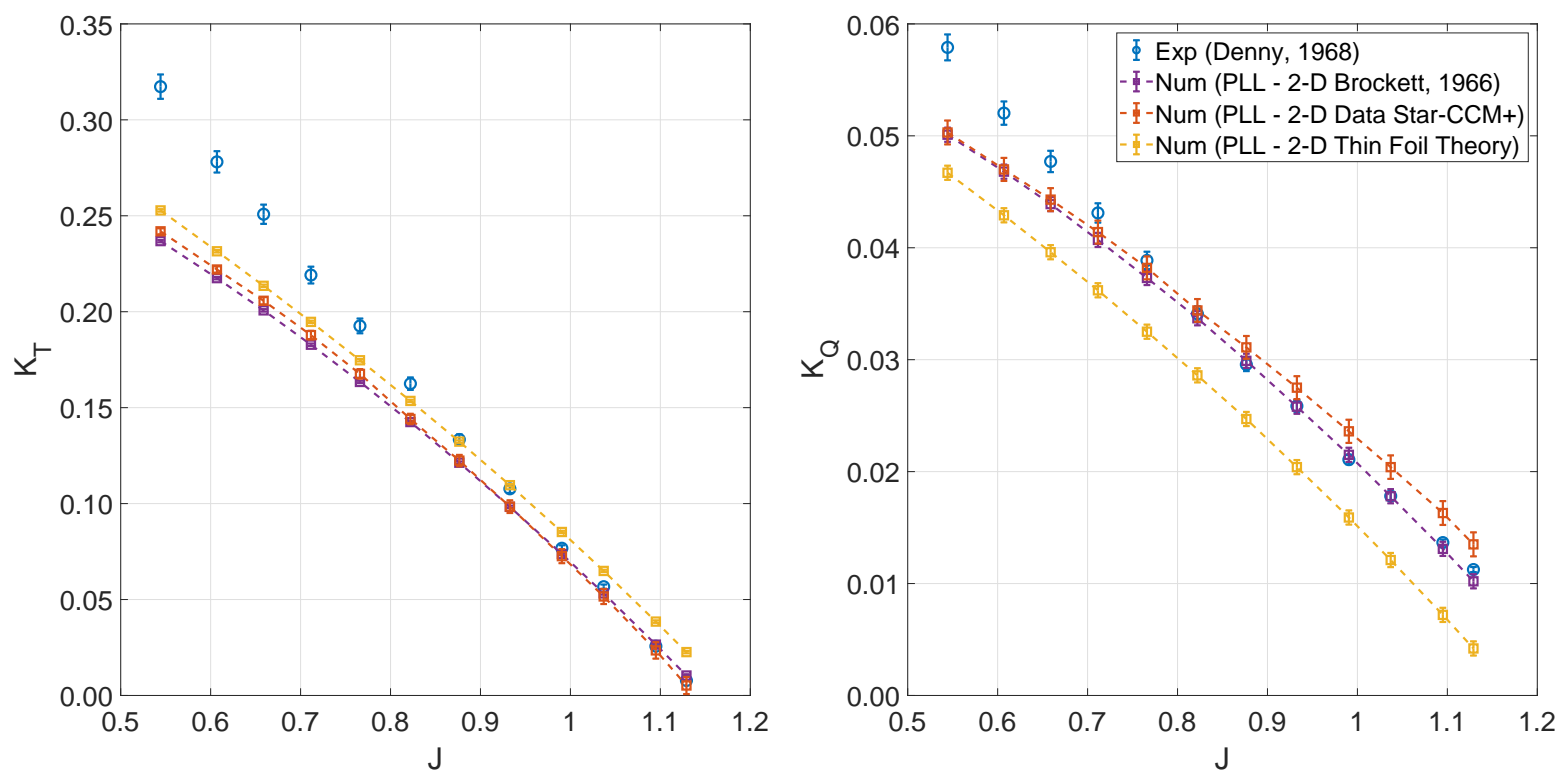

Source: Author

a unique value of $R e$ for simulating the blade sections might not be adequate.

Figure $61-\frac{v_{a}}{V_{\infty}} \times J$ and $\frac{v_{t}}{V_{t}} \times J$ for $\frac{r}{R}=0.7 C_{T} \times J$. DTMB P4133.
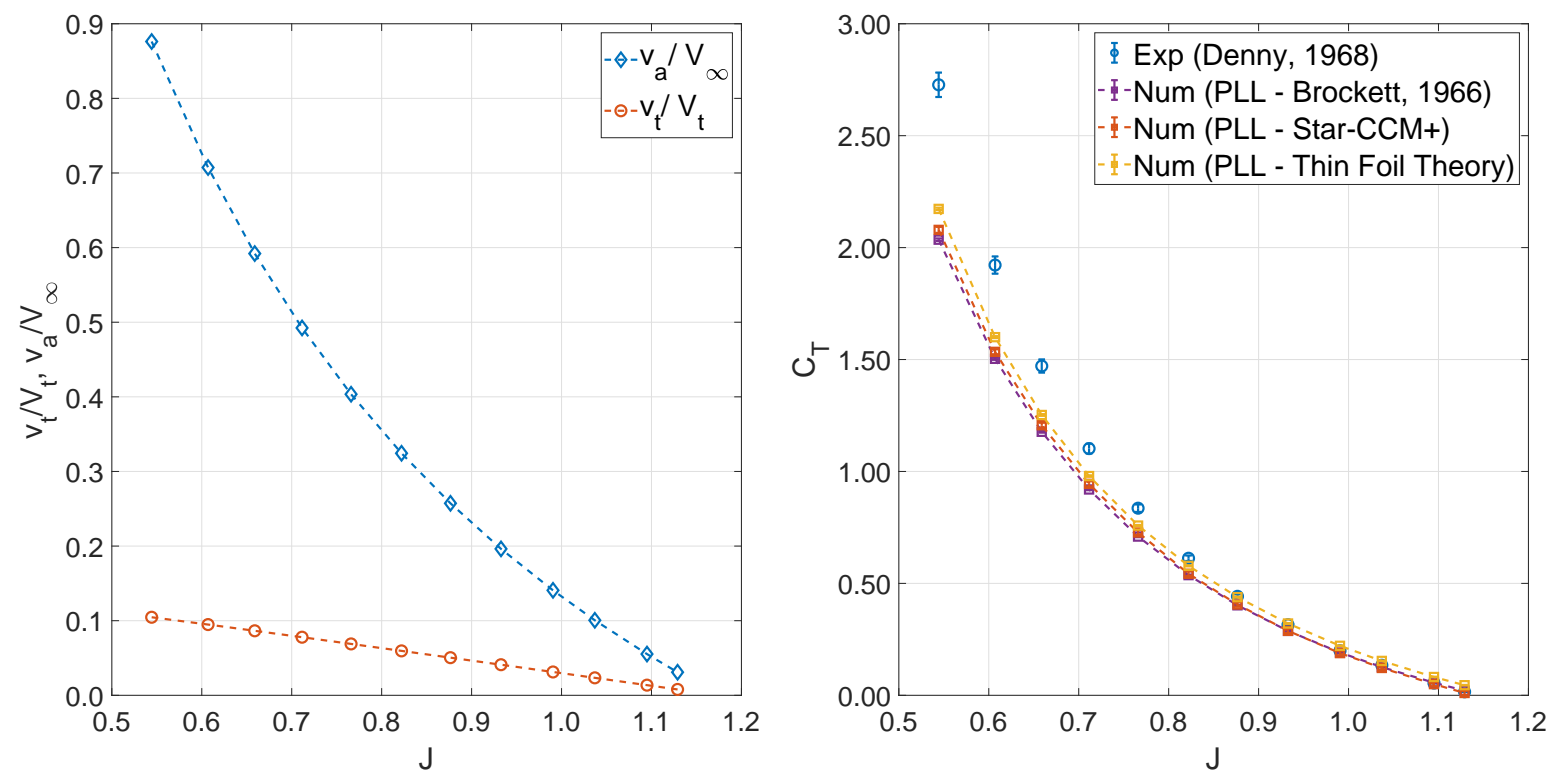

Source: Author

\subsubsection{DTMB 4381 Propeller}

DTMB P4381 is a five-bladed propeller with all characteristics but $\frac{c}{D}$ somewhat higher than P4119, but the important change is the increased number of blades and its 
effect on the PLL. The blade outline is described in table 5, while a propeller drawing is presented in figure 62 .

Figure 62 - Propeller drawing. DTMB P4381.

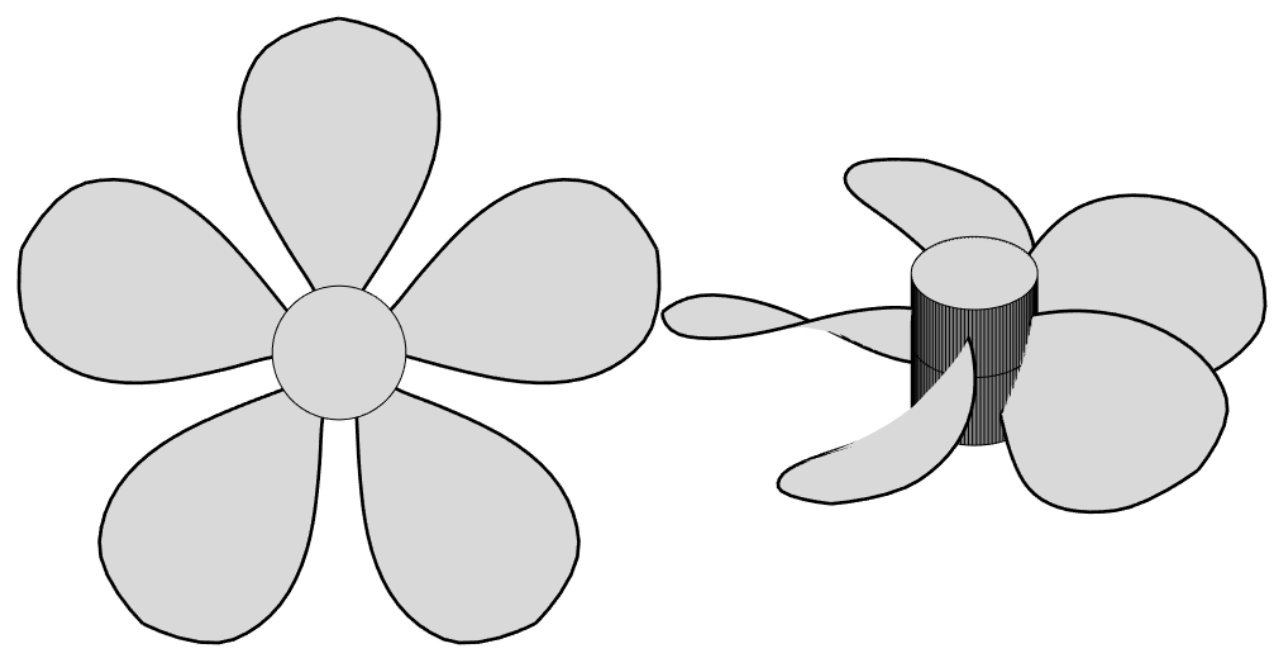

Source: Author

Table 5 - Propeller characteristics and blade outline. DTMB P4381.

Number of blades, Z: 5

Hub diameter ratio: 0.2

Expanded Area Ratio: 0.725

Section mean line: NACA a $=0.8$

Section thickness distribution: NACA66 TMB mod

Design advance coefficient, $\mathrm{J}=0.889$

\begin{tabular}{ccccccc}
\hline$\frac{r}{R}$ & $\frac{c}{D}$ & $\frac{P}{D}$ & $\frac{t_{0}}{c}$ & $\frac{f_{0}}{c}$ & $\theta_{S}$ & $\frac{z_{R}}{D}$ \\
\hline 0.200 & 0.1740 & 1.332 & 0.2471 & 0.0350 & 0.0000 & 0.0000 \\
0.250 & 0.2020 & 1.338 & 0.1931 & 0.0360 & 0.0000 & 0.0000 \\
0.300 & 0.2290 & 1.345 & 0.1528 & 0.0360 & 0.0000 & 0.0000 \\
0.400 & 0.2750 & 1.358 & 0.1055 & 0.0340 & 0.0000 & 0.0000 \\
0.500 & 0.3120 & 1.336 & 0.0769 & 0.0300 & 0.0000 & 0.0000 \\
0.600 & 0.3370 & 1.280 & 0.0564 & 0.0240 & 0.0000 & 0.0000 \\
0.700 & 0.3470 & 1.210 & 0.0403 & 0.0190 & 0.0000 & 0.0000 \\
0.800 & 0.3340 & 1.137 & 0.0299 & 0.0140 & 0.0000 & 0.0000 \\
0.900 & 0.2800 & 1.066 & 0.0214 & 0.0120 & 0.0000 & 0.0000 \\
0.950 & 0.2100 & 1.031 & 0.0190 & 0.0070 & 0.0000 & 0.0000 \\
1.000 & 0.0000 & 0.995 & 0.0000 & 0.0000 & 0.0000 & 0.0000 \\
\hline
\end{tabular}

Source: Author

Experimental data come from open-water propulsion tests of four model propellers, named P438X, that were conducted in the Naval Ship Research and Development Center (NSRDC) deep-water basin (BOSWELL, 1971); the apparatus was instrumented with a 
gravity dynamometer and the tests were run at $468 R P M$ and at an advance velocities that permitted a variation in $J$ from 0.37 to 1.07, approximately. Nevertheless, no information was provided about either the input or experimental uncertainties.

Figure 63 shows the agreement between numerical and experimental curves; in terms of $K_{T}$, this time PLL-TFT and PLL-Star-CCM+ agree better, but the PLL-Brockett seems to agree better with experimental data for most of the $J$ range. In terms of $K_{Q}$, the PLL-Star-CCM+ overestimates the coefficient for most of $J$, while the other two curves, in addition to agreeing better to each other, agree better with the experimental data as well.

Comparison with experimental data shows that, for $K_{T}$, the same trend is observed as on the previous cases: near design $J$, the agreement is quite satisfactory, while as $J$ decreases much, the agreement starts to lose. Again the trend is likely related to not satisfying the moderately loaded assumption at these advance ratios, as can be observed by figure 64: for $J<0.8$ the assumption already starts to loose as indicated by the axial induced velocity, although the numerical values for $K_{T}$ and $C_{T}$ near this advance coefficient are relatively close to the experimental. Note, however, that near $J=0.8, C_{T}$ is already near unity. For $K_{Q}$, the inclusion of $C_{c}$ data through Star-CCM+ does not seem to contribute to its calculation, as they overestimate $K_{Q}$ and the results from either Brockett's expressions or the TFT agree better with experimental data, so this result is analogous to the P4119 case.

Figure $63-K_{T}$ and $K_{Q}$ versus $J$. DTMB P4381.
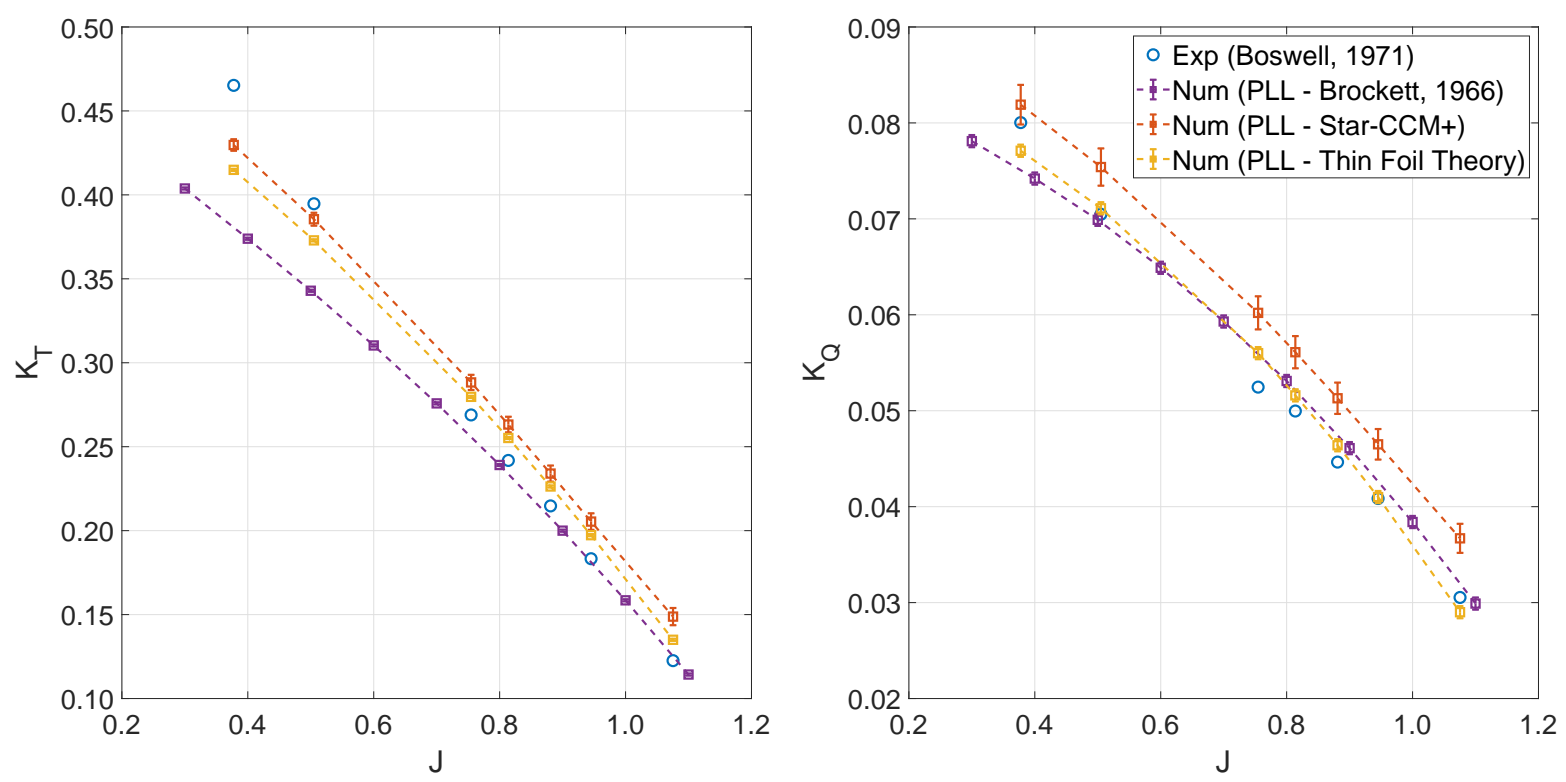

Source: Author 
Figure $64-\frac{v_{a}}{V_{\infty}} \times J$ and $\frac{v_{t}}{V_{t}} \times J$ for $\frac{r}{R}=0.7$ and $C_{T}$ versus $J$. DTMB P4381.
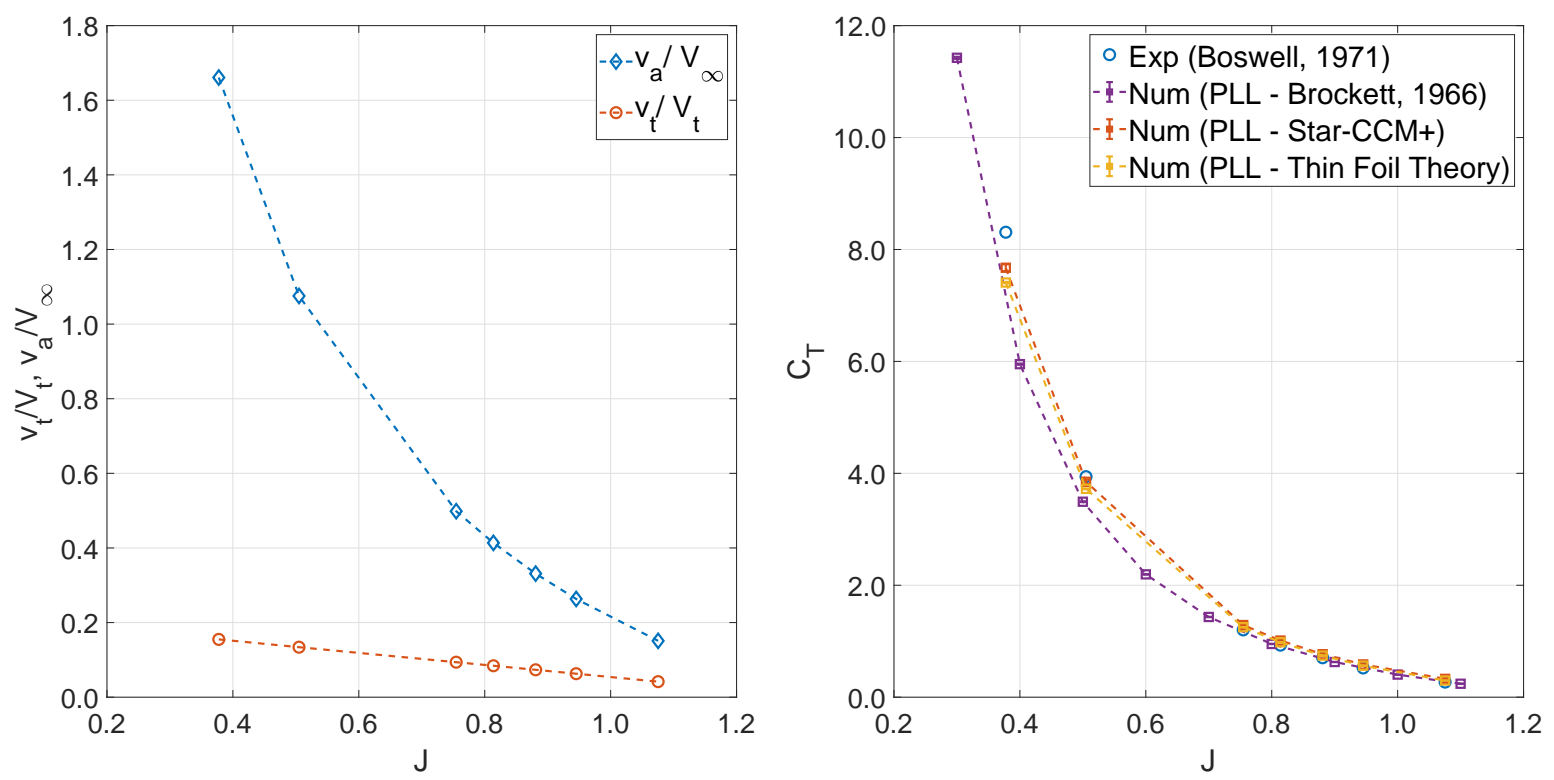

Source: Author

\subsubsection{H1 Propeller}

The $\mathrm{H} 1$ is a propeller from the Kings College Admiralty (KCA) (also known as Gawn-Burrill) family. When developed, this series comprised a SS of 30 three-bladed, $406 \mathrm{~mm}$ models having a range of pitch ratios and EAR; all KCA propellers had uniform face pitch and ogival foil sections - i.e., a combination of parabolic mean lines with parabolic thicknesses (KERWIN; HADLER, 2010) - with subtle lifts at both leading and trailing edges, elliptical blade outlines and boss-to-propeller diameter ratios of 0.2. The H1 propeller, which following the same characteristics, is depicted in figure 65, and its outline is described in table 6 . This propeller of interest for the validation as its outline is more or less similar to P4119, but with a different blade section family and with expressions for $K_{T}$ and $K_{Q}$ derived from the SS approach.

The Institute for Technological Research (IPT) Cavitation Tunnel was the place for experimental analysis of the $\mathrm{H} 1$ propeller, whose measurements of thrust and torque were carried through two pendulum systems that were connected to the drive motor and the load cells. This configuration allows a range of measurements on thrust from 20 to $1000 \mathrm{~N}$, with an associated error of $0.05 \%$. The modularity of the mechanism permits both low and high loads tests solely by changing the cells. The velocity at the test section is calculated employing transistors that measure the pressure difference between the Venturi-like inlet and outlet sections. Thus, the average flow velocity is calculated by Bernoulli's and continuity equations. Although the results of the hydrodynamic analysis are still under meticulous analysis, part of them has already been published (KATSUNO; DANTAS, 2017). 
Figure 65 - Propeller drawing. H1.

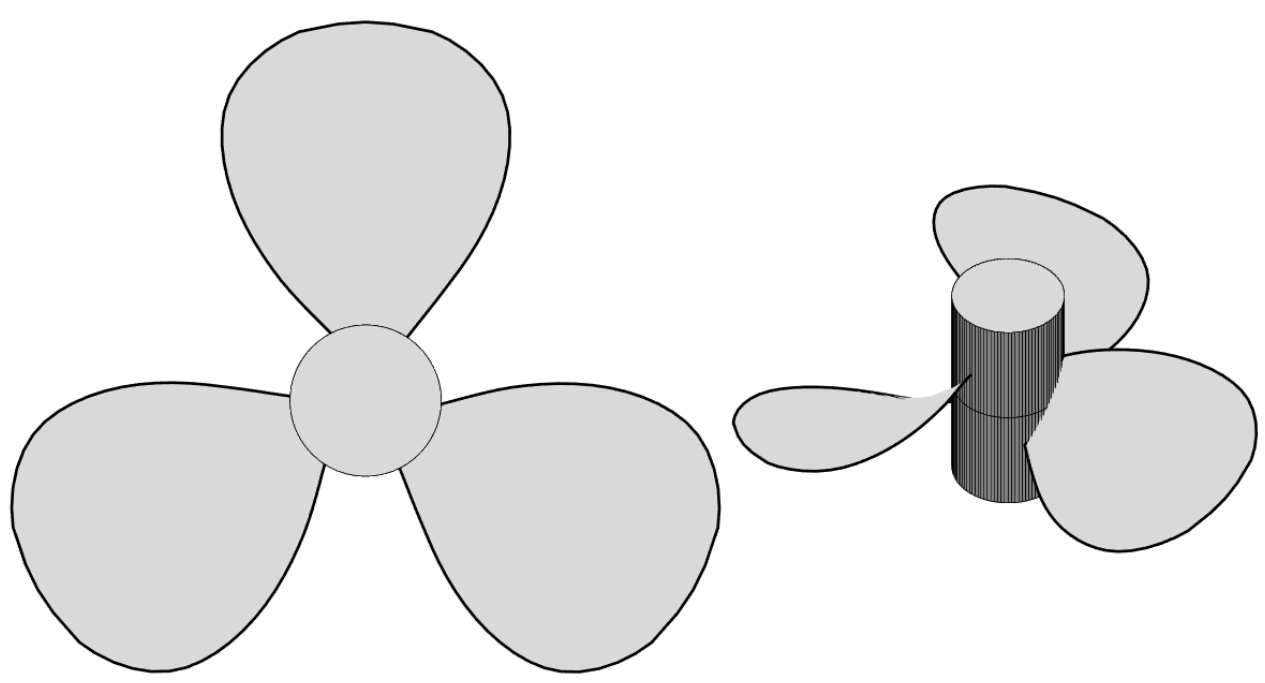

Source: Author

Table 6 - Propeller characteristics and blade outline. H1.

Number of blades, Z: 3

Hub diameter ratio: 0.2

Expanded Area Ratio: 0.500

Section mean line: parabolic

Section thickness distribution: parabolic

Design advance coefficient, $\mathrm{J}=0.700$

\begin{tabular}{ccccccc}
\hline$\frac{r}{R}$ & $\frac{c}{D}$ & $\frac{P}{D}$ & $\frac{t_{0}}{c}$ & $\frac{f_{0}}{c}$ & $\theta_{S}$ & $\frac{z_{R}}{D}$ \\
\hline 0.200 & 0.1800 & 0.865 & 0.1627 & 0.0385 & 0.0000 & 0.0000 \\
0.250 & 0.2250 & 0.865 & 0.1627 & 0.0338 & 0.0000 & 0.0000 \\
0.375 & 0.2617 & 0.865 & 0.1007 & 0.0270 & 0.0000 & 0.0000 \\
0.500 & 0.3250 & 0.865 & 0.0627 & 0.0176 & 0.0000 & 0.0000 \\
0.625 & 0.3767 & 0.865 & 0.0415 & 0.0116 & 0.0000 & 0.0000 \\
0.750 & 0.4167 & 0.865 & 0.0278 & 0.0073 & 0.0000 & 0.0000 \\
0.875 & 0.4283 & 0.865 & 0.0171 & 0.0039 & 0.0000 & 0.0000 \\
0.938 & 0.4262 & 0.865 & 0.0126 & 0.0024 & 0.0000 & 0.0000 \\
1.000 & 0.4167 & 0.865 & 0.0126 & 0.0000 & 0.0000 & 0.0000 \\
\hline
\end{tabular}

Source: Author

Figure 66 presents the comparison among experimental, numerical an the SS approach results. This time, however, there is no experimental expressions from Brockett (or similar) to be used in the calculations of $C_{n}$ and $C_{c}$. In terms of $K_{T}$, the difference between using Star-CCM+ or the TFT is not significant, but the PLL-Star-CCM+ results are closer to the experimental data. For $K_{Q}$, the inclusion of viscous data enhances the coefficient estimates, and the results are reasonably near the SS and experimental values, for $J$ near design and higher. Comparison with experimental data shows that also near 
design $J$ and higher numerical and experimental $K_{T}$ agree satisfactorily; even for lower $J$ (and near design), the agreement is satisfactory. The moderately loaded condition is corroborated by the magnitude of the induced velocities, as shown on figure 67 : for $J \geq 0.7$, both $\frac{v_{a}}{V_{\infty}} \times J$ and $\frac{v_{t}}{V_{t}} \times J$ are lower than 0.2 . Additionally, $C_{T} \approx 0.4$, while for $J \approx 0.62$, $C_{T} \approx 0.8$; thus, far from these values as the assumption is lost, so the agreement.

Figure $66-K_{T}$ and $K_{Q}$ versus $J . \mathrm{H} 1$.
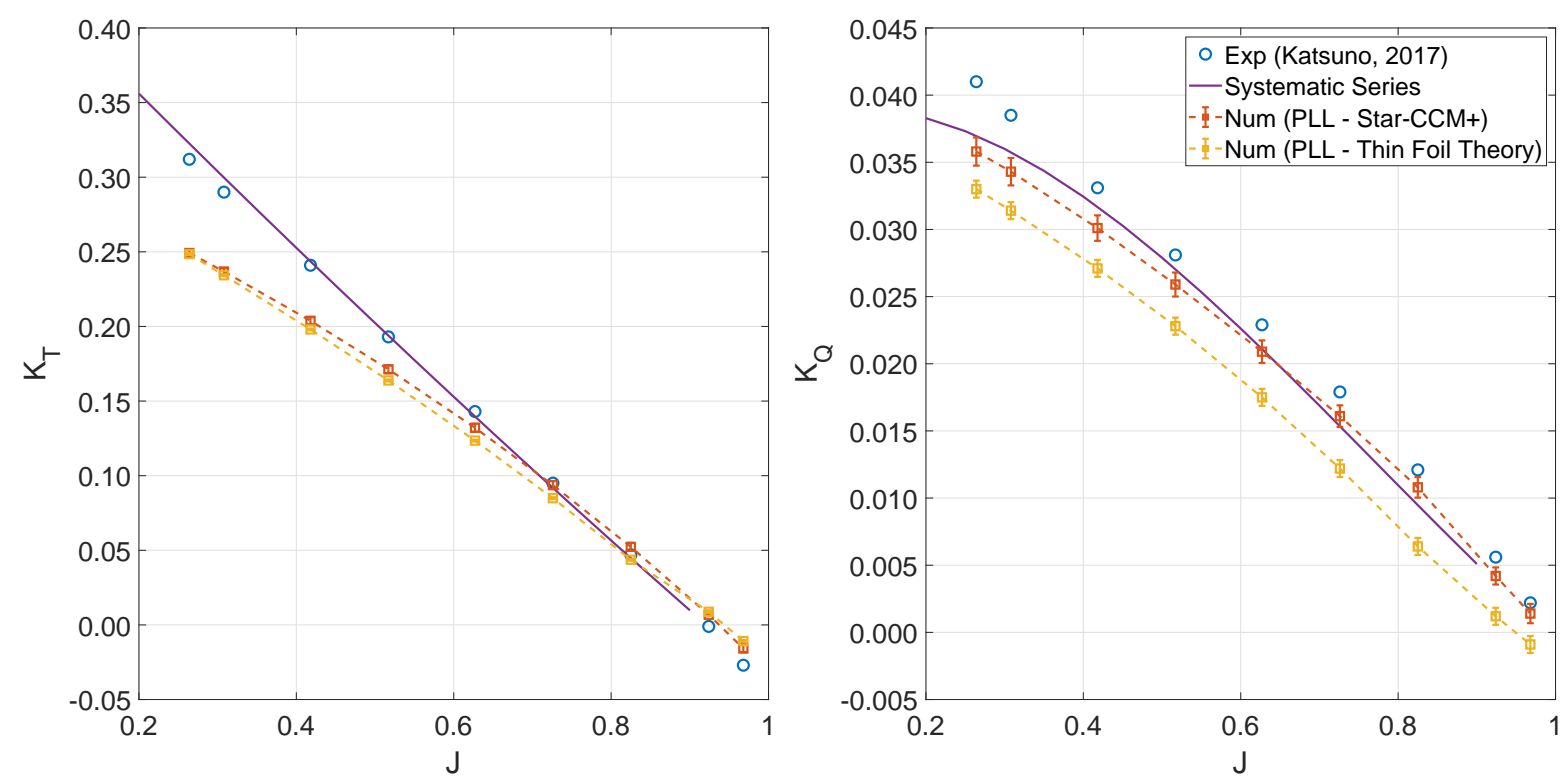

Source: Author

Figure $67-\frac{v_{a}}{V_{\infty}} \times J$ and $\frac{v_{t}}{V_{t}} \times J$ for $\frac{r}{R}=0.7$ and $C_{T}$ versus $J$. H1.
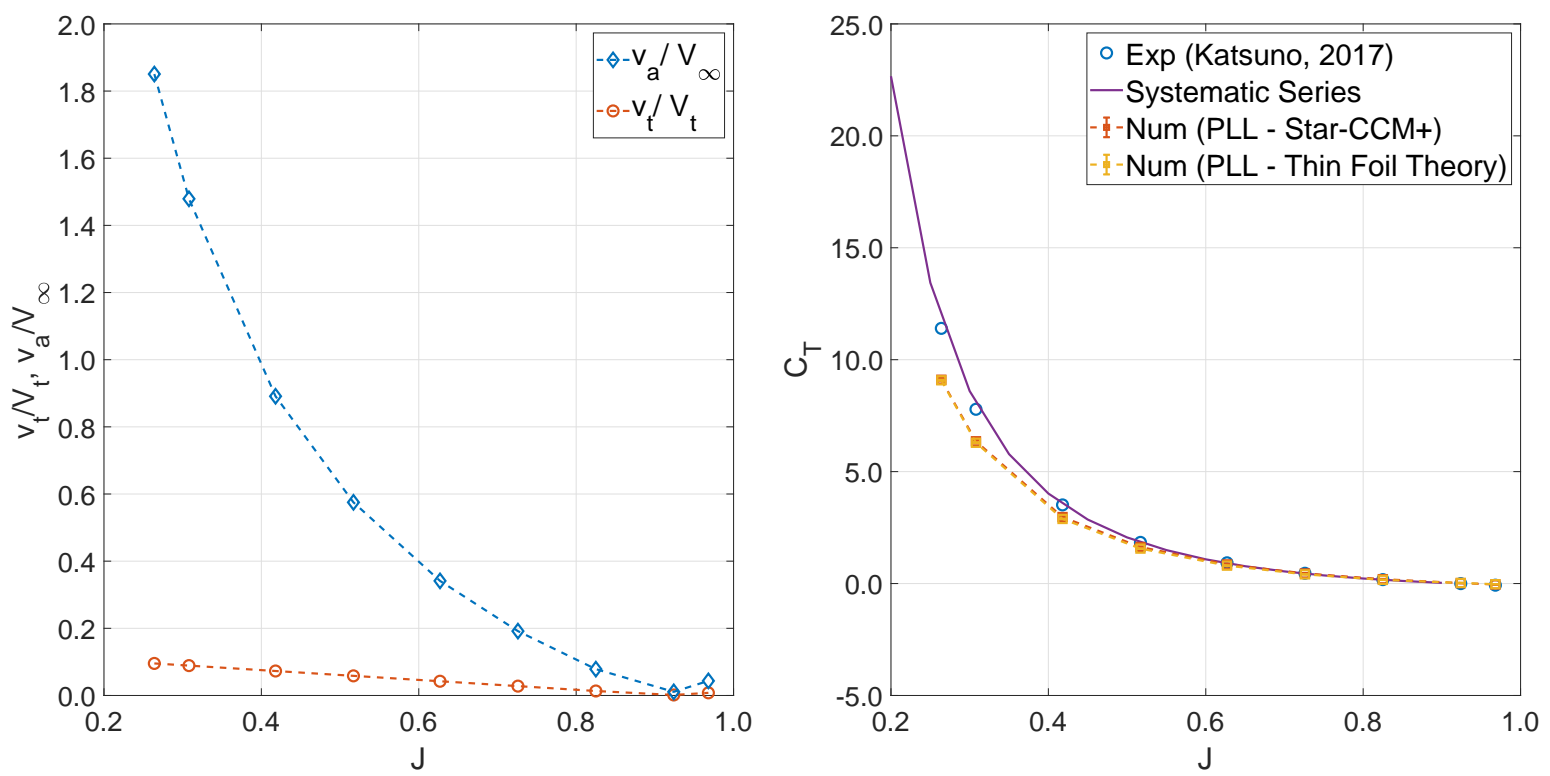

Source: Author 


\subsubsection{MOD5 Propeller}

MOD5 is a seven-bladed propeller with a quadratic skew distribution that has been conceived to be used on the Defense Advance Research Projects Agency (DARPA) SUBOFF model (GROVES; HUANG; CHANG, 1989), to operate in between moderately and lightly loaded conditions near operation design. Several numerical and experimental studies have been carried out at IPT to characterize the propeller (ESTEVES et al., 2018; Silva Jr et al., 2019). The blade outline is presented on table 7 , while a drawing of the propeller is presented in figure 68.

Figure 68 - Propeller drawing. MOD5.

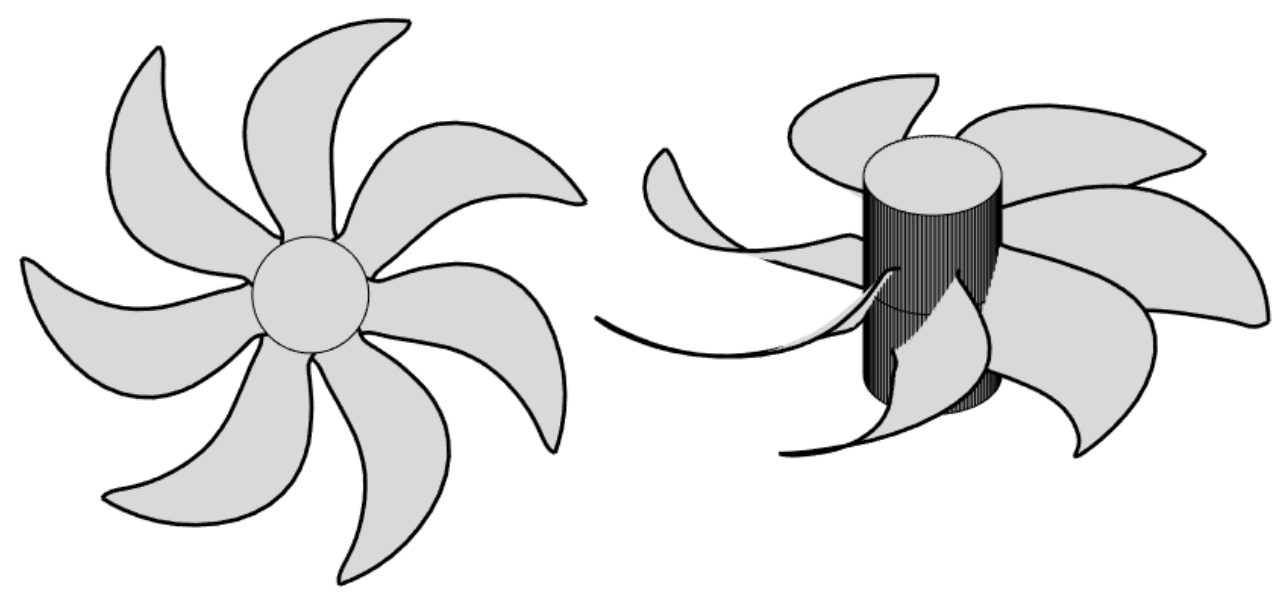

Source: Author

For the MOD5, experiments have also been conducted at the IPT Cavitation Tunnel, in a relatively large range of advance ratios (from 0.2 to 1.2, approximately). As in the case of $\mathrm{H} 1$, the necessary corrections due to the measurement uncertainty and blockage effects of the tunnel have been considered, and although part of the results have been published (KATSUNO; DANTAS, 2017), a more throughout analysis must be carried to correctly quantify the associated uncertainties in thrust and torque (ESTEVES et al., 2018; Silva Jr et al., 2019).

Figure 69 presents the comparison between experimental and numerical $K_{T} \times J$ and $K_{Q} \times J$ curves. The use of either Brockett expressions or the results from Star-CCM+ does not change significantly the results obtained, in terms of $K_{T}$, while the results for the PLL-TFT are overestimated. For $K_{Q}$, the three numerical results slightly depart from one another and they all underestimate the experimental $K_{Q}$. Note that, differently than the other propellers, all curves have a remarkably satisfactory agreement with experimental data despite the advance coefficient, corroborating the appropriateness of the PLL to simulate geometries that have some degree of skew.

It is worth noting that $\frac{v_{a}}{V_{\infty}}$ is relatively high for $J<0.7$ - figure 70 , but still the 
Table 7 - Propeller characteristics and blade outline. MOD5.

\begin{tabular}{|c|c|c|c|c|c|c|}
\hline $\begin{array}{l}\text { Numb } \\
\text { Hub } \\
\text { Expar } \\
\text { Sectio } \\
\text { Sectio } \\
\text { Desig }\end{array}$ & $\begin{array}{l}\text { r of bla } \\
\text { ameter } \\
\text { led Are } \\
\text { mean } 1 \\
\text { thickn } \\
\text { advanc }\end{array}$ & $\begin{array}{l}\text { les, Z: } \\
\text { atio: } 0 \\
\text { Ratio } \\
\text { ne: NA } \\
\text { ss distr } \\
\text { coeffic }\end{array}$ & $\begin{array}{l}0.604 \\
\text { CA a }= \\
\text { ibution: } \\
\text { ent, J = }\end{array}$ & $\begin{array}{l}.8 \\
\text { NACA66 } \\
0.919\end{array}$ & TMR m & \\
\hline$\frac{r}{R}$ & $\frac{c}{D}$ & $\frac{P}{D}$ & $\frac{t_{0}}{c}$ & $\frac{f_{0}}{c}$ & $\theta_{S}$ & $\frac{z_{R}}{D}$ \\
\hline 0.200 & 0.1812 & 0.900 & 0.1718 & 0.0000 & 0.0000 & 0.0000 \\
\hline 0.250 & 0.1814 & 1.053 & 0.1599 & 0.0501 & -2.7300 & 0.0000 \\
\hline 0.300 & 0.1830 & 1.092 & 0.1449 & 0.0426 & -4.9400 & 0.0000 \\
\hline 0.400 & 0.1856 & 1.147 & 0.1213 & 0.0305 & -7.7500 & 0.0000 \\
\hline 0.500 & 0.1901 & 1.197 & 0.1036 & 0.0235 & -8.4400 & 0.0000 \\
\hline 0.600 & 0.1945 & 1.214 & 0.0933 & 0.0193 & -7.0000 & 0.0000 \\
\hline 0.700 & 0.1936 & 1.216 & 0.0919 & 0.0174 & -3.4400 & 0.0000 \\
\hline 0.800 & 0.1741 & 1.204 & 0.0905 & 0.0187 & 2.2500 & 0.0000 \\
\hline 0.900 & 0.1226 & 1.145 & 0.0891 & 0.0242 & 10.0600 & 0.0000 \\
\hline 0.950 & 0.0821 & 1.098 & 0.0884 & 0.0335 & 14.7700 & 0.0000 \\
\hline 1.000 & 0.0000 & 1.044 & 0.0884 & 0.0000 & 20.0000 & 0.0000 \\
\hline
\end{tabular}

Source: Author

Figure $69-K_{T}$ and $K_{Q}$ versus $J$. MOD5.
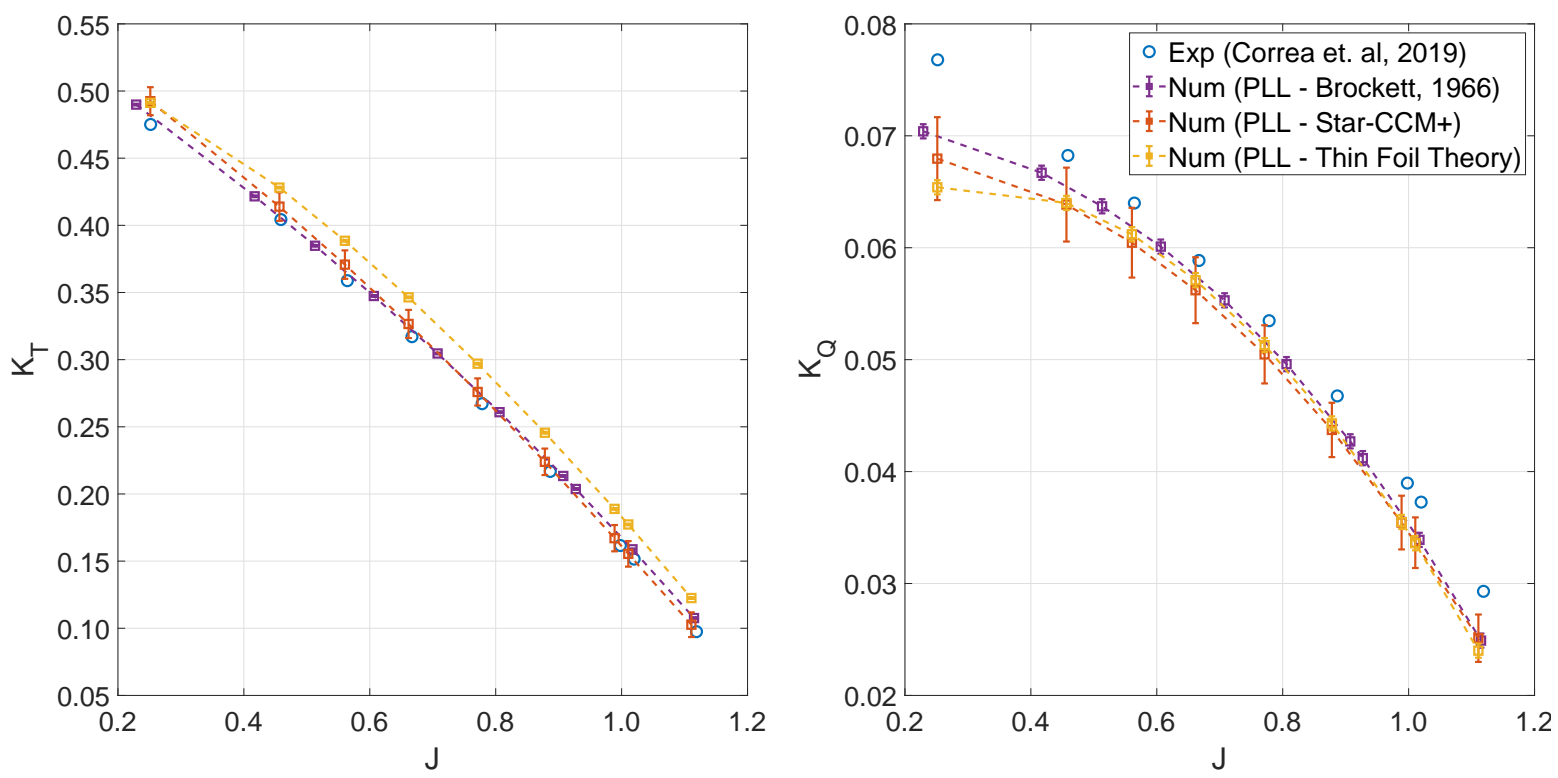

Source: Author

agreement between numerical and experimental data is satisfactory. In terms of $C_{T}$, this agreement is also observed for most of the $J$ range. This trend is not usual for PLL methods and also is counter-intuitive, as the moderately loaded assumption would have been lost. 
Therefore, further investigation must be conducted to understand such an agreement, but it might be associated with the high blade AR.

Figure $70-\frac{v_{a}}{V_{\infty}} \times J$ and $\frac{v_{t}}{V_{t}} \times J$ for $\frac{r}{R}=0.7$ and $C_{T}$ versus $J$. MOD5.
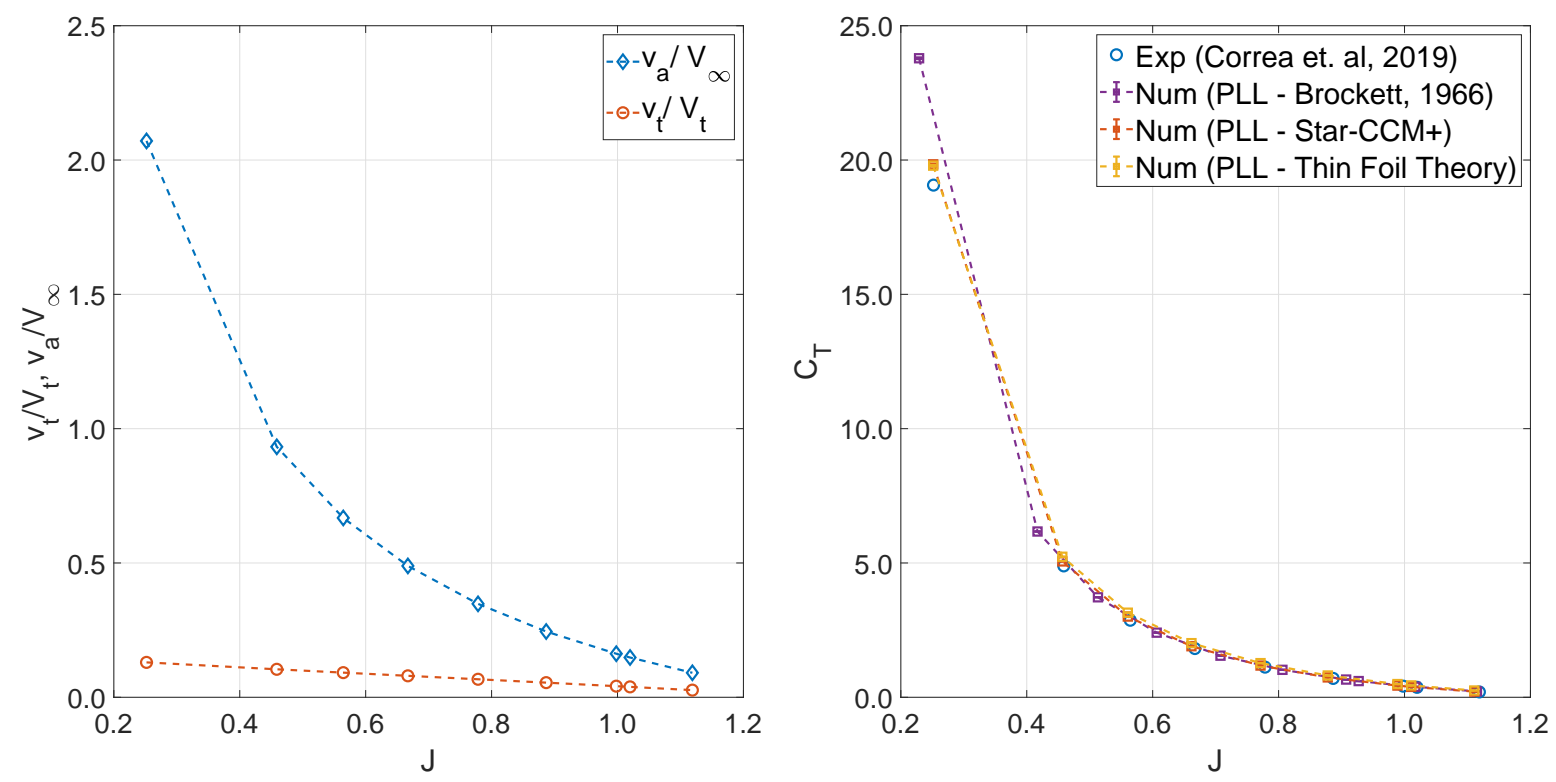

Source: Author

\subsubsection{PPTC Propeller}

Intending to provide researchers a standard test propeller case within the SchiffbauVersuchsanstalt Potsdam Model Basin (SVA), the model basin developed and investigated the open-water and cavitation characteristics of the controllable pitch propeller VP1304 also known as Potsdam Propeller Test Case (PPTC) (BARKMANN, 2011). The test case offers researchers the possibility to test and validate their numerical tools, thus contributing to the enhancement of numerical predictions. The PPTC blade outline is presented on table 8, while its drawing is presented in figure 71.

Figure 72 presents $K_{T} \times J$ and $K_{Q} \times J$ for this propeller, which has both skew and rake distributions. The use of $2-\mathrm{D}$ data from Star-CCM+ significantly improves the estimates for $K_{T}$, which starts agreeing satisfactorily to the experimental data near the design advance ratio, as in the previous cases. In terms of $K_{Q}$, the results from either PLL are essentially the same, and they agree with experimental data at approximately the design $J$ as well; as in some previous cases, the use of 2-D data does not seem to impact the results, meaning that the effects of viscosity might not be as important as the ones from induced drag.

Figure 73 illustrates how this propeller is extremely loaded at low $J(J<0.8)$, thus being in a region that cannot be accurately predicted by the current formulation. In fact, it is only near $J \geq 1.0$ that $\frac{v_{a}}{V_{\infty}} \times J$ and $\frac{v_{t}}{V_{t}} \times J$ ratios are near the typical values for 
Figure 71 - Propeller drawing. PPTC.

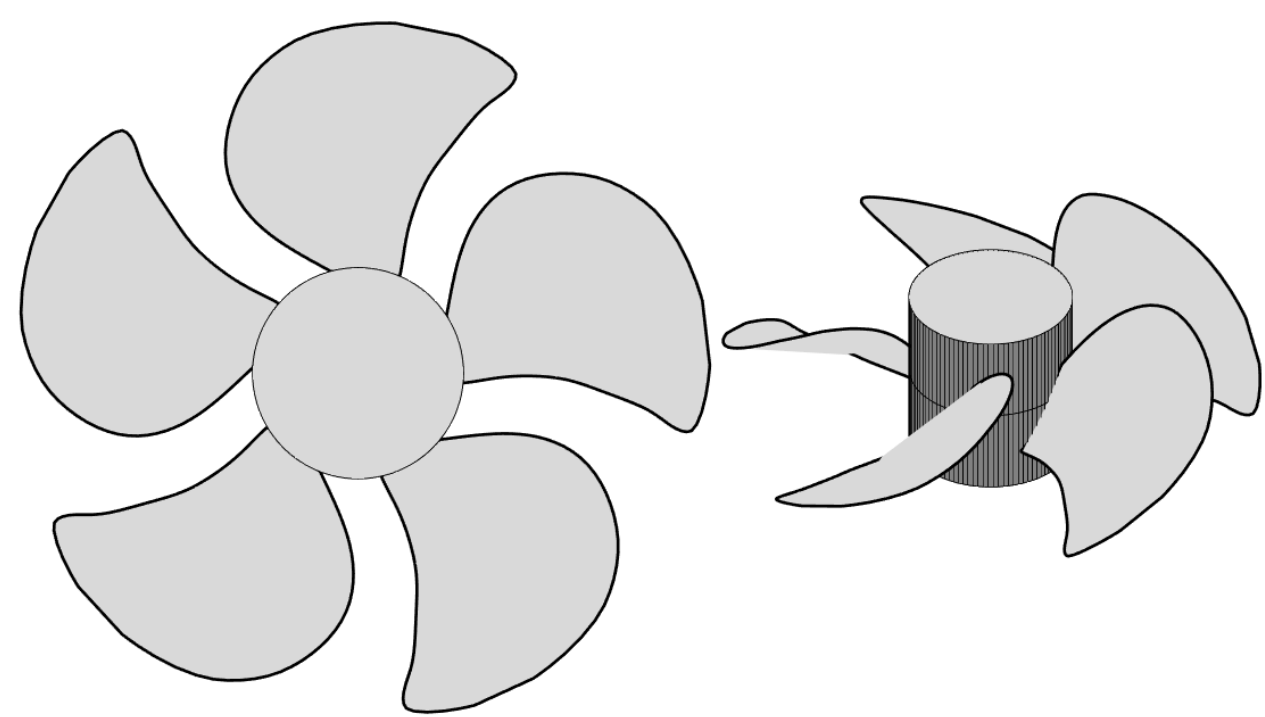

Source: Author

Table 8 - Propeller characteristics and blade outline. PPTC.

Number of blades, Z: 5

Hub diameter ratio: 0.3

Expanded Area Ratio: 0.779

Section mean line: PPTC

Section thickness distribution: PPTC

Design advance coefficient, $\mathrm{J}=1.2830$

\begin{tabular}{ccccccc}
\hline$\frac{r}{R}$ & $\frac{c}{D}$ & $\frac{P}{D}$ & $\frac{t_{0}}{c}$ & $\frac{f_{0}}{c}$ & $\frac{x_{S}}{D}$ & $\frac{z_{R}}{D}$ \\
\hline 0.300 & 0.1800 & 1.400 & 0.3006 & 0.0150 & 0.0000 & 0.0000 \\
0.350 & 0.2250 & 1.455 & 0.2142 & 0.0200 & 0.0020 & 0.5000 \\
0.400 & 0.2617 & 1.505 & 0.1634 & 0.0240 & 0.0050 & 1.2500 \\
0.500 & 0.3250 & 1.578 & 0.1002 & 0.0290 & 0.0134 & 3.3500 \\
0.600 & 0.3767 & 1.620 & 0.0642 & 0.0301 & 0.0235 & 5.8750 \\
0.700 & 0.4167 & 1.635 & 0.0425 & 0.0297 & 0.0300 & 7.5000 \\
0.800 & 0.4283 & 1.614 & 0.0304 & 0.0285 & 0.0295 & 7.3750 \\
0.850 & 0.4262 & 1.582 & 0.0260 & 0.0279 & 0.0265 & 6.6250 \\
0.900 & 0.4167 & 1.531 & 0.0228 & 0.0256 & 0.0218 & 5.4500 \\
0.950 & 0.3767 & 1.458 & 0.0214 & 0.0233 & 0.0161 & 4.0333 \\
0.975 & 0.3147 & 1.411 & 0.0202 & 0.0218 & 0.0132 & 3.3000 \\
1.000 & 0.1000 & 1.355 & 0.0188 & 0.0020 & 0.0100 & 2.5000 \\
\hline
\end{tabular}

Source: Author

the moderately and lightly loaded assumptions to be valid. This explains the agreement between numerical and experimental coefficients that only happens to start from this region. The highly loading condition can also be observed by the $C_{T}$ trend: for $J \approx 0.2$, $C_{T}$ is near 100 , and still reasonably higher than 1 for $J \approx 0.5$. The agreement under 
Figure $72-K_{T}$ and $K_{Q}$ versus $J$ PPTC.
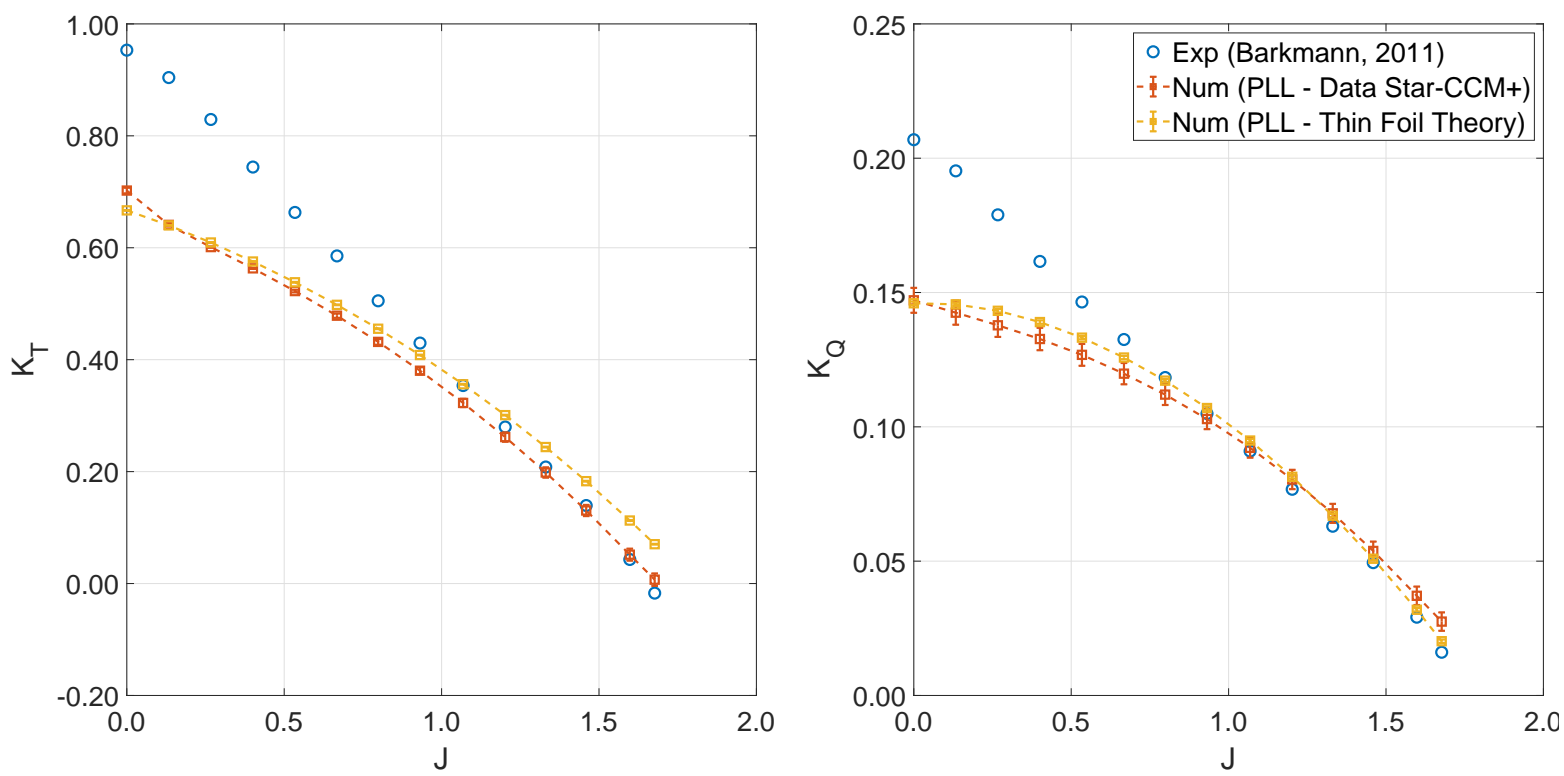

Source: Author

the moderately loaded condition implies that the present formulation seems suitable for simulating skewed and raked propellers as well, at least to some extent.

Figure $73-\frac{v_{a}}{V_{\infty}} \times J$ and $\frac{v_{t}}{V_{t}} \times J$ for $\frac{r}{R}=0.7 C_{T} \times K_{T}$. PPTC.
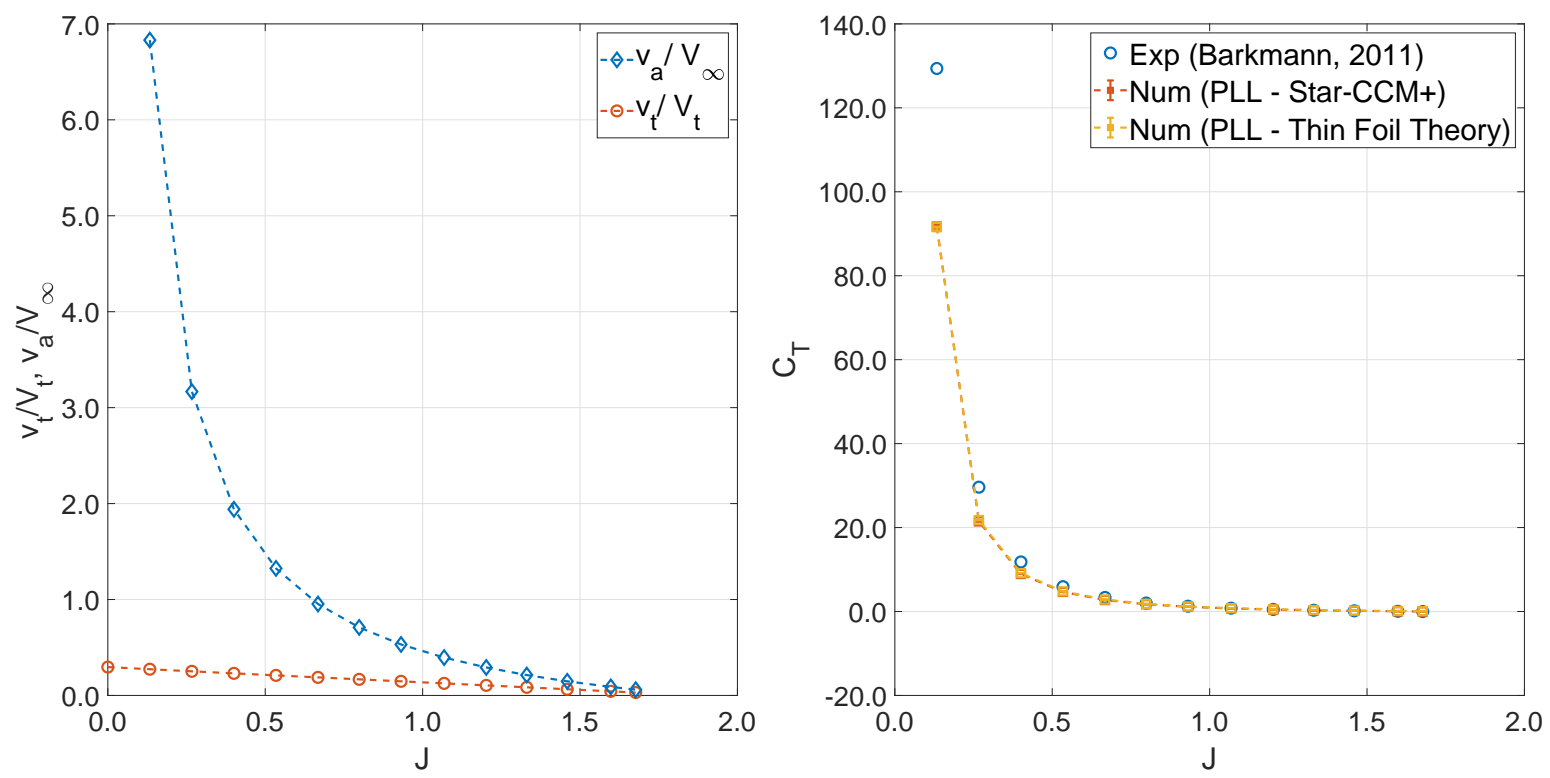

Source: Author

\subsubsection{Validation Tables and Conclusions about Validation}

A formal validation, in form of tables containing numerical (Star-CCM+ as source of 2-D data) and experimental values along with the respective uncertainties (when- 
ever available) is presented for the propeller cases discussed. Three values of the advance ratio were used in an attempt to distinguish - whenever feasible - among lightly $\left(C_{T} \leq 0.25, \frac{u_{t}}{V_{T}} \& \frac{u_{a}}{V_{\infty}} \leq 0.2\right)$, moderately $\left(0.2<C_{T} \leq 0.70, \frac{u_{t}}{V_{T}} \& \frac{u_{a}}{V_{\infty}} \leq 0.2\right)$, and heavily $\left(\frac{u_{t}}{V_{T}} \& \frac{u_{a}}{V_{\infty}}>0.2\right)$ loading conditions; the value of $J$ nearest design $J_{D}$ was also validated for each propeller. Tables 9 and 10 summarize these values.

Table 9 - Validation for the Propeller Lifting-Line formulation. Thrust Coefficient

\begin{tabular}{|c|c|c|c|c|c|c|c|c|}
\hline & Propeller & & P4119 & P4133 & P4381 & H1 & MOD5 & PPTC \\
\hline \multirow{6}{*}{$J_{L}$} & \multirow{2}{*}{ Experimental } & $K_{T}$ & 0.034 & 0.02567 & 0.1226 & -0.001 & 0.1515 & 0.1394 \\
\hline & & $u_{K_{T}}$ & 0.001 & 0.00051 & $\mathrm{~N} / \mathrm{A}$ & $\mathrm{N} / \mathrm{A}$ & $\mathrm{N} / \mathrm{A}$ & $\mathrm{N} / \mathrm{A}$ \\
\hline & \multirow{4}{*}{ Numerical } & $K_{T}$ & 0.031 & 0.02350 & 0.1488 & 0.007 & 0.1554 & 0.1305 \\
\hline & & $u_{K_{T}}$ & 0.005 & 0.00430 & 0.0051 & 0.001 & 0.0095 & 0.0100 \\
\hline & & $\mathrm{E}$ & -0.003 & -0.00217 & 0.0262 & 0.008 & 0.0039 & -0.0089 \\
\hline & & $u_{v}$ & 0.005 & 0.00433 & 0.0051 & 0.001 & 0.0095 & 0.0100 \\
\hline \multirow{6}{*}{$J_{M}$} & \multirow{2}{*}{ Experimental } & $K_{T}$ & 0.120 & 0.10770 & 0.1833 & 0.047 & 0.2168 & 0.2797 \\
\hline & & $u_{K_{T}}$ & 0.002 & 0.00215 & $\mathrm{~N} / \mathrm{A}$ & $\mathrm{N} / \mathrm{A}$ & $\mathrm{N} / \mathrm{A}$ & $\mathrm{N} / \mathrm{A}$ \\
\hline & \multirow{4}{*}{ Numerical } & $K_{T}$ & 0.121 & 0.09840 & 0.2054 & 0.052 & 0.2239 & 0.2617 \\
\hline & & $u_{K_{T}}$ & 0.005 & 0.00330 & 0.0049 & 0.003 & 0.0099 & 0.0083 \\
\hline & & $\mathrm{E}$ & 0.001 & -0.00930 & 0.0221 & 0.005 & 0.0071 & -0.0180 \\
\hline & & $u_{v}$ & 0.005 & 0.00394 & 0.0049 & 0.003 & 0.0099 & 0.0083 \\
\hline \multirow{6}{*}{$J_{H}$} & \multirow{2}{*}{ Experimental } & $K_{T}$ & 0.285 & 0.31730 & 0.3947 & 0.193 & 0.4751 & 0.6631 \\
\hline & & $u_{K_{T}}$ & 0.006 & 0.00635 & $\mathrm{~N} / \mathrm{A}$ & $\mathrm{N} / \mathrm{A}$ & $\mathrm{N} / \mathrm{A}$ & $\mathrm{N} / \mathrm{A}$ \\
\hline & \multirow{4}{*}{ Numerical } & $K_{T}$ & 0.265 & 0.24190 & 0.3855 & 0.171 & 0.4924 & 0.5226 \\
\hline & & $u_{K_{T}}$ & 0.003 & 0.00156 & 0.0039 & 0.003 & 0.0104 & 0.0024 \\
\hline & & $\mathrm{E}$ & -0.020 & -0.07540 & -0.0092 & -0.022 & 0.0173 & -0.1405 \\
\hline & & $u_{v}$ & 0.007 & 0.00654 & 0.0039 & 0.003 & 0.0104 & 0.0024 \\
\hline \multirow{6}{*}{$J_{H}$} & \multirow{2}{*}{ Experimental } & $K_{T}$ & 0.146 & 0.16250 & 0.2147 & 0.095 & 0.1616 & 0.2082 \\
\hline & & $u_{K_{T}}$ & 0.003 & 0.00325 & $\mathrm{~N} / \mathrm{A}$ & $\mathrm{N} / \mathrm{A}$ & $\mathrm{N} / \mathrm{A}$ & $\mathrm{N} / \mathrm{A}$ \\
\hline & \multirow{4}{*}{ Numerical } & $K_{T}$ & 0.148 & 0.14410 & 0.2340 & 0.094 & 0.1671 & 0.1984 \\
\hline & & $u_{K_{T}}$ & 0.005 & 0.00271 & 0.0048 & 0.003 & 0.0098 & 0.0092 \\
\hline & & $\mathrm{E}$ & 0.002 & -0.01840 & 0.0193 & -0.001 & 0.0055 & -0.0098 \\
\hline & & $u_{v}$ & 0.005 & 0.00423 & 0.0048 & 0.003 & 0.0098 & 0.0092 \\
\hline
\end{tabular}

Source: Author

From the qualitative observations and tables 9 and 10, the following following conclusions can be drawn for the PLL:

- for most of the propellers assessed, the $K_{T} \times J$ curves presented a common trend: the use of Star-CCM+ (or Brockett's expressions, when applicable) as the source of 2-D improved the accuracy of the formulation, and the PLL-TFT combination usually overestimated this coefficient; still, as in the wing case, the question about whether the time spent to generate a database must be answered; 
Table 10 - Validation for the Propeller Lifting-Line formulation. Torque Coefficient

\begin{tabular}{|c|c|c|c|c|c|c|c|c|}
\hline & Propeller & & P4119 & P4133 & $\mathrm{P} 4381$ & $\mathrm{H} 1$ & MOD5 & PPTC \\
\hline \multirow{6}{*}{$J_{L}$} & \multirow{2}{*}{ Experimental } & $K_{Q}$ & 0.0106 & 0.01366 & 0.03054 & 0.0056 & 0.03727 & 0.04944 \\
\hline & & $u_{K_{Q}}$ & 0.0002 & 0.00027 & $\mathrm{~N} / \mathrm{A}$ & $\mathrm{N} / \mathrm{A}$ & $\mathrm{N} / \mathrm{A}$ & $\mathrm{N} / \mathrm{A}$ \\
\hline & \multirow{4}{*}{ Numerical } & $K_{Q}$ & 0.0110 & 0.01630 & 0.03670 & 0.0042 & 0.03365 & 0.05380 \\
\hline & & $u_{K_{Q}}$ & 0.0013 & 0.00106 & 0.00151 & 0.0006 & 0.00226 & 0.00348 \\
\hline & & $\mathrm{E}$ & 0.0004 & 0.00264 & 0.00616 & -0.0014 & -0.00362 & 0.00436 \\
\hline & & $u_{v}$ & 0.0013 & 0.00110 & 0.00151 & 0.0006 & 0.00226 & 0.00348 \\
\hline \multirow{6}{*}{$J_{M}$} & \multirow{2}{*}{ Experimental } & $K_{Q}$ & 0.0239 & 0.02586 & 0.04085 & 0.0121 & 0.04676 & 0.07676 \\
\hline & & $u_{K_{Q}}$ & 0.0005 & 0.00052 & $\mathrm{~N} / \mathrm{A}$ & $\mathrm{N} / \mathrm{A}$ & $\mathrm{N} / \mathrm{A}$ & $\mathrm{N} / \mathrm{A}$ \\
\hline & \multirow{4}{*}{ Numerical } & $K_{Q}$ & 0.0262 & 0.02750 & 0.04650 & 0.0108 & 0.04372 & 0.08040 \\
\hline & & $u_{K_{Q}}$ & 0.0013 & 0.00103 & 0.00159 & 0.0008 & 0.00243 & 0.00360 \\
\hline & & $\mathrm{E}$ & 0.0023 & 0.00164 & 0.00565 & -0.0013 & -0.00304 & 0.00364 \\
\hline & & $u_{v}$ & 0.0014 & 0.00115 & 0.00159 & 0.0008 & 0.00243 & 0.00360 \\
\hline \multirow{6}{*}{$J_{H}$} & \multirow{2}{*}{ Experimental } & $K_{Q}$ & 0.0447 & 0.05790 & 0.07048 & 0.0281 & 0.07679 & 0.14650 \\
\hline & & $u_{K_{Q}}$ & 0.0009 & 0.00116 & $\mathrm{~N} / \mathrm{A}$ & $\mathrm{N} / \mathrm{A}$ & $\mathrm{N} / \mathrm{A}$ & $\mathrm{N} / \mathrm{A}$ \\
\hline & \multirow{4}{*}{ Numerical } & $K_{Q}$ & 0.0490 & 0.05030 & 0.07540 & 0.0259 & 0.06796 & 0.12680 \\
\hline & & $u_{K_{Q}}$ & 0.0016 & 0.00107 & 0.00194 & 0.0009 & 0.00371 & 0.00407 \\
\hline & & $\mathrm{E}$ & 0.0043 & -0.00760 & 0.00492 & -0.0022 & -0.00883 & -0.01970 \\
\hline & & $u_{v}$ & 0.0019 & 0.00158 & 0.00194 & 0.0009 & 0.00371 & 0.00407 \\
\hline \multirow{6}{*}{$J_{D}$} & \multirow{2}{*}{ Experimental } & $K_{Q}$ & 0.0280 & 0.03409 & 0.04465 & 0.0179 & 0.03897 & 0.06300 \\
\hline & & $u_{K_{Q}}$ & 0.0006 & 0.00068 & $\mathrm{~N} / \mathrm{A}$ & $\mathrm{N} / \mathrm{A}$ & $\mathrm{N} / \mathrm{A}$ & $\mathrm{N} / \mathrm{A}$ \\
\hline & \multirow{4}{*}{ Numerical } & $K_{Q}$ & 0.0306 & 0.03440 & 0.05130 & 0.0161 & 0.03545 & 0.06780 \\
\hline & & $u_{K_{Q}}$ & 0.0014 & 0.00102 & 0.00163 & 0.0008 & 0.00240 & 0.00354 \\
\hline & & $\mathrm{E}$ & 0.0026 & 0.00031 & 0.00665 & -0.0018 & -0.00352 & 0.00480 \\
\hline & & $u_{v}$ & 0.0015 & 0.00122 & 0.00163 & 0.0008 & 0.00240 & 0.00354 \\
\hline
\end{tabular}

Source: Author

- in most of the cases, the agreement between experimental and numerical data happened when the assumption about the moderately loaded condition seemed satisfied; such condition could be observed by the plots of the ratio of the induced by the advance and rotational velocities; the $C_{T}$ was another metric used to characterize loading conditions, but unlike the velocity case, the results obtained from $C_{T}$ seem more inconclusive, as this variable significantly varied in terms of order of magnitude for one propeller to another, and the agreement did not seem to explicitly depend on such magnitude;

- the influence of the geometry also plays a role on the accuracy of the PLL: despite the skew distribution, one of the possible reasons for satisfactory agreement of MOD5 propeller is its large AR, while the low AR of $\mathrm{P} 4133$ is possibly one of the main reasons for the discrepancies observed (as P4119 agreed better with the data);

- as in the wing case, the conclusions about $K_{Q}$ are unclear: depending on $J$ and the propeller geometry and flow regime, the use of 2-D data-enhanced, was indifferent to 
or diminished the accuracy in comparison to the experimental data. Such behavior might have happened due to the strong dependence of $C_{c}$ on Reynolds and the 3-D effects of the flow. Probably, the use of a more accurate database could increase the results for $K_{Q}$ as well.

- table 9 shows that - by taking $E=10 u_{K_{T}}$ as the adopted "much larger" criterion on equation (3.40), for $J_{L}$, more than $65 \%$ of the data falls within the noise level, while for $J_{M}$ this percentage is higher than $35 \%$ and for $J_{D}$ this figure is $50 \%$, according to equation (3.41). This is expected due to the model used, and such percentages are likely to be improved with the improvement of the source of 2-D data. In terms of $J_{H}$, no data falls within noise level, but rather a few indicates that the model could be improved; this is also expected because, as a general trend, the decrease in $J$ makes the discrepancies between numerical and experimental data increase. So, in addition to the improvement of $2-\mathrm{D}$ data, decreasing $J$ would indicate the inadequacy of the method at heavily loading conditions.

- the data for $K_{Q}$ presented on table 10 shows a worse agreement than $K_{T}$, meaning that errors are higher for this variable: only for lightly and design advance ratios, there are a few data that indicate the error within noise level. A possible explanation is how the $C_{D}$ has been approached in the propeller case, as the blade sections are likely under different (often more severe, i.e., more drag-imposing) conditions than the wing sections. So, the inaccuracy of the numerical foil section data, the extrapolation of these data for high angles of attack, and incapacity of the method to model heavily loading conditions contribute to the discrepancies.

- still, for engineering purposes, as a preliminary analysis tool the proposed PLL seems suitable, as for a significant number of cases the numerical $K_{T}$ data agreed with experimental when the moderately loaded condition was satisfied (which was also near design advance coefficient). Additionally, it is important to note the contribution of this formulation to the inclusion of viscosity on the hydrodynamic coefficients and, equally important, to the expanded capability on simulating skewed and raked propellers: they evidence the enhancement of the classic formulation for the analysis of more geometrically-general propellers. 


\section{Conclusion and Future Work}

\subsection{Conclusion}

This work introduces a literature review on the progress of the lifting-line theories for wings and propellers since their early conception. The review shows the extensions that the wing formulation has gone through to be capable of simulating arbitrary geometries under viscous flow conditions, and the adaptations of the propeller counterpart to make more accurate designs of moderately loaded propellers possible.

A novel formulation of the wing lifting-line, essentially based on vector algebra, is presented. It enables the simulation of general wing geometries under viscous flows through the use of the Pistolesi Boundary Condition in a nonlinear scheme that considers information about real foil data. With proper adaptations, the formulation is extended to the propeller problem, and the outcome as expected is a formulation that enables the simulation of general propeller geometries under the moderately loaded condition.

The assessment of the wing lifting-line showed that, in terms of code and solution verification, cosine clustering is preferred over the equidistant one, as for all the cases studied the former presented adequate rates of convergence and mesh uncertainties, while the latter presented poor convergence behavior and significantly greater uncertainties. Additionally, these results indicate associated modeling errors as illustrated by the differences between numerical and theoretical results. Comparison with experimental data showed that regardless of the case, the discrepancies between numerical and experimental $C_{L}$ increase with the increase in the incidence angle of attack. It also showed that this agreement is better whenever real 2-D data is used. Regarding $C_{D}$, conclusions are unclear and a deeper study about the influence of Reynolds on the lifting-line could improve such conclusions.

The assessment of the propeller lifting-line showed practically the same trends observed in the wing case; code and solution verification showed the advantages of the cosine clustering over the equidistant one, in addition to the appropriateness of the proposed numerical wake model and its associated discretization. Comparison with experimental data showed that for most of the cases the agreement between experimental and numerical $K_{T}$ data happened when the assumption about the moderately loaded condition seemed satisfied, and the use of real 2-D data improved its accuracy. It also seems that blade geometry influences the accuracy of the method. Finally, as in the wing case, the conclusions about $K_{Q}$ are unclear, and deeper study about the influence of Reynolds on the lifting-line could improve such conclusions. 


\subsection{Future Work}

\subsubsection{PLL-CFD Coupled Methods}

According to the $27^{\text {th }}$ ITTC Propulsion Committee, there has been an inclination towards coupling simpler and more sophisticated fluid dynamics methods in propeller design to reduce computation time while keeping reasonable numerical accuracy; one example is the coupling of LLT and CFD methods to simulate several problems of interest, such as counter-rotating propellers, hull-propellers, and ducted propellers. Although there is an apparent trend on how to do such coupling - by using the actuator disk model as an interface between the LLT and CFD -, there is not to the present date a well-defined systematic approach - as the inputs and outputs exchanged through the actuator disk, the appropriate number of layers to model it, and the conversation time. Certainly, whatever the best approach is, it can benefit from the proposed PLL and its expanded capabilities i.e., the generality of propeller geometries, the inclusion of viscosity, and the speed and proven accuracy near design conditions; a future plane is to explore approaches to the LLT-CFD coupling while using the developed PLL formulation.

\subsubsection{Hydroelastic Propellers}

The same Propulsion Committee recommended the use of multidisciplinary optimization techniques to maximize propeller design, as they present gains over the traditional spiral design procedures. Also, there has been an increasing interest from the marine industry in the use of composite materials to improve the hydrodynamic and structural performance of marine propellers, as these materials, more than just cheaper than the traditional, present high strength-to-weight and stiffness-to-weight ratios, so their anisotropic characteristic can be explored to favor strains over preferred directions thus reducing loads and stresses and improving performance by passively adjusting the propeller rake, skew, and pitch distributions. This hydroelastic interaction can be modeled through coupled methods like the one presented in this text and a finite-element-based for the structural part. Multidisciplinary optimization for flexible propeller design represents a field of study that can lead to expressive gains in pollutant emission reduction as well, and the advantages of the present lifting-lines can be used to such purposes - in particular, because it is relatively easy and fast to generate $K_{T} \times J$ and $K_{Q} \times J$ curves.

\subsubsection{General Changes in the Propeller Formulation}

The novel propeller approach has proven powerful, but both its results and capabilities can be expanded; as addressed through the text, the inclusion of free-wake and more robust hub models can improve the results near heavily loading conditions. Another possibility is to take advantage of the already existing source of 2 -D data to include 
cavitation inception through the inspection of the minimum $C_{P}$ distribution throughout the blades. Those are relatively simple changes that already can have a positive impact on the PLL. 



\section{Bibliography}

ANDERSON, J. D.; CORDA, S.; WIE, D. M. V. Numerical lifting line theory applied to drooped leading-edge wings below and above stall. Journal of Aircraft, v. 17, n. 12, p. 898-904, 1980. Disponível em: <https://doi.org/10.2514/3.44690>. Cited on page 26.

APPLIN, Z. T. Pressure distributions from subsonic tests of a NACA 0012 semispan wing model. [S.l.], 1995. v. 110148, n. 110148. Cited 2 times on pages 76 and 79.

ASME. Standard for verification and validation in computational fluid dynamics and heat transfer. New York, U.S.A, 2009. v. 2000. Cited on page 55.

BARKMANN, U. Potsdam Propeller Test Case (PPTC) - Open Water Tests with the Model Propeller VP1304. [S.1.], 2011. Disponível em: <https://www.sva-potsdam.de/ wp-content/uploads/2016/04/SVA_report_3752.pdf $>$. Cited 2 times on pages 94 and 107.

BERTRAM, V. Practical Ship Hydrodynamics. Second edition. Oxford: ButterworthHeinemann, 2012. 333 - 365 p. ISBN 978-0-08-097150-6. Disponível em: <http: //www.sciencedirect.com/science/article/pii/B9780080971506100119>. Cited on page 18.

BERTSCHNEIDER, H. et al. Final report and Recommendations to the 27th ITTC. [S.l.], 2014. 45 p. Cited 2 times on pages 17 and 18.

BOSWELL, R. J. Design, cavitation performance, and open-water performance of a series of research skewed propellers. [S.1.], 1971. Disponível em: <https: //apps.dtic.mil/dtic/tr/fulltext/u2/732511.pdf>. Cited on page 100.

BROCKETT, T. Minimum Pressure Envelopes for Modified Naca-66 Sections with Naca $a=0.8$ Camber and Buships Type 1 And Type 2 Sections. [S.1.], 1966. Disponível em: $<$ https://apps.dtic.mil/dtic/tr/fulltext/u2/629379.pdf $>$. Cited 4 times on pages 48, 49, 53 , and 92 .

CAJA, A. S. On the Optimum Propeller Loading with Inclusion of Duct and Hub. Dissertação (Mestrado) - Massachusetts Institute of Technology, Department of Ocean Engineering, 1988. Cited on page 43.

CANTWELL, B. J. Wings of finite span. [S.1.]: Stanford University Department of Aeronautics and Astronautics, 2013. < https://web.stanford.edu/ cantwell/AA200_ Course_Material/AA200_Course_Notes/AA200_Ch_12_Wings_of_Finite_Span_ Cantwell.pdf $>$. Cited on page 68.

CARLTON, J. Marine propellers and propulsion. Fourth edition. ButterworthHeinemann, 2019. 1-585 p. ISBN 978-0-08-100366-4. Disponível em: <http: //www.sciencedirect.com/science/article/pii/B9780081003664099855>. Cited 7 times on pages 17, 18, 19, 28, 29, 33, and 49 .

CHREIM, J. R. et al. Viscous effects assessment through nonlinear lifting-line theory. In: ABCM - ASSOCIACAO BRASILEIRA DE ENGENHARIA E CIENCIAS MECANICAS. 24th ABCM International Congress of Mechanical Engineering. [S.1.], 2017. Cited 3 times on pages 27,82 , and 125 . 
CHUNG, H.-L. An enhanced propeller design program based on propeller vortex lattice lifting line theory. Dissertação (Mestrado) - Massachusetts Institute of Technology, jun. 2007. Disponível em: <https://dspace.mit.edu/handle/1721.1/39729>. Cited 2 times on pages 30 and 40 .

DAM, C. P. van; VIJGEN, P. M. H. W.; HOLMES, B. J. Experimental investigation on the effect of crescent planform on lift and drag. Journal of Aircraft, v. 28, n. 11, p. 713-720, 1991. Disponível em: <https://doi.org/10.2514/3.46087>. Cited 2 times on pages 76 and 78 .

DENNY, S. B. Cavitation and open-water performance tests of a series of propellers designed by lifting-surface methods. [S.1.], 1968. Disponível em: < https: //apps.dtic.mil/dtic/tr/fulltext/u2/841845.pdf $>$. Cited 5 times on pages 48, 49, 91, 94, and 97 .

DINIZ, G. A fully numerical lifting line method for the design of heavily loaded marine propellers with rake and skew. Dissertação (Mestrado) - Massachusetts Institute of Technology, jun. 2015. Disponível em: < https://dspace.mit.edu/handle/1721.1/100140>. Cited 2 times on pages 40 and 41 .

DRAGOS, L. a. Mathematical methods in aerodynamics. 1. ed. [S.1.]: Springer Netherlands, 2004. ISBN 978-1-4020-1663-9. Cited 2 times on pages 23 and 26.

DRELA, M.; YOUNGREN, H. Xfoil: An analysis and design system for low reynolds number airfoils. In: SPRINGER. Low Reynolds Number Aerodynamics. [S.l.]: Springer, Berlin, Heidelberg, 1989. ISBN 978-3-540-51884-6. Cited on page 81.

ECA, L.; HOEKSTRA, M. A procedure for the estimation of the numerical uncertainty of cfd calculations based on grid refinement studies. Journal of Computational Physics, v. 262, p. 104 - 130, 2014. ISSN 0021-9991. Disponível em: <http://www.sciencedirect.com/science/article/pii/S0021999114000278>. Cited 3 times on pages 50,61, and 62 .

EPPS, B. On the rotor lifting line wake model. Journal of Ship Production and Design, Society of Naval Architects and Marine Engineers (SNAME), v. 33, n. 1, p. 31-45, 2017. Cited 6 times on pages 31, 32, 42, 43, 44, and 91 .

EPPS, B. et al. Openprop: An open-source parametric design and analysis tool for propellers. In: SOCIETY FOR MODELING \& SIMULATION INTERNATIONAL. Proceedings of the 2009 grand challenges in modeling 86 simulation conference. [S.l.], 2009. p. 104-111. Cited on page 49.

EPPS, B.; KIMBALL, R. OpenProp v3: Open-source software for the design and analysis of marine propellers and horizontal-axis turbines. 2013. Disponível em: $<$ http://engineering.dartmouth.edu/epps/openprop $>$. Cited on page 31.

EPPS, B. P. An impulse framework for hydrodynamic force analysis: fish propulsion, water entry of spheres, and marine propellers. Tese (Doutorado) — Massachusetts Institute of Technology, fev. 2010. Disponível em: <https://dspace.mit.edu/handle/1721.1/61519>. Cited on page 31 . 
EPPS, B. P.; KIMBALL, R. W. Unified rotor lifting line theory. Journal of ship research, Society of Naval Architects and Marine Engineers (SNAME), v. 57, n. 4, p. 181-201, 2013. Cited on page 67.

ESTEVES, F. R. et al. Simulation of darpa suboff model propeller hull interaction. In: Proceedings of the 27 th International Congress on Waterborne Transportation, Shipbuilding and Offshore Constructions. [S.1.: s.n.], 2018. Cited on page 105.

FLOOD, K. M. Propeller performance analysis using lifting line theory. [S.l.], 2009. Cited 3 times on pages 18,30 , and 40 .

GALLAY, S.; GHASEMI, S.; LAURENDEAU, E. Sweep effects on non-linear lifting line theory near stall. In: 52nd Aerospace Science Meeting. [S.l.: s.n.], 2014. p. 2014-1105. Cited on page 27.

GHOSE, J. Basic ship propulsion. [S.l.]: Allied publishers, 2004. Cited 2 times on pages 48 and 49.

GROVES, N. C.; HUANG, T. T.; CHANG, M. S. Geometric characteristics of DARPA (Defense Advanced Research Projects Agency) SUBOFF models (DTRC model numbers 5470 and 5471). [S.l.], 1989. Cited on page 105.

HALL, N. Inclination Effects on Lift. 2018. <https://www.grc.nasa.gov/www/k-12/ airplane/incline.html>. Accessed: 2020-02-26. Cited on page 83.

HUNSAKER, D. F. A numerical lifting-line method using horseshoe vortex sheets. Utah State University. 2011. Cited 2 times on pages 27 and 41.

The Propulsion Commiittee: Final Report and Recommendations to the 28th ITTC. Cited on page 17.

JESSUP, S. D. An experimental investigation of viscous aspects of propeller blade flow. Tese (Doutorado) - Massachusetts Institute of Technology, 1989. Cited 2 times on pages 91 and 94.

JR, J. D. A. Fundamentals of aerodynamics. 5. ed. [S.l.]: McGraw-Hill Education, 2010. ISBN 9780073398105. Cited on page 23.

JR, J. W. The Calculation of Propeller Induction Factors AML Problem 69-54. [S.1.], 1957. Cited 2 times on pages 29 and 88.

KATSUNO, E.; DANTAS, J. Analysis of the blockage effect on a cavitation tunnel using cfd tools. In: ASME 2017 38th International Conference on Ocean, Offshore and Arctic Engineering, American Society of Mechanical Engineers. [S.l.: s.n.], 2017. Cited 3 times on pages 94, 102, and 105.

KATZ, J.; PLOTKIN, A. Low-speed aerodynamics. [S.l.]: Cambridge University Press, 2001. v. 13. Cited 15 times on pages 21,22 , 23, 24, 25, 26, 34, 35, 36, 42, 48, 67, 96, 126, and 128.

KAWADA, S. Induced velocity by helical vortices. J. Aeronaut. Sci, 1936. Cited 2 times on pages 28 and 40 . 
KERWIN, J.; LEOPOLD, R. A desing theory for subcavitating propellers. In: . [S.l.: s.n.], 1964. Cited 2 times on pages 29 and 42 .

KERWIN, J. E. The Solution of Propeller Lifting Surface Problems by Vortex Lattice Methods. Tese (Doutorado) — Massachusetts Institute of Technology, 1961. Cited on page 91 .

KERWIN, J. E. Computer techniques for propeller blade section design. International Shipbuilding Progress, IOS Press, v. 20, n. 227, p. 227-251, 1973. Cited on page 18.

KERWIN, J. E.; HADLER, J. B. Principles of naval architecture series: Propulsion. The Society of Naval Architects and Marine Engineers (SNAME), 2010. Cited 7 times on pages $18,30,32,42,67,88$, and 102 .

KUETHE, A. M.; CHOW, C.-Y. Foundations of aerodynamics: bases of aerodynamic design. 5th. ed. [S.1.]: J. Wiley, 1997. ISBN 978-0-471-12919-6. Cited on page 25.

LERBS, H. W. Moderately loaded propellers with a finite number of blades and arbitrary distribution of circulation. Trans SNAME, 1952. Cited 2 times on pages 29 and 43.

MARIN. Propeller design with induction factors. 2010. < http://citeseerx.ist.psu.edu/ viewdoc/download?doi=10.1.1.720.5452\&rep=rep1\&type=pdf $>$. Cited on page 32 .

MORGAN, W. B.; SILOVIC, V.; DENNY, S. B. Propeller lifting-surface corrections. [S.l.], 1968. Cited 2 times on pages 18 and 29.

ORTEGA, M. A.; GIRARDI, R. M.; KOMATSU, P. S. A numerical method to predict the lift of aircraft wings at stall conditions. In: ABCM - ASSOCIACAO BRASILEIRA DE ENGENHARIA E CIENCIAS MECANICAS. 10th Brazilian Congress of Thermal Engineering and Sciences. [S.l.], 2004. Cited on page 27.

OWENS, D. B. Weissinger's model of the nonlinear lifting-line method for aircraft design. In: 36th Aerospace Sciences Meeting and Exhibit AIAA Paper. [S.l.: s.n.], 1998. v. 597. Cited on page 27.

PEPPER, R. S.; DAM, C. van. Design methodology for multi-element high-lift systems on subsonic civil transport aircraft. [S.l.], 1996. Cited 2 times on pages 27 and 34.

PHILLIPS, W.; SNYDER, D. Modern adaptation of prandtl's classic lifting-line theory. Journal of Aircraft, v. 37, n. 4, p. 662-670, 2000. Cited 6 times on pages 27, 34, 36, 67, 125 , and 129.

PISTOLESI, E. Considerations respecting the mutual influence of systems of airfoils. In: Collected Lectures of the 1937 Principal Meeting of the Lilienthal Society. [S.l.: s.n.], 1937. Cited 2 times on pages 26 and 44 .

SAFFMAN, P. G. Vortex dynamics. [S.l.]: Cambridge university press, 1992. Cited on page 23 .

SANCHEZ-CAJA, A. Dtrc propeller 4119 calculations at vtt. In: Proceedings of the 22nd ITTC propulsion committee propeller RANS/panel method workshop, Grenoble, France. [S.l.: s.n.], 1998. Cited on page 91. 
Silva Jr, H. C. et al. Experimental and numerical analysis of tip vortex of a darpa suboff auv propeller. In: Proceedings of the 14th Practical Design of Ships and Other Floating Structures - PRADS 2019 (Expected). Yokohama, Japan: [s.n.], 2019. Cited 2 times on pages 94 and 105.

SOUZA, S. L. de. Elaboração de uma Metodologia para predição do Coeficiente de Sustentacao Maximo de Asas Flapeadas. Dissertação (Mestrado) — Instituto de Tecnológico de Aeronáutica, 2005. Cited on page 34.

STUBBLEFIELD, J. M. Numerically-based ducted propeller design using vortex lattice lifting line theory. Tese (Doutorado) - Massachusetts Institute of Technology, 2008.

Cited on page 30 .

TIAN, Y.; KINNAS, S. A wake model for the prediction of propeller performance at low advance ratios. International Journal of Rotating Machinery, v. 2012, 08 2012. Cited on page 41 .

VAZ, G.; ECA, L.; TOXOPEUS, S. Numerical Uncertainty Analysis. 2018. $<$ https://www.refresco.org/verification-validation/utilitiesvv-tools/>. Cited on page 50 .

VAZ, G. N. V. B. Modelling of sheet cavitation on hydrofoils and marine propellers using boundary element methods. Tese (Dissertation) - Instituto Superior Tecnico, Lisbon, Portugal, November 2005. Cited on page 19.

WANG, M.-H. Hub effects in propeller design and analysis. [S.l.], 1985. Cited 2 times on pages 30 and 47 .

WEBER, J.; BREBNER, G. Low-Speed Tests on 45-deg Swept-Back Wings. [S.1.], 1951. Cited 2 times on pages 76 and 81 .

WEISSINGER, J. The lift distribution of swept-back wings. [S.l.], 1947. Cited on page 26.

WICKENHEISER, A. M.; GARCIA, E. Aerodynamic modeling of morphing wings using an extended lifting-line analysis. Journal of Aircraft, v. 44, n. 1, p. 10-16, 2007. Disponível em: <https://doi.org/10.2514/1.18323>. Cited on page 33 . 

Appendix 



\section{APPENDIX A - Necessary Mathematical and Physical Derivations}

\section{A.1 Reasoning for keeping part of the HSV over the wing/blade planforms}

The appropriate choice of the HSV is essential in the LLT as this interferes with the applicability and even convergence of the numerical method. The most common options are presented on figure 74 .

Figure 74 - Possible HSV models. a) TV directly shed into the free-stream from the BV; b) Part of the TV remains over the planform, while the other part is shed into the free-stream. c) The TV is shed into the planform plane direction.

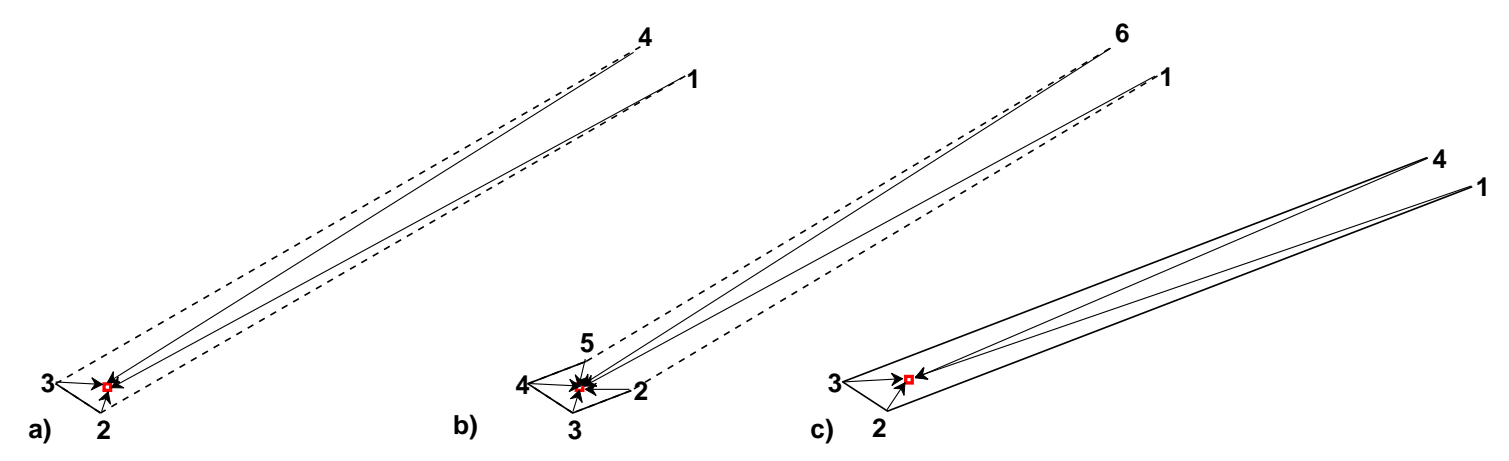

Source: Author

The HSV 'a' was initially adopted and used on previous works (CHREIM et al., 2017). Although suitable for formulations in which the CP is placed over the BV(PHILLIPS; SNYDER, 2000), this geometry does not work properly when the PBC must be satisfied: the circulation distribution becomes unstable and leads to divergence as the mesh is refined (Figure 75). Such instability can be physically explained with the aid of figure 76, which presents the respective parts of the HSVs 'a' and ' $b$ ' that remain over the wing planform. For the sake of explanation, the HSVs have unitary circulation and the induced velocities are qualitatively represented by the numbered arrows: on a) the velocities induced by the segments over the spanwise outermost CPs are lower than over the innermost, implying 
that the final $\Gamma$ distribution is higher at the tips. This effect increases with the AoA, as the free trailing vortices become less influential; if instead, a trailing leg extends from the quarter chord-line to the trailing edge on each HSV, as on 'b', an additional contribution makes the velocity induced at the tips highly increase, while the increase near the root is not as intense. This makes $\Gamma$ decreases towards the tips and improves numerical stability (Figure 77).

Figure 75 - Behavior of $\Gamma$ as $N$ is increased for the HSV type ' $a$ '.

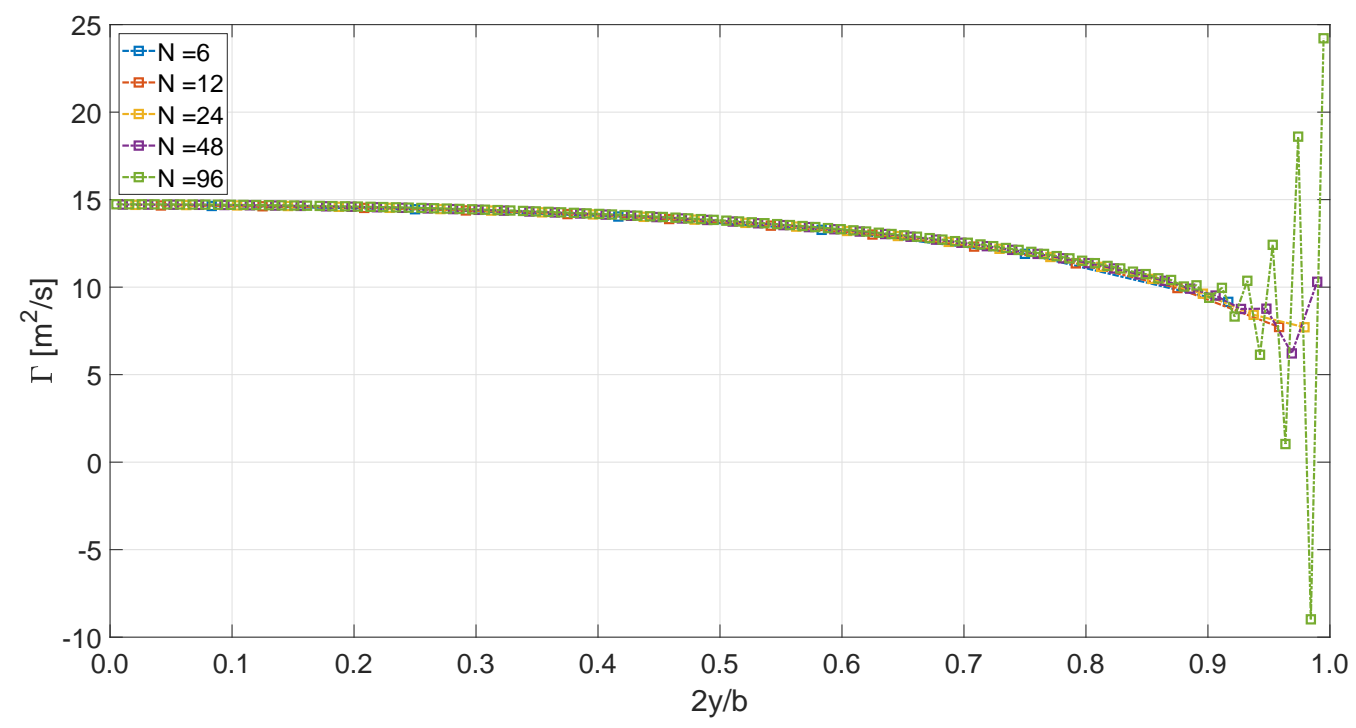

Source: Author

Therefore, between HSVs ' $a$ ' and 'b', the second is more appropriate. In comparison to the HSV ' $c$ ', while there is no such straightforward advantage in using one over another, an important consideration about the physics of the flow is that the trailing vortices must be shed into the flow towards the free-stream direction so that no aerodynamic forces act on them (KATZ; PLOTKIN, 2001). Although for very small $\alpha$ such consideration can be assumed to be true for the HSV ' $c$ ', as the angle of attack increases this assumption can no longer be valid. Therefore, to correctly satisfy the physics behind the formulation, the HSV 'b' was chosen as the singularity element. 
Figure 76 - Parts of the HSVs that stay on the wing planform regardless of the AoA. The arrows (from left to right) represent the induced velocities of the HSVs (from top to down), with the as their magnitude at the specific CP (1 is the strongest).
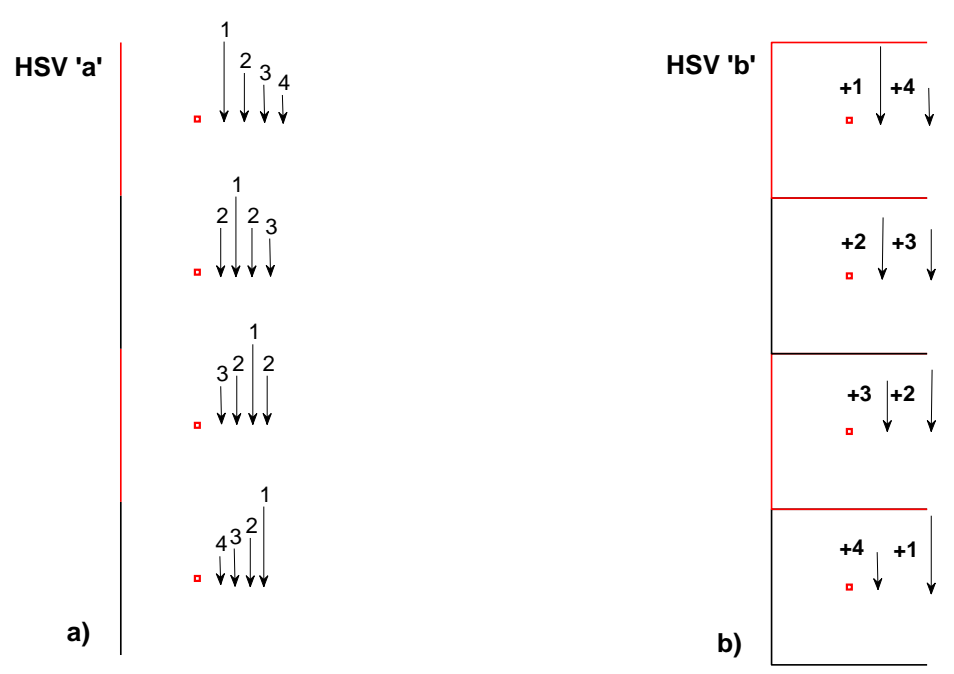

Source: Author

Figure 77 - Behavior of $\Gamma$ as $N$ is increased for the HSV type 'b'.

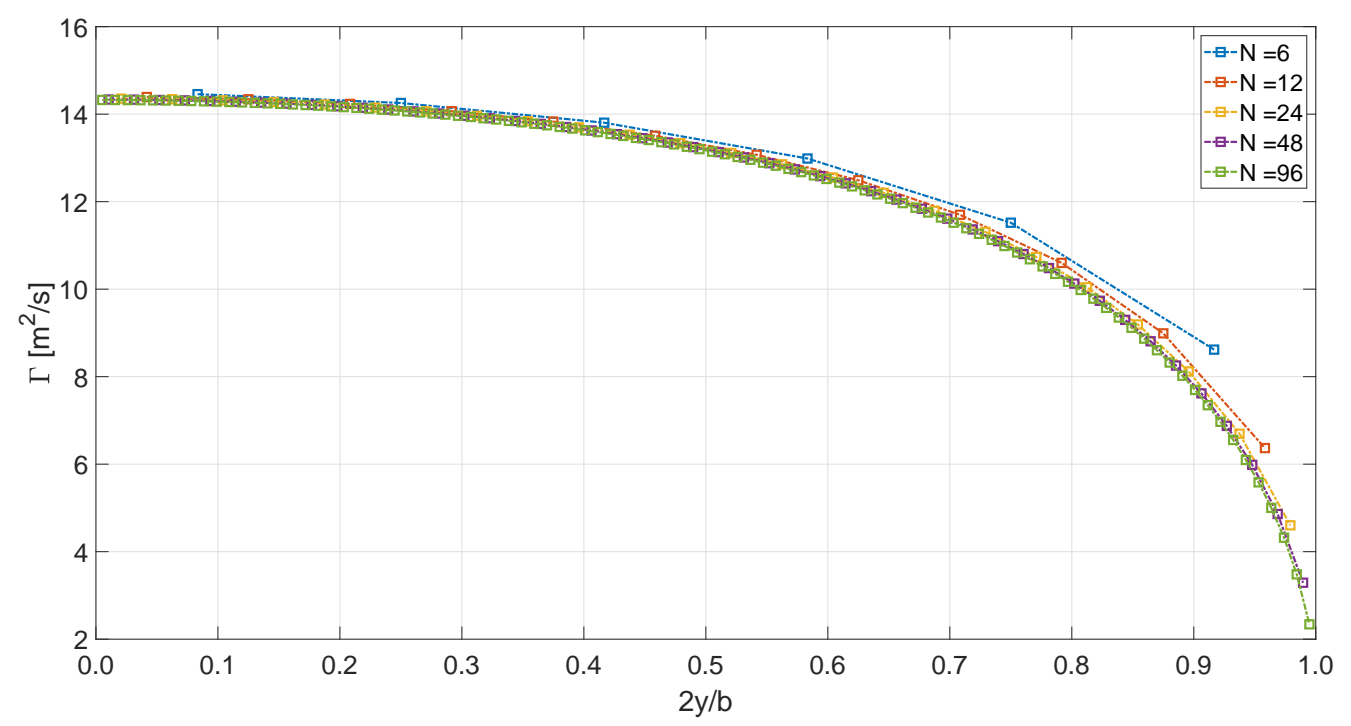

Source: Author

\section{A.2 Velocity induced by a straight vortex segment}

Consider a straight vortex segment of length $\delta \vec{l}$ and strength $\Gamma$, as in figure 78 ; according to the Biot-Savart's law for aerodynamics, the velocity induced $d \vec{V}_{V S}$ by an infinitesimal part $d \vec{l}$ of this segment at an arbitrary point $P$ in space, whose distance is $\vec{r}$, 
is:

$$
d \vec{V}_{V S}=\frac{\Gamma}{4 \pi} \frac{d \vec{l} \times \vec{r}}{r^{3}}
$$

Figure 78 - Velocity induced by a straight segment vortex.

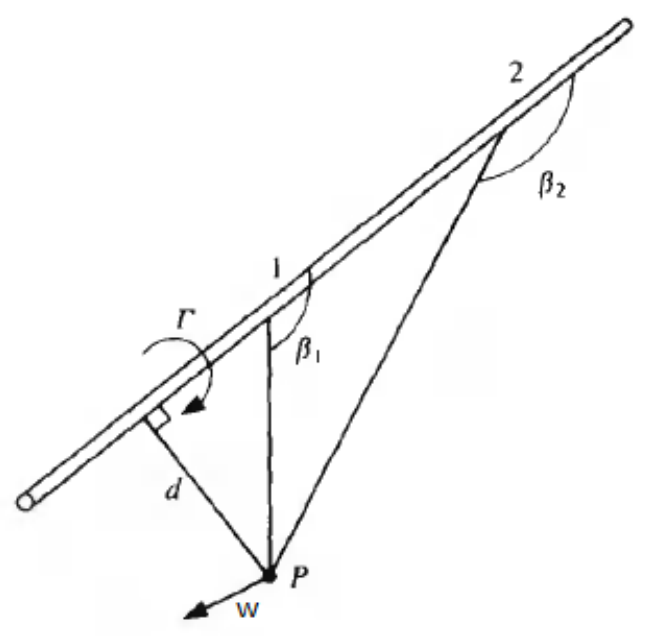

Source: KATZ; PLOTKIN, 2001

The magnitude of the induced velocity $V_{V S}$ of the entire segment in terms of the distance $H$ and the angles $\phi_{1}$ and $\phi_{2}$ is obtained integrating the last expression:

$$
V_{V S}=\frac{\Gamma}{4 \pi H}\left(\cos \phi_{1}-\cos \phi_{2}\right)
$$

These parameters can be rewritten in terms of the length vectors $\vec{r}_{1}$ and $\vec{r}_{2}$ that connect the left and right edges, respectively, to point $P$, as in figure 17):

$$
\begin{aligned}
H & =\frac{\left|\vec{r}_{1} \times \vec{r}_{2}\right|}{\left|\vec{r}_{2}-\vec{r}_{1}\right|} \\
\cos \phi_{1} & =\frac{\left(\vec{r}_{2}-\vec{r}_{1}\right) \cdot \vec{r}_{1}}{\left|\vec{r}_{2}-\vec{r}_{1}\right| r_{1}} \\
\cos \phi_{2} & =\frac{\left(\vec{r}_{2}-\vec{r}_{1}\right) \cdot \vec{r}_{2}}{\left|\vec{r}_{2}-\vec{r}_{1}\right| r_{2}}
\end{aligned}
$$

The direction of $\vec{V}_{V S}$ is normal to the plane created by point $\mathrm{P}$ and the vortex edges:

$$
\hat{V}_{V S}=\frac{\vec{r}_{1} \times \vec{r}_{2}}{\left|\vec{r}_{1} \times \vec{r}_{2}\right|}
$$


Substituting these quantities on Eq. (A.2) and multiplying the expression by the normal vector $\hat{V}_{V S}$, the induced velocity vector $\vec{V}_{V S}$ is obtained:

$$
\vec{V}_{V S}=\frac{\Gamma}{4 \pi} \frac{\vec{r}_{1} \times \vec{r}_{2}}{\left|\vec{r}_{1} \times \vec{r}_{2}\right|^{2}}\left(\vec{r}_{2}-\vec{r}_{1}\right) \cdot\left(\frac{\vec{r}_{1}}{r_{1}}-\frac{\vec{r}_{2}}{r_{2}}\right)
$$

Equation (A.7), as is, is not useful for numerical calculations, as it is singular whenever $\vec{r}_{1}$ and $\vec{r}_{2}$ are collinear; the singularity that happens at least when the angle between the two vectors is $\pi$ can be eliminated (PHILLIPS; SNYDER, 2000):

$$
\begin{gathered}
\vec{r}_{1} \cdot \vec{r}_{2}=r_{1} r_{2} \cos \theta \\
\left|\vec{r}_{1} \times \vec{r}_{2}\right|=r_{1} r_{2} \sin \theta \\
\frac{\left(\vec{r}_{2}-\vec{r}_{1}\right)}{\left|\vec{r}_{1} \times \vec{r}_{2}\right|^{2}} \cdot\left(\frac{\vec{r}_{1}}{r_{1}}-\frac{\vec{r}_{2}}{r_{2}}\right)=\frac{r_{1}+r_{2}}{r_{1} r_{2}\left(r_{1} r_{2}+\vec{r}_{1} \cdot \vec{r}_{2}\right)}
\end{gathered}
$$

Finally, the convenient equation (2.1) is obtained:

$$
\vec{V}_{V S}=\frac{\Gamma}{4 \pi} \frac{\left(r_{1}+r_{2}\right)\left(\vec{r}_{1} \times \vec{r}_{2}\right)}{r_{1} r_{2}\left(r_{1} r_{2}+\vec{r}_{1} \cdot \vec{r}_{2}\right)}
$$

\section{A.3 Resultant section potential force}

According to the KJT in its 3-D form, the force acting on the BC ' $\mathrm{j}$ ' of the lifting line is:

$$
\delta \vec{F}_{i}=\rho \vec{V}_{i} \times \vec{\Gamma}_{i} \delta l_{i}
$$

However, since $\vec{\Gamma}_{i}$ is parallel to the straight segment, it can be written in terms of its magnitude and the local span unit vector $\hat{s}_{i}$.

$$
\vec{\Gamma}_{i}=\Gamma_{i} \hat{s}_{i}
$$

Thus, the quantity $\vec{\Gamma}_{i} \delta l_{i}$ can be rewritten in a more convenient way for solving the system of equations through matrix inversion:

$$
\vec{\Gamma}_{i} \delta l_{i}=\Gamma_{i} \hat{s}_{i} \delta l_{i}=\Gamma_{i} \delta \vec{l}_{i}
$$

Substitution of this relation on equation (A.10) leads to equation (2.1.1):

$$
\delta \vec{F}_{i}=\rho \Gamma_{i} \vec{V}_{i} \times \delta \vec{l}_{i}
$$




\section{A.4 Representation of the resultant force vector in the wing coor- dinate system}

A wing section along with its local and global coordinate system is represented on figure $79 ; \hat{c}, \hat{n}$ and $\hat{s}$ are the unit vectors in the chordwise, normal, and spanwise directions, respectively and, at the same time, the axis of the local coordinate system. $\hat{X}, \hat{Z}$ and $\hat{Y}$ are the global coordinate system unit vectors: $\hat{X}$ is in the flow direction, $\hat{Z}$ in the normal direction, and $\hat{Y}$ into the global spanwise direction.

Figure 79 - 2-D force vectors representation along with local (airfoil section) and global (wing) reference frames.

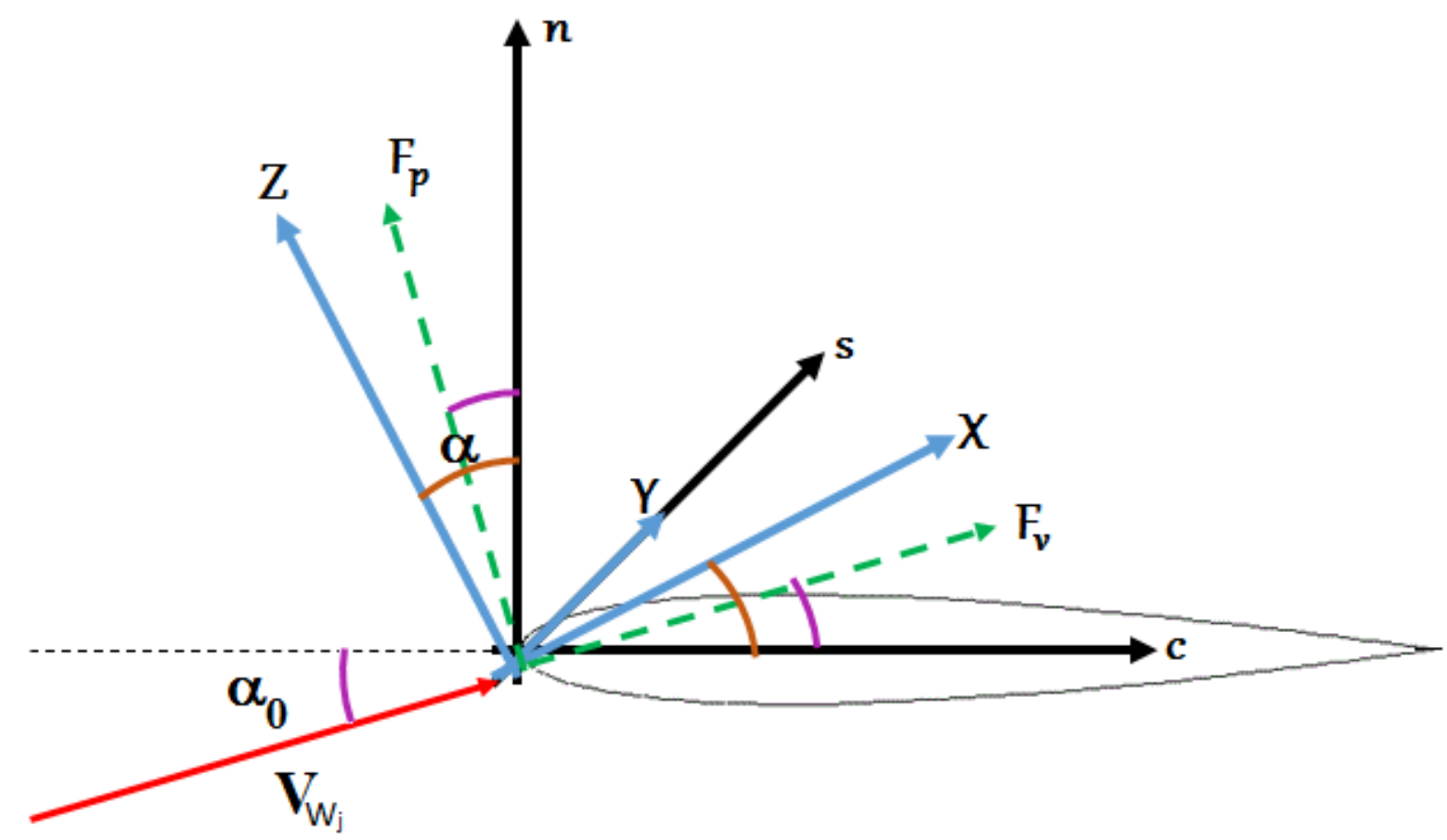

Source:Author

After convergence is attained, the resultant forces at this section are calculated with the aid of equation (2.12) and its respective $C_{c}$. These forces, however, are represented in the local coordinate system, and they must be represented in the global one for the calculations of the wing lift coefficients. With the assumption that hats and $\hat{Y}$ are parallel along the entire wingspan (i.e., there is no wing dihedral and the sections are as on figure 14 , the rotation matrix can be written with the aid of figure 79 :

$$
\left[\begin{array}{c}
\Delta D_{j} \\
0 \\
\Delta L_{j}
\end{array}\right]_{X Y Z}=\left[\begin{array}{ccc}
\cos \left(\alpha-\alpha_{0_{j}}\right) & 0 & \sin \left(\alpha-\alpha_{0_{j}}\right) \\
0 & 1 & 0 \\
-\sin \left(\alpha-\alpha_{0_{j}}\right) & 0 & \cos \left(\alpha-\alpha_{0_{j}}\right)
\end{array}\right] \times\left[\begin{array}{c}
\Delta F_{v_{j}} \\
0 \\
\Delta F_{p_{j}}
\end{array}\right]_{c s n}
$$


in which $\Delta F_{p_{j}}=\frac{1}{2} \rho_{\infty} V_{W_{j}}^{2} d A_{j} C_{n_{j}}$ and $\Delta F_{v_{j}}=\frac{1}{2} \rho_{\infty} V_{W_{j}}^{2} d A_{j} C_{c_{j}}$. The overall wing lift and drag forces are given by the the sum of the section forces:

$$
\begin{gathered}
D=\sum_{j=1}^{N} \Delta D_{j}=\frac{1}{2} \rho_{\infty} \sum_{j=1}^{N} V_{W_{j}}^{2} d A_{j}\left[C_{c_{j}} \cos \left(\alpha-\alpha_{0_{j}}\right)+C_{n_{j}} \sin \left(\alpha-\alpha_{0_{j}}\right)\right] \\
L=\sum_{j=1}^{N} \Delta L_{j}=\frac{1}{2} \rho_{\infty} \sum_{j=1}^{N} V_{W_{j}}^{2} d A_{j}\left[-C_{c_{j}} \sin \left(\alpha-\alpha_{0_{j}}\right)+C_{n_{j}} \cos \left(\alpha-\alpha_{0_{j}}\right)\right]
\end{gathered}
$$

When they are divided by $\frac{1}{2} \rho_{\infty} V_{\infty}^{2} S_{r}$, they become the overall wing lift and drag coefficients:

$$
\begin{aligned}
C_{L} & =\frac{L}{\frac{1}{2} \rho_{\infty} V_{\infty}^{2} S_{r}} \\
C_{D} & =\frac{D}{\frac{1}{2} \rho_{\infty} V_{\infty}^{2} S_{r}}
\end{aligned}
$$

$C_{n_{j}}$ and $C_{c_{j}}$ are, in fact, $C_{l}$ and $C_{d_{0}}$ in local coordinates. Due to the fact that they are not aligned with the global coordinates, they are then called $C_{n}$ and $C_{c}$. 